\title{
Computations and Measurements of the Effects of Trailing-Edge Geometry on the Wake of Bluff Bodies
}

\author{
by \\ Utku Caylan
}

A thesis submitted to the Faculty of Graduate and Postdoctoral Affairs in partial fulfillment of the requirements for the degree of

\author{
Master of Applied Science \\ in \\ Aerospace Engineering
}

\author{
Carleton University \\ Ottawa, Ontario
}

\author{
(C) 2019 \\ Utku Caylan
}


This thesis is dedicated to

my beloved parents and siblings

for their endless love, support, and encouragement. 


\begin{abstract}
This study has aimed to shed further light on the wake dynamics of a bluff body with a streamlined fore body and a rectangular blunt base through experiments and numerical analysis. Specifically, the study was designed to establish the role of instability modes and small-scale turbulence in shaping the wake dynamics, and the effect of boat tails including lobed geometries on the wake structure. In the absence of small-scale turbulence, the near wake of the body is dominated by the effects of two instability modes that promote larger-scale transient motions. Small-scale turbulence is found to have a significant suppressing effect on these motions. Presence of a straight tail is observed not to alter the fundamental structure of the wake, but the relative roles of the instability modes affecting the wake and the resultant streamwise mixing rates are modified. The straight tail is noted to reduce the initial size of the wake significantly to yield substantial reduction in form drag. Shaping the tail into a lobed trailing edge is found to modify the initial cross-section of the wake to an extent to negate the drag-reduction benefits produced by the tail in the straight configuration. Furthermore, the lobed configuration introduces three-dimensional coherent vortical structures at the tail trailing edge that dominate the near-wake development as well as promoting cross-stream mixing farther downstream.
\end{abstract}




\section{Acknowledgements}

First and foremost, I would like to thank my thesis supervisor, Dr. Metin I. Yaras, for his instruction, patience, advice, and encouragement. His intelligence and enthusiasm have undoubtedly shaped me for the better, into what I am today. I also wish to gratefully acknowledge Dr. Brian R. McAuliffe of the National Research Council of Canada (NRC) for his collaboration in the work.

Further, I would like to thank my close friend and colleague in our research group, Chanon Pretorius. Chanon and I started the journey of our masters at the same time and learnt a lot from each other over the course of our graduate years. It has been a blast working with him.

I am grateful to my fellow students and colleagues for not only their assistance, but also their friendship; these include (names are in alphabetical order) Adnan Poshtkouhian Badi, Andrei Bronipolsky, Brendan Ooi Xin-Zhi, Burak Oztaner, Chris Bassindale, Curtis Meister, John O'Keefe, Justin Kernot, Mark Kotwicz Herniczek, Omer Kaya, Salman Shafi, Shuhao Wu, Ted Gan, Wael Khaddage, Zachary Copeland, and many others. I appreciate Neil McFadyen for his technical assistance in maintaining and ensuring the proper operation of the computing resources of our research group. A special thanks to Sylvain Pitre and Ryan Taylor of the Carleton Research Computing Services for providing additional computing resources for this work. Their workshops on parallel computing and remote systems were very informative, and helped me better utilize our computing resources. I would also like to express my appreciation to Stephan Biljan for his diligence during the 3D printing process of the experimental model and its tails.

Most importantly, I would like to thank my beloved parents and siblings for their endless love, support, and encouragement.

Finally, the financial support of the National Research Council of Canada for this study is gratefully acknowledged. 


\section{Table of Contents}

Abstract $\quad$ iii

Acknowledgements $\quad$ iv

Table of Contents $\quad$ v

List of Tables viii

List of Figures $\quad$ ix

$\begin{array}{ll}\text { Nomenclature } & \text { Xv }\end{array}$

$\begin{array}{lll}\text { Chapter } 1 & \text { Introduction } & 1\end{array}$

$\begin{array}{lll}\text { Chapter } 2 & \text { Background } & 3\end{array}$

$\begin{array}{lll}\text { Chapter } 3 & \text { Literature Review } & 6\end{array}$

$\begin{array}{lll}\text { Chapter } 4 & \text { Experimental Setup } & 18\end{array}$

4.1 Geometry of Bluff-Body Model and Tails . . . . . . . . . . . . . . . . 18

4.2 Wind Tunnel Setup . . . . . . . . . . . . . . . . . . . 20

4.3 Operating Conditions . . . . . . . . . . . . . . . . 23

4.4 Instrumentation and Data Acquisition . . . . . . . . . . . 23

Chapter 5 Computational Models and Computational Method 24

5.1 Computational Models . . . . . . . . . . . . . . . . . 24

5.1.1 Computational Domain and Boundary Conditions . . . . . . . 24

5.1 .2 Computational Grid . . . . . . . . . . . . . 25

5.2 Computational Method . . . . . . . . . . . . . 28

5.2.1 Governing Equations and Turbulence Modeling . . . . . . . . 28

5.2.2 Discretization .................... 31 
5.2.3 Solution Method . . . . . . . . . . . . . . . . . . 32

Chapter $6 \quad$ Results and Discussion 33

6.1 Experimental Results . . . . . . . . . . . . . . . . 33

6.2 Computational Results . . . . . . . . . . . . . . . . 35

6.2.1 Baseline Configuration . . . . . . . . . . . . . . . 35

6.2.2 Straight-Tail Configuration . . . . . . . . . . . . . . 43

6.2.3 Lobed-Tail Configuration . . . . . . . . . . . . . . . . 47

Chapter $7 \quad$ Conclusions and Recommendations for Future Work 52

$\begin{array}{ll}\text { References } & 55\end{array}$

$\begin{array}{lll}\text { Appendix A } & \text { Wind-Tunnel Calibration } & 68\end{array}$

Appendix B Prediction of the Wake Dynamics for the Baseline Configuration Using OpenFOAM $\quad 69$

B.1 Computational Method . . . . . . . . . . . . . . 69

B.1.1 Discretization ................... 69

B.1.2 Solution Method . . . . . . . . . . . . . . . . 70

Appendix C Supplementary Plots on the Computational Results $\quad 73$

Appendix D Influence of Compressibility on Lobed-Mixer Performance 87

Appendix E Technical Drawings of the Bluff-Body Model and Tails 90

Bluff-Body Model \& Boat-Tail Assembly . . . . . . . . . . . . . . . . 90

Bluff-Body Model \& Boat-Tail Assembly: Exploded View . . . . . . . . . . 92

Bluff-Body Model . . . . . . . . . . . . . . . . . . . 93

Bluff-Body Model: Auxiliary Views . . . . . . . . . . . . . . . . . . 94

Bluff-Body Model: Contour Geometry . . . . . . . . . . . . . . . 95

Back Plate. . . . . . . . . . . . . . . . . . . 96

Boat-Tail ........................ . . . 97 
Straight Boat-Tail Extension . . . . . . . . . . . . . . . . . . 98

Curved Boat-Tail Extension . . . . . . . . . . . . . . . . . . . 99

Bluff-Body Model \& Lobed-Tail Assembly . . . . . . . . . . . . . . . . 100

Lobed-Tail . . . . . . . . . . . . . . . . . . . . . . . . . . . . . . 101

Small-Lobed Boat-Tail Extension _ . . . . . . . . . . . . . . . . . 102

$\begin{array}{lll}\text { Appendix F } & \text { Structural Analysis of Bluff-Body Model } & 103\end{array}$

F.1 Computational Model . . . . . . . . . . . . . . . . . . . . . . . 103

F.1.1 Computational Domain and Boundary Conditions . . . . . . 103

F.1.2 Spatial Grid . . . . . . . . . . . . . . . . . . . . . . 104

F.2 Computational Method . . . . . . . . . . . . . . . . 105

F.2.1 Governing Equations . . . . . . . . . . . . . . . 105

F.2.2 Discretization and Solution Method . . . . . . . . . . . 106

F.3 Results and Discussion . . . . . . . . . . . . . . . . . 106 


\section{List of Tables}

4.1 Geometric parameters of the bluff-body model . . . . . . . 18

4.2 Geometric parameters of the tails . . . . . . . . . . . 19

5.1 Grid specifications . . . . . . . . . . . . . 28

5.2 Closure constants in the SST turbulence model . . . . . . . . 30

6.1 Measured drag coefficients . . . . . . . . . . . . . 33

$6.2 \quad$ Comparison of $C_{D}$ obtained from the simulations . . . . . 51

F.1 Grid specifications . . . . . . . . . . . . 105 


\section{List of Figures}

1.1 Schematic of a generic transport truck with boat-tail and other drag-reduction devices . . . . . . . . . . . . . 2

2.1 Schematics of streamlines in the vicinity of a) an airfoil at low angle of attack, b) an airfoil at high angle of attack, and c) a circular disk . . . . . . . . . . . . . . . . . .

2.2 Schematic of the wake development for a 2D cylinder in crossflow (adapted from Taneda [7]) . . . . . . . . . . . 4

3.1 Dominant flow structures in the wake of sphere at $\mathbf{R e}_{\mathbf{D}}=\mathbf{1 0 0 0}$ (adapted from Achenbach [38]) . . . . . . . . . . 7

3.2 Instability modes of A and B in the wake of a 2D cylinder . . 10

3.3 Wake development behind the Ahmed body in ground proximity 11

3.4 Streamline patterns illustrating the wake bistability of a squareback Ahmed body in ground proximity at $\mathbf{R e}_{\mathbf{H}} \approx \mathbf{1 0}^{\mathbf{5}}[89]$. . 12

3.5 Schematic of a generic lobed mixer . . . . . . . . . . . . 14

3.6 Schematic of the vortical structures downstream of the lobedmixer (adapted from McCormick and Bennett [122]) . . . . . 16

4.1 Schematic of the bluff-body model . . . . . . . . . . . 18

4.2 Schematics of a) straight tail b) curved tail and c) lobed tail . 19

4.3 Schematic of wind-tunnel facility . . . . . . . . . . . . 20

4.4 Cross-sectional view of the slotted-wall test section . . . . . 21

4.5 Schematic of model installation in the test section . . . . . . 22

$5.1 \quad$ Schematic of the computational domain . . . . . . . . . . 24

$5.2 \quad$ Spatial grid for the baseline case . . . . . . . . . . . 26

$5.3 \quad$ Spatial grid for the straight-tail case . . . . . . . . . 27

$5.4 \quad$ Spatial grid for the lobed-tail case . . . . . . . . . . . . 27

5.5 Grid-cell nomenclature . . . . . . . . . . . . 28 
$6.1 \quad$ Experimental tail configurations . . . . . . . . . . 33

$6.2 \mathrm{Q} /\left(\mathbf{U}_{\text {ref }} / \mathbf{D}_{\mathbf{h}}\right)^{2}=\mathbf{9 . 5}$ isosurfaces for the baseline configuration in the absence of small-scale turbulence . . . . . . . . . . . .

$6.3 \mathrm{Q} /\left(\mathbf{U}_{\text {ref }} / \mathbf{D}_{\mathbf{h}}\right)^{2}=\mathbf{9 . 5}$ isosurfaces in the near wake for the baseline configuration in the absence of small-scale turbulence . . .

6.4 Schematic representation of the wake structure for a) the sphere [43], and b) the present bluff body . . . . . . . . . . . 38

$6.5 \mathrm{Q} /\left(\mathbf{U}_{\text {ref }} / \mathbf{D}_{\mathrm{h}}\right)^{2}=1.2 \times 10^{-3}$ isosurfaces for the baseline configuration in the presence of small-scale turbulence . . . . . . .

6.6 Schematic representation of the wake structure a) in the absence of small-scale turbulence, b) in the presence of small-scale

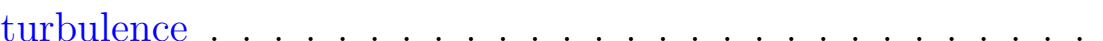

6.7 Planes of interest in the bluff-body wake . . . . . . . 40

6.8 Kinetic energy of larger-scale transient structures in the absence of small-scale turbulence for the baseline configuration . . . . 41

6.9 Length scale of larger-scale transient structures for the baseline configuration in the absence of small-scale turbulence . . . . . 41

6.10 Axial momentum flux distribution in the wake for the baseline configuration in the absence of small-scale turbulence . . . . . 42

6.11 Axial momentum flux distribution in the wake for the baseline configuration in the presence of small-scale turbulence . . . . .

$6.12 \mathrm{Q} /\left(\mathbf{U}_{\text {ref }} / \mathbf{D}_{\mathrm{h}}\right)^{2}=\mathbf{9 . 5}$ isosurfaces for the straight-tail configuration in the absence of small-scale turbulence . . . . . . . . . 43

$6.13 \mathrm{Q} /\left(\mathrm{U}_{\text {ref }} / \mathrm{D}_{\mathrm{h}}\right)^{2}=\mathbf{9 . 5}$ isosurfaces in the near wake for the straighttail configuration in the absence of small-scale turbulence . . . 44

$6.14 \mathrm{Q} /\left(\mathrm{U}_{\mathrm{ref}} / \mathrm{D}_{\mathrm{h}}\right)^{2}=\mathbf{2 . 4} \times \mathbf{1 0}^{-\mathbf{2}}$ isosurfaces for the straight-tail configuration in the presence of small-scale turbulence . . . .

6.15 Kinetic energy of larger-scale transient structures in the absence of small-scale turbulence for the straight-tail configuration . . 45 
6.16 Length scale of larger-scale transient structures for the straighttail configuration in the absence of small-scale turbulence . . . 46

6.17 Axial momentum flux distribution in the wake for the straighttail configuration in the absence of small-scale turbulence . . . 46

6.18 Axial momentum flux distribution in the wake for the straighttail configuration in the presence of small-scale turbulence . . 47

$6.19 \mathrm{Q} /\left(\mathbf{U}_{\text {ref }} / \mathbf{D}_{\mathbf{h}}\right)^{\mathbf{2}}=\mathbf{9 . 5}$ isosurfaces for the lobed-tail configuration in the absence of small-scale turbulence . . . . . . . . 47

$6.20 \mathrm{Q} /\left(\mathbf{U}_{\text {ref }} / \mathbf{D}_{\mathbf{h}}\right)^{2}=\mathbf{9 . 5}$ isosurfaces in the near wake for the lobedtail configuration in the absence of small-scale turbulence . . . 48

$6.21 \mathrm{Q} /\left(\mathbf{U}_{\text {ref }} / \mathbf{D}_{\mathbf{h}}\right)^{2}=\mathbf{2 . 4} \times \mathbf{1 0}^{-2}$ isosurfaces for the lobed-tail configuration in the presence of small-scale turbulence . . . . . . 49

6.22 Axial momentum flux distribution in the wake for the lobed-tail configuration in the absence of small-scale turbulence . . . . .

6.23 Kinetic energy of larger-scale transient structures in the absence of small-scale turbulence for the lobed-tail configuration . . . . 50

6.24 Length scale of larger-scale transient structures for the lobedtail configuration in the absence of small-scale turbulence . . . 50

A.1 Schematic of the wind-tunnel measurement stations . . . . 68

A.2 Correlation of test-section inlet dynamic pressure to the contraction pressure difference . . . . . . . . . . . . 68

B.1 $\mathbf{Q} /\left(\mathbf{U}_{\text {ref }} / \mathbf{D}_{\mathbf{h}}\right)^{2}=\mathbf{9 . 5}$ isosurfaces in a) OpenFOAM, b) CFX for the baseline configuration in the absence of small-scale turbulence 72

C.1 $\mathrm{Q} /\left(\mathbf{U}_{\text {ref }} / \mathbf{D}_{\mathrm{h}}\right)^{2}=\mathbf{9 . 5}$ isosurfaces in the baseline configuration in the absence of small-scale turbulence . . . . . . . . . . 73

C.2 Streamtrace ribbons with transculent isosurfaces of $\mathbf{Q} /\left(\mathbf{U}_{\text {ref }} / \mathbf{D}_{\mathbf{h}}\right)^{2}=$ 9.5 in the absence of small-scale turbulence in the baseline con-

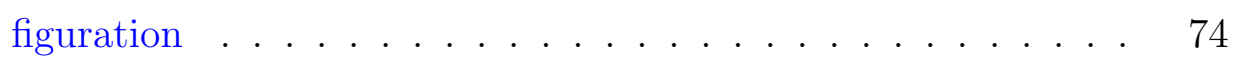


C.3 Streamtrace ribbons with transculent isosurfaces of $\mathbf{Q} /\left(\mathbf{U}_{\text {ref }} / \mathbf{D}_{\mathbf{h}}\right)^{2}=$ $\mathbf{9 . 5}$ in the absence of small-scale turbulence in the straight-tail configuration ..................... 74

C.4 Streamtrace ribbons with transculent isosurfaces of $\mathbf{Q} /\left(\mathbf{U}_{\text {ref }} / \mathbf{D}_{\mathbf{h}}\right)^{2}=$ 9.5 in the absence of small-scale turbulence in the lobed-tail configuration ..................... 75

C.5 Streamtrace ribbons with transculent isosurfaces of $\mathbf{Q} /\left(\mathbf{U}_{\text {ref }} / \mathbf{D}_{\mathbf{h}}\right)^{2}=$ $\mathbf{1 . 2} \times \mathbf{1 0}^{-\mathbf{3}}$ in the presence of small-scale turbulence in the baseline configuration . . . . . . . . . . . . . 75

C.6 Streamtrace ribbons with transculent isosurfaces of $\mathbf{Q} /\left(\mathbf{U}_{\text {ref }} / \mathbf{D}_{\mathbf{h}}\right)^{2}=$ $\mathbf{1 . 2 5} \times \mathbf{1 0}^{-\mathbf{2}}$ in the presence of small-scale turbulence in the straight-tail configuration ............ . . 76

C.7 Streamtrace ribbons with transculent isosurfaces of $\mathbf{Q} /\left(\mathbf{U}_{\text {ref }} / \mathbf{D}_{\mathbf{h}}\right)^{2}=$ $2.4 \times 10^{-2}$ in the presence of small-scale turbulence in the lobed-tail configuration . . . . . . . . . . . . 76

C.8 $\mathrm{Q} /\left(\mathrm{U}_{\text {ref }} / \mathrm{D}_{\mathrm{h}}\right)^{\mathbf{2}}=\mathbf{2 . 4} \times \mathbf{1 0}^{-\mathbf{1}}$ isosurfaces calculated based on sequentially time-averaged flow fields with an averaging period of $10 \mathrm{~K}-\mathrm{H}$ cycles in the baseline configuration in the absence of small-scale turbulence . . . . . . . . . . . . . . 77

C.9 $\mathrm{Q} /\left(\mathrm{U}_{\text {ref }} / \mathbf{D}_{\mathbf{h}}\right)^{\mathbf{2}}=\mathbf{2 . 4} \times \mathbf{1 0}^{-1}$ isosurfaces calculated based on sequentially time-averaged flow fields with an averaging period of $10 \mathrm{~K}-\mathrm{H}$ cycles in the straight-tail configuration in the absence of small-scale turbulence . . . . . . . . . . . . . 78

C.10 Q $/\left(\mathbf{U}_{\text {ref }} / \mathbf{D}_{\mathbf{h}}\right)^{2}=\mathbf{2 . 4} \times \mathbf{1 0}^{-1}$ isosurfaces calculated based on sequentially time-averaged flow fields with an averaging period of $10 \mathrm{~K}-\mathrm{H}$ cycles in the lobed-tail configuration in the absence of small-scale turbulence . . . . . . . . . . . . . . . 
C.11 $\mathrm{Q} /\left(\mathrm{U}_{\text {ref }} / \mathrm{D}_{\mathbf{h}}\right)^{2}=\mathbf{2 . 4} \times \mathbf{1 0}^{-1}$ isosurfaces calculated based on sequentially time-averaged flow fields with an averaging period of $10 \mathrm{~K}-\mathrm{H}$ cycles in the baseline configuration in the presence of small-scale turbulence . . . . . . . . . . . . . . .

C.12 Q $/\left(\mathrm{U}_{\text {ref }} / \mathrm{D}_{\mathbf{h}}\right)^{2}=\mathbf{2 . 4} \times \mathbf{1 0}^{-\mathbf{1}}$ isosurfaces calculated based on sequentially time-averaged flow fields with an averaging period of $10 \mathrm{~K}-\mathrm{H}$ cycles in the straight-tail configuration in the presence of small-scale turbulence . . . . . . . . . . . . . . . .

C.13 Q $/\left(\mathrm{U}_{\text {ref }} / \mathrm{D}_{\mathrm{h}}\right)^{2}=\mathbf{2 . 4} \times \mathbf{1 0}^{-1}$ isosurfaces calculated based on sequentially time-averaged flow fields with an averaging period of $10 \mathrm{~K}-\mathrm{H}$ cycles in the lobed-tail configuration in the presence of small-scale turbulence . . . . . . . . . . . . . . .

C.14 Q $/\left(\mathrm{U}_{\text {ref }} / \mathrm{D}_{\mathrm{h}}\right)^{2}=2.4 \times \mathbf{1 0}^{-1}$ isosurfaces calculated based on sequentially time-averaged flow fields with an averaging period of $25 \mathrm{~K}-\mathrm{H}$ cycles in the baseline configuration in the absence of small-scale turbulence . . . . . . . . . . . . . .

C.15 Q $/\left(\mathrm{U}_{\text {ref }} / \mathrm{D}_{\mathrm{h}}\right)^{2}=\mathbf{2 . 4} \times \mathbf{1 0}^{-1}$ isosurfaces calculated based on sequentially time-averaged flow fields with an averaging period of $25 \mathrm{~K}-\mathrm{H}$ cycles in the straight-tail configuration in the absence of small-scale turbulence . . . . . . . . . . . . . . .

C.16 Q $/\left(\mathrm{U}_{\text {ref }} / \mathrm{D}_{\mathrm{h}}\right)^{2}=\mathbf{2 . 4} \times \mathbf{1 0}^{-1}$ isosurfaces calculated based on sequentially time-averaged flow fields with an averaging period of $25 \mathrm{~K}-\mathrm{H}$ cycles in the lobed-tail configuration in the absence of small-scale turbulence . . . . . . . . . . . . . . .

C.17 Pressure variation along the wake centerline a) in the absence of small-scale turbulence, b) in the presence of small-scale turbulence 86

C.18 Pressure variation along the bluff-body surface in the x-z plane a) in the absence of small-scale turbulence, b) in the presence of small-scale turbulence . . . . . . . . . . . . . . 86 
F.1 Schematic of model installation and mounting tabs . . . . 103

F.2 Schematic of computational domain and boundary conditions 104

F.3 $\sigma_{\mathbf{e}} / \sigma_{\text {nom }}$ contours at the a) bottom, b) top of the plate $\ldots 107$ 


\section{Nomenclature}

$a \quad$ fillet major-axis length (m) (Fig. 4.1); speed of sound (m/s) (Eq. D.1)

$a_{1} \quad$ modeling constant (Eq. 5.3)

$A \quad$ cross-sectional area $\left(\mathrm{m}^{2}\right)$ (Fig. 4.1); sparse coefficient matrix

AR model aspect ratio (Fig. 4.1)

$b \quad$ fillet minor-axis length (m) (Fig. 4.1); forcing vector of source terms

C Courant number

$C_{D} \quad$ aerodynamic drag coefficient (Eq. 4.1)

$C_{P} \quad$ pressure coefficient (Fig. C.17)

$C_{\mu} \quad$ modeling constant (Eq. 5.4)

$d \quad$ lobe width $(\mathrm{m})$ (Fig. 4.2)

$D \quad$ aerodynamic drag force (N) (Eq. 4.1); cylinder/sphere diameter (m)

$D_{\text {fan }}$ wind-tunnel fan diameter (m) (Fig. 4.3)

$D_{h} \quad$ model hydraulic diameter (m) (Fig. 4.1)

E Young's modulus $\left(\mathrm{N} / \mathrm{m}^{2}\right)$ (Fig. F.2)

$f \quad$ vortex shedding frequency $(\mathrm{Hz})$; body force vector magnitude per unit volume $\left(\mathrm{N} / \mathrm{m}^{3}\right)($ Eq. F.2)

$F_{1} \quad$ blending function (Eq. 5.3)

$F_{2} \quad$ blending function (Eq. 5.6) 
$g \quad$ gravitational acceleration $\left(\mathrm{m} / \mathrm{s}^{2}\right)$ (Fig. F.2)

$h \quad$ lobe height (m) (Fig. 4.2)

$H \quad$ model height (m) (Fig. 4.1); tail height (m) (Fig. 4.2)

I turbulence intensity (Eq. 5.9)

$k \quad$ turbulence kinetic energy $\left(\mathrm{m}^{2} / \mathrm{s}^{2}\right)($ Eq. 5.9)

$k_{L S} \quad$ turbulence kinetic energy of large-scale coherent structures $\left(\mathrm{m}^{2} / \mathrm{s}^{2}\right)$ (Eq. 6.2)

L model length (m) (Fig. 4.1); tail length (m) (Fig. 4.2); turbulence integral length scale (m) (Eq. 5.10)

$L_{\max }$ maximum edge length of a grid cell (m) (Fig. 5.5)

$L_{\text {min }}$ minimum edge length of a grid cell (m) (Fig. 5.5)

$m \quad \operatorname{mass}(\mathrm{kg})$

$M_{c} \quad$ convective Mach number (Eq. D.4)

$p \quad$ static pressure $\left(\mathrm{N} / \mathrm{m}^{2}\right)$

$P_{k} \quad$ rate of production of turbulence kinetic energy (Eq. 5.7)

$q \quad$ dynamic pressure $\left(\mathrm{N} / \mathrm{m}^{2}\right)$ Fig. A.2

$Q \quad$ second invariant of velocity-gradient tensor $\left(1 / \mathrm{s}^{2}\right)$ (Eq. 6.1)

$r \quad$ radius of curvature $(\mathrm{m})$ (Fig. 4.2$)$

$R_{1} \quad$ circumradius of an actual grid cell (m) (Fig. 5.5)

$R_{2} \quad$ circumradius of an equilateral grid cell (m) (Fig. 5.5)

$R e_{c} \quad$ Reynolds mumber based on chord length, $R e_{c}=U c / \nu$

$R e_{D}$ Reynolds mumber based on cylinder/sphere diameter, $R e_{D}=U D / \nu$ 
$R e_{D_{h}}$ Reynolds mumber based on model hydraulic diameter, $R e_{D}=U D_{h} / \nu$

$R e_{H} \quad$ Reynolds mumber based on model height, $R e_{H}=U H / \nu$ (Fig. $3.3 \& 3.4$ )

$S_{t} \quad$ magnitude of local strain rate (Eq. 5.3)

$S t_{D} \quad$ Instability Strouhal number based on cylinder/sphere diameter, $S t_{D}=f D / U_{c}$

$S t_{D_{h}}$ Instability Strouhal number based on model hydraulic diameter, $S t_{D_{h}}=f D_{h} / U_{c}$

$S t_{\theta} \quad$ Instability Strouhal number based on momentum thickness, $S t_{\theta}=f \theta / U_{c}$

$t \quad$ time $(\mathrm{s})$

$u \quad$ nodal displacement (m) (Eq. F.3)

$u^{\prime} \quad$ velocity fluctuation $(\mathrm{m} / \mathrm{s})($ Eq. 6.2$)$

$u_{\tau} \quad$ friction velocity $(\mathrm{m} / \mathrm{s}), u_{\tau}=\sqrt{\tau_{w} / \rho}$

$U \quad$ free-stream velocity $(\mathrm{m} / \mathrm{s})$

$U_{c} \quad$ convective velocity $(\mathrm{m} / \mathrm{s})($ Eq. D.3)

$W \quad$ model width (m) (Fig. 4.1); tail width (m) (Fig. 4.2)

$x \quad$ streamwise spatial coordinate $(\mathrm{m})$

y wall-normal spatial coordinate $(\mathrm{m})$

$y_{1}^{+} \quad$ normalized distance for the first grid node off the wall

$z \quad$ spanwise spatial coordinate $(\mathrm{m})$

\section{Greek Letters}

$\alpha_{1} \quad$ modeling constant (Eq. 5.6)

$\alpha_{2} \quad$ modeling constant (Eq. 5.6) 
$\alpha_{\omega 1} \quad$ modeling constant (Eq. 5.6)

$\alpha_{\omega 2} \quad$ modeling constant (Eq. 5.6)

$\alpha_{k 1} \quad$ modeling constant (Eq. 5.5)

$\alpha_{k 2} \quad$ modeling constant (Eq. 5.6)

$\beta \quad$ obliqueness angle of velocity perturbation (deg.) (Eq. D.5)

$\beta_{1} \quad$ modeling constant (Eq. 5.6)

$\beta_{2} \quad$ modeling constant (Eq. 5.6)

$\Delta A$ normal area of each data point in planes of interest $\left(\mathrm{m}^{2}\right)$ (Fig. 6.7)

$\Delta t \quad$ time-step size (s)

$\Delta x \quad$ spacing of the spatial grid in numerical model $(\mathrm{m})$

$\delta_{i} \quad$ Kronecker delta

$\epsilon \quad$ turbulence dissipation rate $\left(\mathrm{m}^{2} / \mathrm{s}^{3}\right)($ Eq. 5.4)

$\epsilon_{1} \quad$ top/bottom-tail angle (deg.) (Fig. 4.2)

$\epsilon_{2} \quad$ side-tail angle (deg.) (Fig. 4.2)

$\epsilon_{c} \quad$ outer penetration angle (deg.) (Fig. 4.2)

$\epsilon_{t} \quad$ inner penetration angle (deg.) (Fig. 4.2)

$\gamma \quad$ specific heat ratio (Eq. D.2)

$\lambda \quad$ Lame's coefficient (Eq. F.4)

$\mu \quad$ dynamic viscosity (kg/(m.s)); Lame's coefficient (Eq. F.4)

$\nu \quad$ kinematic viscosity $\left(\mathrm{m}^{2} / \mathrm{s}\right)$ (Eq. 5.2); Poisson's ratio (Fig. F.2) 
$\nu_{t} \quad$ eddy viscosity $\left(\mathrm{m}^{2} / \mathrm{s}\right)(\mathrm{Eq} .5 .3)$

$\omega \quad$ specific turbulence dissipation rate (1/s) (Eq. 5.4)

$\omega_{\text {wall }}$ specific turbulence dissipation rate at the wall $(1 / \mathrm{s})($ Eq. 5.8)

$\bar{\phi} \quad$ time average of dependent vector variable $\phi$

$\rho \quad$ air density $\left(\mathrm{kg} / \mathrm{m}^{3}\right)($ Eq. 4.1$)$

$\rho_{A B S}$ density of acrylonitrile butadiene styrene plastic $\left(\mathrm{kg} / \mathrm{m}^{3}\right)$ (Fig. F.2)

$\sigma \quad$ compressive/tensile stress $\left(\mathrm{N} / \mathrm{m}^{2}\right)($ Fig. F.2)

$\sigma_{y} \quad$ yield stress $\left(\mathrm{N} / \mathrm{m}^{2}\right)($ Fig. F.2)

$\tau_{w} \quad$ local wall shear stress $\left(\mathrm{N} / \mathrm{m}^{2}\right)$

$\theta \quad$ shear-layer momentum thickness (m)

$\varepsilon \quad$ engineering strain (Eq. F.4)

\section{Subscripts}

1 high-speed stream quantity

$2 \quad$ low-speed stream quantity

c corner

$c_{e f f}$ effective convective

e von Mises equivalent

$i, j \quad$ indices for the $\mathrm{x}, \mathrm{y}$, and $\mathrm{z}$ coordinate directions

$J \quad$ wind-tunnel measurement station at the test-section inlet

$L \quad$ wind-tunnel measurement station immediately downstream of flow conditioners 


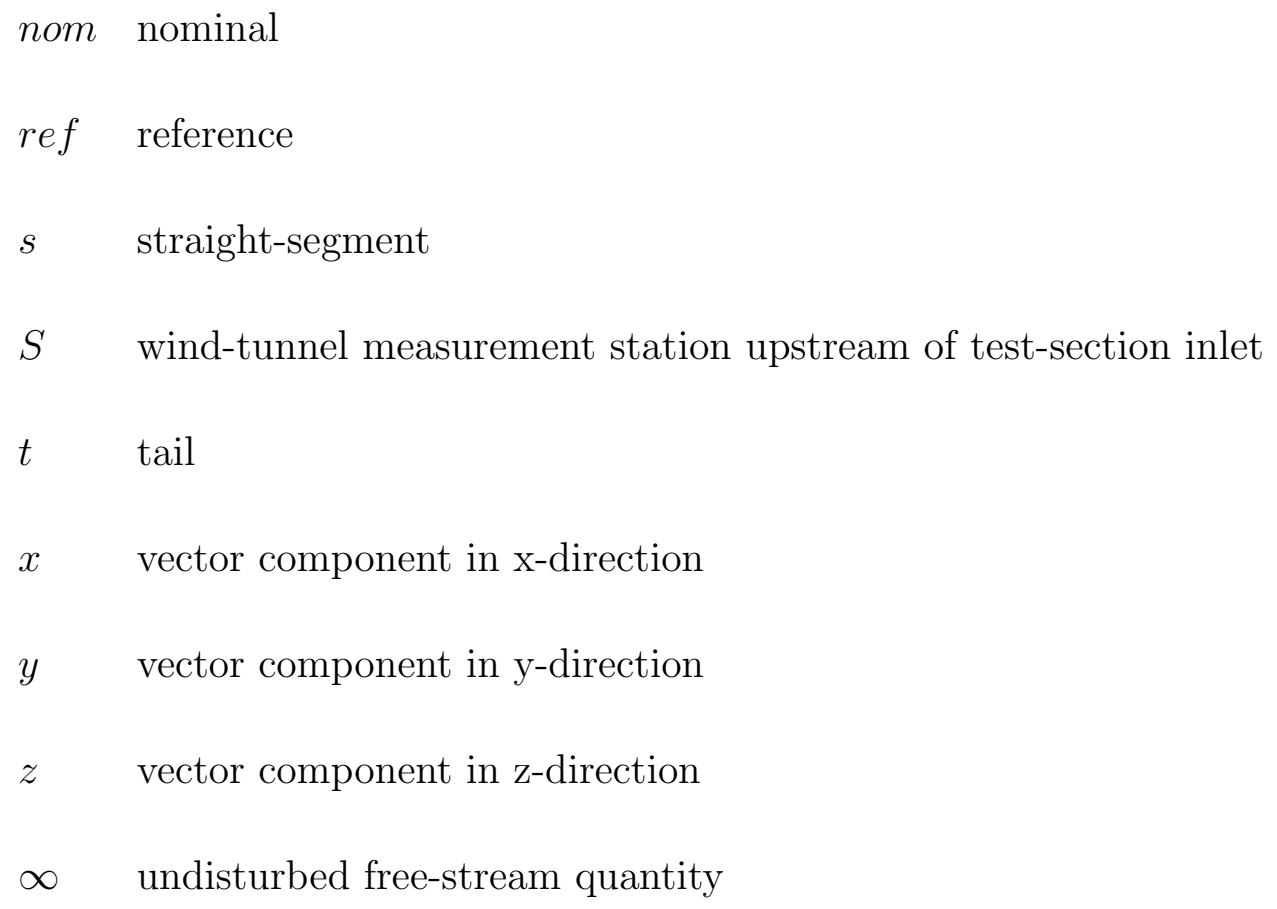




$\begin{array}{ll}\text { OpenFOAM } & \text { Open source Field Operation And Manipulation } \\ \text { PCG } & \text { preconditioned conjugate } \\ \text { PIMPLE } & \text { blended algorithm of PISO and SIMPLE } \\ \text { PISO } & \text { Pressure-Implicit-Split-Operator } \\ \text { RANS } & \text { Reynolds-averaged Navier-Stokes } \\ \text { RSB } & \text { reflectional symmetry breaking } \\ \text { SIMPLE } & \text { Semi-Implicit-Method-of-Pressure-Linked-Equations } \\ \text { SST } & \text { Shear-Stress-Transport }\end{array}$




\section{Chapter 1}

\section{Introduction}

Bluff bodies are encountered in many practical aerodynamic applications such as bridges, buildings, and ground transportation. Unlike their streamlined counterparts, bluff bodies experience large regions of flow separation. The presence of separated flow in the wake of bluff bodies results in relatively large magnitudes of form drag. Further, the aerodynamic forces associated with the large scale transient flow structures prevailing in the wake may be hazardous in certain applications. Active and passive control techniques to counter the adverse effects of the wake region has therefore attracted increasing engineering interest in recent decades.

One of the application areas that has been stimulating such research is the transport truck industry with the objectives of improved fuel efficiency, vehicle stability enhancement, wake hazard minimization, and aerodynamic noise reduction [1,2]. Majority of this research has been conducted on passive control techniques due to simplicity of their implementation. Devices appended to the rear of the trucks and their trailers have been found to be most promising in yielding reduction in aerodynamic drag [3]. One such device, referred to as a boat-tail, is shown on a generic transport truck in Figure 1.1. Since early 2000s, the California Air Resources Board (CARB) and the U.S. Environmental Protection Agency (EPA) have been encouraging the use of boat-tails with lengths up to $1.2 \mathrm{~m}$ (7.5\% of the length of a standard tractor-trailer) with a potential $5 \%$ reduction in fuel consumption [4]. In Canada, boat-tails were initially restricted to a length of 0.6 m. In 2010, Transport Canada's Road Safety and Motor Vehicle Regulation Directorate commissioned a preliminary study, partly performed by the National Research Council of Canada (NRC), towards a change in the Canadian Motor Vehicle Safety Regulations to allow longer boat-tails. In 2013, Canadian Motor Vehicle Safety Standard 223 was amended, allowing boat-tails to extend 


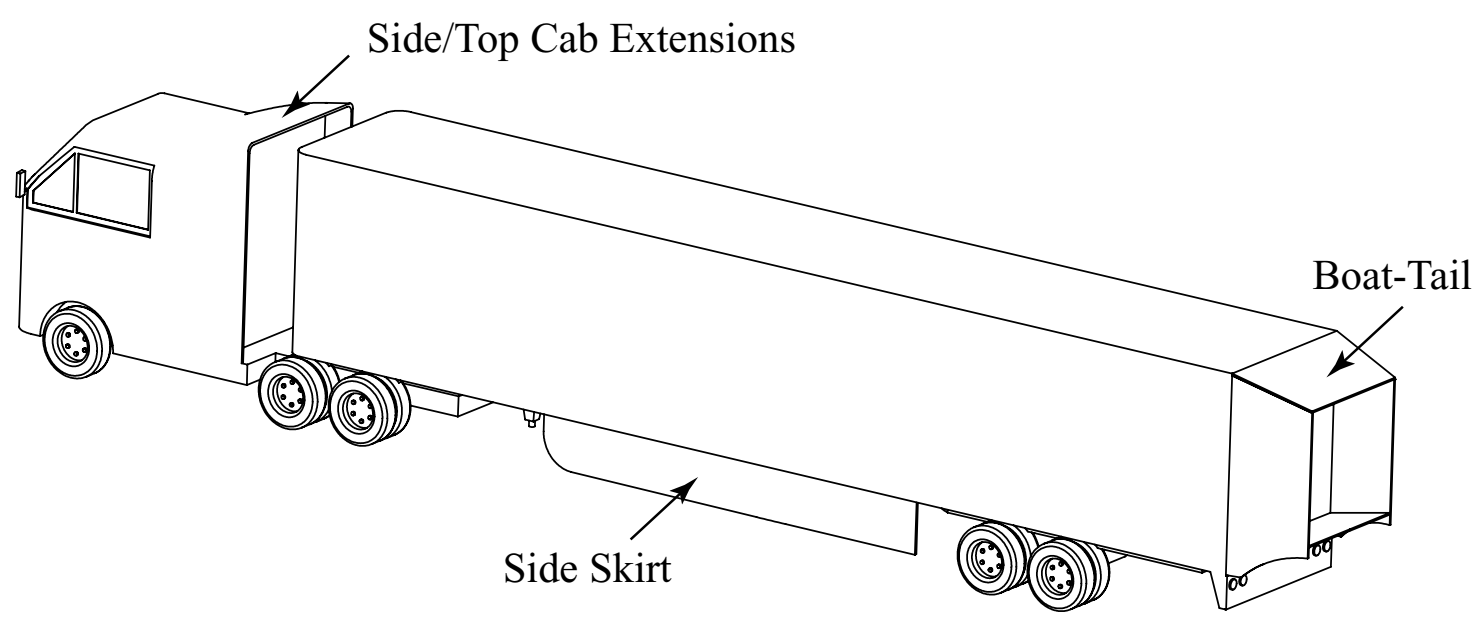

Figure 1.1: Schematic of a generic transport truck with boat-tail and other drag-reduction devices

up to $1.2 \mathrm{~m}$ [5]. More recently, boat-tails have been under investigation by the NRC with the purpose of aerodynamic performance optimization through Transport Canada's ecoTECHNOLOGY for Vehicles program [6].

Despite extensive research spanning several decades, the dynamics in the wake region of bluff bodies is still not sufficiently understood. This lack of understanding has hindered further optimization of passive flow control techniques for the wake region, such as the boat-tail. In the context of tractor trailers, the challenge stems, in part, from the mutually interacting aerodynamic effects of the geometric features of the vehicle spanning a broad range of length scales. A layered approach wherein the effects of individual geometric features on the prevailing aerodynamics are studied first in isolation and then in combinations of increasing complexity has often proven to be an effective strategy. This strategy was adopted in the present study by utilizing a bluff body shaped to produce a well-defined boundary layer at the trailing edge. The study is based on complementary use of wind-tunnel experiments and numerical solution of the governing Navier-Stokes equations in Reynolds-averaged form. 


\section{Chapter 2}

\section{Background}

This chapter is intended for readers unfamiliar with the basic flow physics of bluff-body wakes. Readers familiar with this subject may begin their reading with Chapter 3, which presents the state-of-the-art on the subject through a review of relevant literature.

Bodies placed in a fluid stream are classified into two categories: streamlined bodies, and bluff bodies. Figure 2.1 illustrates the streamline patterns in the vicinity of such bodies. Streamlined bodies keep the flow substantially attached to their

a)

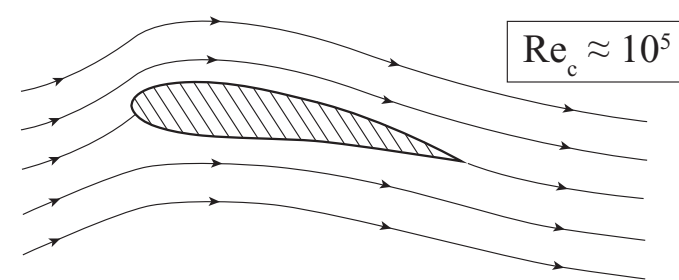

Form drag contribution

b)

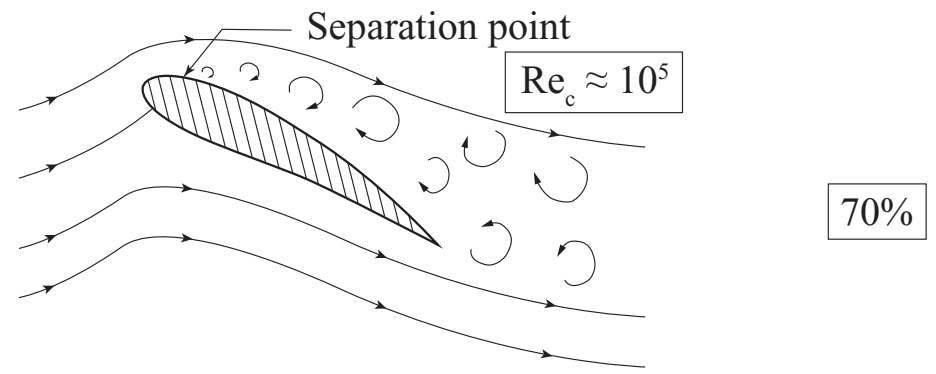
Separation point

c)

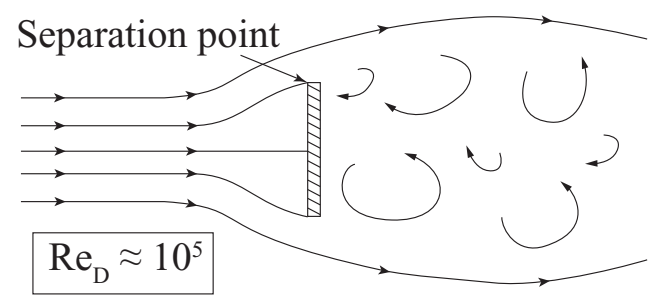

$70 \%$

$98 \%$

Figure 2.1: Schematics of streamlines in the vicinity of a) an airfoil at low angle of attack, b) an airfoil at high angle of attack, and c) a circular disk 
surface, whereas bluff bodies exhibit large regions of separation over a substantial proportion of their body surface that significantly alters the streamline pattern in the flow field of the body. The viscous drag force acting on a body immersed in a fluid stream consists of the skin-friction drag and form/pressure drag. The former is the result of the no-slip flow condition on the body surface prompting the development of a shear layer along the body surface, commonly referred to as the boundary layer. Form or pressure drag is the result of the difference in the pressures acting on the windward and leeward sides of the body.

As an example, Figure 2.2 illustrates the progression of the wake development with increased Reynolds number for a 2D (infinitely long) cylinder in crossflow. At low Reynolds numbers, the boundary layers and the wake of a bluff body remain stable. At a critical Reynolds number, one or more viscous and inviscid modes of flow

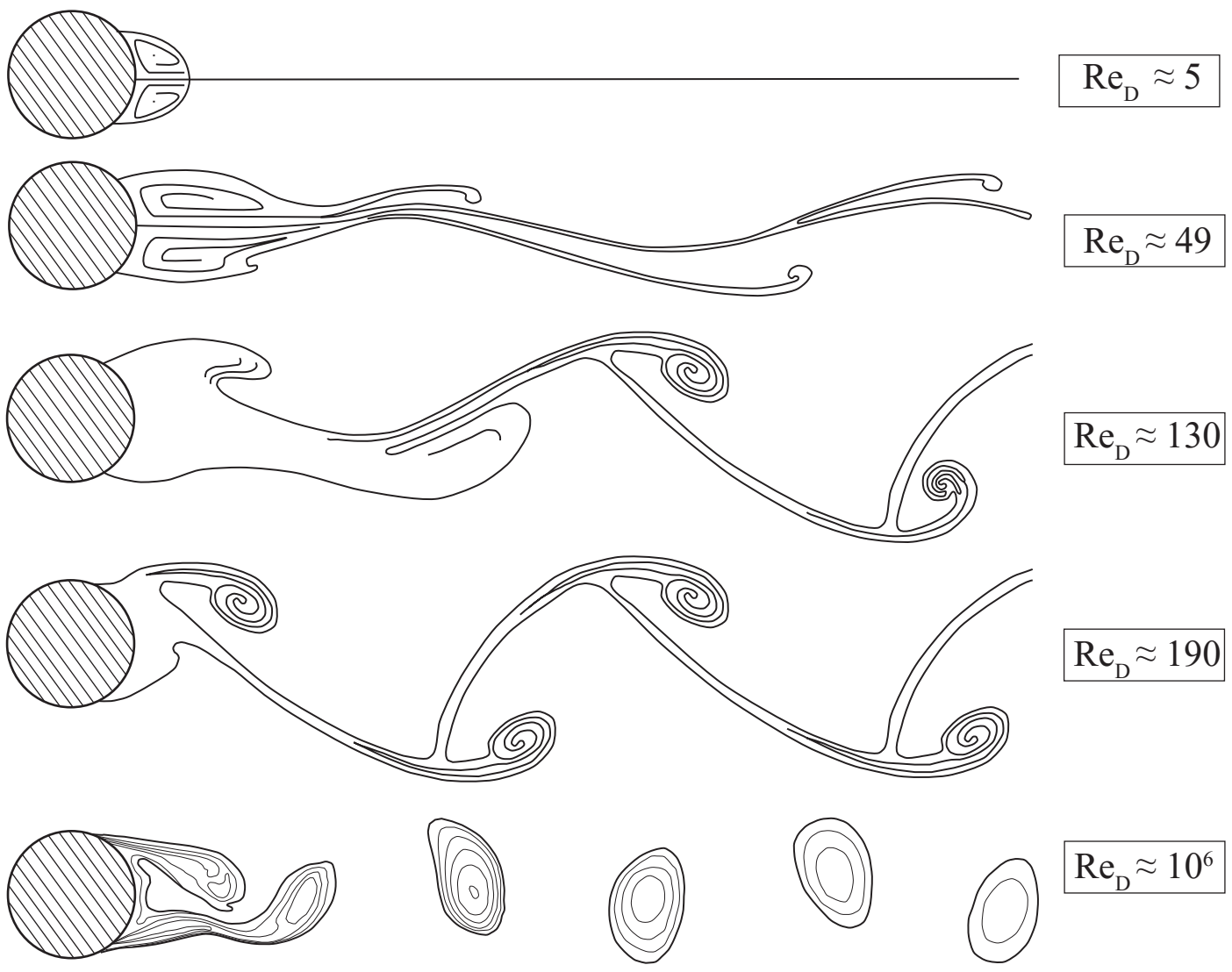

Figure 2.2: Schematic of the wake development for a $2 \mathrm{D}$ cylinder in crossflow (adapted from Taneda [7]) 
instabilities arise. Multiple modes of flow instabilities may co-exist. A characteristic feature of bluff-body wakes above the critical Reynolds number is the periodic vortex shedding for both laminar and turbulent flow states [8-10]. While periodic vortex shedding is often associated with relatively low values of flow Reynolds numbers, this does not necessarily imply that the instabilities responsible for this flow development cease to exist at higher flow Reynolds numbers. There is evidence to suggest that the inviscid instability mode driving periodic vortex shedding continues to be active up to Reynolds number values of $9^{\text {th }}$ order $[11,12]$. While both vortex shedding and wake turbulence promote pressure fluctuations on the bluff-body surface, vortex shedding is the primary source of the larger transient aerodynamic wake forces on the body $[13-15]$. 


\section{Chapter 3}

\section{Literature Review}

The wakes of three-dimensional bluff bodies is of considerable engineering interest as they relate to a wide range of applications, with the transportation industry being one example. Such wakes result in high pressure or form drag caused by flow separation and associated transient wake structures driven by dominant instability mechanisms present in the wake. It has been found that manipulation of these largescale flow structures is an effective approach to reduce form drag [16-23]. Therefore, understanding of the instability mechanisms prevailing in the wake is key to devise optimal wake-flow control strategies. Bluff-body wakes exhibit strong shear flows that are prone to instabilities. Global dynamics of these flows strongly depend on the characteristics of local instabilities which can be either convective or absolute [24]. A parallel shear flow is convectively unstable if the growing wavepacket originating from an impulsive source localized in time and space is swept away by advection. The shear flow is said to be absolutely unstable if the growing wavepacket displays nontrivial dynamics without any external impulse and grows in situ to contaminate the entire flow medium [25-27].

Studies investigating instabilities and resulting flow structures in the wake of bluff bodies have tended to involve geometries that are either axisymmetric (circular-diskshaped and bullet-shaped bodies) or planar symmetric in the plane perpendicular to the undisturbed flow direction [28-30]. Although wakes behind axisymmetric bluff bodies appear to have attracted somewhat less interest than their planar-symmetric counterparts, the wake of spheres is an exception in this regard. Early flow visualizations performed by Taneda [31] showed that flow separation occurring at a Reynolds number of about 24 based on the sphere diameter gives rise to a stationary vortex ring that remains attached to the sphere. The steady axisymmetric wake

of the sphere remains stable up to a Reynolds number of 210, at which point the 
wake experiences a regular symmetry-breaking bifurcation resulting in a steady nonaxisymmetric wake [32]. This steady non-axisymmetric wake preserves a symmetry plane and displays two counter-rotating streamwise vortices emanating from the end of the recirculation zone in the near-wake of the sphere [33,34]. At a Reynolds number of 270 , the wake becomes unsteady and exhibits time-periodic vortex shedding through a supercritical Hopf bifurcation described by the Stuart-Landau equation [35-37]. In this regime, the basic planar-symmetric wake configuration is made of a succession of interconnected vortex loops shown schematically in Figure 3.1. In

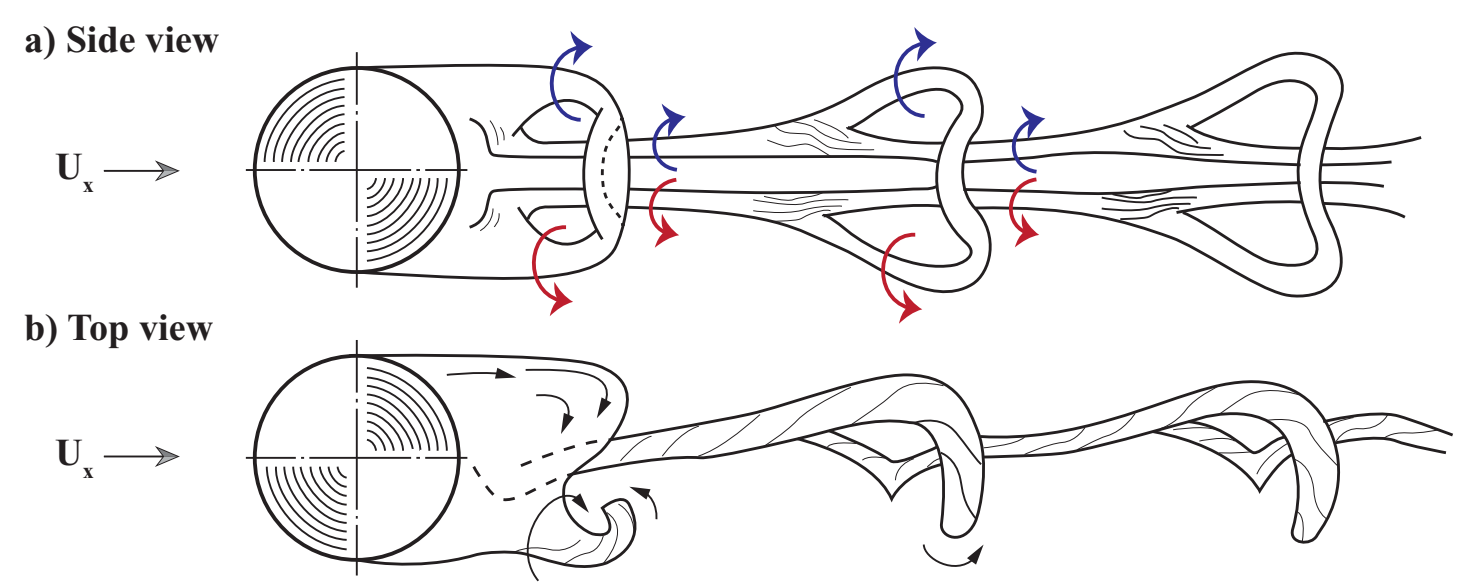

Figure 3.1: Dominant flow structures in the wake of sphere at $\operatorname{Re}_{\mathrm{D}}=\mathbf{1 0 0 0}$ (adapted from Achenbach [38])

this instability mode, generation of the interconnected vortex loops is attributed to the self-sustained oscillations of the vortex ring attached to the sphere [39]. Kim \& Durbin [40] showed that the formation frequency of the vortex loops corresponds to a Strouhal number of 0.2 , and is almost independent of Reynolds number. As the Reynolds number is increased and the wake flow transitions into a more chaotic state, the details of the wake dynamics and the cause-and-effect relationships become less clear. Above a Reynolds number of about 400, the symmetry plane of the wake of the sphere is observed to start to rotate somewhat randomly with an approximate rotational speed corresponding to $1.4 \mathrm{U}_{\infty} / \mathrm{D}$ [41]. Flow visualizations by Sakamoto \& Haniu [42] suggest the formation of a streamwise train of vortex tubes or rings 
in the immediate vicinity of the sphere via the Kelvin-Helmholtz (K-H) instability mechanism starting at a Reynolds number of about 800. The formation frequency of these vortical structures corresponds to a Strouhal number in the range of 2 to $8[40,42]$. Coexistence of the shedding activities at the lower and higher frequencies was confirmed in the experiments of Kim \& Durbin [40] and Berger et al. [43]. Unlike the lower-frequency mode, the Strouhal number of the higher-frequency shedding increases proportional to $R e_{D}{ }^{0.75}$ as the shear layer progressively becomes unstable to smaller wavelengths $[40,44]$. As the Reynolds number is increased beyond 1000, the wake develops a helical vortex structure that is associated with a local absolute instability in the near wake region $[31,43,45]$. The helix structure originates from the location of the pressure fluctuation maxima which occurs just upstream of the rear stagnation point of the recirculation zone. Recent studies by Pier [46] and Skarysz et al. [47] indicate that this flow regime exhibits both low- and high-frequency helical states with the wake transitioning back and forth from one state to the other. At a Reynolds number of 370,000, the wake of the sphere undergoes another transition resulting in a shrunk recirculation zone, leading to a sharp reduction in drag $[31,38]$. This new wake structure displays two counter-rotating streamwise vortices extending downstream with a planar symmetry. The underlying physical mechanisms of this transition remain unclear.

The wake development for circular disks and other axisymmetric bluff-body shapes appear to follow a pattern remarkably similar to that of a sphere. In the case of a circular disk, the direct numerical simulation (DNS) studies of Fabre et al. [48] and Meliga et al. [49] show that three successive bifurcations occur in the low-Reynolds range: a regular bifurcation at a Reynolds number of 117 yielding a steady three-dimensional wake with planar symmetry; a Hopf bifurcation at a Reynolds number of 123 transitioning the wake to a state of periodic vortex shedding without planar symmetry; and, a third bifurcation at a Reynolds number of 140 producing an unsteady planar symmetric wake. Flow visualizations conducted by Berger et al. [43] and Marshall \& Stanton [50] confirm the close similarity of the wake configurations and transition mechanisms of spheres and circular disks. Sevilla \& Martinez-Bazan [45], Bury \& 
Jardin [51] and Rigas et al. [52,53] describe the wake physics for bullet-shaped axisymmetric bluff bodies through similar wake structures and transition mechanisms.

Amongst planar-symmetric bluff bodies, the 2D (infinitely long) circular cylinder of uniform diameter has been studied most extensively due to its frequent use in engineering applications, relative geometric simplicity, and particularly interesting wake dynamics due to the absence of fixed flow separation points. The experiments of Roshko [54] and Taneda [7] showed that, up to a Reynolds number of 49 , the cylinder wake consists of a steady recirculation region driven by two vortices maintaining a planar-symmetric wake pattern. Above this Reynolds number value, the wake becomes unstable, which first manifests itself at the downstream end of the recirculation zone via a supercritical Hopf bifurcation [55]. The resultant primary Karman vortex shedding is driven by periodic, self-excited limit-cycle oscillations of the near wake [56]. These self-excited global oscillations of the entire near wake have been closely associated with a local absolute instability in several studies [25, 57-61]. Flow visualizations of Williamson [62], Konig et al. [63] and Hammache \& Gharib [64] indicate that the end conditions of the cylinder dictate whether the vortices are shed in parallel mode or in oblique mode involving a chevron-patterned shedding along the span of the cylinder with typical oblique angles of $15-20^{\circ}$. At a Reynolds number of 190, the two-dimensional wake undergoes a hysteretic transformation to three-dimensional flow, giving rise to the first of a sequence of two vortex shedding modes, commonly referred to as modes $\mathrm{A}$ and B $[65,66]$. These modes are illustrated schematically in Figure 3.2. The mode-A instability deforms the primary Karman vortices into streamwise vortex pairs with a spanwise wavelength of 3 to 4 cylinder diameters [67]. This development has been suggested as the underlying cause of the spanwise dislocations of the Karman vortices [68]. The same instability mode is indicated as the initial transition mechanism for a range of other planar symmetric bluff bodies, ranging from 2D cylinders of square cross section to plates of large span and streamlined noses [69,70]. Over a Reynolds number range of 230 to 250, a gradual energy transfer from the streamwise vortices of mode A shedding to the mode $\mathrm{B}$ shedding gives rise to generation of finer-scale streamwise vortices. At the end of this Reynolds number range, the wake becomes 

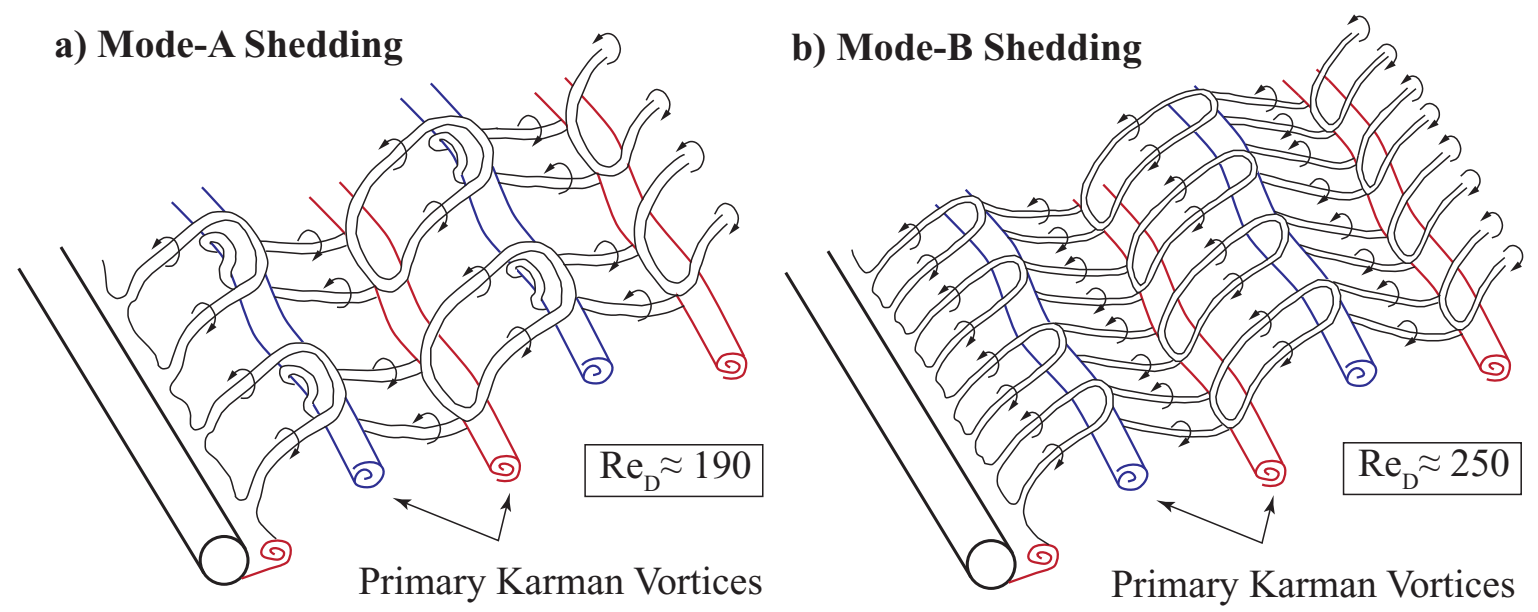

Figure 3.2: Instability modes of $\mathrm{A}$ and $\mathrm{B}$ in the wake of a $2 \mathrm{D}$ cylinder

completely unstable to mode B shedding, with the wake dominated by streamwise vortices of about one-diameter spanwise spacing [88]. Williamson [72] showed that mode A involves streamwise vortices with one sense of rotation located in a staggered arrangement from one braid region to next, whereas mode B has an in-line arrangement of streamwise vortices of the same sense of rotation, as illustrated in Figure 3.2. There is evidence that mode A may be driven by an elliptical instability of the primary vortex core during the formation of the vortex, while mode B may be associated with a hyperbolic instability of the braid region prevailing between streamwise-adjacent primary Karman vortices [73].

The flow visualizations of Gerrard [56] show that relative positioning of the primary Karman vortices between the port and starboard sides of the cylinder varies between 0.2 to 1 cylinder diameter in the near wake. As the Reynolds number is increased from 1000 to 120,000, upon separation from the cylinder surface, the shear layer deforms via the Kelvin-Helmholtz mechanism to form a streamwise train of vortices with a streamwise-wavelength Strouhal number that is proportional to $R e_{D}^{0.5}[74]$. Within this regime, the time-mean formation length of the recirculation region has been observed to decrease while the drag is increased [75]. The drag force on the cylinder is observed to decrease with Reynolds number, starting at a Reynolds number value of about 120,000 [75]. Furthermore, in the Reynolds number range of about 330,000 to 423,000 , the flow separation on the starboard 
and port sides of the cylinder develop an alternating pattern $[76,77]$. Unlike the $1000-120,000$ Reynolds number range, rather large transient lateral forces $\left(\mathrm{C}_{\mathrm{L}} \approx 1\right)$ occur, while the formation length of the mean recirculation region decreases through partial reattachment of separating shear layers, which provides a plausible explanation for the observed drag reduction in this Reynolds number range $[78,79]$.

Other types of planar symmetric bodies include rectilinear bodies, i.e. bodies with a rectangular base, which display somewhat different wake physics than those of 2D planar symmetric bodies due to their finite span. The most well-known of such geometries is the Ahmed body which was originally studied as a simplified roadvehicle model to investigate vehicle aerodynamics [80]. This body has mostly been studied in ground proximity. Figure 3.3 illustrates the wake development of a squareback Ahmed body. For Reynolds numbers of order of 100 based on the model height, the wake of this body has been observed to be steady and symmetric with respect to

a) $\operatorname{Re}_{\mathrm{H}} \approx 100$

Top view

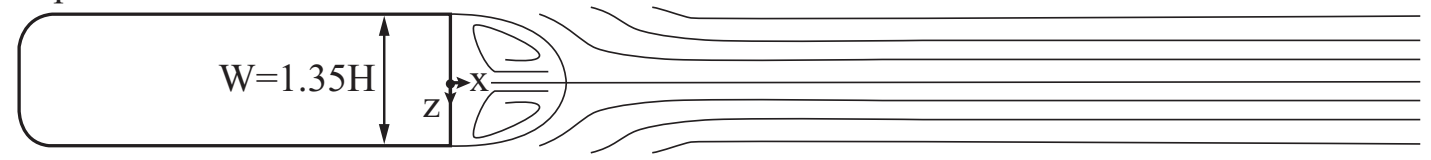

Side view

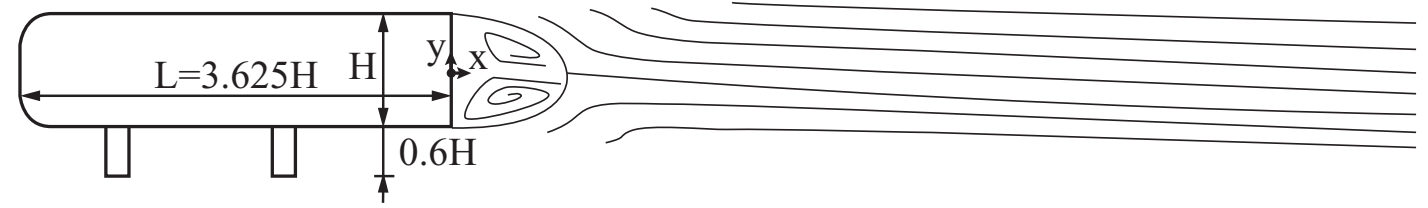

b) $\operatorname{Re}_{\mathrm{H}} \approx 365$

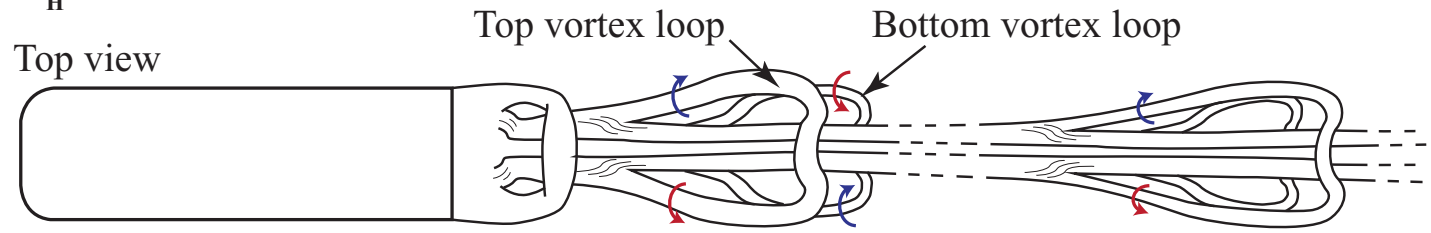

Side view

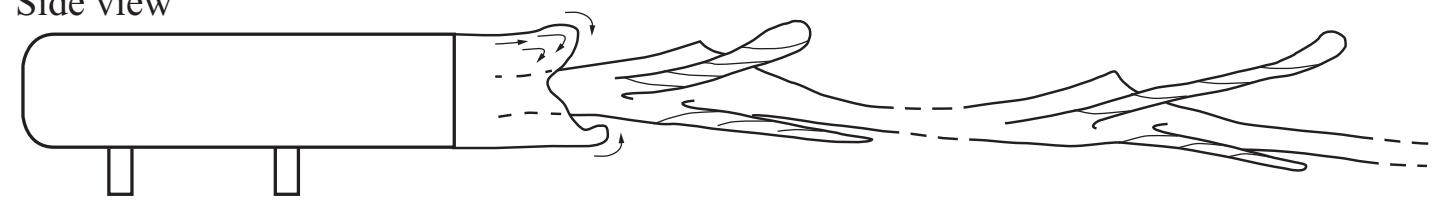

Figure 3.3: Wake development behind the Ahmed body in ground proximity 
the $\mathrm{x}-\mathrm{y}$ plane, while there exists a slight top-bottom asymmetry due to the presence of the ground [81]. At about a Reynolds number of 340, the wake undergoes a pitchfork bifurcation leading to periodic oscillations in the plane of symmetry, while the symmetry is still preserved [82]. With further increase of the Reynolds number, the flow becomes unsteady and the wake displays periodic vortex shedding associated with alternate vortex loops that are shed from the top and bottom shear layers [81].

A recent subject of particular interest regarding three-dimensional bluff-body wakes is flow bistability that is illustrated as an example in the wake of a squareback Ahmed body in Figure 3.4. It refers to random flipping of the wake between two asymmetric states via a reflectional-symmetry-breaking (RSB) instability mode, even under nominally axisymmetric or planar symmetric flow conditions [83,84]. Recent studies investigated this phenomenon for bodies such as the sphere [41,85-87], the
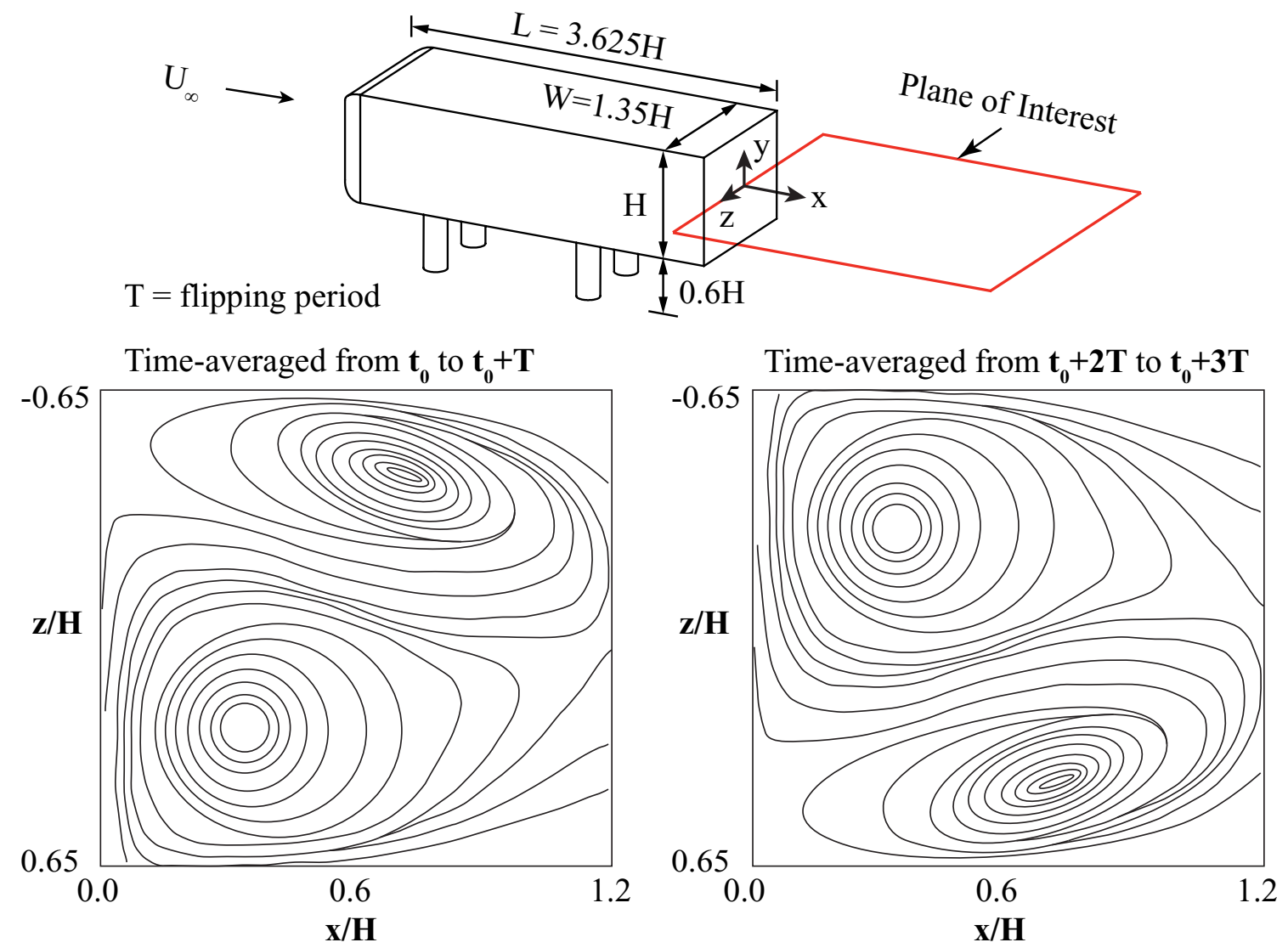

Figure 3.4: Streamline patterns illustrating the wake bistability of a square-back Ahmed body in ground proximity at $\operatorname{Re}_{\mathbf{H}} \approx 10^{5}[89]$ 
circular disk $[49,86]$, bullet-shaped bodies $[45,51,53]$, the 2D circular cylinder $[75,88]$, and the Ahmed body [81,90-93]. Common to these studies is that the wake is instantaneously asymmetric at one of the two mirrored bifurcated states while the flipping between these two states leads to a symmetric wake when averaged over a sufficiently long time period [89]. The period of flipping is found to be several orders of magnitude larger than the typical period of time-periodic dynamics such as vortex shedding that prevail in the near wake [89]. For axisymmetric bodies, the long-time azimuthal position of the plane in which the RSB mode develops has been observed to be random [87]. In contrast, for bodies with a rectangular base, the RSB mode is noted to develop for a finite range of base aspect ratios (e.g. 1.1 to 1.65 for $R e_{H}=45,000$ [82]), and is observed to develop within the geometric symmetry plane that is parallel to the longer dimension of the base as illustrated in Figure 3.4 [94]. For ground clearance values less than $0.135 \mathrm{H}$, the RSB instability mode driving the wake bistability of the Ahmed body (Figure 3.4) is suppressed due to the effective aspect ratio of the model being altered by the presence of the ground. The presence of RSB modes in the wake has been suggested to promote higher form drag on bluff bodies [90].

In the 1970s, significant drag reduction was realized through rounding the corners of box-shaped ground vehicles such as delivery vans and transport trucks, yielding more streamlined body shapes [95]. Additional geometric modifications to affect form drag included add-on devices such as cab top/side extensions, trailer side-skirts, boattails and trailer vortex generators [96-102]. In certain combinations, up to 6 percent drag reduction has been achieved with these devices [103]. In the boat-tailing concept, a tapered fairing mounted to the rear of the vehicle guides the wake flow inwards, increasing the average pressure acting on the backface of the trailer. Recent studies focusing on the wake instabilities of bluff bodies have suggested introduction of a base cavity to a square-back Ahmed body to attenuate the wake bistability, and thereby promoting a significant reduction in form drag $[90,104,105]$. The proposed utilization of a base cavity on an Ahmed body resembles the configuration involving boat-tails.

A trailing-edge geometry that has been used with great success for mixing coflowing streams is the lobed mixer. Considering the wake region next to the base of a bluff body as a "slow-moving stream", in principle the lobed mixer may be effective 
in promoting mixing between the wake and its surroundings. A typical lobed trailing edge with geometric parameters that are pertinent to its mixing effectiveness is illustrated in Figure 3.5.
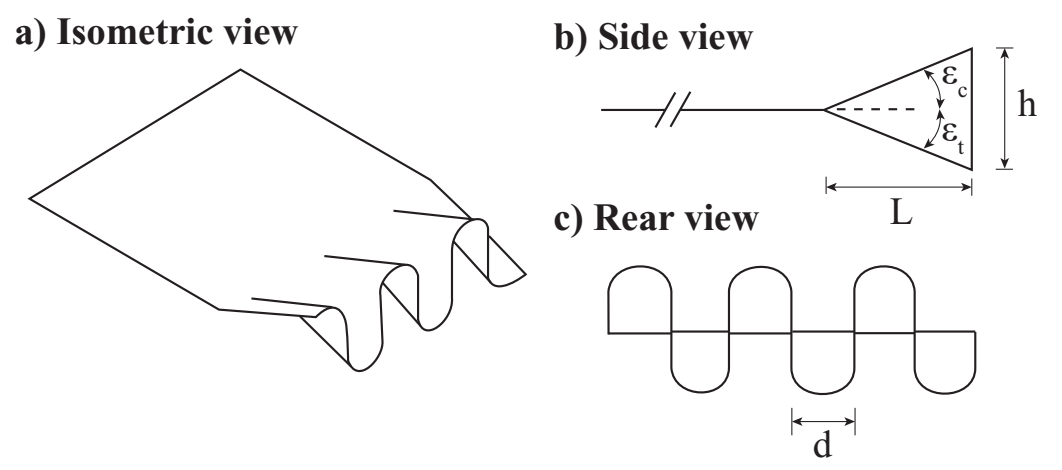

$$
\begin{aligned}
& \mathrm{L}=\text { Length } \\
& \mathrm{h}=\text { Height } \\
& \mathrm{d}=\text { Width } \\
& \varepsilon_{\mathrm{c}}=\text { Outer penetration angle } \\
& \varepsilon_{\mathrm{t}}=\text { Inner penetration angle }
\end{aligned}
$$

\section{Figure 3.5: Schematic of a generic lobed mixer}

The enhancement in mixing in the presence of lobes is primarily attributed to the increase in interfacial area and the formation of coherent streamwise vortices [106,107]. Paterson [108] performed the first detailed study of the lobed-mixer flow field and revealed the existence of the streamwise vortices that develop from radially oriented secondary flows prevailing along the sidewalls of the lobes. Werle et al. [109] and Eckerle et al. [110] observed the streamwise vortices to have a diameter on the order of the lobe width upon formation. The strength of the streamwise vortices has been found to be dependent on the inner and outer penetration angles of the lobes [111], the velocity ratio of the coflowing fluids $[112,113]$ and the thickness of the boundary layer formed in the lobe troughs [113]. The boundary layer measurements of Skebe et al. [114] suggest that relatively thinner boundary layers prevail along the lobe crest while the opposite is true for the boundary layer at the trough. This trend is the result of streamwise acceleration of the boundary layer along the lobe crest and streamwise deceleration along the trough, supplemented by redistribution of the boundary layer fluid between the crest and trough regions prompted by the local cross-stream pressure gradients. Skebe et al. [114] and Barber et al. [113] observe that having the sidewalls of the lobes parallel to each other rather than a lobe geometry that resembles a sine wave reduces the extent of migration of boundary-layer 
fluid towards the trough. This, in turn, is observed to yield improved mixing performance downstream of the trailing edge plane. O'Sullivan et al. [115] explain this trend in the mixing performance based on a reduction in the effective penetration angle of the lobes that would result from a thicker boundary layer at the trough, thereby yielding weaker streamwise vortices. The boundary layers developing along the walls of the lobed mixer may be laminar or turbulent depending on the extent of disturbances in the flow environment and the flow Reynolds number. Studies of Brinkerhoff et al. [116] and Ooba et al. [117] have shown that small-scale turbulence present in the freestream flow or developed in shear layers along the lobed-mixer walls influences the boundary layer development along the walls as it appears to energize the separated flow, allowing it to reattach shortly after separation, whereas reattachment does not occur in the laminar scenario. In the absence of boundary layer separation, the pressure losses associated with lobed mixers are due to skin friction and the mixing out of velocity non-uniformities downstream of the trailing edge [118]. Measurements of Underwood and Waitz [119] indicate that mixing losses are the dominant contributor to total-pressure loss.

The primary mechanism driving mass and momentum exchange between coflowing streams of fluid is the Kelvin-Helmholtz instability that is promoted inviscidly by the inflectional axial velocity profile of the shear layer immediately downstream of the lobes. Spanwise-oriented vortices, also known as normal vortices owing to their orientation relative to the primary flow direction, are generated as a result of the roll-up of the free shear layer at the interface of the two streams through this instability mechanism. The streamwise spacing of these normal vortices tends to double with sufficient downstream distance because of a secondary inviscid instability mode that results in vortex pairing, similar to what has been observed in planar free-shear layers [120,121]. The observations of McCormick and Bennett [122] indicate that the normal vortices are continuously connected along the span of the convoluted freeshear layer immediately downstream of the lobed-mixer trailing edge. As the normal vortices convect downstream, the relatively larger amount of lower-momentum fluid prevailing in the trough region causes the trough segments of the normal vortices to be progressively stretched in the upstream direction [116]. The free-shear layer shedding 
off the trailing edge of the sidewall of the lobe contains vorticity with a streamwise component, and rolls into a streamwise vortex under its self-induced velocity field. The mutual interaction of the streamwise and normal vortices results in their deformation as shown schematically in Figure 3.6, with the normal vortices bulging in the trough and crest regions.

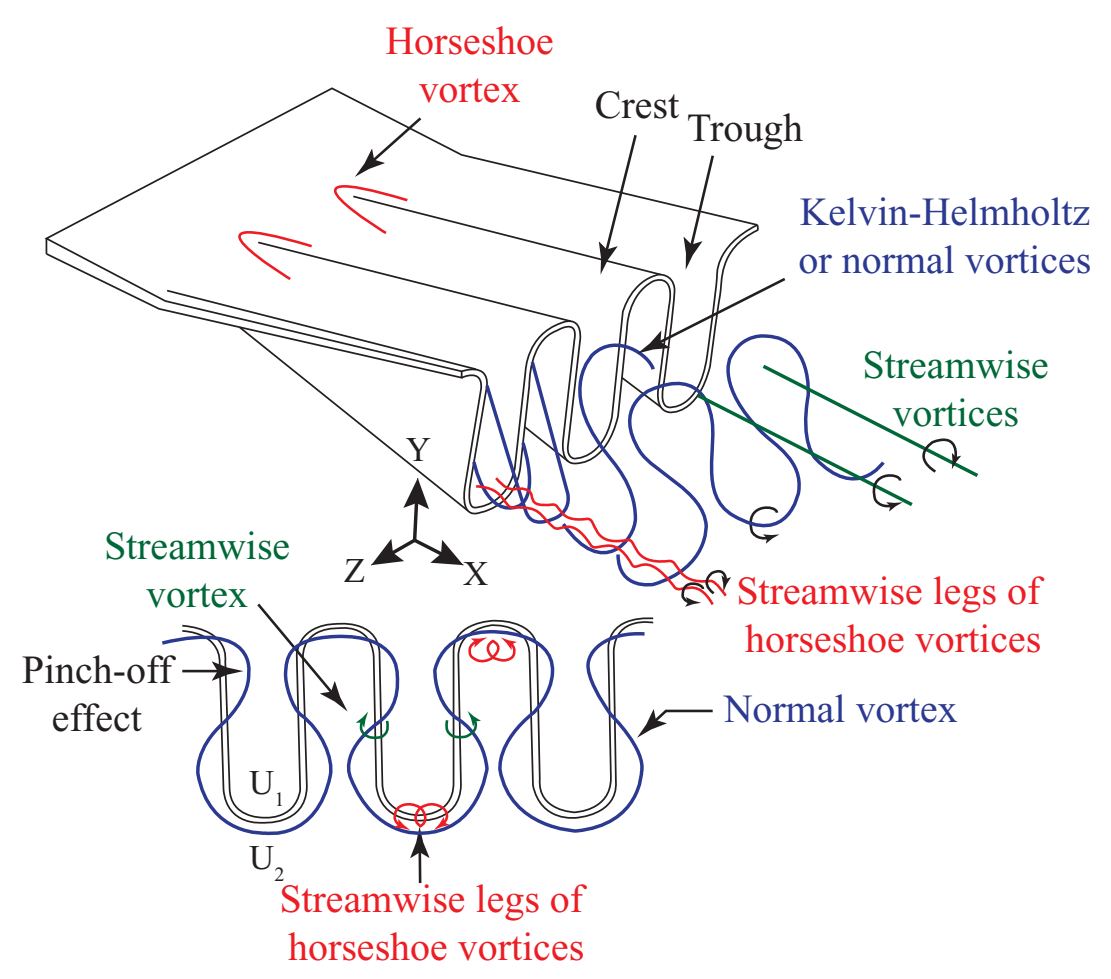

Figure 3.6: Schematic of the vortical structures downstream of the lobedmixer (adapted from McCormick and Bennett [122])

With streamwise distance, these bulges grow until the normal vortices are broken apart in the regions of pinch-off, triggering the breakdown of the coherent vortical structures into smaller scale turbulence. Measurements of Glasuer et al. [123], Ukeiley et al. [124] and $\mathrm{Hu}$ et al. [125] suggest that the collapse of the pinched-off normal vortices onto themselves, which occurs at multiple lobe heights downstream of the trailing edge, is the main reason for enhanced turbulence produced by the lobed trailing edge. Moreover, the existence of a counter-rotating streamwise vortex pair in the troughs of the lobes is commonly observed downstream of the discharge-plane [122], which are the legs of horseshoe vortices that develop as the upstream wall boundary 
layer is wrapped around the lobe peaks. These vortices seem to be relatively inconsequential to the mixing process because the close proximity of the streamwise legs of the horseshoe vortices from neighboring lobes results in their relatively rapid mutual destruction owing to their opposite senses of rotation. These flow physics and mixing performance of the lobed mixer are known to be influenced by compressibility. This subject is not reviewed here given the focus of the present study on applications involving incompressible flow speeds. For completeness of the review of this subject, the effects of compressibility on lobed mixer performance are summarized in Appendix D.

Combining the lobed-mixer concept with the boat-tail concept may yield a tail geometry with improved performance than the configurations where these devices are utilized separately. Investigating the effectiveness of this lobed-tail configuration in comparison to the plain boat-tail configuration constitutes the primary objective of the present study. 


\section{Chapter 4}

\section{Experimental Setup}

\subsection{Geometry of Bluff-Body Model and Tails}

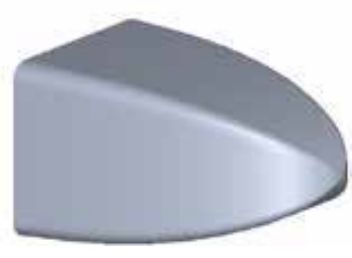

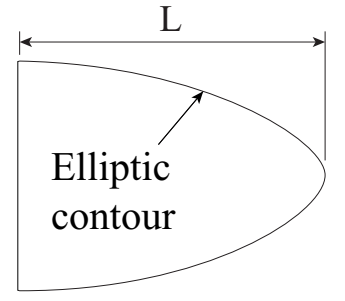

a) Side view

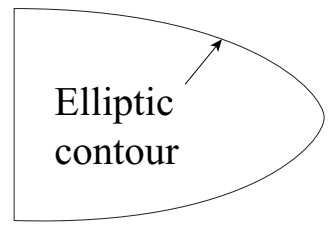

c) Bottom view

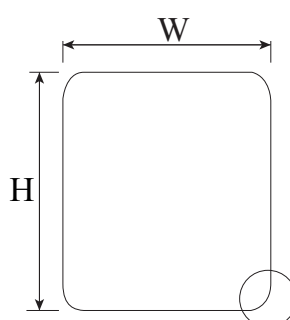

b) Front View

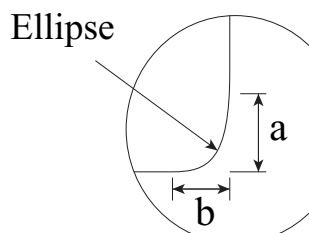

d) Detail view

Figure 4.1: Schematic of the bluff-body model

Table 4.1: Geometric parameters of the bluff-body model

\begin{tabular}{lc} 
Model length, $L$ & $303.3 \mathrm{~mm}$ \\
Model height, $H$ & $210.0 \mathrm{~mm}$ \\
Model width, $W$ & $182.0 \mathrm{~mm}$ \\
Hydraulic diameter, $D_{h}=2 H W /(H+W)$ & $195.0 \mathrm{~mm}$ \\
Projected frontal area, $A$ & $37892 \mathrm{~mm}^{2}$ \\
Model aspect ratio, $A R=H / W$ & 1.15 \\
Fillet major-axis length, $a$ & $21.0 \mathrm{~mm}$ \\
Fillet minor-axis length, $b$ & $18.2 \mathrm{~mm}$ \\
\hline
\end{tabular}

The geometry of the idealized bluff-body model considered in the present study is shown in Figure 4.1, with the noted parameters quantified in Table 4.1. The aspect ratio of the trailing-edge cross section is set to that of the standard truck trailers in 
North America [103]. The size of the model was dictated by the size of the windtunnel test section, and the sensitivity and accuracy of the load balance of the wind tunnel that is used to quantify the relatively small changes in the drag force of the bluff-body model. A notably more streamlined upstream portion was chosen for the

a)
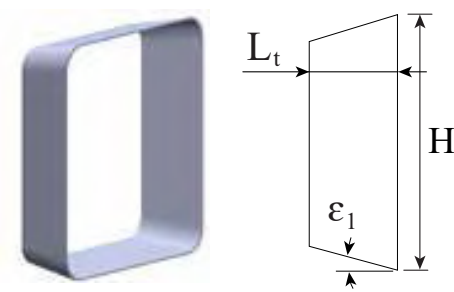

Side view

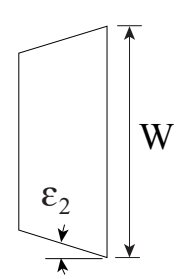

Top view b)

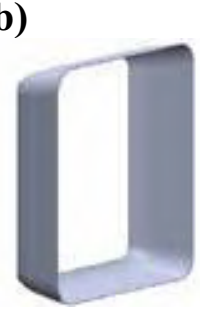

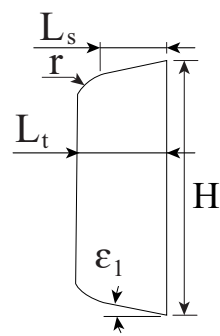

Side view

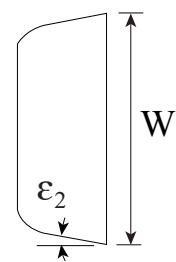

Top view

c)
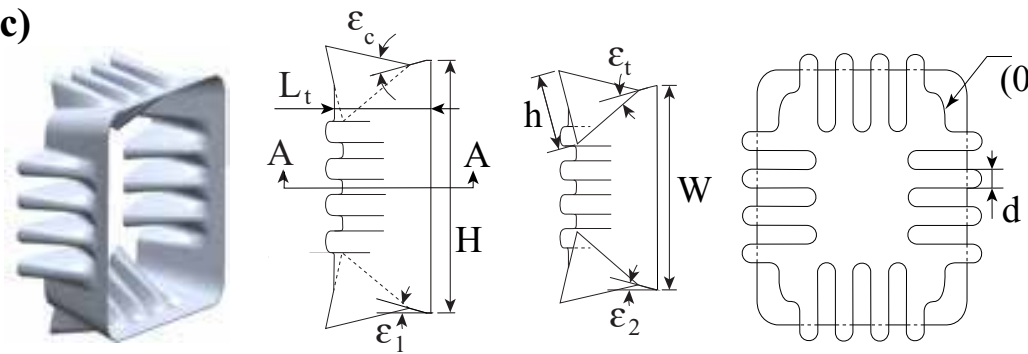

Ellipse $(0.05 \mathrm{~W}, 0.07 \mathrm{H})$

Side view

Section A-A

Rear view

Figure 4.2: Schematics of a) straight tail b) curved tail and c) lobed tail

Table 4.2: Geometric parameters of the tails

\begin{tabular}{lccccc}
\hline Tail Type & $\begin{array}{c}\text { Short } \\
\text { Straight }\end{array}$ & $\begin{array}{c}\text { Long } \\
\text { Straight }\end{array}$ & $\begin{array}{c}\text { Long } \\
\text { Curved }\end{array}$ & $\begin{array}{c}\text { Long } \\
\text { Large-Lobed }\end{array}$ & $\begin{array}{c}\text { Long } \\
\text { Small-Lobed }\end{array}$ \\
\hline Tail length, $L_{t}$ & $42.0 \mathrm{~mm}$ & $84.0 \mathrm{~mm}$ & $84.0 \mathrm{~mm}$ & $84.0 \mathrm{~mm}$ & $84.0 \mathrm{~mm}$ \\
Tail height, $H$ & $210.0 \mathrm{~mm}$ & $210.0 \mathrm{~mm}$ & $210.0 \mathrm{~mm}$ & $210.0 \mathrm{~mm}$ & $210.0 \mathrm{~mm}$ \\
Tail width, $W$ & $182.0 \mathrm{~mm}$ & $182.0 \mathrm{~mm}$ & $182.0 \mathrm{~mm}$ & $182.0 \mathrm{~mm}$ & $182.0 \mathrm{~mm}$ \\
Straight segment length, $L_{s}$ & $42.0 \mathrm{~mm}$ & $84.0 \mathrm{~mm}$ & $66.8 \mathrm{~mm}$ & $13.8 \mathrm{~mm}$ & $62.0 \mathrm{~mm}$ \\
Radius of curvature, $r$ & - & - & $30.0 \mathrm{~mm}$ & - & - \\
Lobe height, $h$ & - & - & - & $66.0 \mathrm{~mm}$ & $18.0 \mathrm{~mm}$ \\
Lobe width, $d$ & - & - & - & $16.5 \mathrm{~mm}$ & $5.0 \mathrm{~mm}$ \\
Outer penetration angle, $\epsilon_{c}$ & - & - & - & $25.0 \mathrm{deg}$ & $25.0 \mathrm{deg}$ \\
Inner penetration angle, $\epsilon_{t}$ & - & - & - & $25.0 \mathrm{deg}$ & $25.0 \mathrm{deg}$ \\
Top/bottom-tail angle, $\epsilon_{1}$ & $11.0 \mathrm{deg}$ & $11.0 \mathrm{deg}$ & $11.0 \mathrm{deg}$ & $13.0 \mathrm{deg}$ & $11.0 \mathrm{deg}$ \\
Side-tail angle, $\epsilon_{2}$ & $13.0 \mathrm{deg}$ & $13.0 \mathrm{deg}$ & $13.0 \mathrm{deg}$ & $13.0 \mathrm{deg}$ & $13.0 \mathrm{deg}$ \\
\hline
\end{tabular}


bluff-body model compared to the shape of a tractor trailer to ensure dominance of the wake dynamics in the overall aerodynamic drag.

The tail geometries that were investigated are presented in Figure 4.2, with the primary geometric parameters listed in Table 4.2. When the tails are scaled up to the dimensions of truck trailers used in North America, they remain within the permissible space envelope stipulated by the Canada Motor Vehicle Safety Standard $223[5]$.

The bluff-body model and its tails have 1.5-mm-thick walls and were constructed out of acrylonitrile butadiene styrene (ABS) plastic using a Dimension BST400 rapid prototyping machine. Dimensional tolerances of the model and its tails were within $\pm 0.1 \mathrm{~mm}$ with a surface roughness of about $20 \mu \mathrm{m}$ dictated by the resolution of the rapid prototyping machine.

\subsection{Wind Tunnel Setup}

The experiments were performed in a closed-circuit wind tunnel that is shown in Figure 4.3. The tunnel makes use of a contraction ratio of 9:1 and flow-conditioning

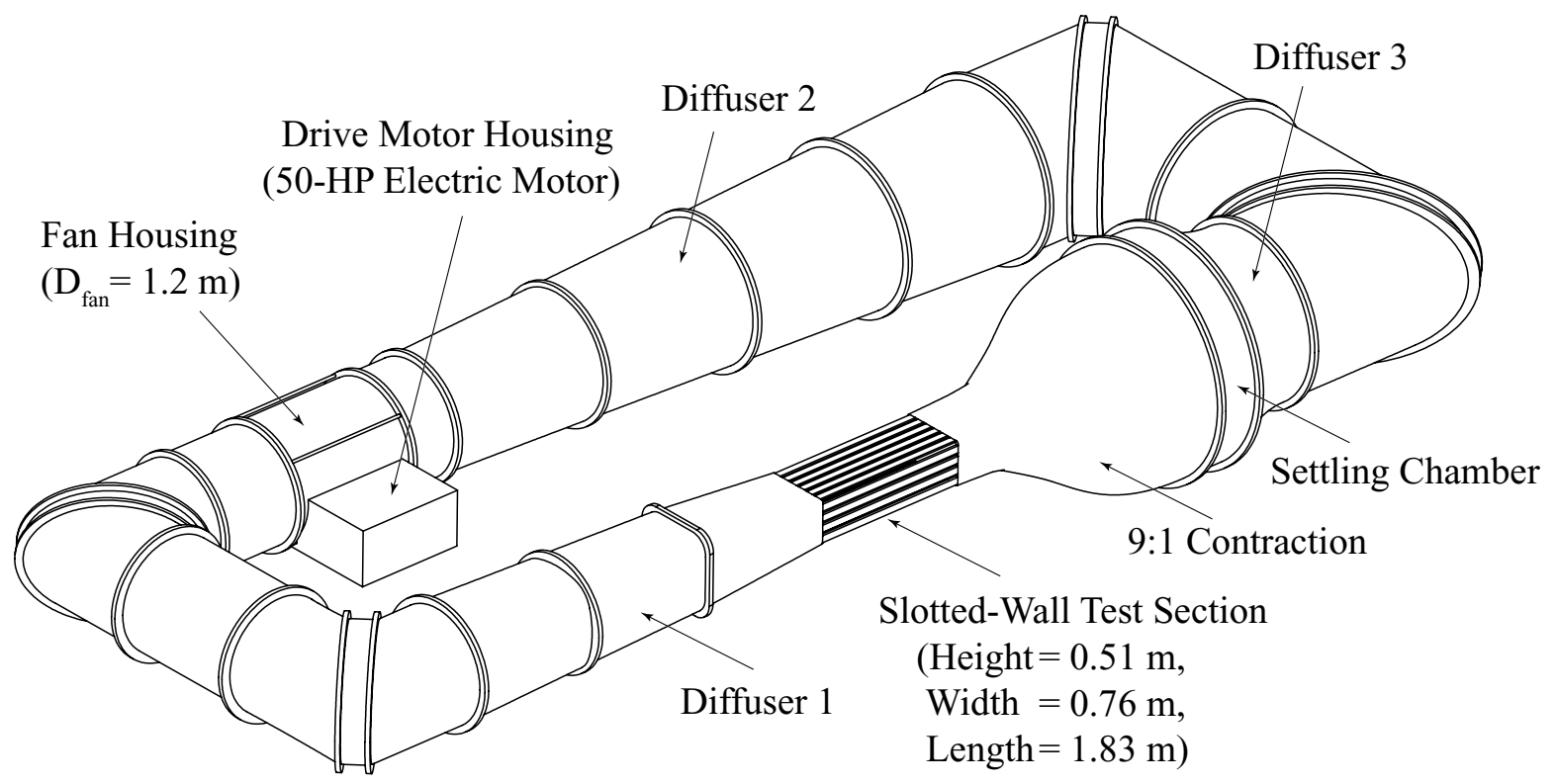

Figure 4.3: Schematic of wind-tunnel facility 
screens located inside settling chamber to yield a test section inflow with a velocity uniformity that is within $\pm 2 \%$ of the spatially-averaged value, and a turbulence intensity of less than $0.5 \%$.

The test model was installed in a test section with streamwise-slotted walls of $35 \%$ open area as illustrated in Figure 4.4. The use of slotted walls on wind-tunnel test sections is a well-established practise to reduce aerodynamic wall interference while allowing the use of test models with relatively larger percentage blockage of the flow area [126-129]. The present slot configuration was based on well-established design guidelines [130-140]. The model size as presented in Figure 4.1 and Table 4.1 yields a $10 \%$ aerodynamic blockage in this test section.

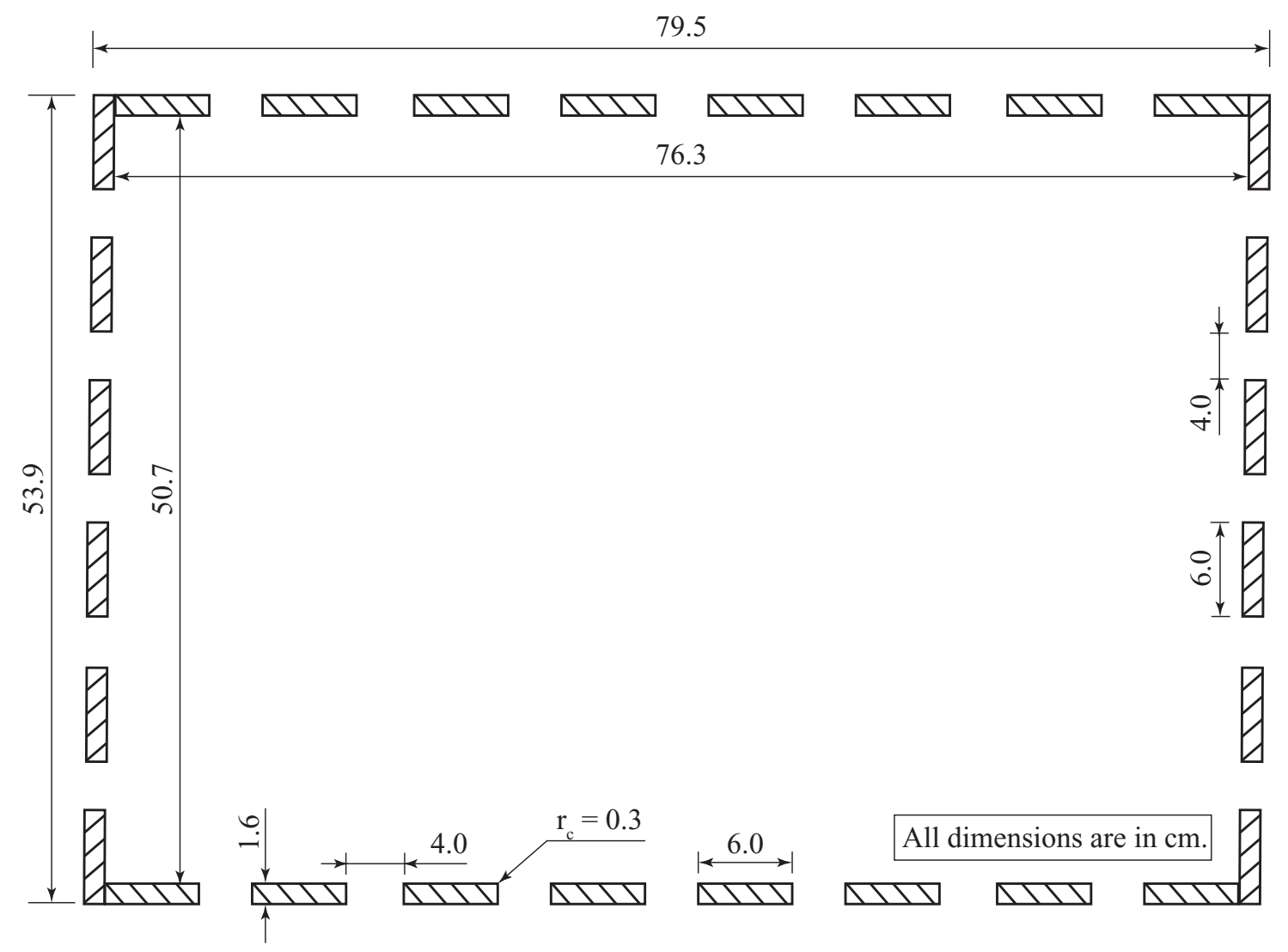

Figure 4.4: Cross-sectional view of the slotted-wall test section

The model was mounted with its centerline aligned with the longitudinal axis of the test section, with the model centerline located $25.6 \mathrm{~cm}$ from the ceiling and centered between the two side walls. Longitudinally, the model was placed such that, 
without the tails, its trailing edge was at a distance of $4 \mathrm{D}_{\mathrm{h}}$ from the test-section inlet. For all of the tail configurations considered, the trailing edge of the model remained farther than $5 \mathrm{D}_{\mathrm{h}}$ from the downstream end of the test section. This allowed the near-wake of the test model to develop unaffected by the pressure recovery in the diffuser section downstream of the test section. The model was held in place within the test section with the aid of three streamlined struts that are connected to a force balance installed above the test section. The support system was developed by Mr. C. Pretorius, a MASc student in the author's research group. The installation is shown in Figure 4.5. The streamlined struts were determined to produce a parasitic drag that is less than $5 \%$ of the drag force on the model, and this was accounted for in the processing of the measurement results.

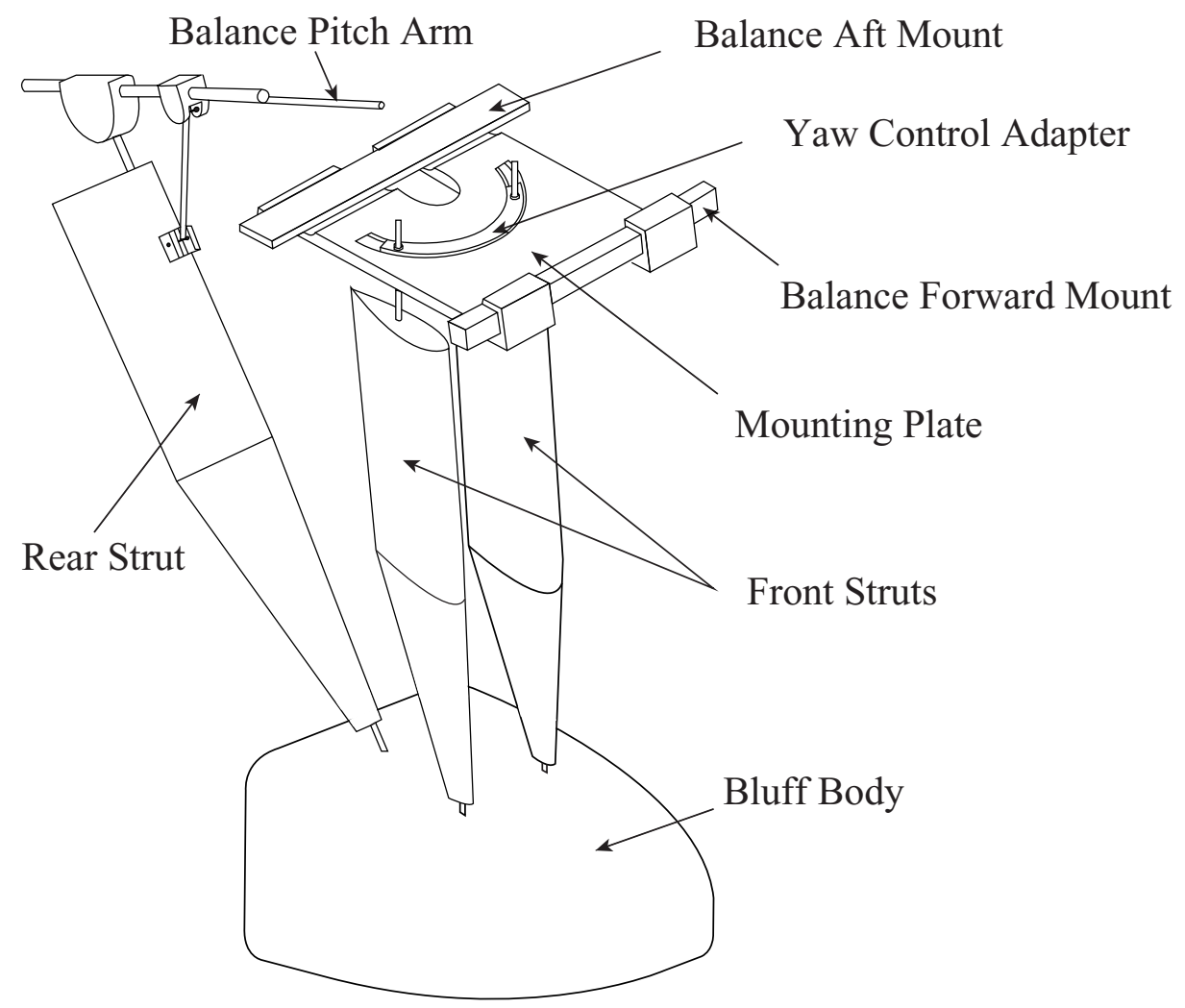

Figure 4.5: Schematic of model installation in the test section 


\subsection{Operating Conditions}

The experiments were performed at a Reynolds number of $128,000 \pm 700$ based on the undisturbed freestream velocity and hydraulic diameter of the model (Table 4.1). This corresponding Reynolds number value is an order of magnitude smaller than those that correspond to the operating range of full-scale trucks. Nonetheless, for this range of Reynolds numbers, the boundary layers and wake of the present bluff body are expected to be relatively insensitive to Reynolds number, hence the measured results remain relevant to the aerodynamics of full-sized tractor trailers.

\subsection{Instrumentation and Data Acquisition}

The experimental aerodynamic drag coefficient, which is the parameter of interest for the current investigation, is defined as:

$$
C_{D}=\frac{D}{\frac{1}{2} \rho U_{\infty}^{2} A}
$$

where $D$ is aerodynamic drag force, $\rho$ is air density, $U_{\infty}$ is test-section undisturbed centerline velocity and $A$ is the projected frontal area of the test model. The freestream velocity at the inlet centerline of the test section was correlated to the contraction pressure difference of the wind tunnel. The contraction pressure difference was measured with an inclined manometer. The resultant velocity is deemed to be accurate to within $\pm 3 \%$. The air density was quantified using the test section undisturbed static pressure that was equal to ambient pressure measured with a barometer, and the air temperature in the test section measured with a thermocouple. During the experiments, the resultant density is deemed to be accurate to within $\pm 2 \%$. The drag-force load cell on the force balance mounted above the test section was calibrated with dead weights prior to the experiments. The measured drag force of the model, after it was corrected for the parasitic drag of the struts, is deemed to be accurate to within $\pm 4 \%$. Propagating the stated uncertainties for the drag force, density and reference velocity, through Equation 4.1 yields an uncertainty of $\pm 6 \%$ for the drag coefficient. 


\section{Chapter 5}

\section{Computational Models and Computational Method}

\subsection{Computational Models}

\subsubsection{Computational Domain and Boundary Conditions}

The computational domain and its boundary types for the present flow simulations are shown in Figure 5.1. The domain is sized notably larger than the region of interest to allow for straight forward implementation of boundary conditions on the boundaries identified in the figure while avoiding any non-physical influence of these boundary conditions on the flow in the vicinity of the model and its wake.

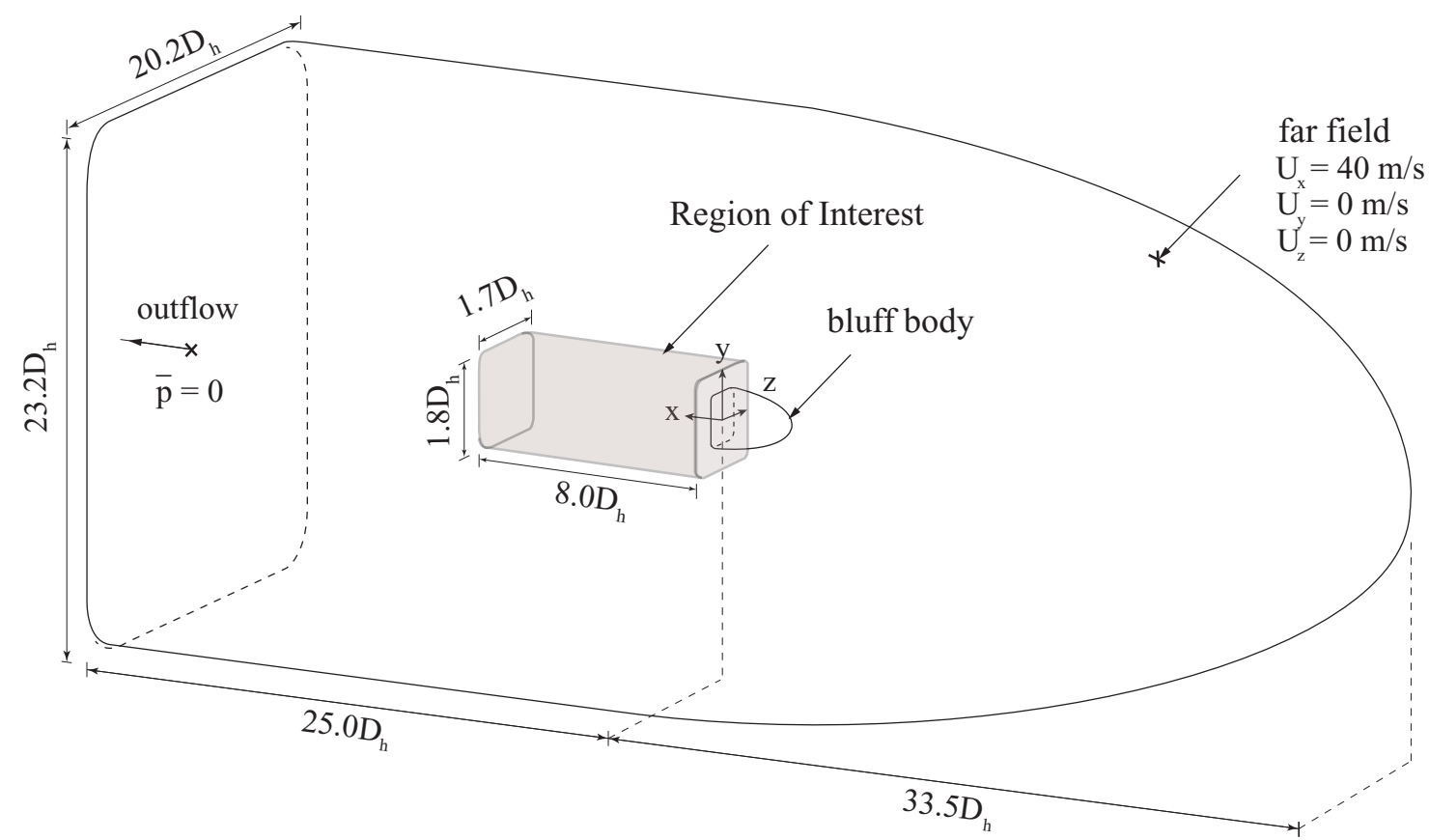

Figure 5.1: Schematic of the computational domain

The Region of Interest is sized to conservatively include all possible transient flow structures associated with the near wake. The surfaces of the bluff-body and its tails 
are set to no-slip walls, and the wall thickness of the tail is set to $1.5 \mathrm{~mm}$, corresponding to $0.8 \%$ of the model's hydraulic diameter, $\mathrm{D}_{\mathrm{h}}$. The placement of the far-field boundary was based on potential flow theory, with the boundary located sufficiently far from the model to yield less than $1 \%$ and $1 \mathrm{deg}$. deviation from the far-field values of the velocity magnitude and direction, respectively. The outflow boundary was placed $25 \mathrm{D}_{\mathrm{h}}$ downstream of the trailing edge of the model without the tail. This is sufficiently far from the model to allow for natural development of the near wake of the model. The far-field velocity magnitude was set to a value that corresponds to a flow Reynolds number value of $5.3 \times 10^{5}$ based on the hydraulic diameter of the model. The values of turbulence kinetic energy, $k$, and specific turbulence dissipation rate, $\omega$, normalized by the far-field velocity and hydraulic diameter of the model, are respectively set to $3.75 \times 10^{-5}$ and 0.663 at the far-field boundary. The velocity flow field in the domain is initialized using the potential flow solution. The turbulence parameters $k$ and $\omega$ are initialized to the far-field values that correspond to a turbulence intensity of $0.5 \%$ and an integral length scale value of $0.1 \mathrm{D}_{\mathrm{h}}$.

At the outflow boundary, the static pressure is kept fixed in an area-averaged sense at the far-field value (0 $\mathrm{Pa}$, gauge).

\subsubsection{Computational Grid}

A spatial grid was mapped to the computational domain, using a combination of an unstructured grid based on tetrahedral cells and a structured grid based on hexahedral cells, as shown in Figure 5.2, Figure 5.3, and Figure 5.4 for the baseline, straighttail and lobed-tail cases, respectively. Table 5.1 provides a summary of the relevant parameters of the grids for the three configurations investigated computationally while the grid nomenclature is presented in Figure 5.5. The aspect ratio and skewness of the grid cells should be kept within certain bounds to prevent excessive build-up of roundoff errors that may hinder the rate of convergence of the iterative solution algorithm. Furthermore, the skewness and the spatial rate of change of the grid-cell size should be kept low to minimize the discretization error in the converged solution. The ranges 
given for these parameters in the table are consistent with the best practices presented in published literature $[141,142]$. Along the walls, the grid cells are clustered using inflation layers containing prism elements to resolve the boundary layer. The wallnormal extent of the flow region covered by the inflation layers was confirmed to include the full thickness of the local boundary layer. The number of inflation layers residing in the boundary layer varies from 9 near the nose of the bluff body to 21 at the trailing edge. Hexahedral cells are used in the Region of Interest and they are clustered within the regions where spatial gradients in velocity are anticipated to be relatively higher. The distance for the first grid node off the wall, $y_{1}^{+}$, is selected such that it remains within the range required by the low-Reynolds-number turbulence model used in the present simulations. Here, the + superscript denotes normalization with the viscous length scale, $\nu / u_{\tau}$, where $\nu$ is the kinematic viscosity, $u_{\tau}=\sqrt{\tau_{w} / \rho}$ is the friction velocity, $\tau_{w}$ is the local wall shear stress calculated at the trailing edge of the bluff-body model, and $\rho$ is the fluid density.

a)

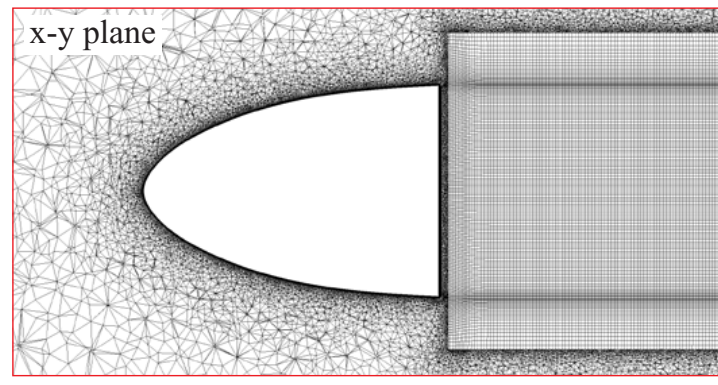

b)

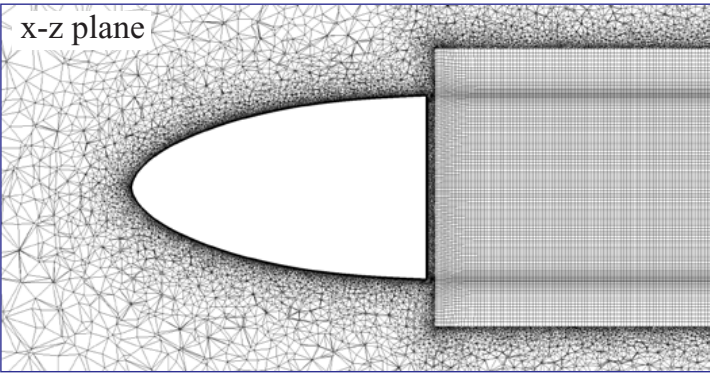

Region of Interest (hexahedral cells)

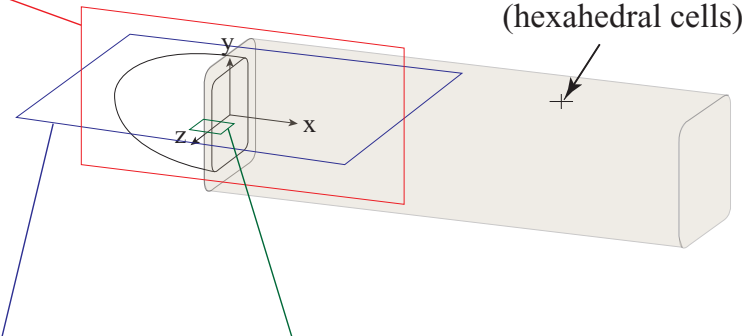

c)

\section{T: prism}

T: tetrahedral

$\mathrm{H}$ : hexahedral

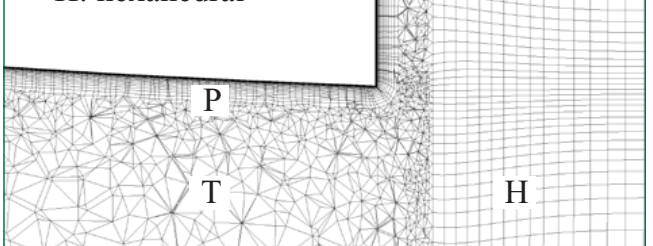

Figure 5.2: Spatial grid for the baseline case 
a)

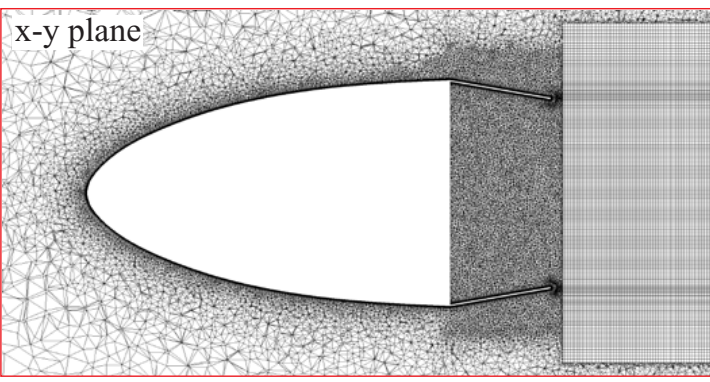

b)
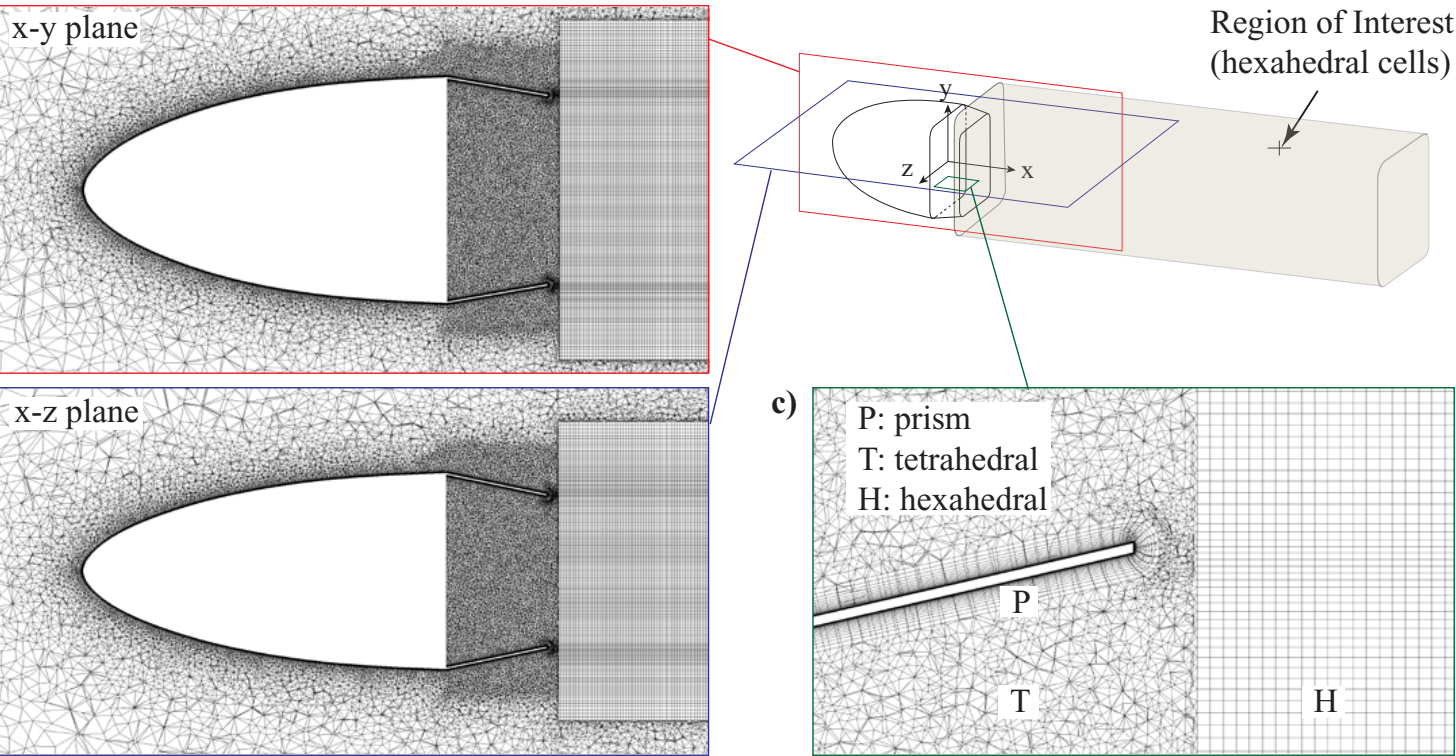

c)

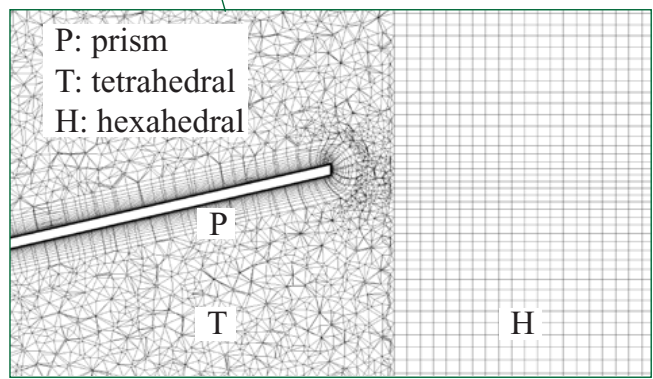

Figure 5.3: Spatial grid for the straight-tail case

a)

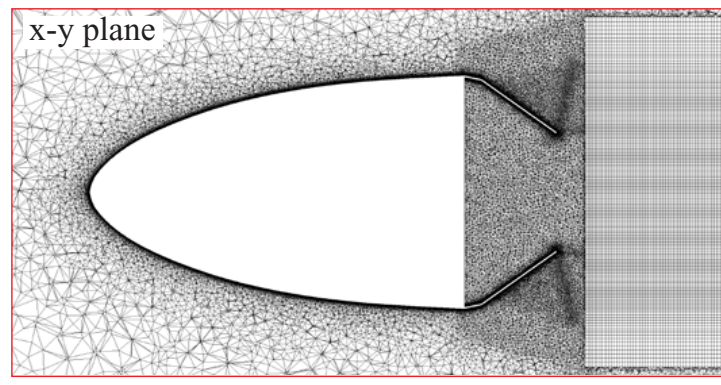

b)
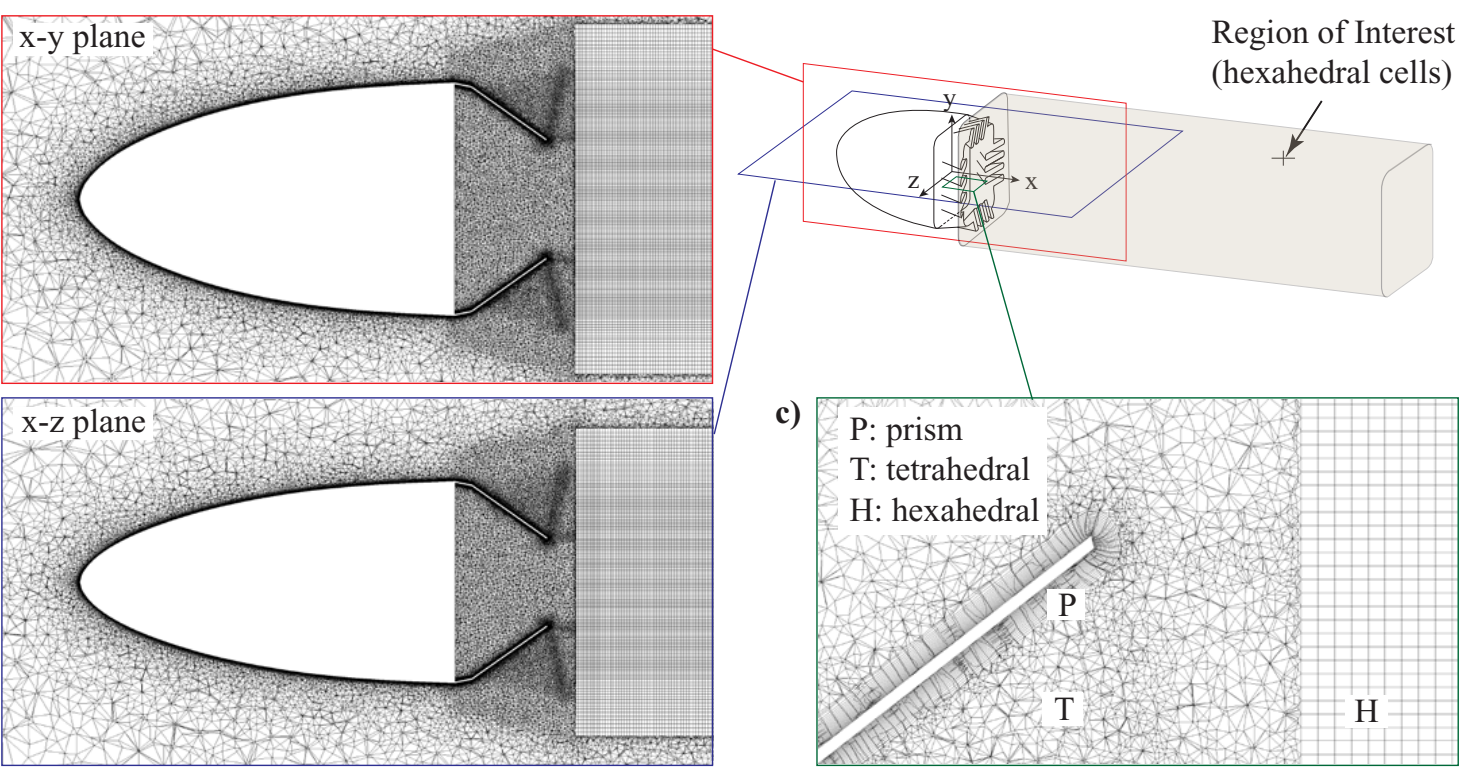

c)

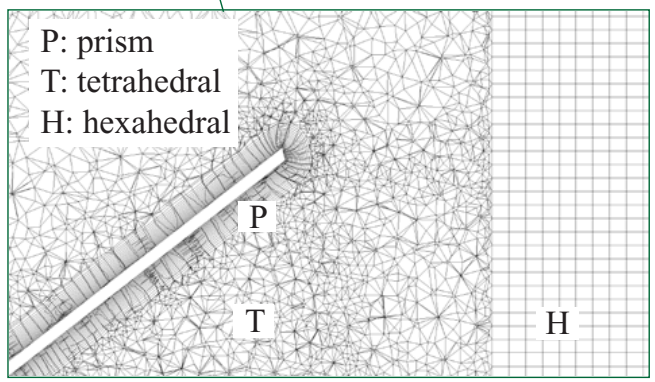

Figure 5.4: Spatial grid for the lobed-tail case

The cell counts of the grids were established through a grid sensitivity analysis, with the computed flow field deemed grid independent when the change in the local velocity vector magnitude and direction anywhere in the domain remained below $1 \%$ upon simultaneous doubling of the grid cell count in each of the three coordinate directions in the wake and vicinity of the bluff-body. 
Table 5.1: Grid specifications

\begin{tabular}{|c|c|c|c|}
\hline Tail Case & Baseline & Straight & Lobed \\
\hline$y_{1}^{+}$ & 0.8 & 0.8 & 0.8 \\
\hline Number of inflation layers & 26 & 26 & 26 \\
\hline Prism max aspect ratio & 225 & 235 & 234 \\
\hline Prism max rate of change of cell size & $15 \%$ & $15 \%$ & $15 \%$ \\
\hline Tetrahedral max aspect ratio & 8 & 9 & 14 \\
\hline Tetrahedral max rate of change of cell size & $14 \%$ & $18 \%$ & $17 \%$ \\
\hline Tetrahedral cell-skewness range & $0-0.5$ & $0-0.5$ & $0-0.5$ \\
\hline Hexahedral max aspect ratio & 5 & 5 & 5 \\
\hline Hexahedral max rate of change of cell size & $15 \%$ & $15 \%$ & $15 \%$ \\
\hline Number of prism elements & $3.8 \times 10^{6}$ & $5.1 \times 10^{6}$ & $12.8 \times 10^{6}$ \\
\hline Number of tetrahedral elements & $8.3 \times 10^{6}$ & $9.9 \times 10^{6}$ & $10.9 \times 10^{6}$ \\
\hline Number of hexahedral elements & $10.6 \times 10^{6}$ & $11.4 \times 10^{6}$ & $10.1 \times 10^{6}$ \\
\hline Total number of grid cells & $23.0 \times 10^{6}$ & $27.8 \times 10^{6}$ & $34.2 \times 10^{6}$ \\
\hline Hexahedral cell & \multicolumn{3}{|c|}{ Cell Aspect Ratio $=\frac{\mathrm{L}_{\max }}{\mathrm{L}_{\min }}$} \\
\hline $\mathrm{L}_{\min }$ & \multirow{2}{*}{\multicolumn{3}{|c|}{$\begin{array}{l}\text { Tetrahedral Cell Skewness }=\frac{R_{2}-R_{1}}{R_{2}} \\
R_{1}=\text { circumradius of the actual cell } \\
R_{2}=\text { circumradius of the equilateral cell }\end{array}$}} \\
\hline l cell & & & \\
\hline
\end{tabular}

Figure 5.5: Grid-cell nomenclature

A time-step size of $2.5 \times 10^{-5} \mathrm{~s}$ was chosen based on the anticipated transient motion of the large-scale flow structures in the wake of the bluff body model. This time-step size corresponds to a Courant number $(C=U \Delta t / \Delta x)$ of 0.45 based on the undisturbed far-field velocity and the average cell size of the spatial grid.

\subsection{Computational Method}

\subsubsection{Governing Equations and Turbulence Modeling}

A commercial computational fluid dynamics (CFD) software, ANSYS-CFX (version 17), was used to solve the incompressible form of the time-varying Reynoldsaveraged Navier-Stokes (RANS) equations: 


$$
\begin{gathered}
\frac{\partial U_{i}}{\partial x_{i}}=0 \\
\frac{\partial U_{i}}{\partial t}+\frac{\partial\left(U_{i} U_{j}+\overline{u_{i}^{\prime} u_{j}^{\prime}}\right)}{\partial x_{j}}=-\frac{1}{\rho} \frac{\partial p}{\partial x_{i}}+\nu \frac{\partial}{\partial x_{j}}\left[\frac{\partial U_{i}}{\partial x_{j}}+\frac{\partial U_{j}}{\partial x_{i}}\right]
\end{gathered}
$$

where $U_{i}$ is the velocity component in the $x_{i}$ Cartesian coordinate direction averaged over a time period that is long compared to the time scale of turbulence, but short in comparison to the time scale of the flow unsteadiness associated with larger transient flow structures. $u_{i}^{\prime}$ is the fluctuating velocity component in the $x_{i}$ Cartesian coordinate direction, $\rho$ is the fluid density, $p$ is the time-averaged static pressure, and $\nu$ is the molecular kinematic viscosity. The over-bar used in the $\overline{u_{i}^{\prime} u_{j}^{\prime}}$ term indicates time averaging.

The Reynolds-stress term, $\overline{u_{i}^{\prime} u_{j}^{\prime}}$, is quantified through the $k-\omega$ shear-stress transport (SST) turbulence model of Menter [143]. The turbulence kinetic energy, $k$, and the specific turbulence dissipation rate, $\omega$, computed via this turbulence model are related to the Reynolds-stress tensor by invoking Boussinesq's hypothesis. In this approach, the Reynolds stresses are assumed to be proportional to the local strain rate, with the eddy viscosity, $\nu_{t}$, serving as the scaling factor. $\nu_{t}$ is related to $k$ and $\omega$ through:

$$
\nu_{t}=\frac{a_{1} k}{\max \left(a_{1} \omega, S_{t} F_{2}\right)}
$$

where $a_{1}$ is a modeling constant set to $0.31, S_{t}$ is the magnitude of the local strain rate, and $\mathrm{F}_{2}$ is a blending function that is 1.0 in the boundary layer and 0.0 elsewhere. In the SST turbulence model, near solid walls the turbulence is computed based on the $k-\omega$ formulation; elsewhere, the $k-\epsilon$ formulation is used. The turbulence dissipation rate, $\epsilon$, is related to $\omega$ through:

$$
\omega=\frac{\epsilon}{C_{\mu} k}
$$


where $C_{\mu}$ is a modeling constant set to 0.09 . The turbulence transport equations for $k$ and $\omega$ in the SST turbulence model are as follows:

$$
\begin{gathered}
\frac{\partial k}{\partial t}+U_{j} \frac{\partial k}{\partial x_{j}}=P_{k}-C_{\mu} \omega+\frac{\partial}{\partial x_{j}}\left[\left(\nu+\sigma_{k} \nu_{t}\right) \frac{\partial k}{\partial x_{j}}\right] \\
\frac{\partial \omega}{\partial t}+U_{j} \frac{\partial \omega}{\partial x_{j}}=\alpha S_{t}^{2}-\beta \omega^{2}+\frac{\partial}{\partial x_{j}}\left[\left(\nu+\sigma_{\omega} \nu_{t}\right) \frac{\partial \omega}{\partial x_{j}}\right]+2\left(1-F_{1}\right) \sigma_{\omega 2} \frac{1}{\omega} \frac{\partial k}{\partial x_{i}} \frac{\partial \omega}{\partial x_{i}}
\end{gathered}
$$

where $F_{1}$ is a blending function, and $P_{k}$ is the rate of production of turbulence kinetic energy that is computed using:

$$
P_{k}=\left[\nu_{t}\left(\frac{\partial U_{i}}{\partial x_{j}}+\frac{\partial U_{j}}{\partial x_{i}}\right)-\frac{2}{3} k \delta_{i}\right] \frac{\partial U_{i}}{\partial x_{j}}
$$

where $\delta_{i}$ is the Kronecker delta. The empirical modeling constants used in Eqs. 5.3-5.6, as proposed by Menter and Esch [144], are tabulated in Table 5.2.

Table 5.2: Closure constants in the SST turbulence model

\begin{tabular}{cc}
$k-\omega$ & $k-\epsilon$ \\
\hline$\alpha_{1}=5 / 9$ & $\alpha_{2}=0.44$ \\
$\sigma_{k 1}=0.85$ & $\sigma_{k 2}=1.0$ \\
$\sigma_{\omega 1}=0.5$ & $\sigma_{\omega 2}=0.856$ \\
$\beta_{1}=0.075$ & $\beta_{2}=0.0828$ \\
\hline
\end{tabular}

Given the possibility that along the walls of the buff-body tails, the turbulence in the wall boundary layers may deviate from equilibrium state, and the boundary layer may experience separation in certain regions, the turbulence equations are integrated to the wall rather than relying on wall functions. At the no-slip surface, the values of $k$ and $\nu_{t}$ are set to zero, and the value of $\omega$ is computed using: 


$$
\omega_{\text {wall }}=10 \frac{6 \nu}{\beta_{1} \mathrm{y}_{1}^{2}}
$$

where $y_{1}$ is the distance for the first grid node off the wall. Assuming isotropic turbulence during the initialization of the flow domain, the value of $k$ is estimated by:

$$
k=\frac{3}{2}\left(I\left|U_{r e f}\right|\right)^{2}
$$

where $I$ is the turbulence intensity, and $U_{r e f}$ is the reference velocity. Similarly, the value of $\omega$ is estimated by:

$$
\omega=\frac{\sqrt{k}}{C_{\mu} L}
$$

where $L$ is the integral length scale.

\subsubsection{Discretization}

In the solution algorithms utilized by the Ansys CFX software, the mass conservation equation is modified by expressing the velocity appearing in this equation in terms of pressure via the momentum conservation equation, thereby yielding Poisson's equation governing the pressure distribution in the flow field.

The spatial discretization is based on the vertex-centered finite volume technique, with a collocated grid arrangement so that all variable values are stored at the grid nodes. Pressure-velocity coupling is achieved in the scheme of Rhie and Chow [145], whereby the velocities at the integration points are expressed as an average of the surrounding nodal values adjusted by a redistribution of pressure gradients at the faces of the finite volume. The non-linear convention term in the momentum equations are linearized via Picard iteration, which uses the method of successive approximations by computing the momentum components using the velocity from the current 
iteration and the mass flux from the previous iteration [146]. The spatial interpolations of the flow parameters from grid nodes to the control volume surfaces are realized in manners that are equivalent to second-order centered differencing of all spatial derivatives except for those in the convection term of the momentum equations which are approximated using a hybrid of first-order upwind and second-order centered differencing. The time derivatives are discretized via second-order backward differencing.

\subsubsection{Solution Method}

The linear system of discretized momentum and turbulence transport equations are solved using a multigrid accelerated Incomplete Lower-Upper (ILU) factorization method [147]. The convergence of the solution is accelerated using a W-type Algebraic-Multigrid technique to solve the governing equations on a series of successive coarser grids. Eight grid levels, determined after an initial simulation to optimize the rate of convergence, are used in the restriction and prolongation legs of the W-cycle with three smoothing iterations performed per grid level. The transient solution is obtained with one W-cycle for a fixed set of coefficients of the linearized governing equations, and a sufficient number of updates of these coefficients per time step. In each time step, iterative solution of the discretized governing equations is declared converged when the root-mean-square residuals of the governing equations are reduced to less than $1 \times 10^{-6}$. For the baseline case, long-straight tail case and long-lobed tail case, this is realized with up to ten, seven, and nine coefficient-update iterations per time step, respectively. Approximately, 4500 time steps were required for the flow to reach statistically steady state in each case, after which 2000 additional time steps were computed for analysis. 


\section{Chapter 6}

\section{Results and Discussion}

\subsection{Experimental Results}

Experimental configurations tested in the wind tunnel are shown in Figure 6.1, with the measured drag coefficients tabulated in Table 6.1.

a) Baseline

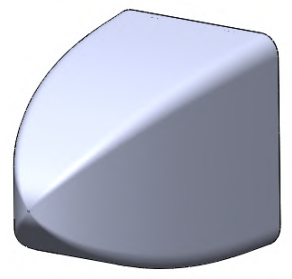

d) Long-Curved Tail

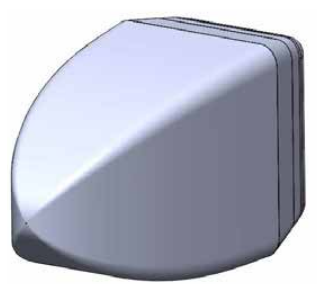

b) Long-Straight

Tail

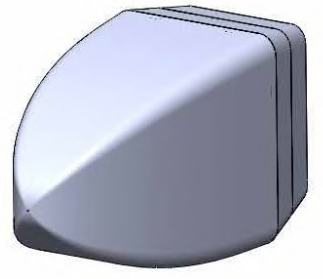

e) Long-Small-Lobed Tail

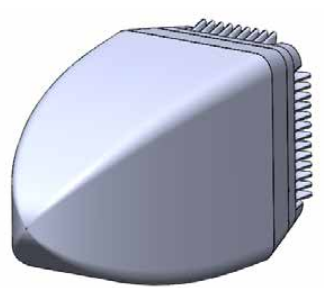

c) Short-Straight

Tail

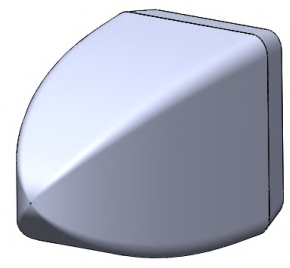

f) Long-Large-Lobed Tail

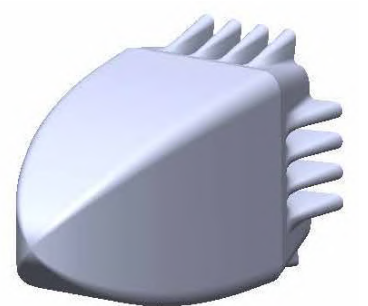

Figure 6.1: Experimental tail configurations

Table 6.1: Measured drag coefficients

\begin{tabular}{lc}
\hline Configuration & $C_{D}$ \\
\hline Baseline & 0.26 \\
Long-Straight Tail & 0.12 \\
Short-Straight Tail & 0.20 \\
Long-Curved Tail & 0.18 \\
Long-Small-Lobed Tail & 0.21 \\
Long-Large-Lobed Tail & 0.27 \\
\hline
\end{tabular}


Amongst the tail configurations tested, the long-straight tail appears to be most effective with about $55 \%$ reduction in drag compared to that of the baseline case. Reducing the tail length appears to have an adverse effect on the boat-tail performance, similar to what has been observed in the literature [103,148]. Interestingly, incorporating a short inwardly-curved segment to the long straight tail to promote increase of the base pressure by directing the flow towards the wake core appears instead to reduce the effectiveness of the tail. This may be due to the chosen radius for the curved segment of the tail. A relatively sharp inward turn of the tail surface would be too difficult for the boundary layer flow to follow, prompting separation of the boundary layer upstream of the trailing edge. Such separation would result in reduced inward flow in the wake compared to the straight configuration, explaining the observed drag trend between the two configurations. The presence of lobes on the tail, at least for the specific lobe geometries that are considered in this study, appears to partially or completely offset the the drag-reduction benefit of a straight tail. The identification of the flow physics behind this drag trend that occurs despite the anticipated enhancement in cross-stream mixing driven by the lobed trailing edge geometry requires investigation of the wake dynamics for these configurations. Such an investigation is warranted, for despite the apparent drag penalty associated with lobed tails, such tails may yield a wake with transient vortical structures of smaller length and/or time scales. Reducing the size/strength of the transient flow structures in the wake may be desirable in certain applications, such as the consideration of wake hazard for following traffic in road transportation. Studies documenting the wake dynamics in the vicinity of the tail region are needed to identify tail configurations that are more effective in such contexts. The computational results presented in the next section compare the wake dynamics of the baseline, long-straight tail and long-large-lobed tail configurations to inform future design optimization efforts. 


\subsection{Computational Results}

\subsubsection{Baseline Configuration}

The vortical structures developing in the near-wake of the streamlined bluff body for the baseline geometry are visualized through isosurfaces of the second invariant of the velocity gradient tensor, Q, in Figure 6.2. The Q parameter is expressed as:

$$
Q=\frac{1}{2}\left(\frac{\partial U_{i}}{\partial x_{i}} \frac{\partial U_{j}}{\partial x_{j}}-\frac{\partial U_{i}}{\partial x_{j}} \frac{\partial U_{j}}{\partial x_{i}}\right)
$$

where $U_{i}$ and $U_{j}$ represent the components of the velocity vector, with Einstein summation applied over the indexed terms. The value of $\mathrm{Q}$ at which the iso-surfaces are plotted is chosen to most clearly show the vortical structures which are deemed to potentially have a notable effect on the dynamics of the wake. The isosurfaces are coloured by the normalized streamwise velocity, $U_{x} / U_{\text {ref }}$. These results are obtained for nominally laminar conditions, realized by turning off the turbulence model in the computational model. On the larger scale, the bluff-body wake is dominated by a streamwise-aligned helical pattern that is remarkably similar to the wake of a sphere. On the basis of this similarity, it may be argued that this helical pattern is the result

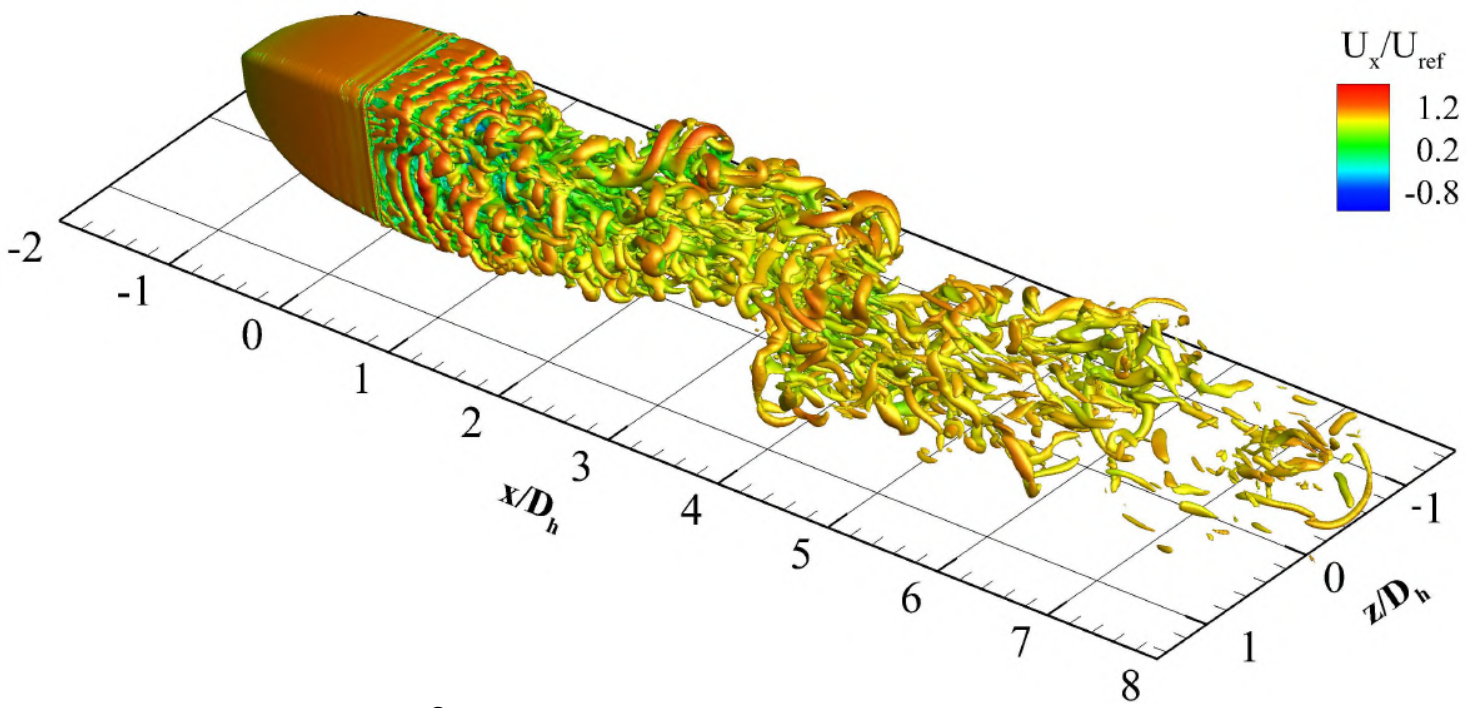

Figure 6.2: $\mathrm{Q} /\left(\mathrm{U}_{\text {ref }} / \mathrm{D}_{\mathrm{h}}\right)^{2}=9.5$ isosurfaces for the baseline configuration in the absence of small-scale turbulence 
of the same mechanism as that observed for spheres, namely a local absolute instability which develops just upstream of the rear stagnation point of the recirculation zone located at $\mathrm{x} / \mathrm{D}_{\mathrm{h}}=1.4$. The pitch of the helical pattern in Figure 6.2 corresponds to an orbit speed of the local absolute instability that is equal to $1.4 \mathrm{U}_{\text {ref }} / \mathrm{D}_{\mathrm{h}}$. This angular speed is noted to be consistent with the observations in the wake of spheres [43]. Even though the sense of rotation of the helical pattern remained the same throughout the length of the present simulations, it is quite plausible that this could alternate between clockwise and counter-clockwise directions randomly over longer time periods, similar to what has been observed in the wake of axisymmetric bluff bodies [31,43]. The aspect ratio of the rectangular base $(\mathrm{AR}=1.15)$ falls within the range for which reflectional symmetry breaking (RSB) instability has been observed in the published literature for rectilinear bodies similar to the Ahmed body as was reviewed in Chapter 3. It is therefore interesting to note the absence of the RSB mode in the manner it has been observed in the wake of box-shaped bodies with a rectangular base. This may suggest that the development of the RSB mode is affected by not only the aspect ratio of the base, but also the shape of the body affecting the streamwise development of the boundary layer along the body surface starting from the leading-edge stagnation point, and the resultant circumferential variation of the characteristics of the boundary layer along the perimeter of the base.

In close vicinity of the base (Figure 6.3), the boundary layer separating along the base perimeter is observed to form a train of vortex rings, which form due to the well-known Kelvin-Helmholtz inviscid instability mode triggered by the inflectional velocity profile of the shear layer immediately downstream of the location of separation. The streamwise spacing of the vortex rings yields a vortex shedding frequency that corresponds to a Strouhal number of 6.0. This Strouhal number value is in favourable agreement with published results in the near wake of bluff bodies [43]. The vortex rings are noted to disintegrate a short distance from the base due to secondary and tertiary instabilities as has been well-documented for free shear layers in the published literature $[40,121]$. As a result, only about 4 to 5 intact vortex rings are observed in the train of vortex rings at any given instant in time. 


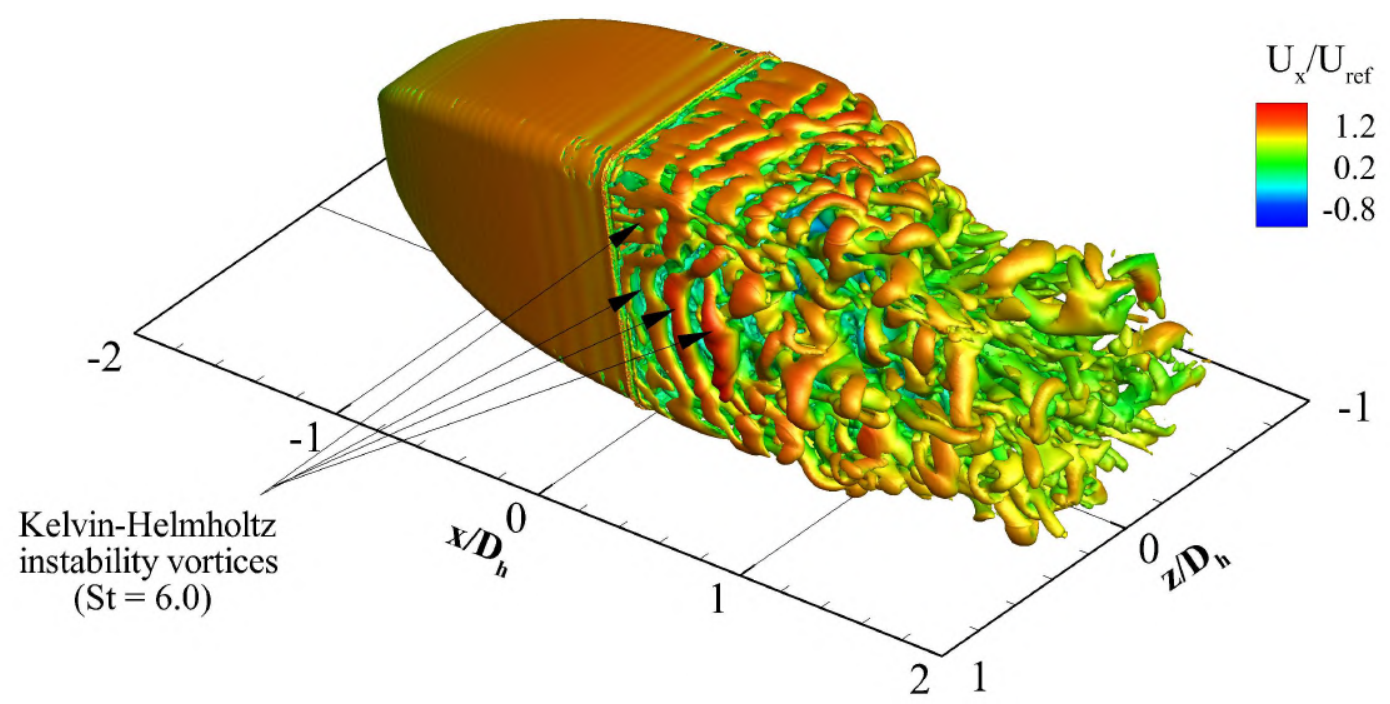

Figure 6.3: $\mathrm{Q} /\left(\mathrm{U}_{\text {ref }} / \mathrm{D}_{\mathrm{h}}\right)^{2}=9.5$ isosurfaces in the near wake for the baseline configuration in the absence of small-scale turbulence

The physics observed through the Q plots in Figures 6.2 and 6.3 and discussed in the foregoing are schematically contrasted to the published observations of the wake of spheres in Figure 6.4.

The effect of small-scale turbulence, accounted for in the simulations via a turbulence model, is evident by comparing the $\mathrm{Q}$ plots in Figure 6.5 with those in Figure 6.2. It should be noted that the isosurface $Q$ value in Figure 6.5 is three orders of magnitude smaller than that in Figure 6.2. This comparison demonstrates the substantial weakening of the larger scale coherent vortical structures in the wake region by the diffusive and dissipative effects of small-scale turbulence. The periodic shedding of vorticity rings off the base perimeter and the development of a helical pattern driven by a local instability that are observed in the absence of small-scale turbulence are noted to be suppressed by small-scale turbulence. The only dominant feature in the wake region is noted to be a steady recirculation zone residing within the first hydraulic diameter streamwise distance of the base. Farther downstream of the wake, there is some evidence of coherent vortical structures, which may plausibly form through amalgamation of smaller vortical structures. Nonetheless, their strength remains orders of magnitude smaller than the structures residing in the wake at this distance from the body in the absence of small-scale turbulence. 
a)

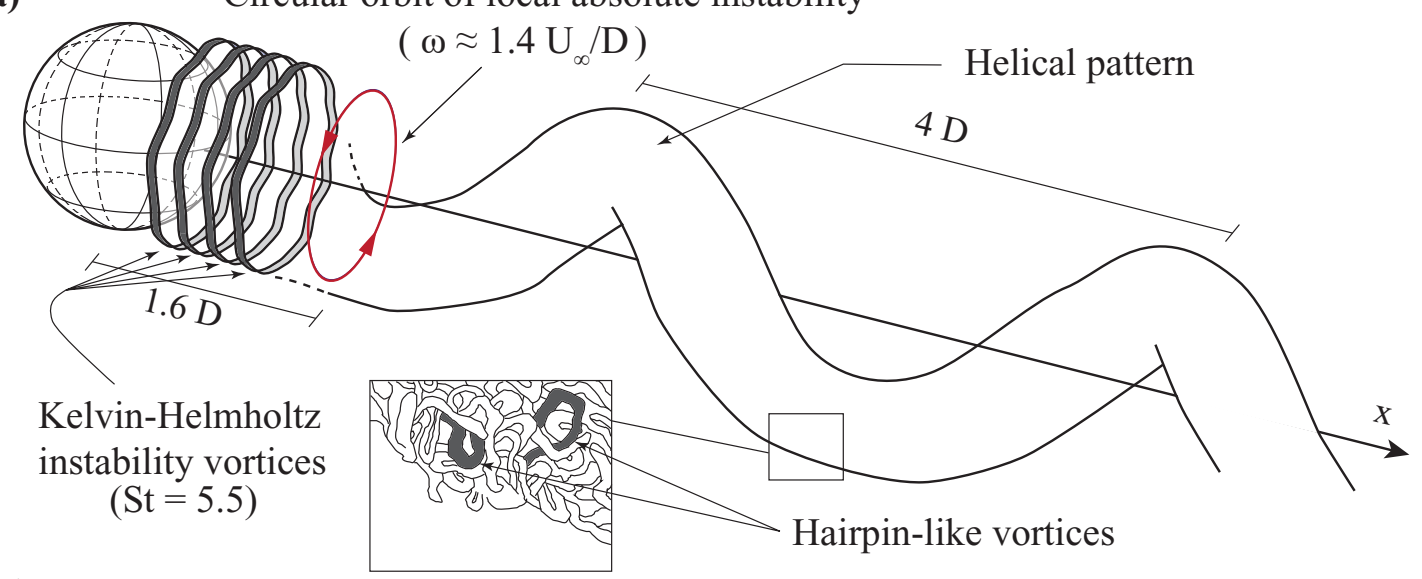

b)

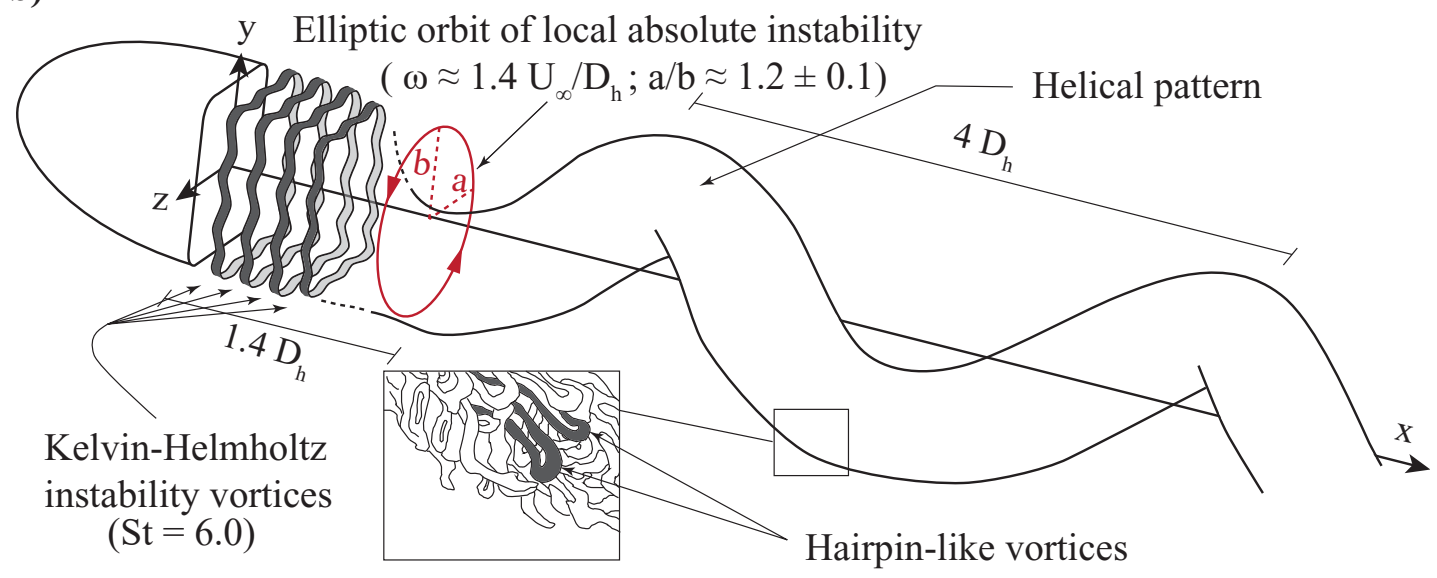

Figure 6.4: Schematic representation of the wake structure for a) the sphere [43], and b) the present bluff body

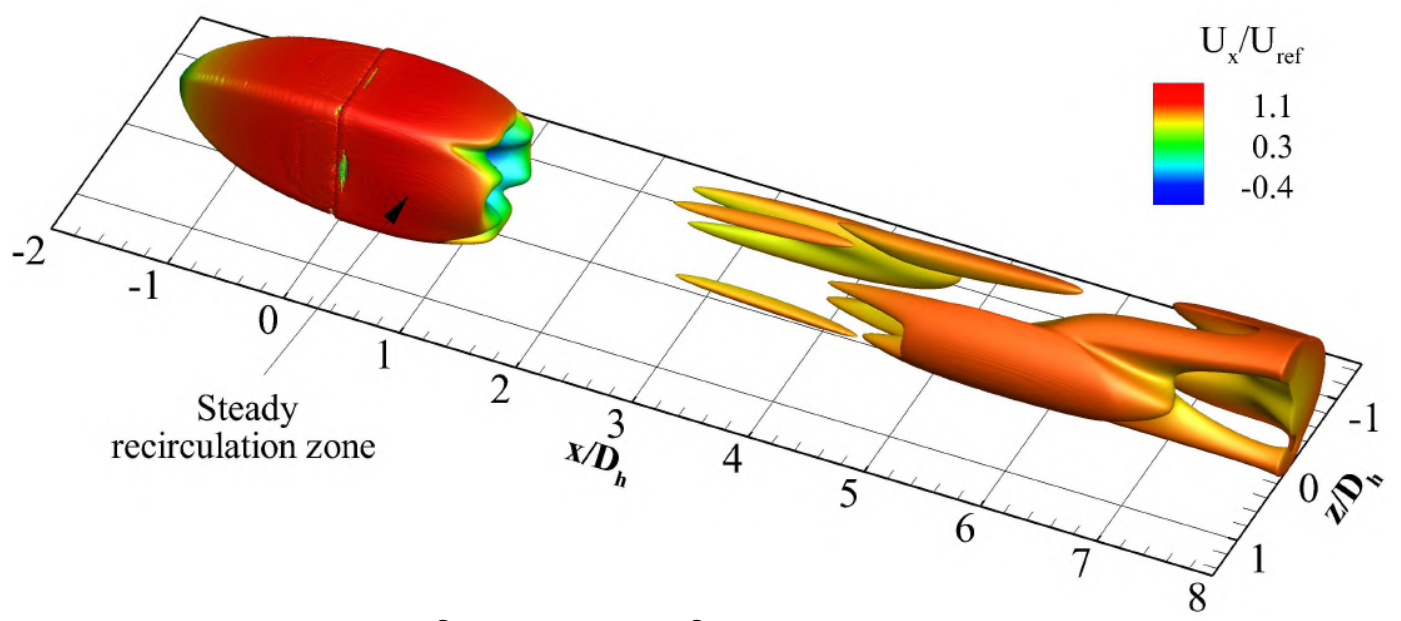

Figure 6.5: $\mathrm{Q} /\left(\mathrm{U}_{\mathrm{ref}} / \mathrm{D}_{\mathrm{h}}\right)^{2}=1.2 \times 10^{-3}$ isosurfaces for the baseline configuration in the presence of small-scale turbulence 
a)

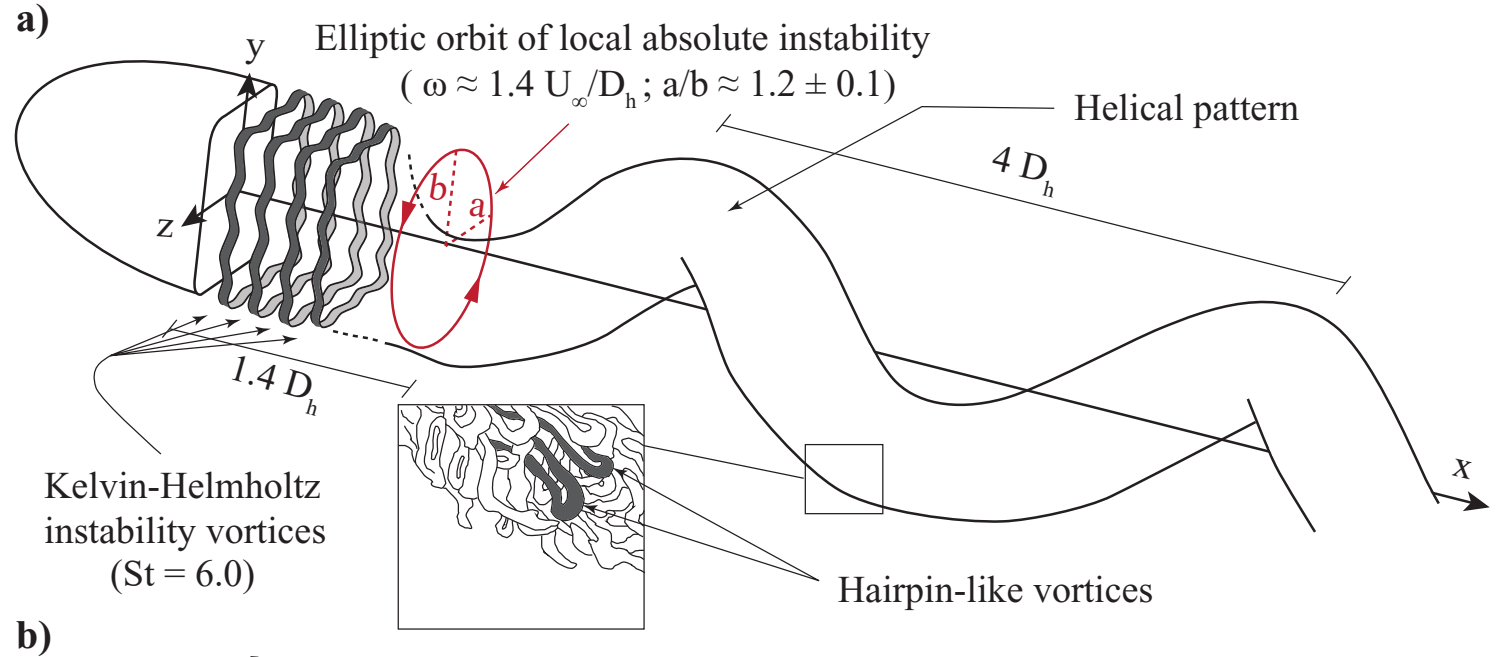

b)

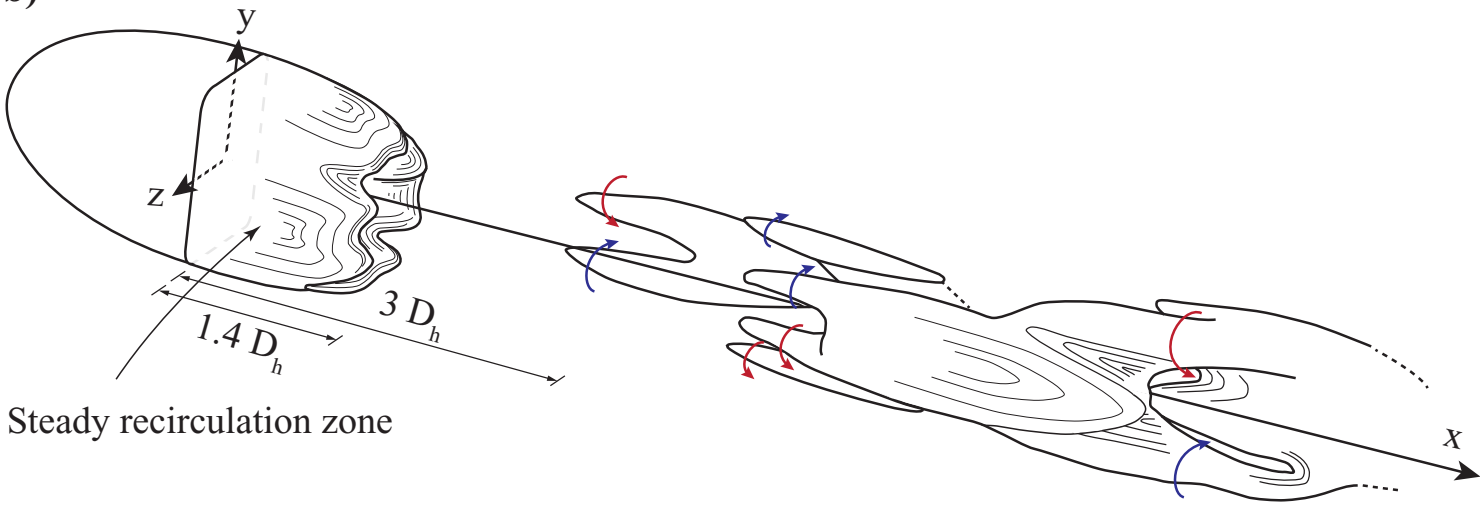

Figure 6.6: Schematic representation of the wake structure a) in the absence of small-scale turbulence, $b$ ) in the presence of small-scale turbulence

The dominant features of the wake in the presence and absence of small-scale turbulence are contrasted schematically in Figure 6.6.

The understanding of bluff-body wake dynamics is most commonly used to inform design optimization of the body to improve its aerodynamic performance. In certain applications, the dynamics of the wake region is of further importance due to the hazard it may pose. The hazard created by the transient aerodynamic forces in the wake region of large transport trucks for smaller following vehicles serves as a good example to this. To investigate the hazard characteristics of the wake of the present bluff body, the wake was divided into several concentric zones, and the wake was analyzed in these zones at several axial locations as shown in Figure 6.7. 
a) Isometric view

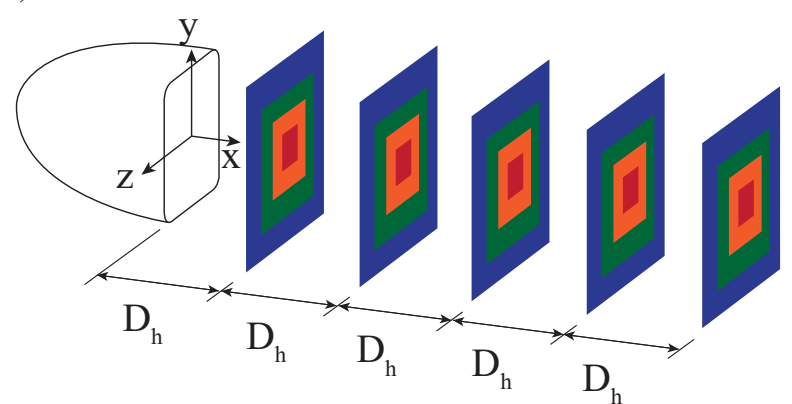

b) Rear view

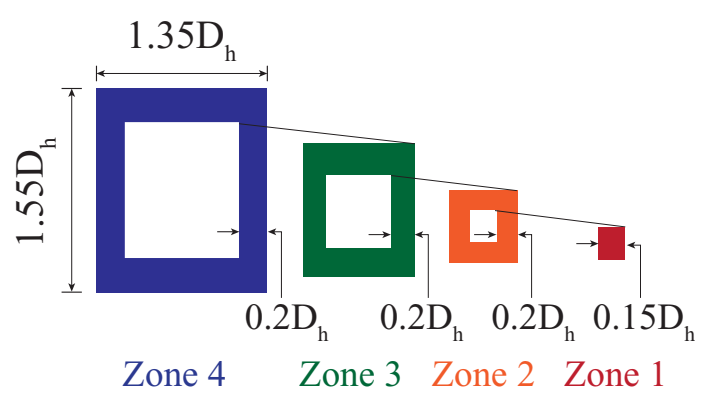

Figure 6.7: Planes of interest in the bluff-body wake

The kinetic energy and length scale of the larger transient structures are two parameters that could be argued to be particularly relevant to the magnitude and duration of transient aerodynamic forces, and hence the hazard such structures create within the wake region. The kinetic energy of the transient structures was computed based on the fluctuating part of the Cartesian velocity components as per the following expression:

$$
k_{L S}=\left(\left(U_{x}-\overline{U_{x}}\right)^{2}+\left(U_{y}-\overline{U_{y}}\right)^{2}+\left(U_{z}-\overline{U_{z}}\right)^{2}\right) / 2
$$

where the LS subscript is used to indicate that this kinetic energy corresponds to the energy of the larger scale transient structures given that in the simulations with the turbulence model turned off, the velocity fluctuations associated with the sub-grid scale turbulence are not captured in the time resolved velocity field. In Eq. 6.2, the overbar denotes time averaging over 2000 time steps, which corresponds to about 100 shedding cycles of the vorticity rings in the case without small-scale turbulence. In each data analysis plane, $k_{L S}$ was arithmetically averaged over 2900 locations uniformly distributed across the four zones.

As is evident in Figure 6.8, in the absence of small-scale turbulence, the kinetic energy of the transient coherent flow structures decreases rapidly with streamwise distance in all zones, with the rate of decease correlating with the magnitude of this energy close to the base of the body. The majority of the energy is noted to be confined to the inner two zones. In contrast, the $k_{L S}$ parameter is essentially zero in the wake of the body when small-scale turbulence is accounted for via the turbulence closure of the computational model. 


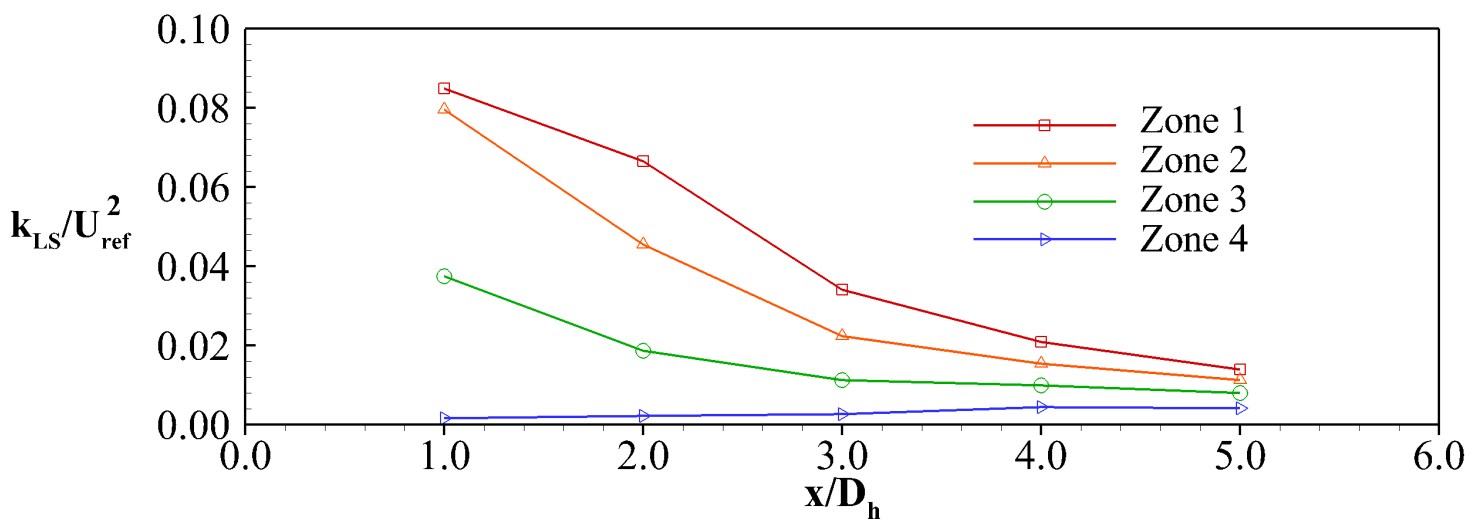

Figure 6.8: Kinetic energy of larger-scale transient structures in the absence of small-scale turbulence for the baseline configuration

The length scale of the larger transient coherent structures was estimated in the absence of small-scale turbulence using Taylor's frozen eddy hypothesis applied to the streamwise component of velocity fluctuations [149]. The distribution of this length scale shown in Figure 6.9 indicates that the larger scale motion is concentrated to the outer zones of the wake, which is expected. Given the concentration of larger length scales in the outer regions of the wake while the higher transient-motion kinetic energy appears to reside in the core of the wake, identification of regions of wake that may be argued to be more hazardous is not straightforward. Such a hazard analysis may be better performed for a specific object situated within the wake by analyzing

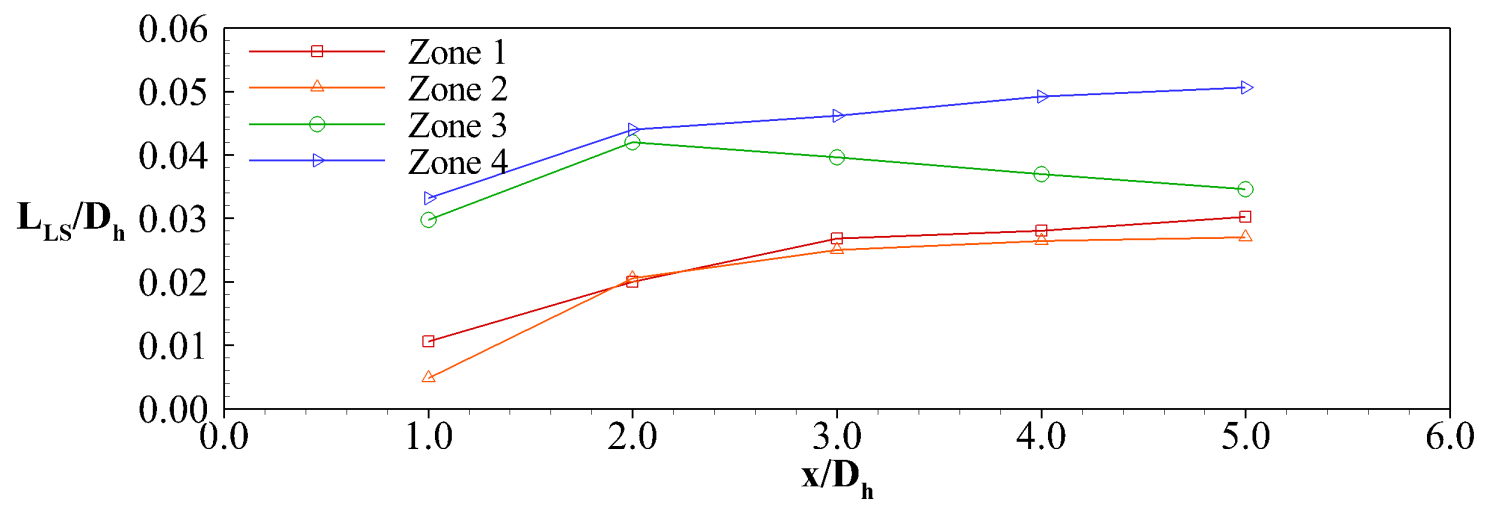

Figure 6.9: Length scale of larger-scale transient structures for the baseline configuration in the absence of small-scale turbulence 
the magnitude and time scale of the resultant transient aerodynamic forces on the object. Such an analysis would also account for the modification of the local wake by the object itself, which falls beyond the scope of the present study.

The cross-stream mixing of the wake due to larger-scale three-dimensional motions described in the foregoing, smaller scale turbulence, and molecular diffusion promotes increased flow uniformity in the wake with streamwise distance. This is illustrated through streamwise variation of axial momentum in the wake zones in Figure 6.10.

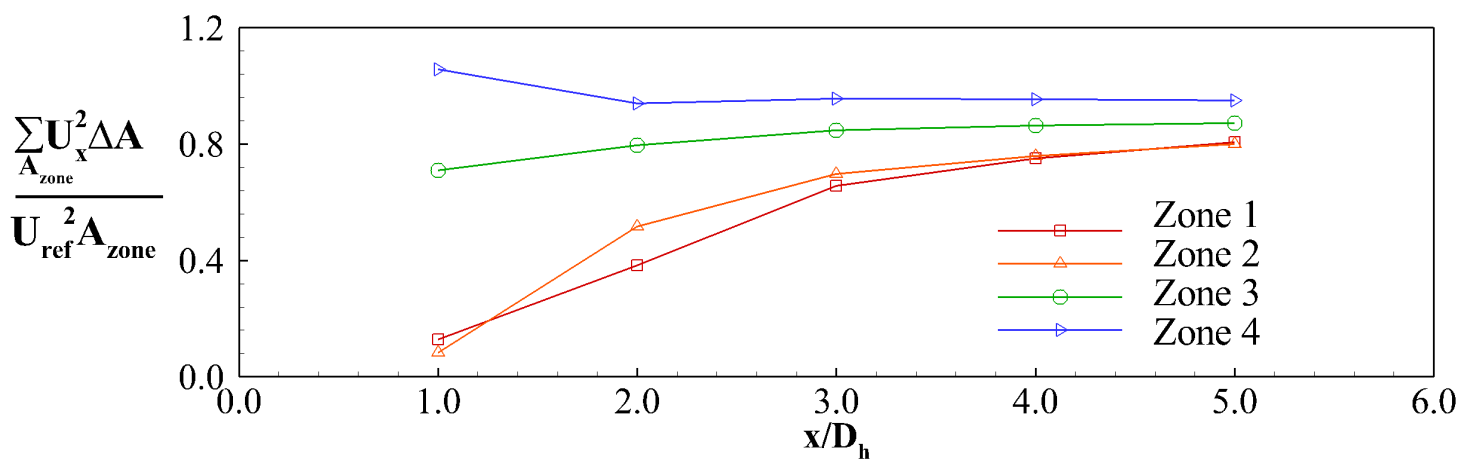

Figure 6.10: Axial momentum flux distribution in the wake for the baseline configuration in the absence of small-scale turbulence

In the absence of small-scale turbulence, the dominant mechanism of mixing is the larger scale transient structures. Via this mechanism, the axial momentum flux in the four zones is observed to gradually converge over the first five hydraulic diameters. The convergence is not complete at this streamwise location, and is anticipated to remain as such for some streamwise distance given the relatively small slopes in the observed trends. For the turbulent case, where any larger scale transient motions of the laminar wake are observed to be suppressed, the smaller scale turbulence is the primary mixing mechanism. The trends in Figure 6.11 indicate that this mechanism is notably less effective than the larger scale motions of the laminar case to promote homogeneity of the wake across all four zones. 


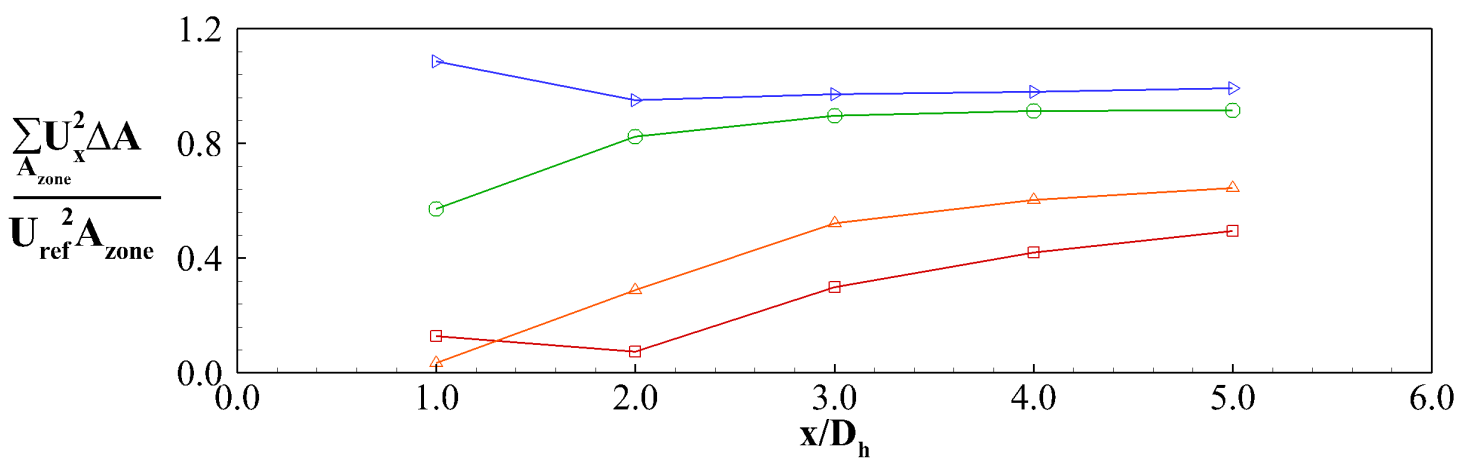

Figure 6.11: Axial momentum flux distribution in the wake for the baseline configuration in the presence of small-scale turbulence

\subsubsection{Straight-Tail Configuration}

The effect of a boat-tail on the wake development is illustrated through isosurfaces of $\mathrm{Q}$ in Figure 6.12. Comparing this flow field to that of the baseline configuration in Figure 6.2, one notes a notable weakening of the helical pattern. This suggests that the local rotational instability in the recirculation zone responsible for the helical wake shape may either be absent or be substantially reduced in strength in the presence of the tails. The boundary layer is separated on the tail surfaces, which yields a highly inflectional surface-normal profile of streamwise velocity, which is inviscid unstable.

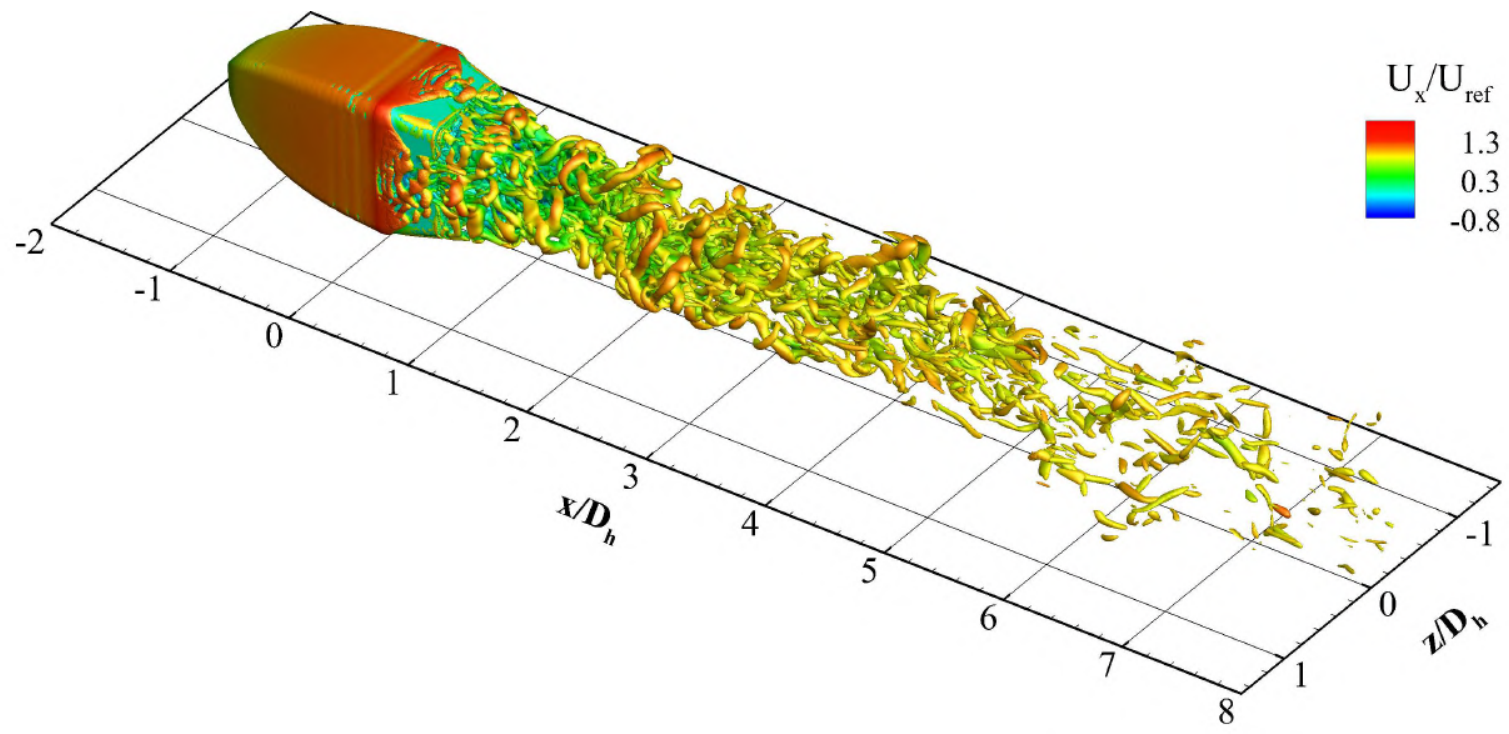

Figure 6.12: $\mathrm{Q} /\left(\mathrm{U}_{\text {ref }} / \mathrm{D}_{\mathrm{h}}\right)^{2}=9.5$ isosurfaces for the straight-tail configuration in the absence of small-scale turbulence 
This instability results in streamwise grouping of spanwise vorticity in the separated shear layer as is visible in Figure 6.13. This fundamental instability process has been thoroughly studied in the context of separation bubbles on airfoils [150]. Following this streamwise grouping, yet another instability promotes waviness of the spanwise vorticity lines, which triggers a self-induction process to form hairpin-like vortices as observed in Figure 6.13. This development is noted to be suppressed at the four rounded corners of the boat tail, plausibly due to differences in the characteristics of the boundary layer at the leading end of the tail at these corners compared to the flat sections. The wake cross section is notably reduced compared to the no-tail case, which is well-known to be the primary effect of boat-tails to realize higher base pressures hence reduced drag on bluff bodies.

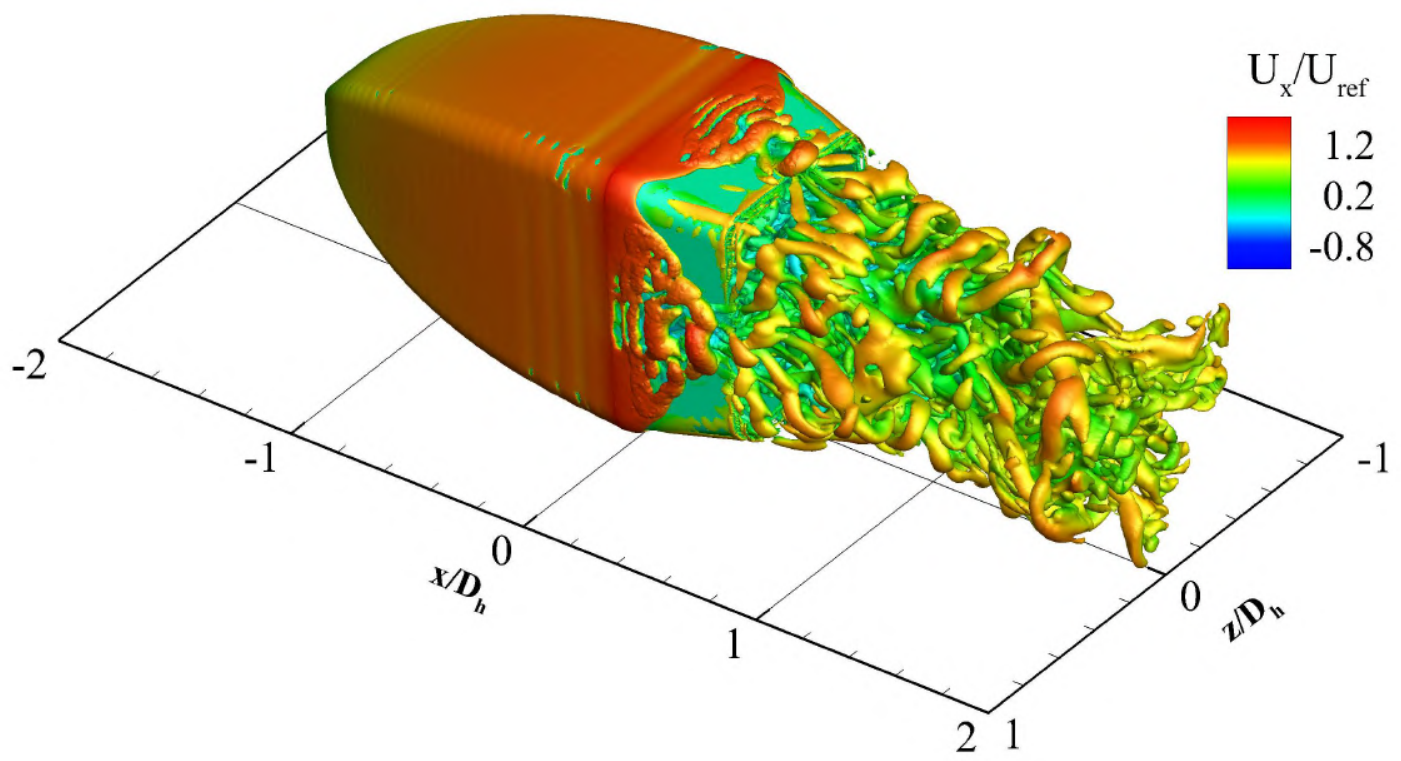

Figure 6.13: $\mathrm{Q} /\left(\mathrm{U}_{\mathrm{ref}} / \mathrm{D}_{\mathrm{h}}\right)^{2}=9.5$ isosurfaces in the near wake for the straight-tail configuration in the absence of small-scale turbulence

The effect of small-scale turbulence on the wake flow field shown in Figure 6.14 is somewhat similar to what was noted for the baseline configuration (Figure 6.5). The boundary layer separation on the tail surfaces is not as severe as for the laminar case, hence the aforementioned instability-driven streamwise-periodic flow development is suppressed. The near wake develops into a steady recirculation zone, and weak largerscale vortical structures prevail farther downstream. The vortical structures appear 


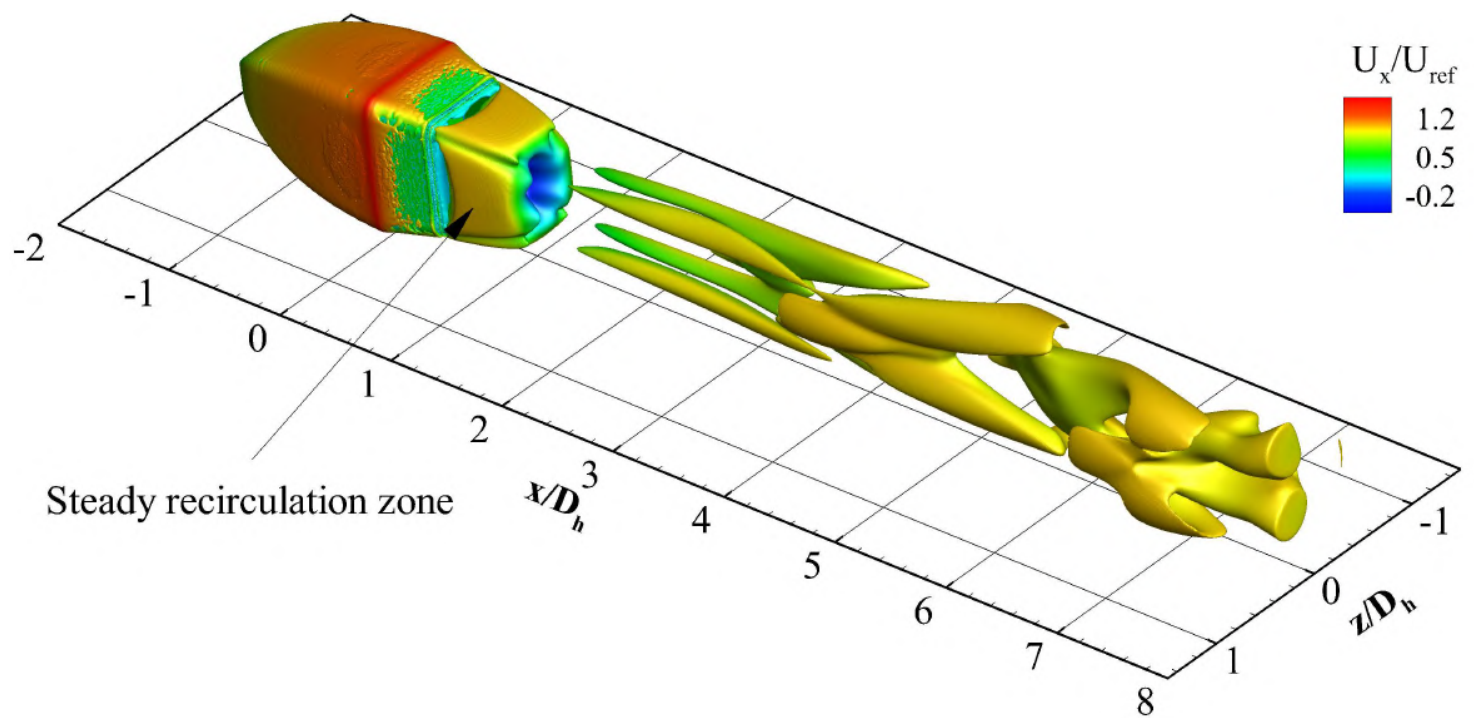

Figure 6.14: $\mathrm{Q} /\left(\mathrm{U}_{\mathrm{ref}} / \mathrm{D}_{\mathrm{h}}\right)^{2}=2.4 \times 10^{-2}$ isosurfaces for the straight-tail configuration in the presence of small-scale turbulence

in pairs, which may suggest that they exist as hairpin-like vortices only parts of which are visible in this plotting format. The streamwise spacing of these pairs appears to be periodic, corresponding to a Strouhal number of 0.7. This streamwise periodic formation of coherent vortical structures is similar to the observations in the wakes of spheres discussed in Chapter 3 (Figure 3.1).

The spatial distribution of the kinetic energy of the transient flow structures for the case without small-scale turbulence is shown in Figure 6.15. Comparing this distribution to that of the baseline configuration (Figure 6.8), the energy in the first

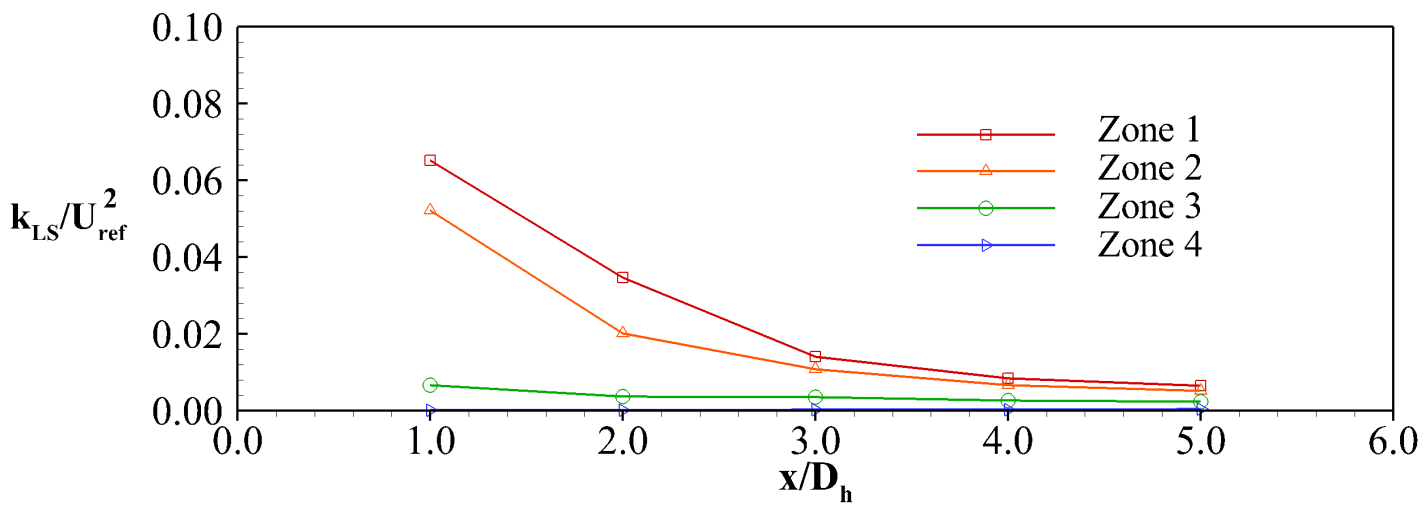

Figure 6.15: Kinetic energy of larger-scale transient structures in the absence of small-scale turbulence for the straight-tail configuration 
three (inner) zones is observed to be notably lower in the presence of a tail. Furthermore, the streamwise rate of decrease of this energy in the core zones where the energy is highest is observed to be significantly higher for the tailed configuration. The corresponding length scales shown in Figure 6.16 have similar magnitudes and trends as for the baseline configuration (Figure 6.9).

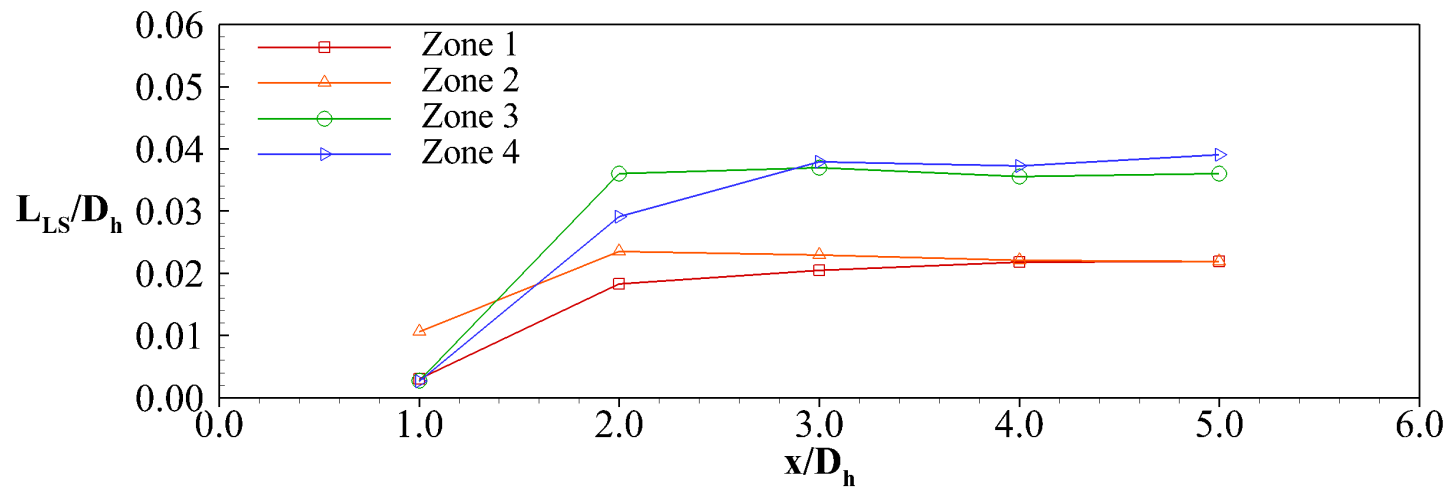

Figure 6.16: Length scale of larger-scale transient structures for the straight-tail configuration in the absence of small-scale turbulence

The mixing of the wake with streamwise distance described through streamwise variation of axial momentum in the four wake zones in Figure 6.17 suggest that the mixing is more rapid in the near wake, with the axial momentum of zones 1 and 2 increasing notably faster than for the baseline configuration. This trend is consistent with the above-noted more rapid streamwise decrease in the transient kinetic energy in these zones for the tailed configuration. The effect of small-scale turbulence on the

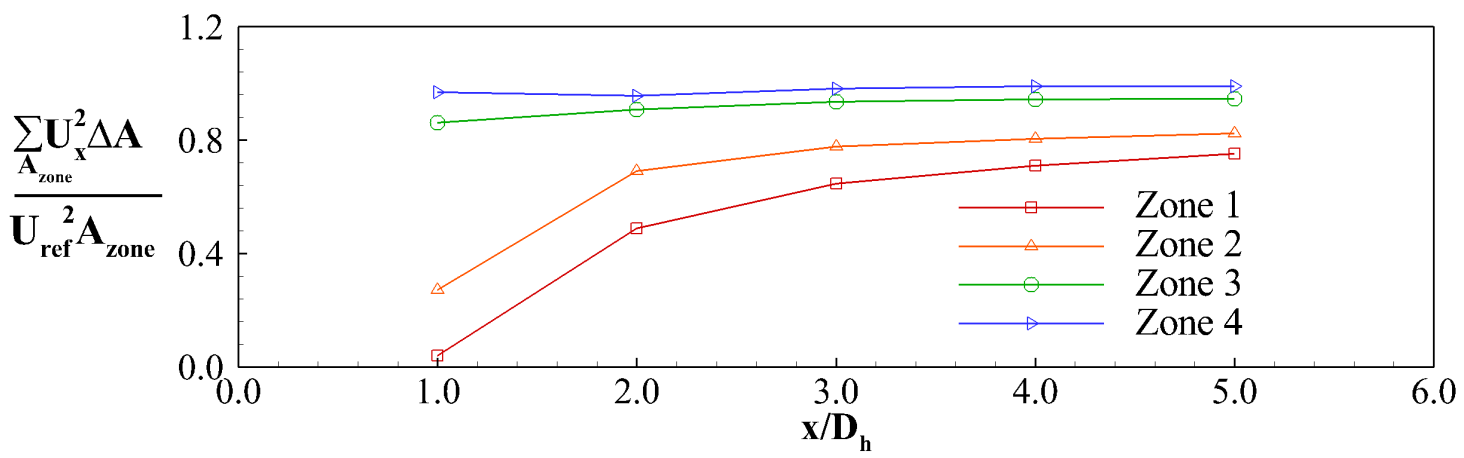

Figure 6.17: Axial momentum flux distribution in the wake for the straight-tail configuration in the absence of small-scale turbulence 
mixing of the wake is observed by comparing the trends in Figure 6.18 with those of the laminar case in Figure 6.17. Interestingly, compared to the baseline configuration, in the presence of a tail, the streamwise rate of mixing of the wake is less affected by smaller-scale turbulence.

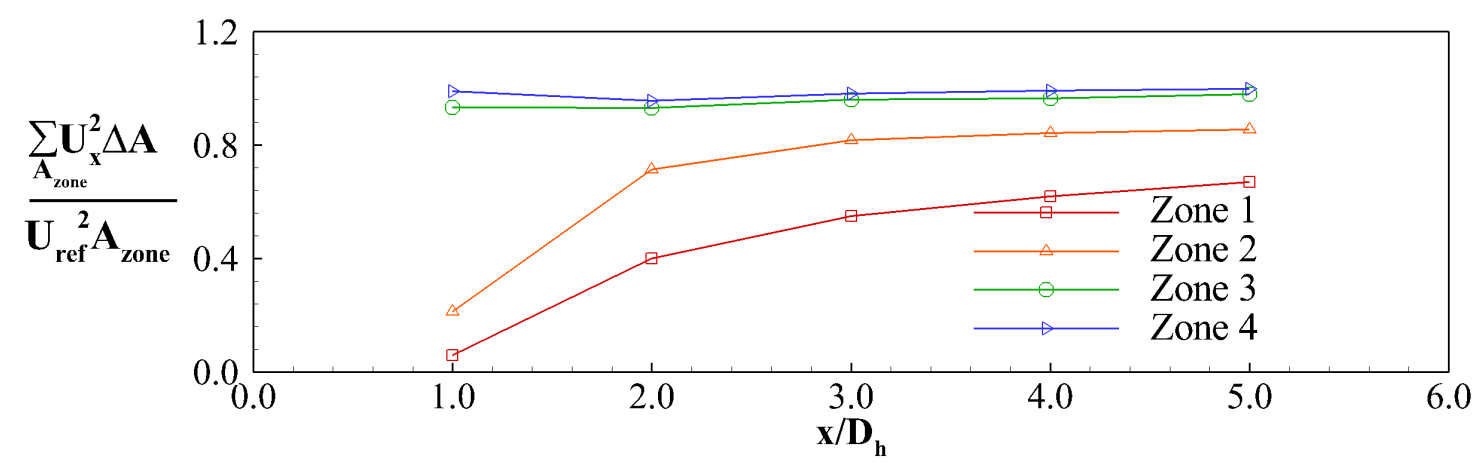

Figure 6.18: Axial momentum flux distribution in the wake for the straight-tail configuration in the presence of small-scale turbulence

\subsubsection{Lobed-Tail Configuration}

Shaping the trailing edge of the tail into lobes modifies the wake dynamics significantly as illustrated through isosurfaces of $\mathrm{Q}$ in Figure 6.19. As was noted in

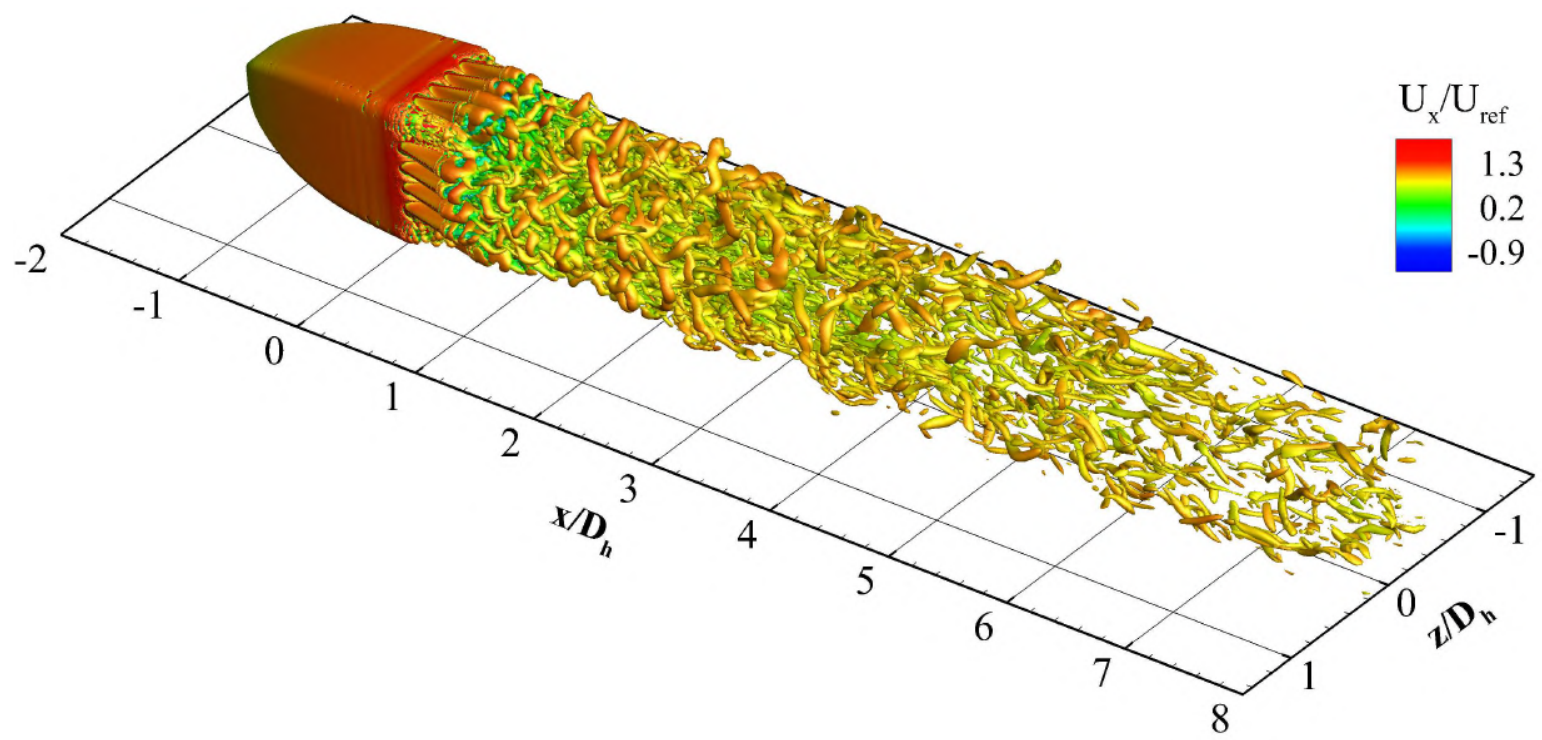

Figure 6.19: $\mathrm{Q} /\left(\mathrm{U}_{\text {ref }} / \mathrm{D}_{\mathrm{h}}\right)^{2}=9.5$ isosurfaces for the lobed-tail configuration in the absence of small-scale turbulence 
Chapter 3, lobed mixers promote mixing by increasing the contact surface area between the streams to be mixed and by developing additional coherent vortical structures. The increased surface area due to the lobes is observed to increase the cross sectional area of the wake significantly compared to that of the straight-tail configuration (Figure 6.12), to the extent that the cross section of the wake is now comparable to that of the no-tail baseline configuration (Figure 6.2). Accordingly, in the context of the manner a straight tail increases the base pressure, the presence of the lobes with the specific lobe geometries considered may offset the drag benefits of a tailed configuration.

The development of the so-called normal vortices, their streamwise tilting, and their deformation including the pinch-off effect of streamwise vortices shedding of the side walls of the lobes, all of which are well-established dynamics as reviewed in Chapter 3, produces a well-defined spanwise-periodic vorticity pattern immediately downstream of the lobes as illustrated in Figure 6.20. The contribution of these vortical structures to the wake dynamics becomes notably more evident when one compares the wake of the lobed-tail and straight-tail configurations for turbulent conditions (Figures 6.21 and 6.14). The streamwise periodic coherent vortical structures that occur at a Strouhal number of 0.7 for the straight-tail configuration

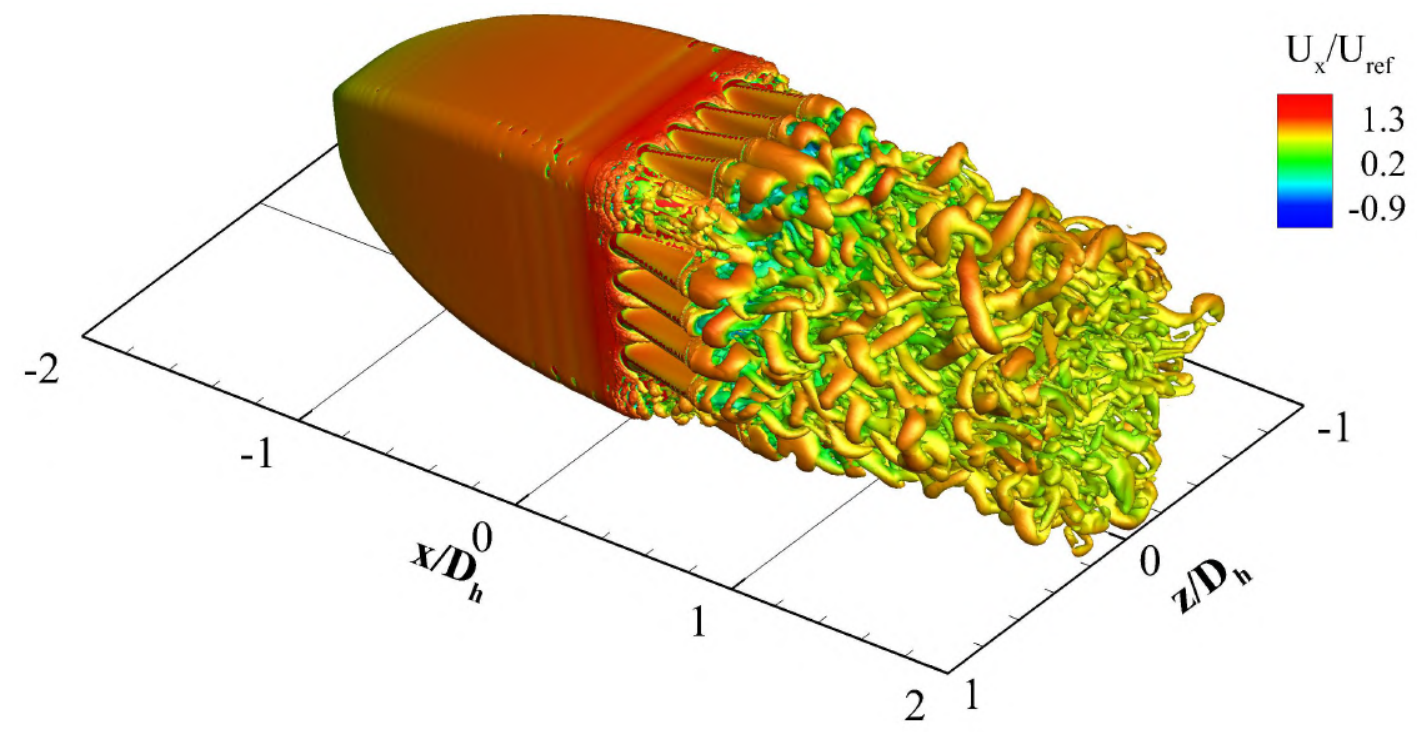

Figure 6.20: $\mathrm{Q} /\left(\mathrm{U}_{\text {ref }} / \mathrm{D}_{\mathrm{h}}\right)^{2}=9.5$ isosurfaces in the near wake for the lobedtail configuration in the absence of small-scale turbulence 


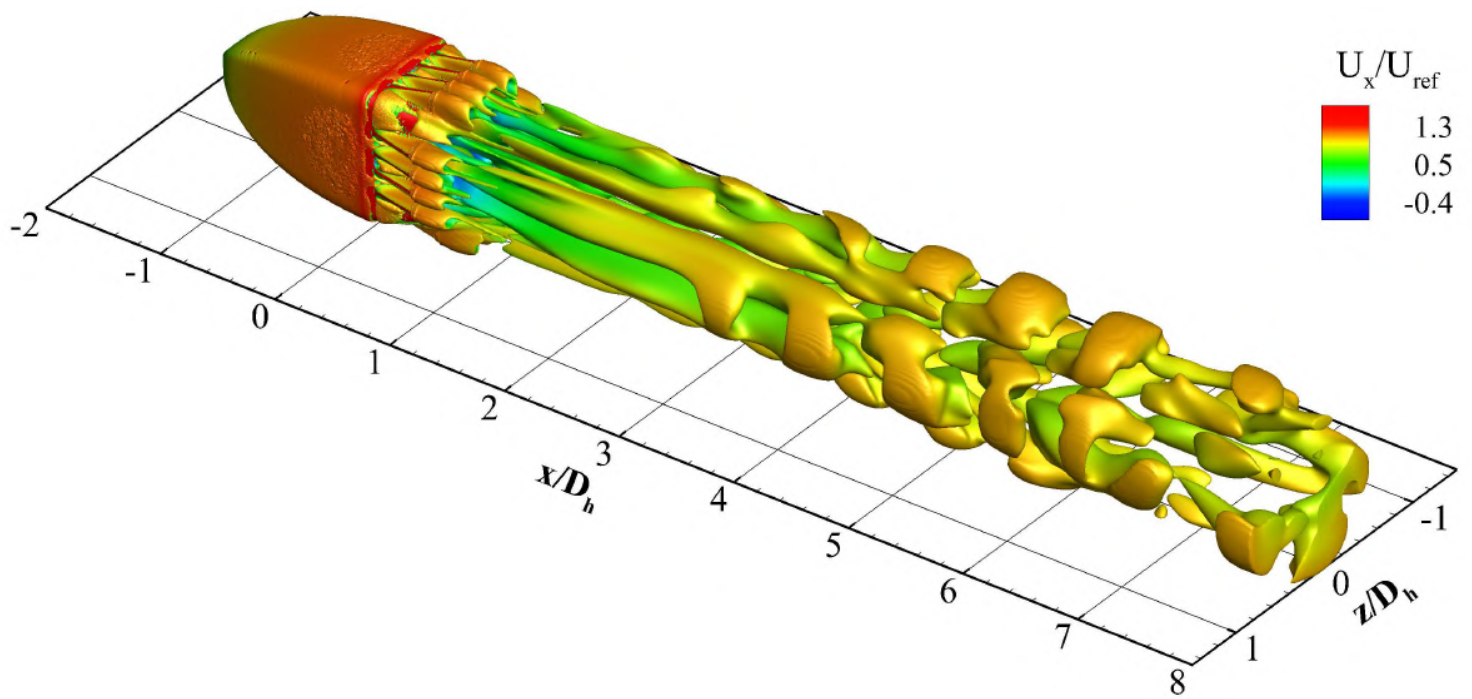

Figure 6.21: $\mathrm{Q} /\left(\mathrm{U}_{\mathrm{ref}} / \mathrm{D}_{\mathrm{h}}\right)^{2}=2.4 \times 10^{-2}$ isosurfaces for the lobed-tail configuration in the presence of small-scale turbulence

are observed to be replaced by streamwise periodic vortex loops that occur more frequently corresponding to a Strouhal number of 1.0.

On the basis of the lobes promoting cross-stream mixing, one would expect the distribution of axial momentum in the wake for the lobed tail to approach uniform conditions with streamwise distance more rapidly than for the straight tail. The trends for the lobed tail in Figure 6.22 do not compare with those for the straight tail in Figure 6.17 in a manner that is consistent with this expectation. This apparent discrepancy may be partially explained by the fact that while the lobes promote

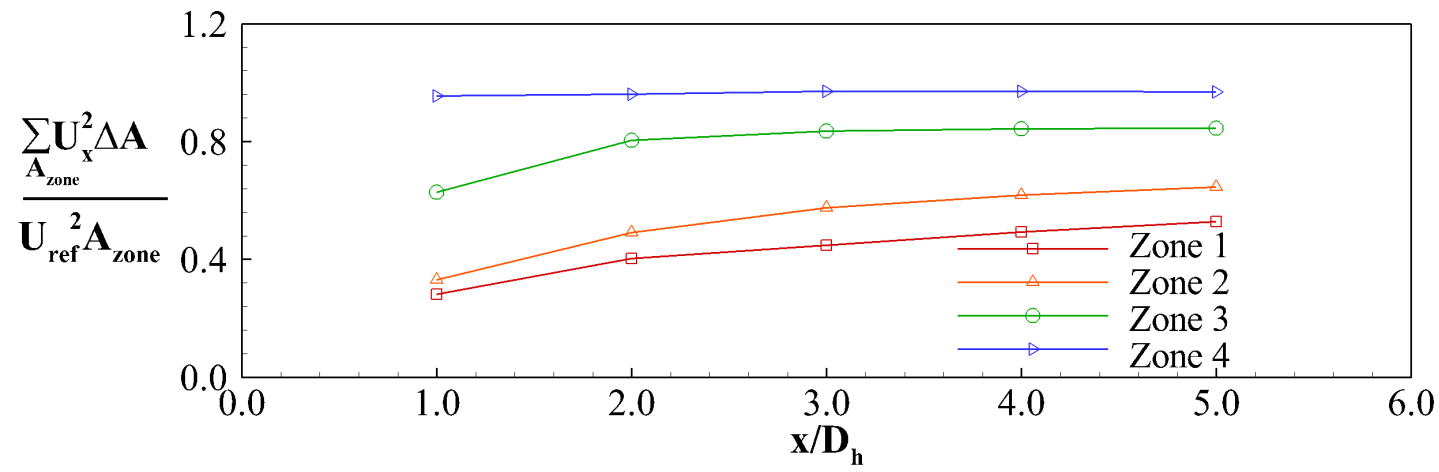

Figure 6.22: Axial momentum flux distribution in the wake for the lobedtail configuration in the absence of small-scale turbulence 
cross-stream mixing, they also produce an initially larger wake cross-section. The streamwise variation of the kinetic energy of transient structures for the lobed case, presented in Figure 6.23 suggests lower energy levels for the inner three zones compared to those with the straight tail (Figure 6.15), and this energy is observed to decrease notably more rapidly with the lobed tail than with the straight tail.

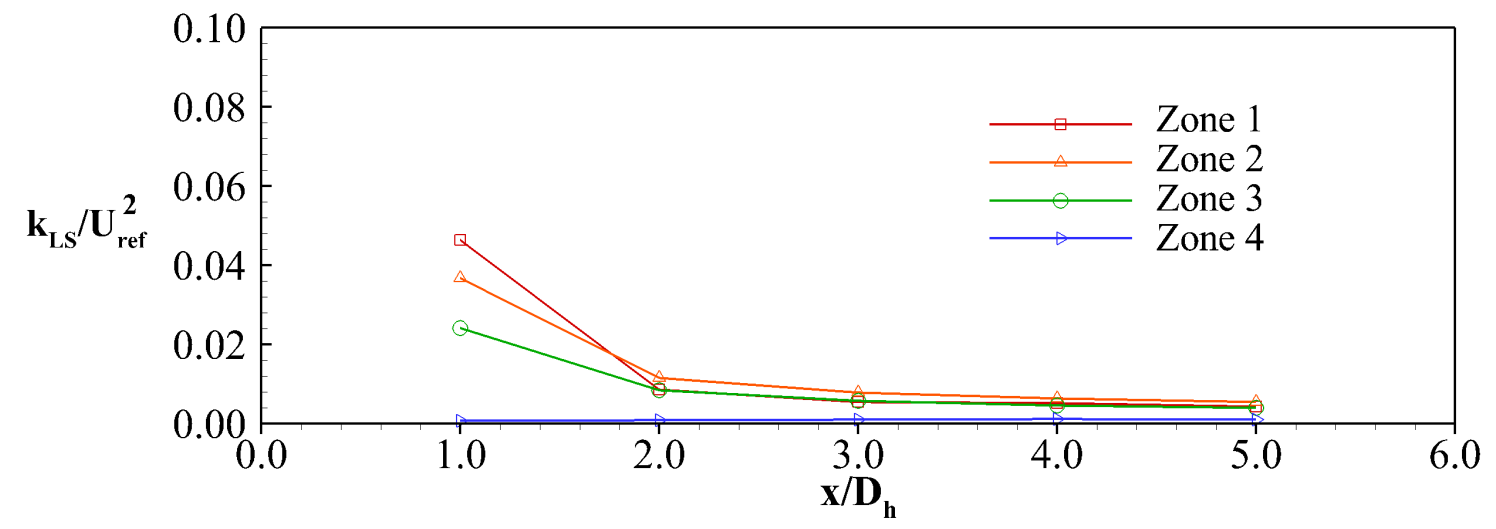

Figure 6.23: Kinetic energy of larger-scale transient structures in the absence of small-scale turbulence for the lobed-tail configuration

The length scale of the larger transient structures appears to be comparable for the lobed and straight tails as noted by comparing the trends in Figure 6.24 with those in Figure 6.16. On the basis of the noted kinetic energy and length scale trends, the extent of mixing promoted by the transient flow structures may be expected to be reduced for the lobed tail than for the straight tail, supporting the axial momentum

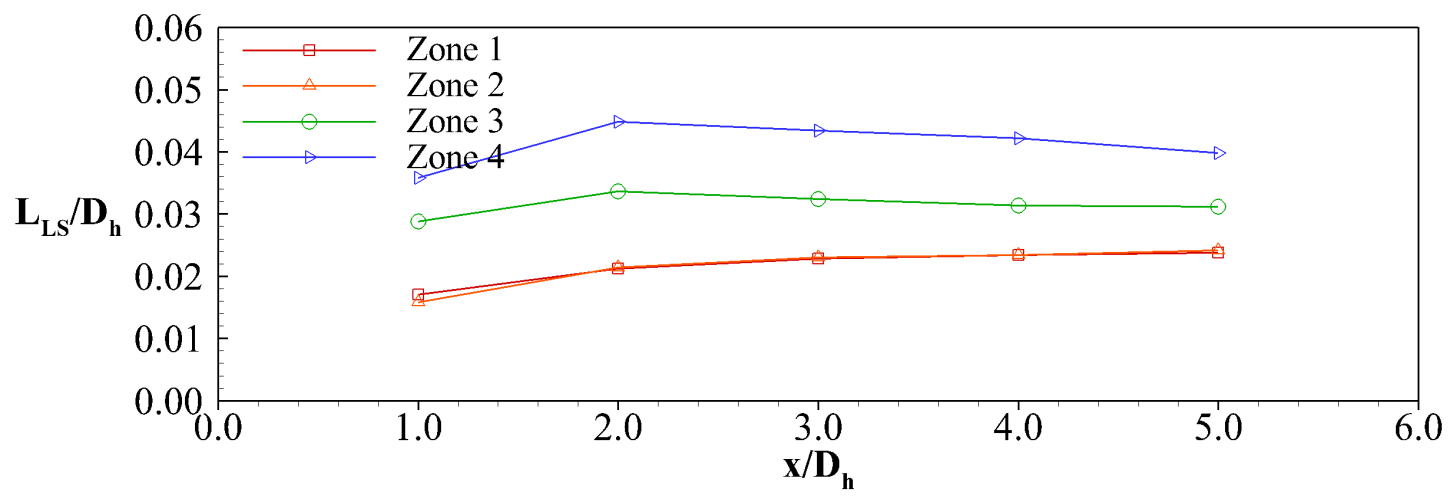

Figure 6.24: Length scale of larger-scale transient structures for the lobedtail configuration in the absence of small-scale turbulence 
trends noted in Figure 6.22 compared to those for the straight tail in Figure 6.17. The lower kinetic energy levels of the transient flow structures (Figure 6.23) despite their increased coherence (Figures 6.19 and 6.20) in the lobed-tail case compared to the straight-tail case warrants further investigation.

Based on the wake dynamics discussed in the foregoing, one would expect the body drag force for the straight-tailed configuration to be lower than for the baseline configuration, whereas the lobed-tail configuration to yield a drag force comparable to that of the baseline configuration. Calculation of the drag coefficients on the body for the three configurations confirmed these expected trends as summarized in Table 6.2. Furthermore, the predicted drag values are noted to compare favourably to the experimental values tabulated in Table 6.1 for the corresponding geometries of baseline, long-straight tail and long-large-lobed tail. This favourable comparison supports confidence in the computed results.

Table 6.2: Comparison of $C_{D}$ obtained from the simulations

\begin{tabular}{lccc}
\hline Configuration & Baseline & Straight-Tail & Lobed-Tail \\
\hline Laminar & 0.23 & 0.14 & 0.26 \\
Turbulent & 0.22 & 0.09 & 0.25 \\
\hline
\end{tabular}




\section{Chapter 7}

\section{Conclusions and Recommendations for Future Work}

The present study investigated the wake dynamics of a bluff body with a streamlined fore body and a rectangular blunt base of 1.15 aspect ratio. In the absence of small-scale turbulence, the near-wake development is shown to resemble that of a sphere rather than that of box-shaped bluff bodies of comparable base aspect ratios.

Specifically, the wake is observed to develop a helical pattern approximately aligned with the streamwise direction, which is the result of a local rotating instability within the recirculation region by the base of the body. Another dominant feature of the wake is the formation of vorticity rings immediately downstream of the edge of the base, prompted by the Kelvin-Helmholtz instability of the free shear layer that forms as an extension of the boundary layers of the body. The wavelength of the helical pattern and the formation frequency of the vortex rings are found to agree well with those prevailing in the wake of spheres. The noted wake structure is shown to persist at flow Reynolds numbers as high as 512,000 provided the small-scale turbulence in the flow is suppressed. In the presence of such turbulence, the helical pattern as well as periodic shedding of vorticity rings are found to be suppressed. In the laminar scenario, the kinetic energy of the larger-scale transient structures in the near wake are noted to be about $50 \%$ higher in the core of the wake than its peripheral regions. The length scale of the larger transient structures within five hydraulic diameters of the base, $\mathrm{L}_{\mathrm{LS}}$, is found to be of the order of $0.05 \mathrm{D}_{\mathrm{h}}$.

Investigation of the effects of plain and lobed tails on the wake structure formed the second part of the study. In the presence of a straight tail, the helical wake pattern observed for the no-tail configuration is weakened significantly. Boundary layer separation on the tail surfaces is observed to produce periodic streamwise grouping of spanwise vorticity through an inviscid instability, which is followed by formation of hairpin-like vortical structures through a secondary instability. The initial wake cross 
section is notably reduced in the presence of the tail, as the tail promotes movement of freestream fluid into the wake region. As with the baseline configuration, smallscale turbulence is observed to significantly suppress the dynamics driven by multiple instability modes in the laminar scenario. In the presence of small-scale turbulence, the wake is dominated by a steady recirculation zone in close vicinity of the base, and by streamwise periodic vortex loops farther downstream.

In the presence of lobes on the tail, the wake dynamics are observed to differ significantly from those of the straight-tail configuration. Specifically, the initial wake cross section is notably larger, with a size that is comparable to that of the no-tail configuration. The well-known coherent vortical structures associated with lobed mixers are observed to dominate the wake in close proximity of the trailing edge of the tail. There is no evidence of a helical pattern in the wake, which suggests that the rotational instability responsible for this pattern may no longer develop in the presence of the vortical structures created by the lobed trailing edge. The mixing enhancement effect of these vortical structures persists for a notable streamwise distance, including in the presence of small-scale turbulence. Despite this enhanced mixing, the dragreduction benefits of a straight tail are lost when lobes are added to the tail. This is likely caused by the lobes diverting the freestream away from the core of the wake while they develop vortical structures that promote cross-steam mixing.

In summary, the following are deemed to be the primary contributions of this study to the state-of-the-art of bluff-body wake dynamics:

- The instability modes well-known to prevail in the near wake of bluff bodies at low Reynolds numbers are shown to remain effective even at high Reynolds numbers if small-scale turbulence is suppressed.

- The streamwise-aligned helical shape that is well-known to occur in the wake of axisymmetric bodies due to an absolute instability is shown to be not restricted to axiymmetric bodies.

- A straight tail is shown to not only provide drag reduction benefits, but also weaken the larger-scale transient motions in the wake. 
- Adding a lobed pattern to the tail is shown to promote cross-stream mixing in the wake, with the vortical structures produced by the lobes dominating the wake. While the lobes are noted to offset the drag-reduction effect of the tail, they also offer an effective means of altering the hazard footprint of the wake.

The present investigation relied on the solution of the Navier-Stokes equations to shed light on the details of the wake dynamics. While the computational result for the laminar flow state are deemed very reliable, the results for the turbulent regime are affected by the prediction accuracy of the two-equation turbulence model utilized in the computations. Given the significant dissipative effect of small scale turbulence on the instability-driven wake dynamics observed in the computational results, it is deemed prudent that these observations be validated with detailed measurements of the bluff-body wake under laminar and turbulent conditions.

The aspect ratio of the bluff body in the present study was chosen to match the aspect ratio of the base of a standard tractor-trailer to allow the present study inform ongoing work on the aerodynamics of tractor trailers. In this context, applying the boat-tails of the present study to a wind tunnel model of a standard tractor trailer to establish the sensitivity of the wake dynamics to the initial conditions of the wake as defined by the flow field immediately upstream of the base of the body may be an informative next step.

The present study was conducted in the absence of a flat surface in proximity of the wake. It is well-known that ground proximity may have a significant effect on the wake dynamics of road vehicles. The present study may be readily extended to establish the effect of a modelled ground surface on the instability modes driving the rotational motion of the wake and the periodic shedding of vorticity rings off the base. 


\section{References}

[1] Patten, J., McAuliffe, B. R., Mayda, W., \& Tanguay, B. (2012). "Review of Aerodynamic Drag Reduction Devices for Heavy Trucks and Buses," NRC Laboratory Technical Report.

[2] McAuliffe, B. R. (2015). "Improving the aerodynamic efficiency of heavy duty vehicles: wind-tunnel test results of trailer-based drag-reduction technologies," NRC Laboratory Technical Report.

[3] Schaut, N. \& Sengupta, R. (2015). "Aerodynamic optimization of trailer add-on devices fully- and partially-skirted trailer configurations," SAE Inter. J. Commer. Vehicles, 8, pp. 695-704.

[4] California Air Resources Board, (2012). Final Regulation Order - Regulation to Reduce Greenhouse Gas Emmissions for Heavy-Duty Vehicles, Accessed: 2017-0714, URL: www.arb.ca.gov.

[5] Transport Canada, (2013). Regulations Amending the Motor Vehicle Safety Regulations (Standards 121, 206, 223 and 301.2), Canada Gazette Part II, 147(26), pp. 2899-2906.

[6] Transport Canada ecoTECHNOLOGY for Vehicles Program, Accessed: 2018-0820, URL: https://www.tc.gc.ca/en/programs-policies/programs/ecotechnologyvehicles-program.html.

[7] Taneda, S. (1956). "Experimental investigation of the wake behind cylinder and plates at low Reynolds numbers," J. Phys. Society of Japan, 11(3), pp. 302-307.

[8] Mair, W. A., \& Maull, D. J. (1971). "Bluff bodies and vortex shedding - a report on Euromech 17," J. Fluid Mech., 45, pp. 209-224.

[9] Bearman, P. W., \& Graham, J. M. R. (1980). "Vortex shedding from bluff bodies in oscillatory flow : A report on Euromech 119," J. Fluid Mech., 99, pp. 225-245.

[10] Berger, E., \& Wille, R. (1972). "Periodic flow phenomena," Ann. Rev. Fluid. Mech., 4, pp. 313-340.

[11] Ouro, P., Wilson, C. A., Evans, P., \& Angeloudis, A. (2017). "Large-eddy simulation of shallow turbulent wakes behind a conical island," Physics of Fluids, 29(12), pp. 1-15.

[12] Catalano, P., Wang, M., Iaccarino, G., \& Moin, P. (2003). "Numerical simulation of the flow around a circular cylinder at high Reynolds numbers," Intern. Journal of Heat and Fluid Flow, 24(4), pp. 463-469. 
[13] Bearman, P. W. (1984). "Vortex shedding from oscillating bluff bodies," Ann. Rev. Fluid Mech., 16(1), pp. 195-222.

[14] Parkinson, G. V. (1974). Mathematical models of flow-induced vibrations: Flow induced structural vibrations, p. 774, Berlin: Springer.

[15] Sarpkaya, T. (1979). "Vortex-induced ocillations," J. Appl. Mech., 46, pp. 241258.

[16] Gerrard, J. H. (1966). "The mechanics of the formation region of vortices behind bluff bodies," J. Fluid Mech., 25(2), pp. 401-413.

[17] Tanner, T. (1972). "A method for reducing the base drag of wings with blunt trailing edges," Aeronaut. Q., 23, pp. 15-23.

[18] Roshko, A. (1954). "On the drag and shedding frequency of two-dimensional bluff bodies" NACA TN, $\mathbf{3 1 6 9}$.

[19] Roshko, A. (1955). "On the wake and drag of bluff bodies," J. Aeronautical Sci., 22(2), pp. 124-132.

[20] Bearman, P. W., \& Owen, J. C. (1997). "Reduction of bluff-body drag and suppression of vortex shedding by the introduction of wavy separation lines," J. Fluids and Structures, 12, pp. 123-130.

[21] Darekar, R. M., \& Sherwin, S. J. (2000). "Flow past a square-section cylinder with a wavy stagnation face," J. Fluid Mech., 426, pp. 263-295.

[22] Gilliron, P., \& Kourta, A. (2010). "Aerodynamic drag reduction by vertical splitter plates," Exp. Fluids, 48(1), pp. 1-16.

[23] Beaudoin, J. F., \& Aider, J. L. (2008). "Drag and lift reduction of a 3D bluff body using flaps," Exp. Fluids, 44(4), pp. 491-501.

[24] Huerre, P. (2000). "Open shear flow instabilities," Perspectives in Fluid Dynamics, pp. 159-229.

[25] Huerre, P., \& Monkewitz, P. A. (1990). "Local and global instabilities in spatially developing flows," Annu. Rev. Fluid Mech., 22, pp. 473-537.

[26] Pier, B. (2002). "On the frequency selection of finite-amplitude vortex shedding in the cylinder wake," J. Fluid Mech., 458, pp. 407-417.

[27] Pier, B. (2008). "Local and global instabilities in the wake of a sphere," J. Fluid Mech., pp. 39-61, Cambridge: University Press.

[28] Thompson, M. C. (2006). "Wake transition of two-dimensional cylinders and axisymmetric bluff bodies," J. Fluids and Structures, 22, pp. 793-806. 
[29] Roos, F. W., \& Willmarth, W. W. (1971). "Some experimental results on sphere and disk drag," AIAA Journal, 9(2), pp .285-291.

[30] Natarajan, R., \& Acrivos, A. (1993). "The instability of the steady flow past spheres and disks," J. Fluid Mech., 254, pp. 323-344, Cambridge: University Press.

[31] Taneda, S. (1978). "Visual observations of the flow past a sphere at Reynolds numbers between $10^{4}$ and $10^{6}, "$ J. Fluid Mech., 85, pp. 187-192.

[32] Mittal, R. (1998). "Planar symmetry in the unsteady wake of a sphere," AIAA Journal, 37(3), pp. 387-390.

[33] Magarvey, R. H., \& Bishop, R. L. (1961). "Wakes in liquid-liquid systems," Phys. Fluids, 4, pp. 800-805.

[34] Nakamura, I. (1976). "Steady wake behind a sphere," Phys. Fluids, 19, pp. 1-8.

[35] Sakamoto, H., \& Haniu, H. (1995). "The formation mechanism and shedding frequency of vortices from a sphere in uniform shear flow," J. Fluid Mech., 287, pp. 151-171.

[36] Ormires, D. \&, Provansal, M. (1999). "Transition to turbulence in the wake of a sphere," Phys. Revi. Letters.

[37] Möller, W. (1938). "Experimentelle untersuchung zur hydromechanik der Kugel."

[38] Achenbach, E. (1974). "Vortex shedding from spheres," J. Fluid Mech., 62, pp. 209-221.

[39] Monkewitz, P. A. (1987). "A note on vortex shedding from axisymmetric bluffbodies," J. Fluid Mech., 192, pp. 561-575.

[40] Kim, K. J., \& Durbin, P. A. (1988). "Observation of the frequencies in a sphere wake and drag increase by acoustic excitation," Phys. Fluids, 31(11), pp. 32603265 .

[41] Ghidersa, B., \& Dusek, J. (2000). "Breaking of axisymmetry and onset of unsteadiness in the wake of s sphere," J. Fluid Mech., 423, pp. 33-69.

[42] Sakamoto, H., \& Haniu, H. (1990). "A study on vortex shedding from spheres in a uniform flow," J. Fluids Eng., 112(4), pp. 386-392.

[43] Berger, E., Scholz, D., \& Schumm, M. (1989). "Coherent vortex structures in the wake of a sphere and a circular disk at rest and under forced vibrations," J. Fluids and Structures, 4(3), pp. 231-257. 
[44] Tomboulides A. G., \& Orszag, S. A. (2000). "Numerical investigation of transitional and weak turbulent flow past a sphere," J. Fluid Mech., 416, pp. 45-73.

[45] Sevilla, A., \& Martinez-Bazan, C. (2004). "Vortex shedding in high Reynolds number axisymmetric bluff-body wakes: local linear instability and global bleed control," Phys. Fluids, 16(9), pp. 3460-3469.

[46] Pier, B. (2013). "Periodic and quasiperiodic vortex shedding in the wake of a rotating sphere," J. Fluids and Structures, 41, pp. 43-50.

[47] Skarysz, M., Rokicki, J., Goujon-Durand, S., \& Wesfreid, J.E. (2018). "Experimental investigation of the wake behind a rotating sphere," Phys. Revi. Fluids.

[48] Fabre, D., August, F., \& Magnaudet, J. (2008). "Bifurcations and symmetry breaking in the wake of axisymmetric bodies," Phys. Fluids, 20(5).

[49] Meliga, P., Chomaz, J.M., \& Sipp, D. (2009). "Global mode interaction and pattern selection in the wake of a disk: a weakly nonlinear expansion," J. Fluid Mech., 633, pp. 159-189.

[50] Marshall, D., \& Stanton, T. E. (1931). "On the eddy system in the wake of flat circular plates in three dimensional flow," Proc. R. Soc. Lond., 130, pp. 295-301.

[51] Bury, Y., \& Jardin, T. (2012). "Transitions to chaos in the wake of an axisymmetric bluff body," Phys. Letters A, pp. 3219-3222.

[52] Rigas, G., Oxlade, A. R., Morgans, A. S., \& Morrison, J. F. (2014). "Lowdimensional dynamics of a turbulent axisymmetric wake," J. Fluid Mech., 755.

[53] Rigas, G., Brackston, R. D., Morgans, A. S., \& Morrison, J. F. (2015). "Difusive dynamics and stochastic models of turbulent axisymmetric wakes," J. Fluid Mech., 778 .

[54] Roshko, A. (1954). "On the development of turbulent wakes from vortex streets," NASA Technical Reports.

[55] Provansal, M., Mathis, C., \& Boyer, L. (1987). "Benard-von Karman instability: transient and forced regimes," J. Fluid Mech., 182, pp. 1-22.

[56] Gerrard, J. H. (1978). "The wakes of cylindrical bluff bodies at low Reynolds number," Trans. Royal Society of London, 288(1354), pp. 351-382.

[57] Triantafyllou, G. S., Triantafyllou, M. S., \& Chryssostomidis, C. (1986). "On the formation of vortex streets behind stationary cylinders," J. Fluid Mech., 170, pp. 461-477.

[58] Monkewitz, P. A., \& Nguyen, L. N. (1987). "Absolute instability in the near-wake of two-dimensional bluff bodies," J. Fluids and Structures, 1(2), pp. 165-184. 
[59] Chomaz, J. M., Huerre, P., \& Redekopp, L. G. (1988). "Bifurcations to local and global modes in spatially developing flows," Phys. Rev. Letters, 60(1), p. 25.

[60] Plaschko, P., Berger, E., \& PeraltaFabi, R. (1993). "Periodic flow in the near wake of straight circular cylinders," Phys.Fluids A: Fluid Dynamics, 5(7), pp. 1718-1724.

[61] Koch, W. (1985). "Local instability characteristics and frequency determination of self-excited wake flows," J. Sound and Vibration, 99(1), pp. 53-83.

[62] Williamson, C. H. K. (1988). "Defining a universal and continuous StrouhalReynolds number relationship for laminar vortex shedding of a circular cylinder," Phys. Fluids, 31, pp. 2742-2744.

[63] König, M., Eckelmann, H. (1992). "Visualisation of the spanwise cellular structure of the laminar wake of wall-bounded circular cylinders," Phys. Fluids, 4, pp. 869-872.

[64] Hammache, M., \& Gharib, M. (1991). "An experimental study of the parallel and oblique vortex shedding from circular cylinders," J. Fluid Mech., 232, pp. 567-590.

[65] Thompson, M, Hourigan, \& K., Sheridan, J. (1994). "Three-dimensional instabilities in the cylinder wake," Int. Colloq. Jets, Wakes, Shear Layers.

[66] Zhang, H., Fey, U., Noack, B. R., Konig, M., \& Eckelmann, H. (1995). "On transition of the cylinder wake," Phys. Fluids, 7(4), pp. 779-794.

[67] Williamson, C. H. K. (1988). "The existence of two stages in the transition to three-dimensionality of a cylinder wake," Phys. Fluids, 31, pp. 3165-3168.

[68] Williamson, C. H. K. (1992). "The natural and forced formation of spot-like vortex dislocations in the transition of a wake," J. Fluid Mech., 182, pp. 393-441.

[69] Robichaux, J., Balachandar, S., \& Vanka, S. P. (1999). "Three-dimensional Floquet instability of the wake of a square cylinder," Phys. Fluids, 11, pp. 560-578.

[70] Hourigan, K., Thimpson, M. C., \& Tan, B. T. (2001). "Self-sustained oscillations in flows around long blunt plates," J. Fluids and Structures, 15.

[71] Barkley, H., \& Henderson, R. D. (1996). "Three-dimensional Floquet stability analysis of the wake of a circular cylinder," J. Fluid Mech., 322, pp. 215-241.

[72] Williamson, C. H. K. (1995). "Scaling of streamwise vortices in wakes," Phys. Fluids, 7(10), pp. 2307-2309. 
[73] Thompson, M. C., Leweke, T., \& Williamson, C. H. K. (2001). "The physical mechanism of transition in bluff body wakes," J. Fluids and Structures, 15, pp. 607-616.

[74] Bloor, M. S. (1964). "The transition to turbulence in the wake of a circular cylinder," Journal of Fluid Mech., 19(2), pp. 290-304.

[75] Williamson, C. H. K. (1996). "Vortex dynamics in the cylinder wake," Ann. Rev. Fluid Mech., 28, pp. 477-539.

[76] Bearman, P. W. (1969). "On vortex shedding from a circular cylinder in the critical Reynolds number rgime," J. Fluid Mech., 37(3), pp. 577-585.

[77] Schewe, G. (1983). "On the force fluctuations acting on a circular cylinder in crossflow from subcritical up to transcritical Reynolds numbers," J. Fluid Mech., 133, pp. 265-285.

[78] Roshko, A. (1993). "Perspectives on bluff body aerodynamics," J. Wind Eng. and Indust. Aerodynamics, 49(1-3), pp. 49-79.

[79] Roshko, A. (1961). "Experiments on the flow past a circular cylinder at very high Reynolds number," J. Fluid Mech., 10(3), pp. 345-356.

[80] Ahmed, S.R., Ramm, G., \& Faltin, G. (1984). "Some salient features of the time-averaged ground vehicle wake," SAE Transactions, 93(2).

[81] Grandemange, M., Cadot, O., \& Gohlke, M. (2012). "Reflectional symmetry breaking of the spectral flow over three-dimensional bluff bodies," Phys. Rev. E, 86.

[82] Grandemange, M., Gohlke, M., \& Cadot, O. (2013). "Bi-stability in the turbulent wake past parallelepiped bodies with various aspect ratios and wall effects," Phys. Fluids, 25.

[83] Herry, B., Keirsbluck, L., Labraga, L, \& Paquet, J. B. (2011). "Flow bistability downstream of three-dimensional double backward facing steps at zero-degree sideslip," Trans. ASME J. Fluids Eng., 133(5).

[84] Cadot, O., Evrard, A., \& Pastur, L. (2015). "Imperfect supercritical bifurcation in a three-dimensional turbulent wake," Phys. Rev. E, 91.

[85] Johnson, T.A., \& Patel, V. C. (1999). "Flow past a sphere up to a Reynolds number of 300," J. Fluid Mech.," 378, pp. 19-70.

[86] Fabre, D., Auguste, F., \& Magnaudet, J. (2008). "Bifurcations and symmetry breaking in the wake of axisymmetric bodies," Phys. Fluids, 20. 
[87] Grandemange, M., Gohlke, M., \& Cadot, O. (2014). "Statistical axisymmetry of the turbulent sphere wake," Exp. Fluids, 55.

[88] Barkley, D., Tuckerman, L. S., \& Golubitsky, M. (2000). "Bifurcation theory for three-dimensional flow in the wake of a circular cylinder," Phys. Rev. E, 61(5), p. 5247.

[89] Brackston, R. D., Garcia de la Cruz, J. M., Wynn, A., Rigas, G., \& Morrison, J. F. (2016). "Stochastic modelling and feedback control of bistability in a turbulent bluff body wake," J. Fluid Mech., 802, pp. 726-749.

[90] Evrard, A., Cadot, O., Herbert, V., Ricot, D., Vigneron, R., \& Delery, J. (2016). "Fluid force and symmetry breaking modes of a 3D bluff body with a base cavity," J. Fluids and Structures, 61, pp. 99-114.

[91] Brackston, R. D., Wynn, A., \& Morrison, J. F. (2018). "Modelling and feedback control of vortex shedding for drag reduction of a turbulent bluff body wake," Inter. J. Heat and Fluid Flow, 71, pp. 127-136.

[92] Volpe, R., Devinant, P., \& Kourta, A. (2015). "Experimental characterization of the unsteady natural wake of the full-scale square back Ahmed body: Flow Bi-Stability and Spectral Analysis," Exp. Fluids, pp. 56-99.

[93] Grandemange, M., Gohlke, M., \& Cadot, O. (2013). "Turbulent wake past a three-dimensional blunt body. part 1. global modes and bi-stability," J. Fluid Mech., 722, pp. 51-84.

[94] Marquet, O., \& Larsson, M. (2015). "Global wake instabilities of low aspect-ratio flat-plates," European J. Mechanics-B/Fluids, 49, pp. 400-412.

[95] Saltzman, E.J., \& Meyer, R. R. Jr. (1974). "Drag reduction obtained by rounding vertical corners on a box-shaped ground vehicle," NASA Technical Report.

[96] Martini, H., Bergqvist, B., Hjelm, L., \& Lfdahl, L. (2011). "Aerodynamic effects of roof deflector and cab side extenders for truck-trailer combinations," SAE Technical Paper.

[97] Leuschen, J. (2013). "Considerations for the wind tunnel simulation of tractortrailer combinations: correlation of full- and half-scalemeasurements," SAE Int. J. Commer. Veh., 6, pp. 529-538.

[98] Landman, D., Wood, R., Seay, W., \& Bledsoe, J. (2010). "Understanding practical limits to heavy truck drag reduction," SAE Int. J. Commer. Veh., 2, pp. 183-190.

[99] Wood, R. (2009). "Operationally-practical and aerodynamically-robust heavy truck trailer drag reduction technology," SAE Int. J. Commer. Veh., 1, pp. 237-247. 
[100] McAuliffe, B. R. (2016). "Potential for the accumulation of ice and snow for a boat-tail equipped heavy-duty vehicle," SAE Technical Paper.

[101] Martini, H., Bergqvist, B., Hjelm, L., \& Lfdahl, L. (2016). "Aerodynamic performance of flat-panel boat-tails and their interactive benefits with side-skirts," SAE Technical Paper.

[102] Martini, H., Bergqvist, B., Hjelm, L., \& Lfdahl, L. (2011). "Influence of different truck and trailer combinations on the aerodynamic drag," SAE Technical Paper.

[103] McAuliffe, B. R., \& Wall, A. S. (2016). "Aerodynamic performance of flat-panel boat-tails and their interactive benefits with side-skirts," SAE Inter. J. Commer. Vehicles, 9, pp. 70-82.

[104] Morel, T. (1979). "Effect of base cavities on the aerodynamic drag of an axisymmetric cylinder," Aeronautical Quarterly, 30(2), pp. 400-412.

[105] Viswanath, P. R. (1996). "Flow management techniques for base and afterbody drag reduction," Progress in Aerospace Sciences, 32(2-3), pp 79-129.

[106] Waitz, I. A., Qui, T. A., Manning, T. A., Fung, A. K. S., Elliot, J. K., Kervin, J. M., Krasnodebski, J. K., O’Sullivan, M. N., Tew, D. E., Greitzer, E. M., Marble, F. E., Tan, C. S., \& Tillman, T. G. (1997). "Enhanced mixing with streamwise vorticity," Prog. Aerospace Sci., 33, pp. 323-351.

[107] Zaman, K. B. M. Q., Wang, F. Y., \& Georgiadis, N. J. (2003). "Noise, turbulence and thrust of subsonic freejets from lobed nozzles," AIAA Journal, 41, pp. 398-407.

[108] Paterson, R. W. (1984). "Turbofan forced mixer nozzle flow field a benchmark experimental study," J. Engineering for Gas Turbines and Power, 106, pp. 692-698.

[109] Werle, M. J., Presz,W., \& Paterson, R. (1987). "Flow structure in an periodic axial vortex array, 25th AIAA Aerospace Sciences Meeting, AIAA Paper 1987-610.

[110] Eckerle, J. K., Sheibani, H., \& Awad, J. (1992). "Experimental measurement of the vortex development downstream of a lobed forced mixer," J. Eng. for Gas Turbines and Power, 114, pp. 63-71.

[111] Smith, L. Majamaki, A., Lan, I., Delabroy, O., Karagozian, A., Marble, F., \& Smith, O. (1997). "Mixing enhancement in a lobed injector" Phys. Fluids, 9, pp.667-678.

[112] Elliott, J. K., Manning, T. A., Qiu, Y. J., Greitzer, E. M., Tan, C. S., \& Tillman, T. G. (1992). "Computational and experimental studies of flow in multi-lobed mixers," 28th Joint Propulsion Conference and Exhibit, AIAA Paper 1992-3568. 
[113] Barber, T., Paterson, R. W., \& Skebe, S. A. (1988). "Turbofan forced mixer lobe flow modeling. Part 1. experimental and analytical assessment, NASA CR-4147.

[114] Skebe, S., Paterson, R., \& Barber, T. (1988). "Experimental investigation of three-dimensional forced mixer lobe flow fields, 1st National Fluid Dynamics Conference, AIAA Paper 1988-3785.

[115] O'Sullivan, M. N., Krasnodebski, J. K., Waitz, I. A., Greitzer, E. M., Tan, C. S., \& Dawes,W. N. (1996). "Computational study of viscous effects on lobed mixer flow features and performance," J. Propulsion and Power, 12, pp. 449-456.

[116] Brinkerhoff, J., \& Yaras, M. I. (2013). "An experimental and computational study of mixing mechanisms in sn sxisymmetric lobed mixer," J. Propulsion and Power, 29(5), pp. 1017-1030.

[117] Ooba, Y., Kodama, H., Nakamura, Y., Nozaki, O., Yamamoto, K., \& Nishizawa, T. (2002). "Large eddy simulation analysis of a 18-lobe convoluted mixer nozzle, 40th AIAA Aerospace Sciences Meeting and Exhibit, AIAA Paper 2002-0717.

[118] McVey, J. B., \& Kennedy, J. B. (1989). "Flame propagation enhancement through streamwise vorticity stirring," 27th Aerospace Sciences Meeting, AIAA Paper 1989-619.

[119] Waitz, I. A., \& Underwood, D. S. (1995). "Effect of heat release on streamwise vorticity enhanced mixing," J. Propulsion and Power, 12, pp. 638-645.

[120] Winant, C. D., \& Browand, F. K. (1974). "Vortex pairing: the mechanism of turbulent mixing-layer growth at moderate Reynolds number," J. Fluid Mech., 63, pp. 237-255.

[121] Ho, C. M., \& Huerre, P. (1984). "Perturbed free shear layers, Annu. Rev. of Fluid Mechanics, 16, pp. 365-424.

[122] McCormick, D. C., \& Bennett, J. C. (1994). "Vortical and turbulent structure of a lobed mixer free shear layer," AIAA Journal, 32, pp. 18521859.

[123] Glauser, M., Ukeiley, L., \& Wick, D. (1996). "Investigation of turbulent flows via pseudo flow visualization, part 2 the lobed mixer flow field, Exp. Thermal and Fluid Science, 13, pp. 167-177.

[124] Ukeiley, L., Varghese, M., Glauser, M., \& Valentine, D. (1992). "Multifractal analysis of a lobed mixer flow field utilizing the Proper Orthogonal Decomposition," AIAA Journal, 30, pp. 1260-1267.

[125] Hu, H., Saga, T., Kobayashi, T., \& Taniguchi, N. (2001). "A study on a lobed jet mixing flow by using stereoscopic Particle Image Velocimetry technique," Phys. Fluids, 13, pp. 34253441. 
[126] Rae, W. H., \& Pope, A. (1984). Low speed wind tunnel testing, John Wiley and Sonds, Second Edition.

[127] Katz, J. (1982). Automotive aerodynamics, First edition, San Diego State University, USA.

[128] Broughton, C. A. (1990). Experimental investigation of slotted wall wind tunnel test sections for low interference road vehicle testing, Master of Eng. Thesis, Carleton University.

[129] Schultz-Hausmann, F. K., \& Vagt, J. D. (1988). "Influence of test-section length and collector area on measurements in 3/4 open-jet automotive wind tunnels," SAE International Congress.

[130] Van Every, D., \& Harris, J. L. (1990). "Slotted-wall research with disk and parachute models in the DSMA low-speed wind tunnel," Sandia National Labs., Albuquerque, NM (USA); DSMA International, Inc., Mississauga, ON (Canada).

[131] Raimondo, S., \& Clark, P. J. F. (1982). "Slotted wall test section for automotive aerodynamic facilities," AIAA $12^{\text {th }}$ Aerodynamic Testing Conference.

[132] Clark, P. J. F., (1994). "Wall interference correction to drag measurements in automotive wind tunnels," J. Wind Eng. and Industrial Aerodynamics.

[133] Templin, J. T., \& Raimondo, S. (1986). "Experimental evaluation of test section boundary interference effects in road vehicle tests in wind tunnels," J. Wind Eng. and Industrial Aerodynamics, 22(2-3), pp. 129-148.

[134] Williams, C. D. (1975). A new slotted-wall method for producing low boundary corrections in two-dimensional airfoil testing, $\mathrm{PhD}$ Thesis, University of British Columbia.

[135] Flay, R. G. J, Elfstrom, G. M., \& Clark, P. J. F. (1983). "Slotted-wall test section for car and truck aerodynamic tests: interim report," Proc. ASME Conference on Aerodynamics of Transportation.

[136] Flay, R. G. J, Elfstrom, G. M., \& Clark, P. J. F. (1983). "Slotted-wall test section for automotive tests at yaw," SAE International Congress.

[137] Nelson, W. J., \& Cubbage, J. M. (1957). "Effects of slot size and geometry on the flow in rectangular tunnels at Mach numbers up to 1.4," NASA Research Memorandum, Langley Aeronautical Laboratory, USA.

[138] Berndt, S. B. (1977). "Inviscid Theory of wall interference in slotted test sections," The Aeronautical Research Institute of Sweden.

[139] Olander, M. (2011). CFD simulation of the Volvo cars slotted walls wind tunnel, Master's Thesis in Solid and Fluid Mechanics, Chalmers University,. 
[140] Brooks, C.W., Harris, C.D., \& Reagon, P.G. (1994). The NASA Langley 8-Foot Transonic Wind Pressure Tunnel Calibration.

[141] Biswas, R., \& Strawn, R. C. (1998). "Tetrahedral and hexahedral mesh adaptation for CFX problems," Applied Numerical Mathematics, 26(1-2), pp. 135-151.

[142] Katz, A., \& Sankaran, V. (2011). "Mesh quality effects on the accuracy of CFD solutions on unstructured meshes," J. Comp. Phys., 230(20), pp. 7670-7686.

[143] Menter, F. R., (1994). "Two-equation eddy-viscosity turbulence models for engineering applications," AIAA Journal, 32(8), pp. 1596-1605.

[144] Menter, F., \& Esch, T. (2001). "Elements of industrial heat transfer predictions," 16th Brazilian Congress of Mechanical Engineering (COBEM), 109, p. 650 .

[145] Rhie, C. M. \& Chow, W. L. (1983). "Numerical study of the turbulent flow past an airfoil with trailing edge separation," AIAA Journal, 21(11), pp. 1525-1532.

[146] Ferziger, J. H., \& Peric, M. (2002). Computational methods for fluid dynamics, Springer-Verlag Berlin Heidelberg.

[147] ANSYS, (2006). ANSYS CFX-Solver theory guide, 11 ${ }^{\text {th }}$ ed., ANSYS, Inc., 275 Technology Drive, Canonsburg, PA.

[148] Kehs, J. P., Visser, K. D., Grossman, J., Niemiec, J., Smith, A., \& Horrell, C. M. (2013). "A Comparison of full scale aft cavity drag reduction concepts with equivalent wind tunnel test results," SAE Inter. J. Commer. Vehicles, 6, pp. 486-497.

[149] Schlipf, D., Trabucchi, D., Bischoff, O., Hofs, M., Mann, J., Mikkelsen, T., \& Khn, M. (2010). "Testing of frozen turbulence hypothesis for wind turbine applications with a scanning lidar system," In 15th Inter. Symposium for the Advancement of Boundary Layer Remote Sensing, ISARS.

[150] McAuliffe, B. R., \& Yaras, M. I. (2008). "Numerical study of instability mechanisms leading to transition in separation bubbles," J. Turbomachinery, 130(2), 021006 .

[151] Warming, R. F., \& Beam, M. (1976). "Upwind second-order difference schemes and applications in aerodynamic flows," AIAA Journal, 14(9), pp. 12411249.

[152] Patankar, S. V. (1981). Numerical heat transfer and fluid flow, CRC press.

[153] Bogdanof, D. W. 1983. "Compressibility effects in turbulent shear layers," AIAA Journal, 21, pp. 926-927. 
[154] Papamoschou, D. \& Roshko, A. (1986). "Observations of supersonic free shear layers," AIAA Paper, 86-0162.

[155] Papamoschou, D. \& Roshko, A. (1988). "The compressible turbulent shear layer: an experimental study," J. Fluid Mechanics, 197, pp. 453-477.

[156] Elliot, G. S., \& Samimy, M. (1988). "Compressible effects in free shear layers," Phys. Fluids: Fluid Dynamics 2.

[157] Gutmark, E., Schadow, K. C., \& Wilson, K. J. (1991). "Effect of convective Mach number on mixing of coaxial circular and rectangular jets," Phys. Fluids A: Fluid Dynamics, 3(1), pp. 29-36.

[158] Zhuang, M., Kubota, T., \& Dimotakis, P. E. (1988). "On the instability of inviscid, compressible free shear layers," AIAA Journal, 28(10), pp. 1728-1733.

[159] Papamoschou, D. (1991). "Structure of the compressible turbulent shear layer," AIAA journal, 29(5), pp. 680-681.

[160] Ragab, S. A., \& Wu, J. L. (1989). "Linear instabilities in twodimensional compressible mixing layers. Phys. Fluids A: Fluid Dynamics, 1(6), pp. 957-966.

[161] Lessen, M., Fox, J. A., \& Zien, H. M. (1965). "On the inviscid stability of the laminar mixing of two parallel streams of a compressible fluid," J. Fluid Mech., 23(2), pp. 355-367.

[162] Lele, S. (1989). "Direct numerical simulation of compressible free shear flows," In 27th Aerospace Sci. Meeting, p. 374.

[163] Sandham, N. D., \& Reynolds, W. C. (1991). "Three-dimensional simulations of large eddies in the compressible mixing layer," J. Fluid Mech., 224, pp. 133-158.

[164] Brown, G. L., \& Roshko, A. (1974). "On density effects and large structure in turbulent mixing layers," J. Fluid Mech., 64(4), pp. 775-816.

[165] Gutmark, E. J., Schadow, K. C. \& Yu, K. H. (1995). "Mixing enhancement in supersonic free shear flows," Annu. Rev. Fluid Mech., 27(1), pp. 375-417.

[166] Michalke, A. (1965). "Vortex formation in a free boundary layer according to stability theory," J. Fluid Mech., 22(2), pp. 321-383.

[167] Karimi, M. (2015). Compressibility effects on Kelvin-Helmholtz instability and mixing layer flows, PhD Thesis, Texas A\&M University.

[168] Samimy, M., Reeder, M. F., \& Elliott, G. S. (1992). "Compressibility effects on large structures in free shear flows," Phys. Fluids A: Fluid Dynamics, 4(6), pp. 1251-1258. 
[169] Birch, S. F., Eggers, J. M. (1973). "A critical review of the experimental data for developed free turbulent shear layers," NASA Paper.

[170] Munson, B. R., Okiishi, T. H., Huebsch, W. W., \& Rothmayer, A. P. (2013). Fundamentals of Fluid Mechanics, $7^{\text {th }}$ ed., John Wiley \& Sons.

[171] Benham, P. P., Crawford, R. J., \& Armstrong, C. G. (1996). Mechanics of Engineering Materials, $2^{\text {nd }}$ ed., Longman Limited. 


\section{Appendix A}

\section{Wind-Tunnel Calibration}

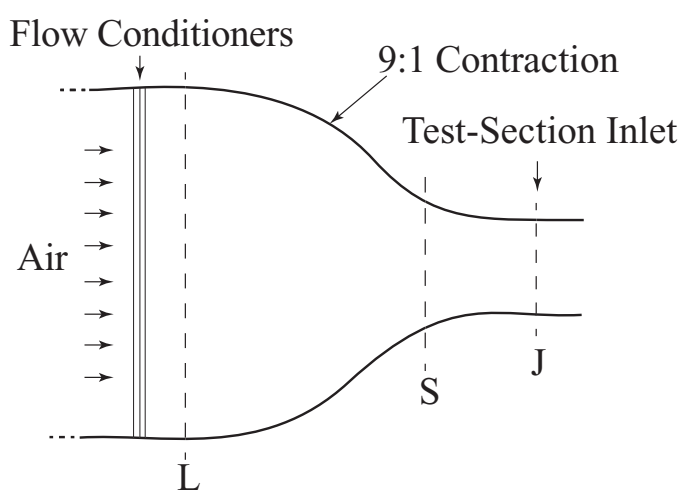

Figure A.1: Schematic of the wind-tunnel measurement stations

The contraction pressure difference measurement, $p_{L}-p_{S}$, is based on wall static pressures recorded at locations $\mathrm{L}$ and $\mathrm{S}$ in Figure A.1. Prior to the experiments with the bluff-body model, this pressure difference was correlated to the centerline dynamic pressure, $q_{J}$, measured with a Pitot static probe at the test section inlet (plane J in Figure A.1). Over the speed range considered, the correlation was linear, as illustrated in Figure A.2. The calibration was performed with an empty test section thereby enabling the calibration speed range to extend to a top speed of $55 \mathrm{~m} / \mathrm{s}$.

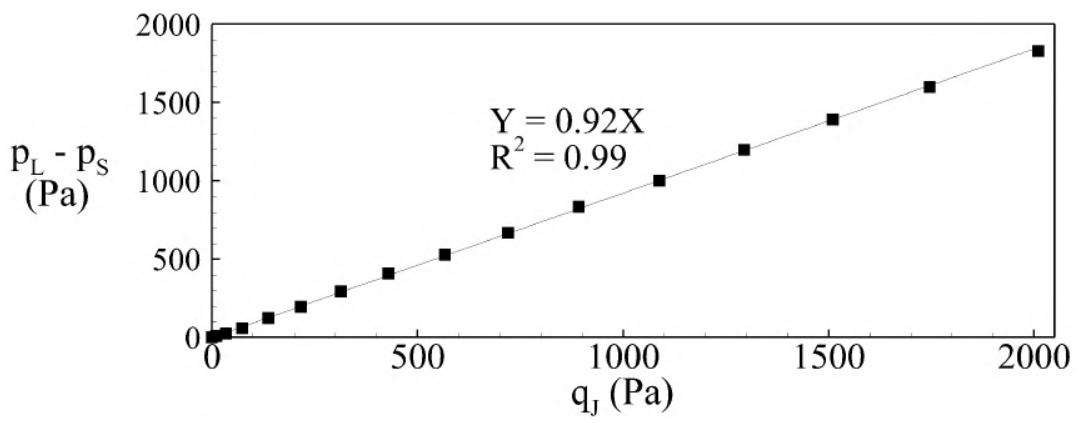

Figure A.2: Correlation of test-section inlet dynamic pressure to the contraction pressure difference 


\section{Appendix B}

\section{Prediction of the Wake Dynamics for the Baseline Configuration Using OpenFOAM}

This chapter presents the wake dynamics captured using the OpenFOAM, with a primary objective of demonstrating the consistency of the CFD results with those obtained from the CFX. This serves as a starting point for similar numerical problems where the CFX software can potentially be replaced by the OpenFOAM in the future.

\section{B.1 Computational Method}

An open-source computational fluid dynamics (CFD) software, OpenFOAM (version 4.1), was used to solve the incompressible form of the time-varying Reynoldsaveraged Navier-Stokes (RANS) equations together with a turbulence closure as described in Section 5.2.1.

\section{B.1.1 Discretization}

In the solution algorithm utilized by OpenFOAM software, the mass conservation equation is modified by expressing the velocity appearing in this equation in terms of pressure via the momentum conservation equation, thereby yielding Poisson's equation governing the pressure distribution in the flow field.

The spatial discretization is based on the cell-centered finite volume technique, with the various spatial interpolations of the flow parameters from cell centers to the control volume surfaces realized in manners that are equivalent to second-order centered differencing of all spatial derivatives except for those in the convection term of the momentum equations which are approximated using second-order upwind differencing proposed by Beam and Warming [151]. Boundedness of the second-order

upwind differencing is realized via a cell-to-cell gradient limiter which avoids over- and 
under-shoots on the gradient computations. The interpolation technique of Rhie and Chow [145] is utilized for the pressure contributions to the velocity of the convection terms to prevent the decoupling of the velocity and pressure fields on non-staggered grids. The time derivatives are discretized via second-order backward differencing. The convection terms of the momentum equations are linearized using the Picard iteration technique.

\section{B.1.2 Solution Method}

The iterative coupled solution of the pressure and velocity fields is obtained based on a combination of the Pressure-Implicit-Split-Operator (PISO) algorithm and the Semi-Implicit-Method-of-Pressure-Linked-Equations (SIMPLE) algorithm. Conventionally, this blended algorithm is referred to by the acronym PIMPLE. Within each time step, the transport equations are solved using the following iterative procedure that is originally developed by Patankar [152]:

i The domain is initialized using the most up-to-date pressure $(p)$, velocity $\left(U_{x}, U_{y}, U_{z}\right)$ and turbulence $(k, \omega)$ fields. The system of algebraic equations resulting from the discretization process are assembled in the form of $A \phi=b$, where $\mathrm{A}$ is a sparse coefficient matrix, $\phi$ is the vector of dependent variables, and $b$ is the forcing vector of source terms.

ii The coefficients in A are computed using the most recent values for the flow parameters.

iii The $\mathrm{x}, \mathrm{y}$, and $\mathrm{z}$ momentum equations are solved sequentially using an iterative solver to obtain to update the velocity field.

iv This velocity field is under-relaxed. Steps ii to iv are repeated until a userspecified iteration count is reached.

$\mathrm{v}$ The coefficients in matrix A are updated.

vi The correction to the pressure field is solved through the pressure equation using the most recent velocity field. 
vii The pressure-correction is under-relaxed.

viii The velocity field is updated based on the computed corrections to the pressure field, and steps ii to viii are repeated until convergence.

ix The velocity field is updated through explicit solution of the momentum equations.

$\mathrm{x}$ The pressure-correction equation is solved to update the velocity and the pressure fields. Steps ix to $\mathrm{x}$ are repeated for a specified iteration count.

xi The turbulence transport equations are solved based on the most recent pressure and velocity fields.

xii Steps ii to xi are repeated until the desired residual thresholds are reached.

xiii The solution is advanced in time and the same procedure is repeated.

The linear system of discretized momentum and turbulence transport equations are solved using the Gauss-Seidel iterative method. The pressure-correction equation based on mass conservation is solved through V-type algebraic multigrid cycles. Twelve grid levels, determined after an initial simulation to optimize the rate of convergence, are used in the restriction and prolongation legs with three Gauss-Seidel smoothing iterations performed per grid level in both restriction and prolongation legs of the multi-grid cycle. At the coarsest grid level, the solution is obtained using a preconditioned bi-conjugate gradient solver.

In iterative updating of the flow variables, an under-relaxation factor of 0.3 is applied to the pressure field while a factor of 0.7 is used for the velocity and the turbulence fields. In each time step, iterative solution of the discretized governing equations is declared converged upon reducing the normalized residuals of the governing equations to less than $1 \times 10^{-6}$.

Figure B.1 presents the comparison of the wake structures captured via the OpenFOAM and CFX. The OpenFOAM algorithm has been successful in capturing the $\mathrm{K}-\mathrm{H}$ instability vortices and helical pattern, with a relatively higher numerical diffusion observed farther downstream of the wake compared to that of the CFX case. 
a)

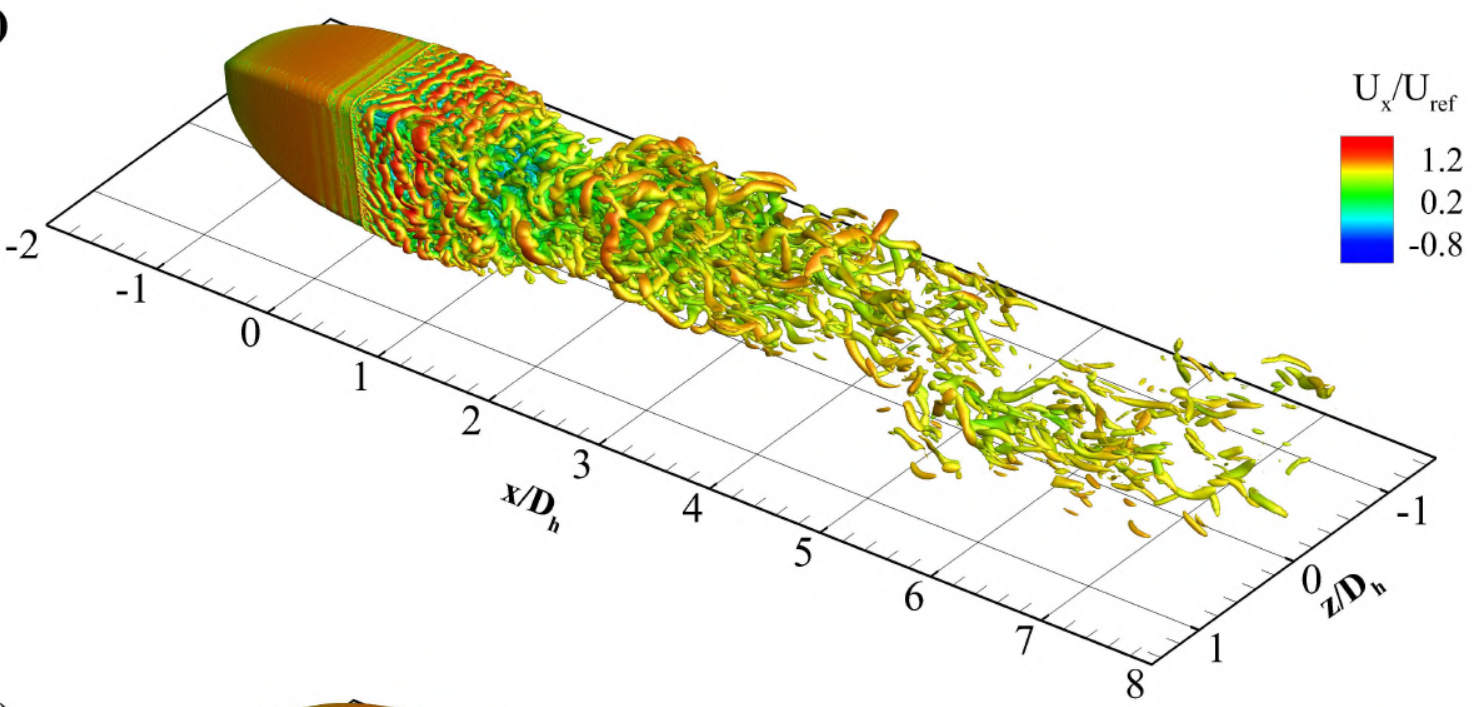

b)

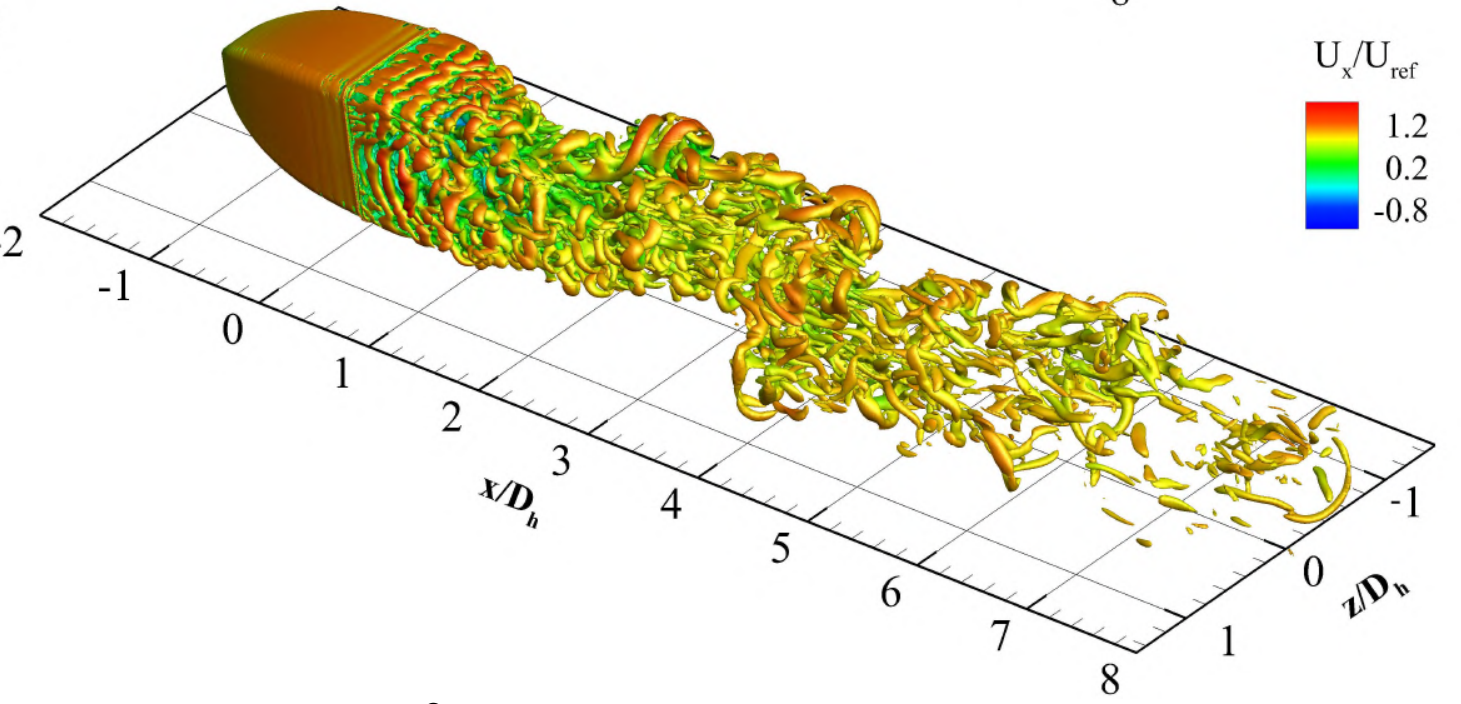

Figure B.1: Q $/\left(\mathrm{U}_{\text {ref }} / \mathrm{D}_{\mathrm{h}}\right)^{2}=9.5$ isosurfaces in a) OpenFOAM, b) CFX for the baseline configuration in the absence of small-scale turbulence

As the same spatial grid is used for both cases, this difference is attributed to the discretization scheme used for the convection term of the momentum equations that is equivalent to second-order upwind differencing in the OpenFOAM. A hybrid of first-order upwind and second-order centered differencing is used in the CFX for the convection term. The difference in numerical diffusion can be avoided by increasing the spatial resolution in regions where the diffusion is undesired. The results promise a good starting point for the similar problems to be dealt with the OpenFOAM in the future. 


\section{Appendix C}

\section{Supplementary Plots on the Computational Results}

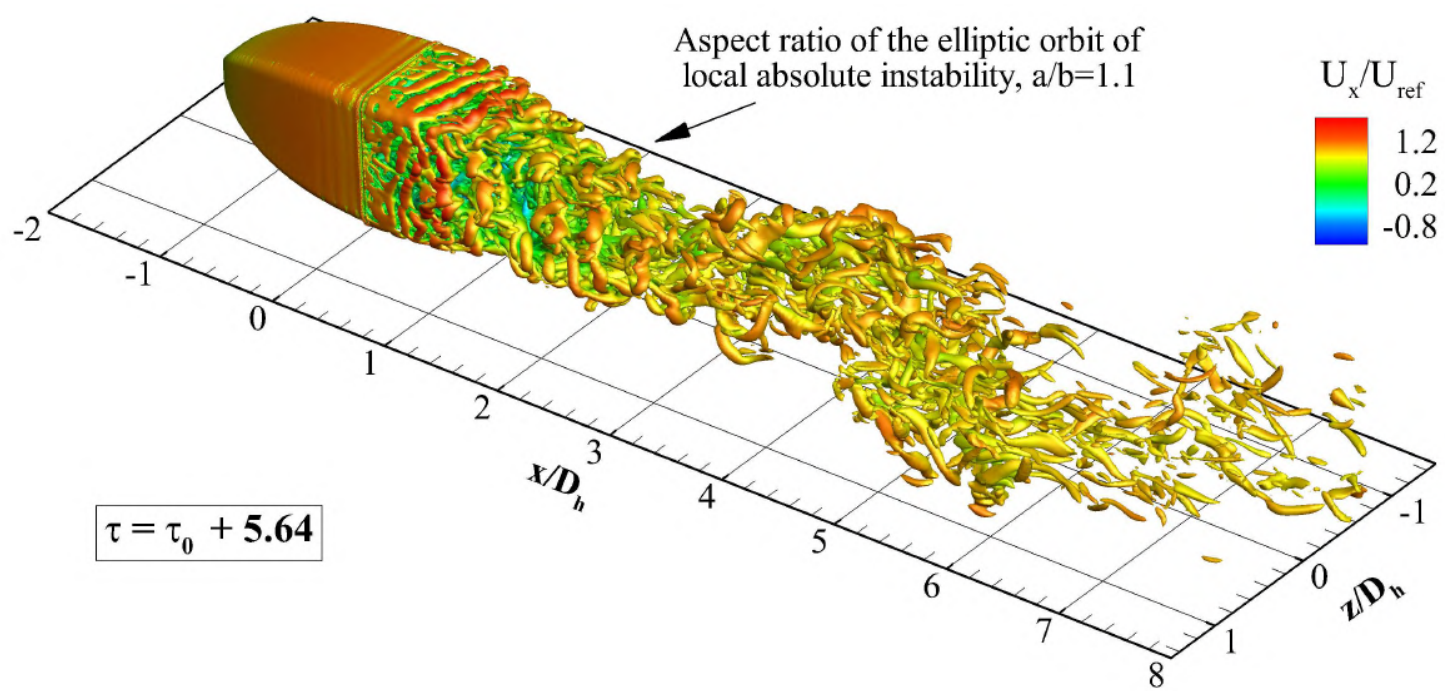

Figure C.1: $\mathrm{Q} /\left(\mathrm{U}_{\text {ref }} / \mathrm{D}_{\mathrm{h}}\right)^{2}=9.5$ isosurfaces in the baseline configuration in the absence of small-scale turbulence 


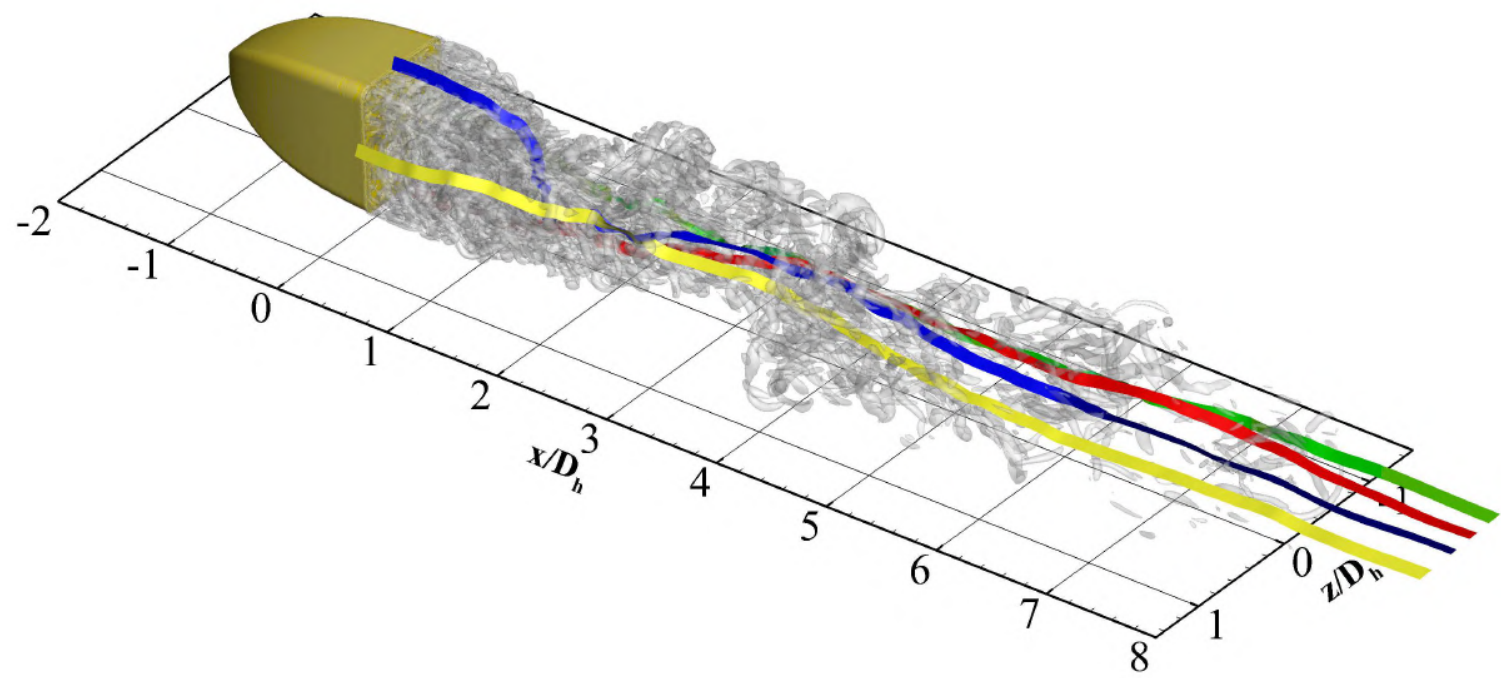

Figure C.2: Streamtrace ribbons with transculent isosurfaces of $\mathrm{Q} /\left(\mathrm{U}_{\text {ref }} / \mathrm{D}_{\mathrm{h}}\right)^{2}=9.5$ in the absence of small-scale turbulence in the baseline configuration

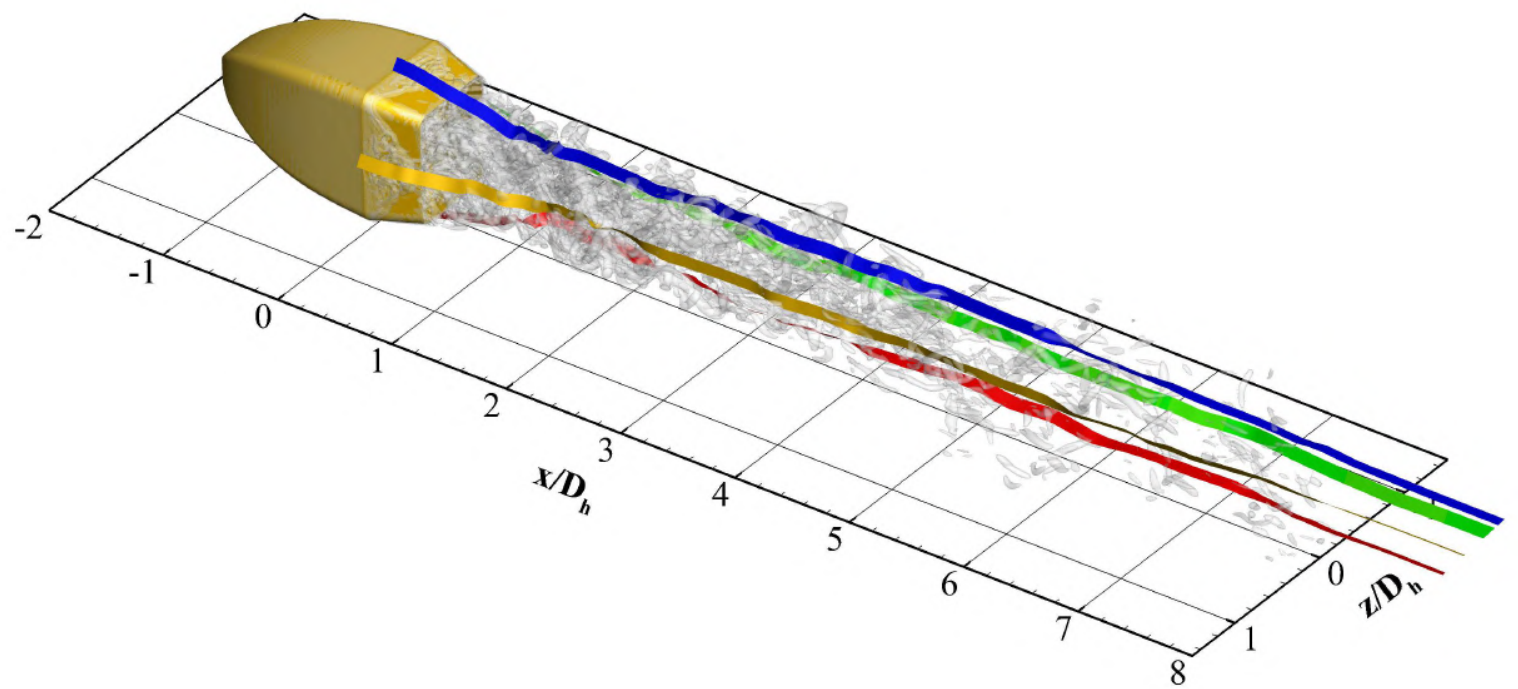

Figure C.3: Streamtrace ribbons with transculent isosurfaces of $\mathrm{Q} /\left(\mathrm{U}_{\text {ref }} / \mathrm{D}_{\mathrm{h}}\right)^{2}=9.5$ in the absence of small-scale turbulence in the straighttail configuration 


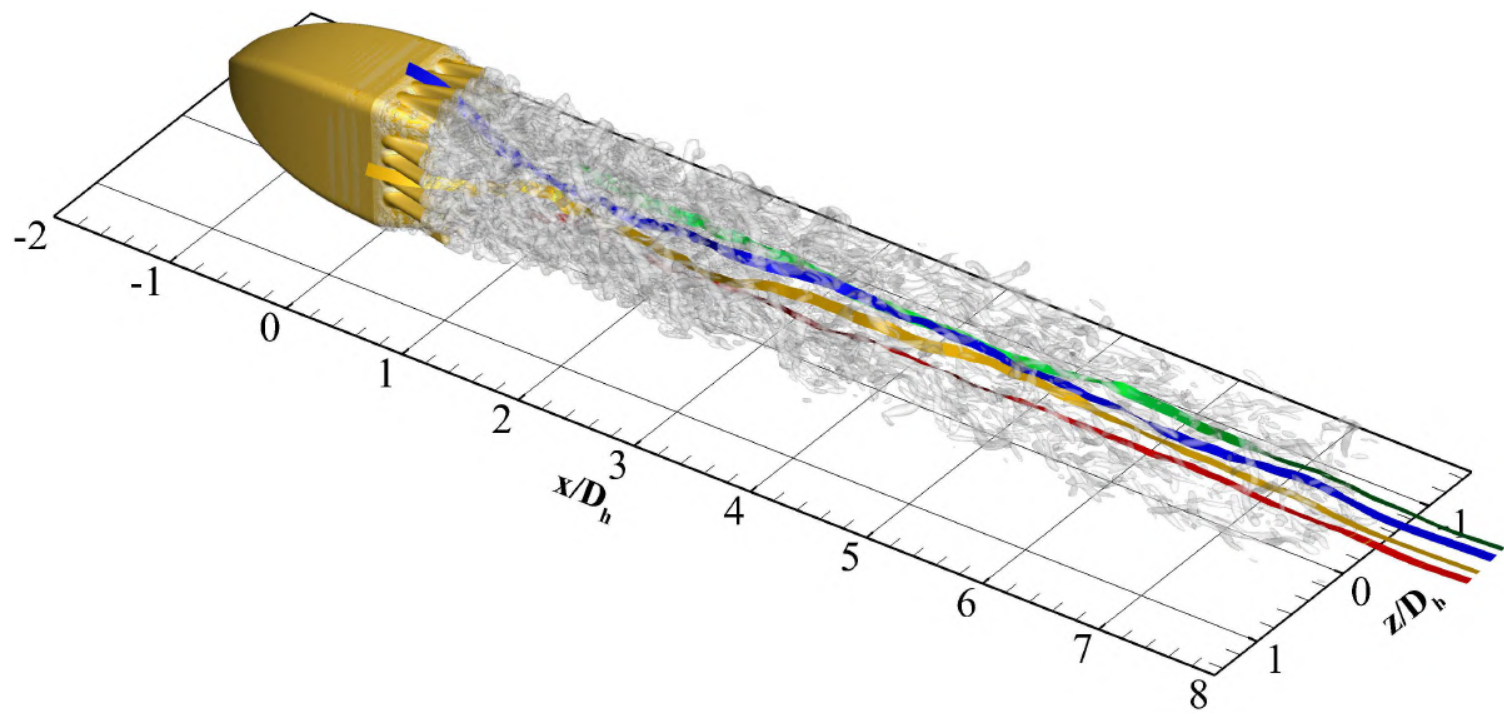

Figure C.4: Streamtrace ribbons with transculent isosurfaces of $\mathrm{Q} /\left(\mathrm{U}_{\text {ref }} / \mathrm{D}_{\mathrm{h}}\right)^{2}=9.5$ in the absence of small-scale turbulence in the lobedtail configuration

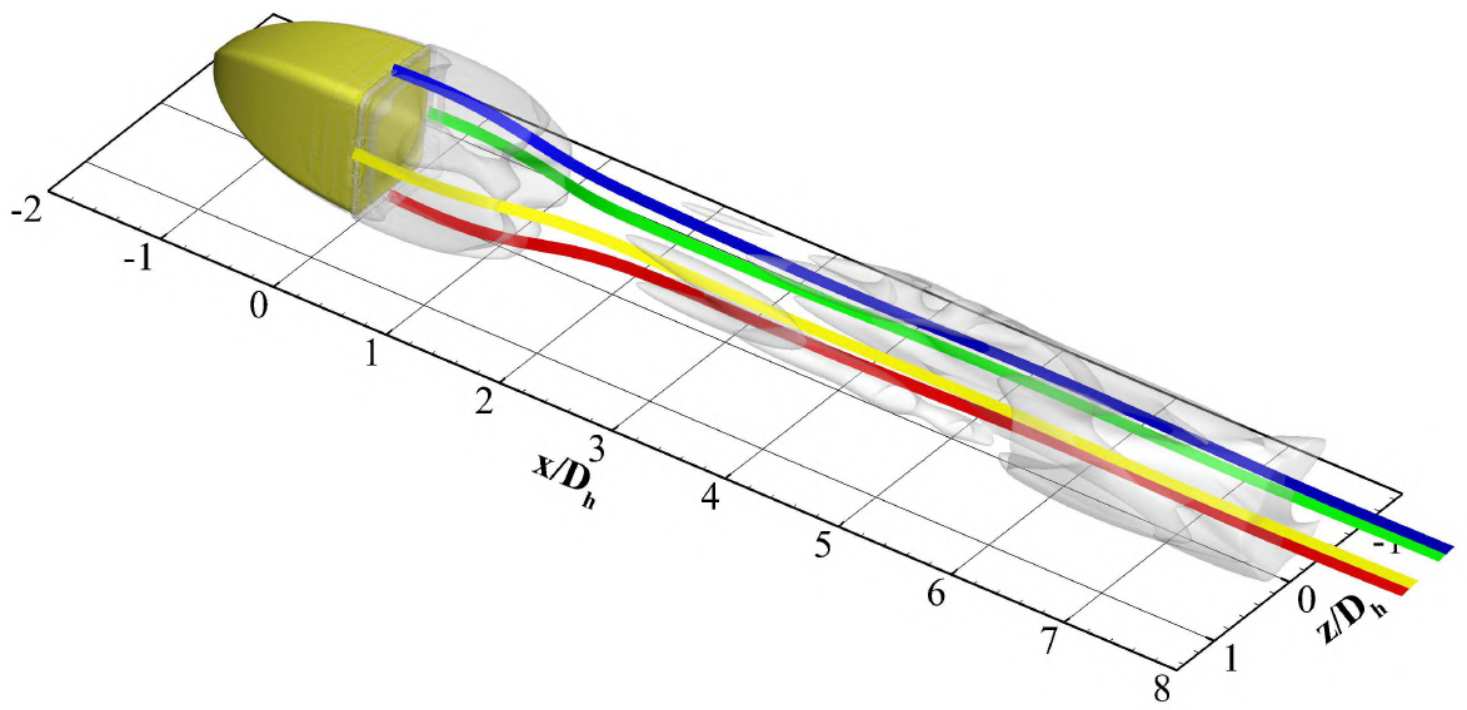

Figure C.5: Streamtrace ribbons with transculent isosurfaces of $\mathrm{Q} /\left(\mathrm{U}_{\text {ref }} / \mathrm{D}_{\mathrm{h}}\right)^{2}=1.2 \times 10^{-3}$ in the presence of small-scale turbulence in the baseline configuration 


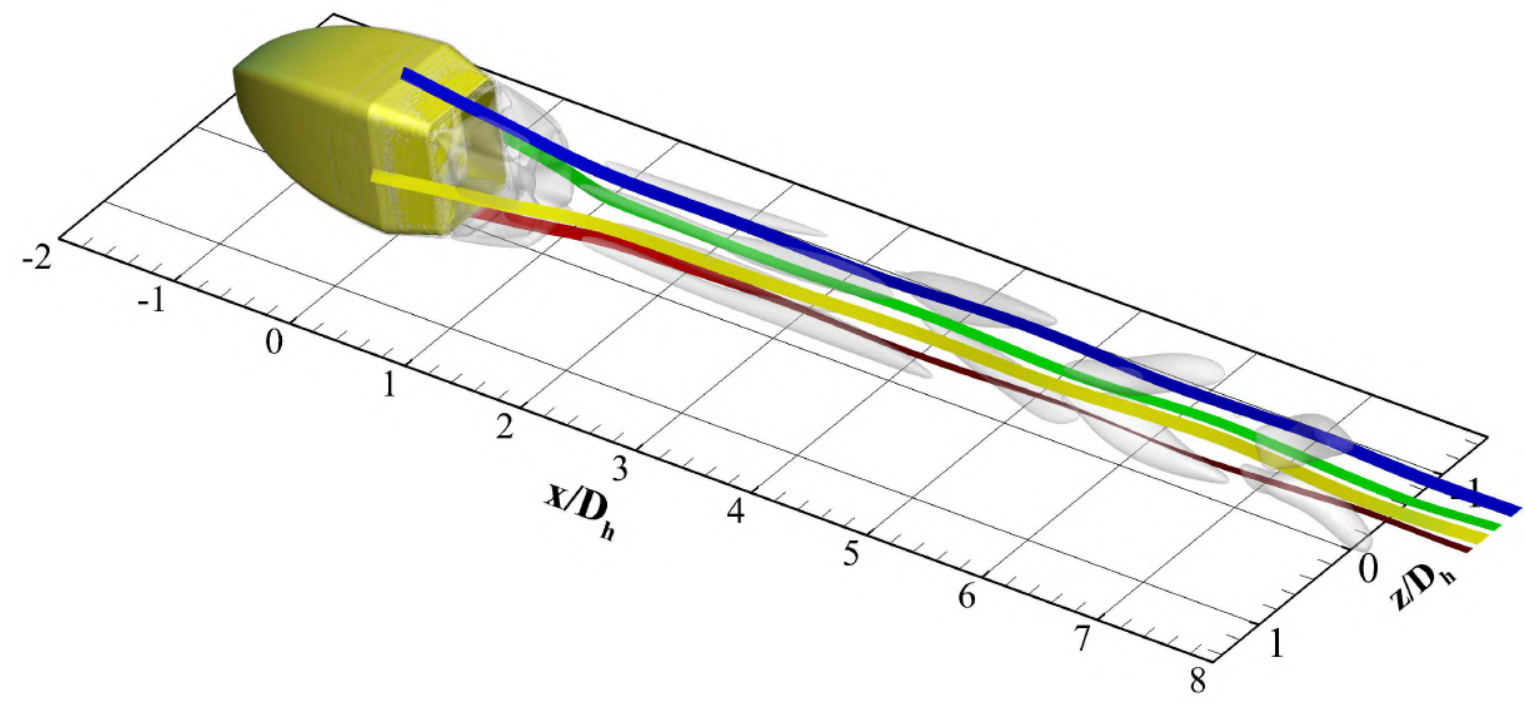

Figure C.6: Streamtrace ribbons with transculent isosurfaces of $\mathrm{Q} /\left(\mathrm{U}_{\text {ref }} / \mathrm{D}_{\mathrm{h}}\right)^{2}=1.25 \times 10^{-2}$ in the presence of small-scale turbulence in the straight-tail configuration

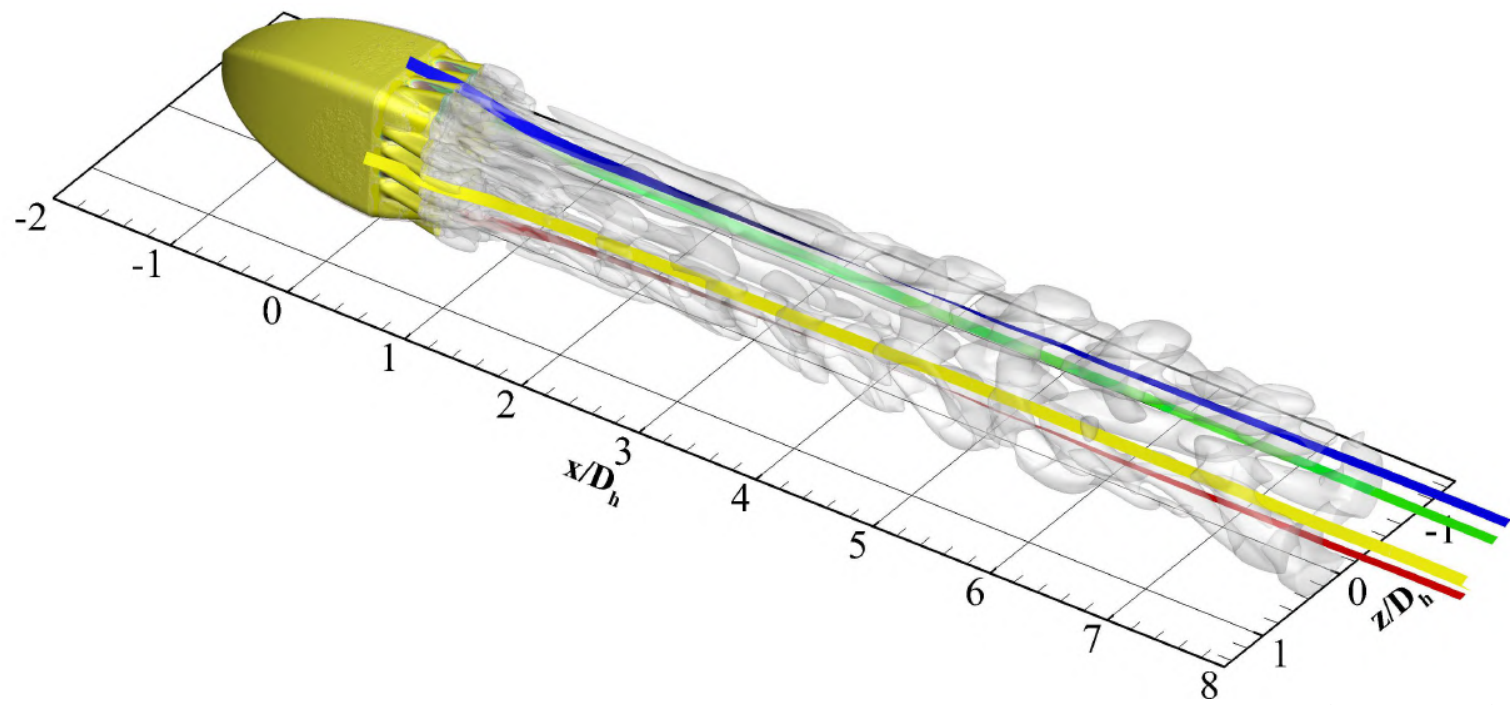

Figure C.7: Streamtrace ribbons with transculent isosurfaces of $\mathrm{Q} /\left(\mathrm{U}_{\mathrm{ref}} / \mathrm{D}_{\mathrm{h}}\right)^{2}=2.4 \times 10^{-2}$ in the presence of small-scale turbulence in the lobed-tail configuration 
a)

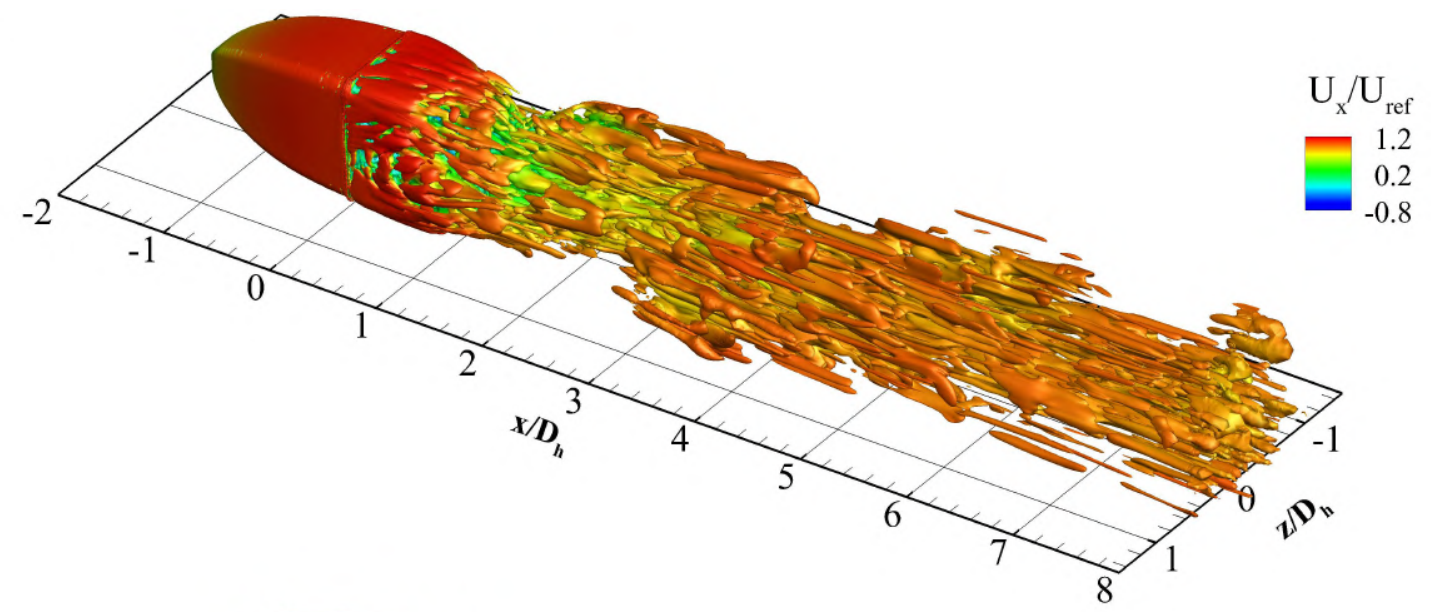

b)

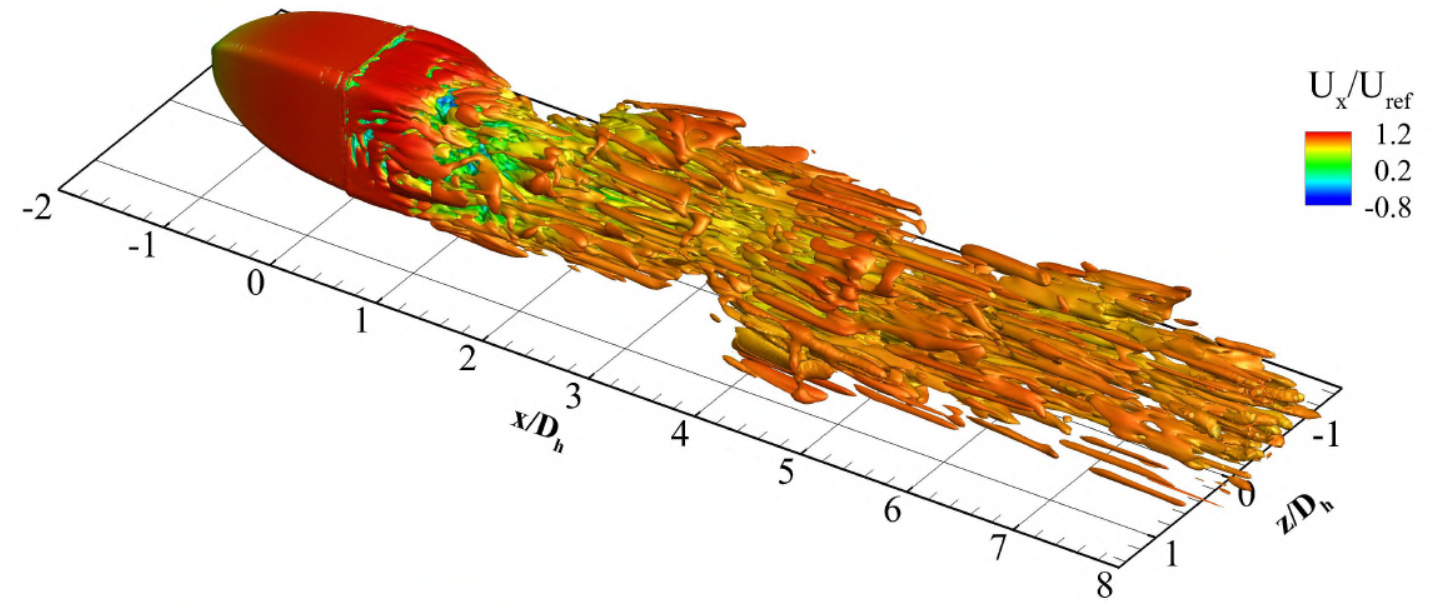

c)

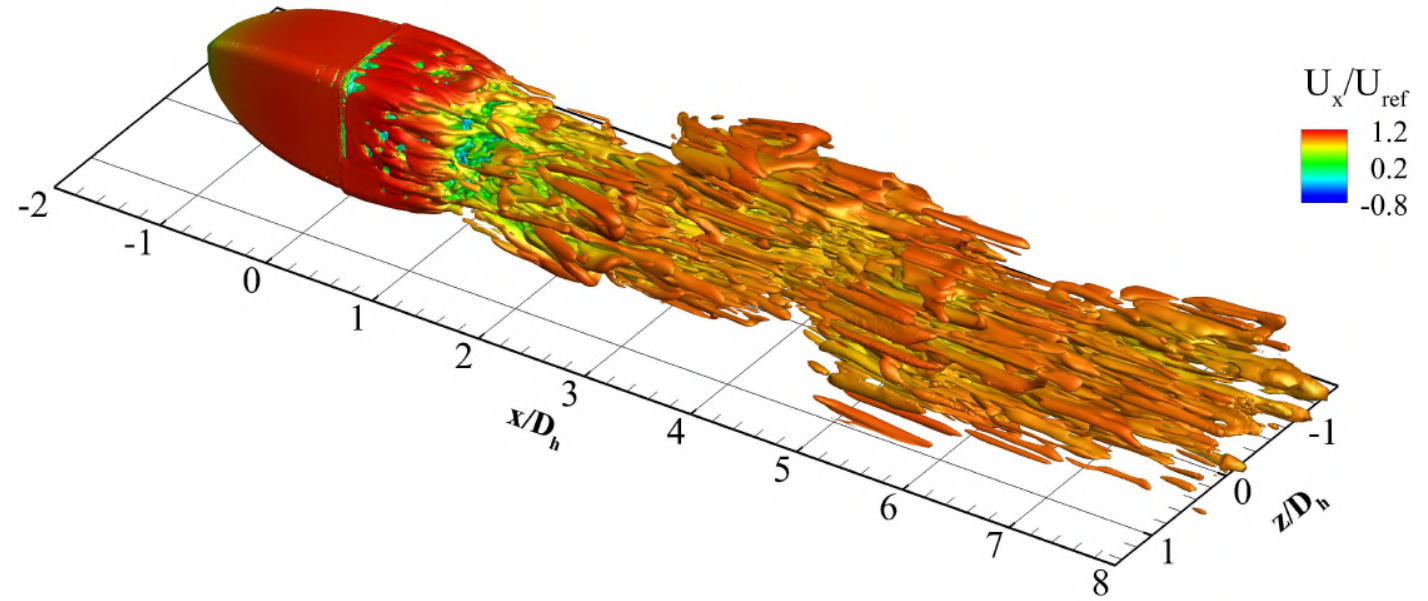

Figure C.8: $\mathrm{Q} /\left(\mathrm{U}_{\text {ref }} / \mathrm{D}_{\mathrm{h}}\right)^{2}=2.4 \times 10^{-1}$ isosurfaces calculated based on sequentially time-averaged flow fields with an averaging period of $10 \mathrm{~K}-\mathrm{H}$ cycles in the baseline configuration in the absence of small-scale turbulence 
a)

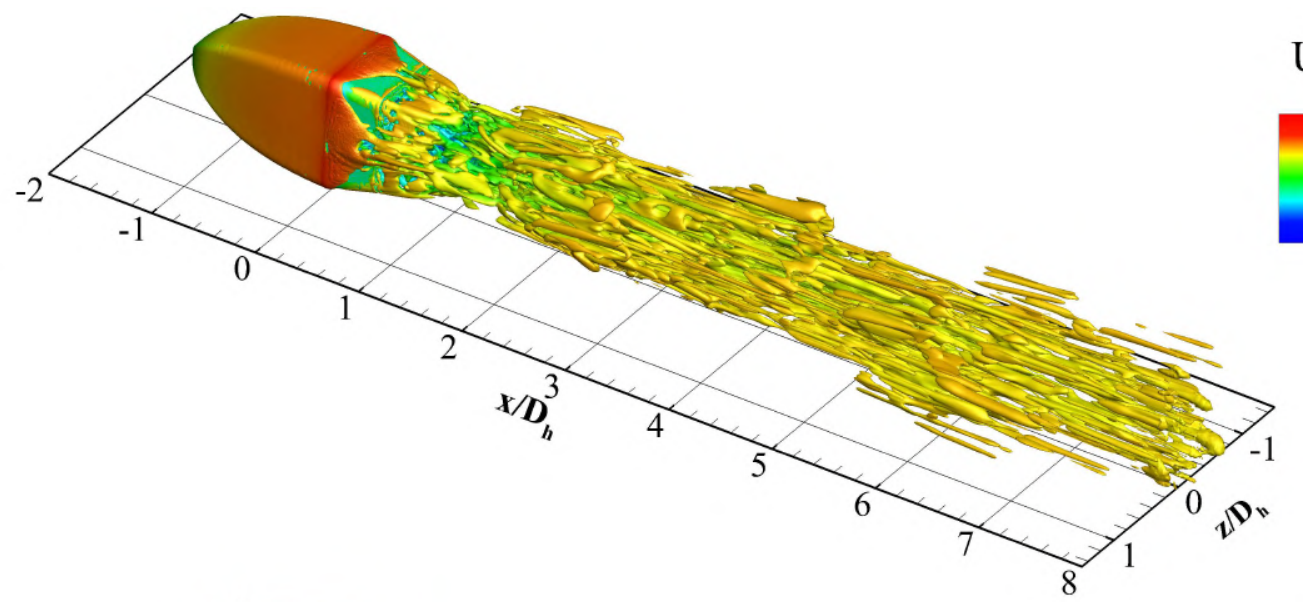

$\mathrm{U}_{\mathrm{x}} / \mathrm{U}_{\text {ref }}$

1.3

0.4

$-0.5$

b)

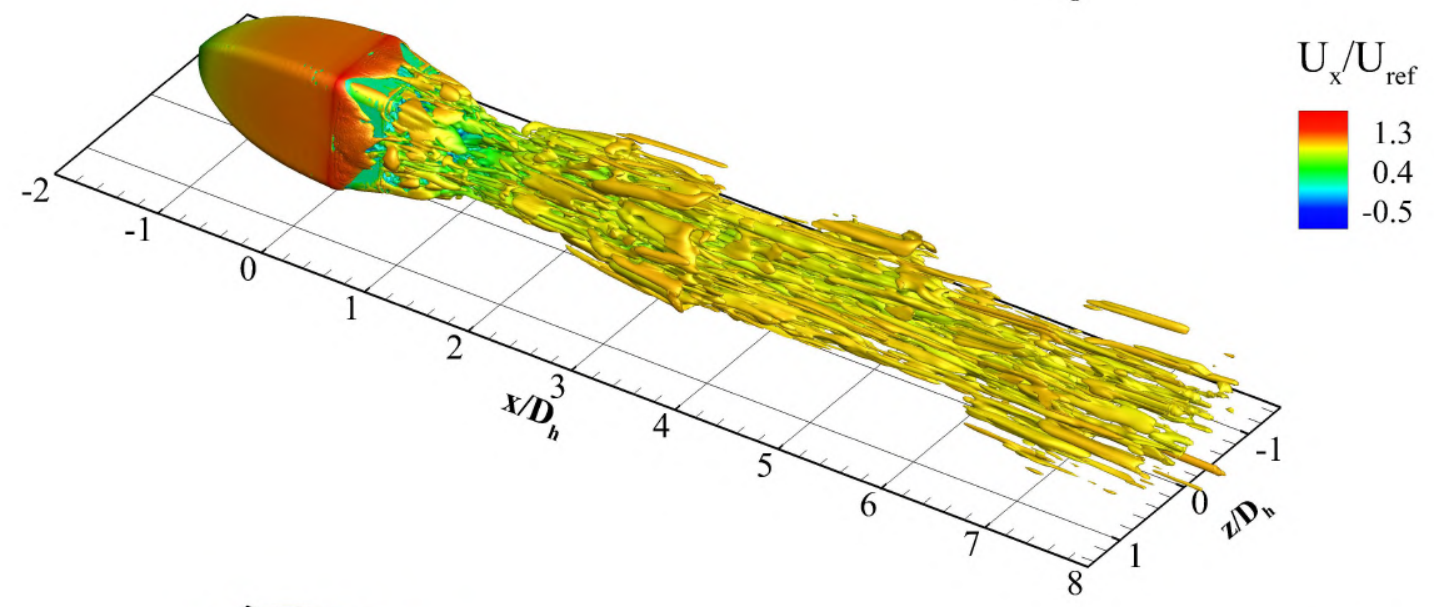

c)

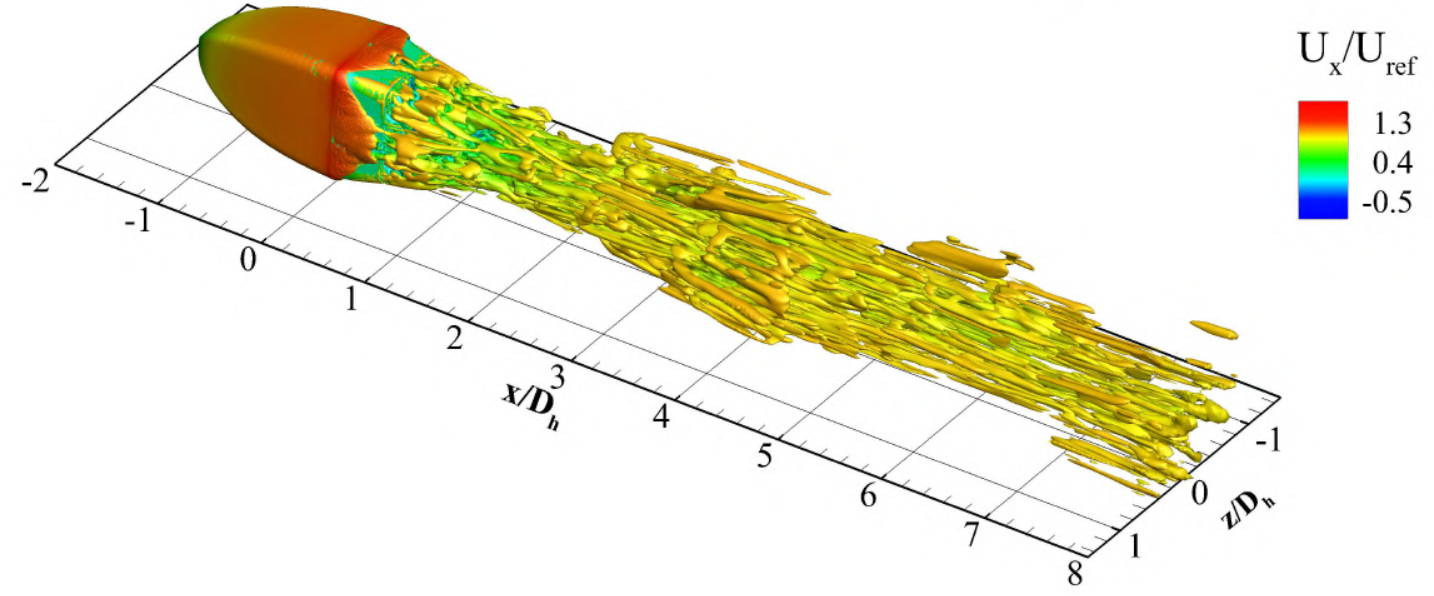

Figure C.9: $\mathrm{Q} /\left(\mathrm{U}_{\text {ref }} / \mathrm{D}_{\mathrm{h}}\right)^{2}=2.4 \times 10^{-1}$ isosurfaces calculated based on sequentially time-averaged flow fields with an averaging period of $10 \mathrm{~K}-\mathrm{H}$ cycles in the straight-tail configuration in the absence of small-scale turbulence 
a)

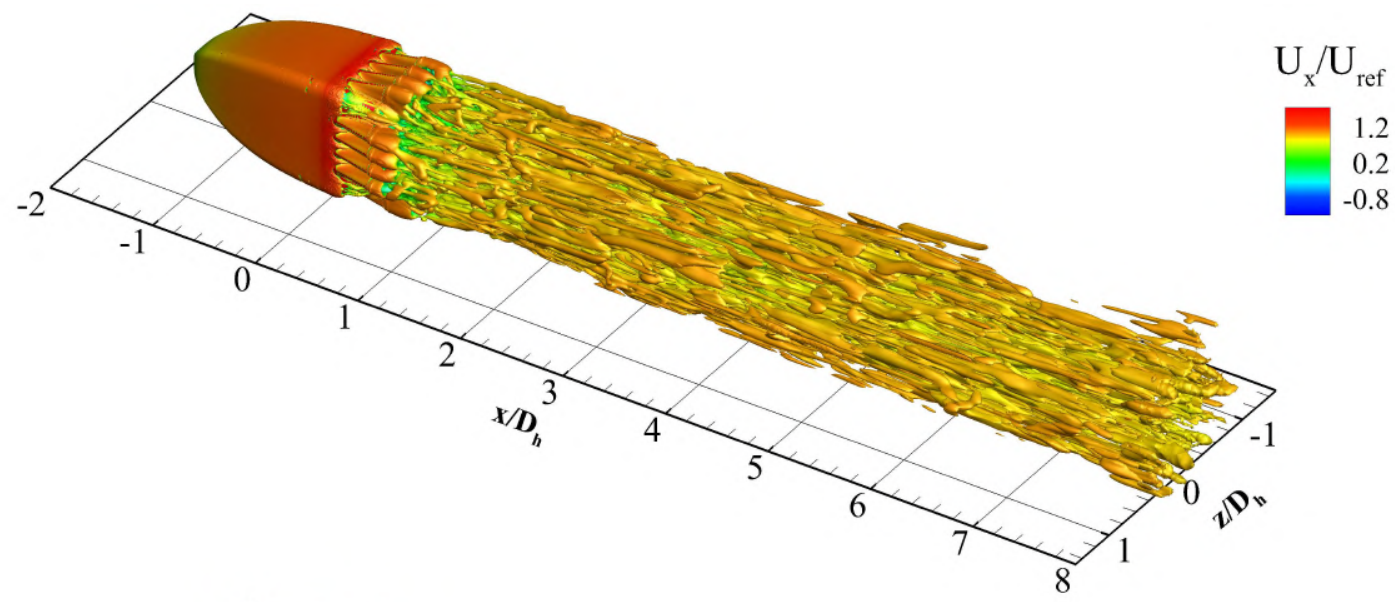

b)

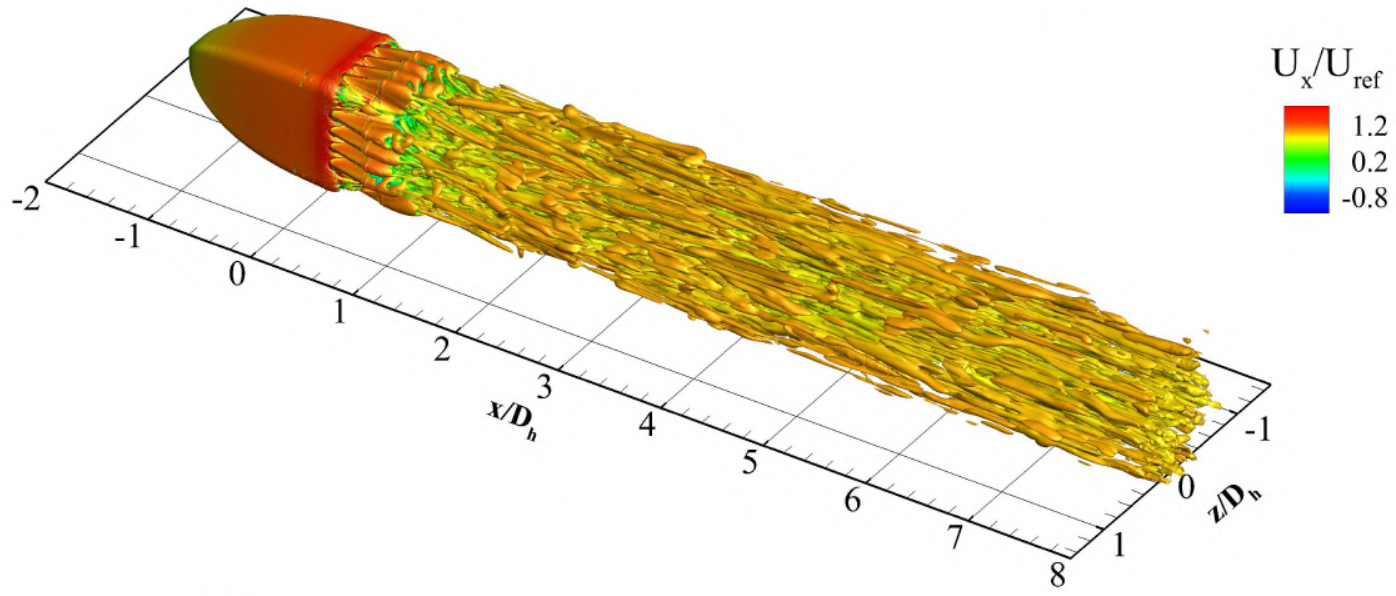

c)

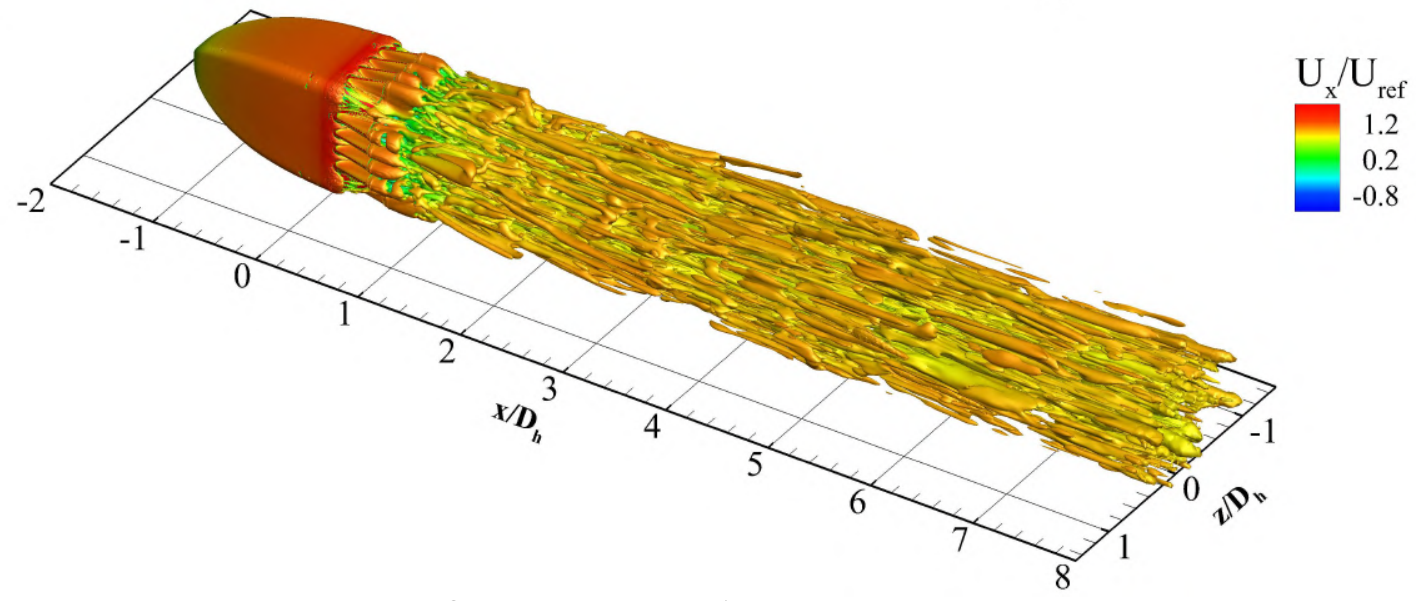

Figure C.10: $\mathrm{Q} /\left(\mathrm{U}_{\mathrm{ref}} / \mathrm{D}_{\mathrm{h}}\right)^{2}=2.4 \times 10^{-1}$ isosurfaces calculated based on sequentially time-averaged flow fields with an averaging period of $10 \mathrm{~K}-\mathrm{H}$ cycles in the lobed-tail configuration in the absence of small-scale turbulence 
a)

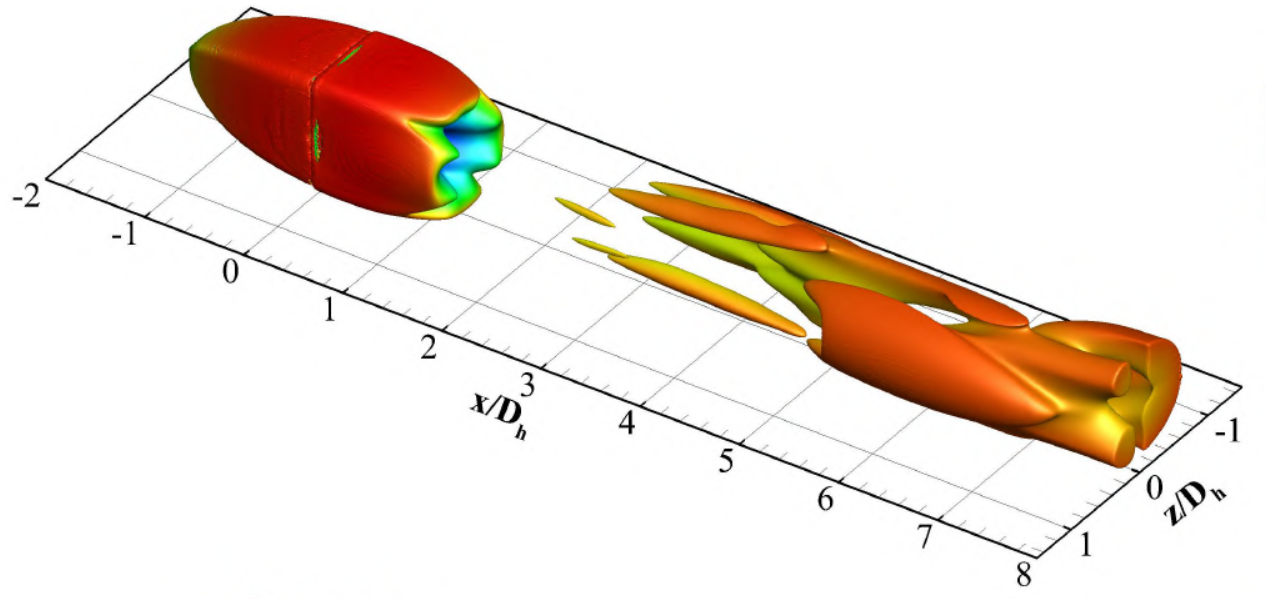

$\mathrm{U}_{\mathrm{x}} / \mathrm{U}_{\text {ref }}$

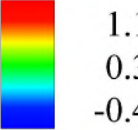

b)

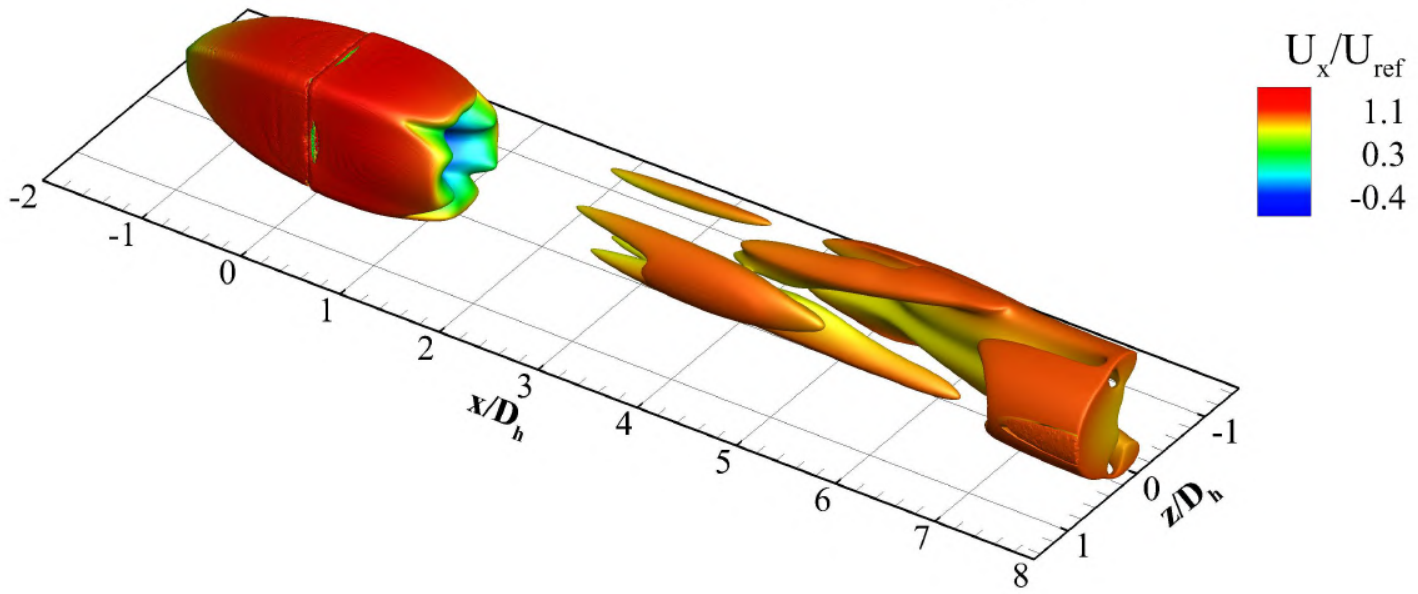

Figure C.11: $\mathrm{Q} /\left(\mathrm{U}_{\mathrm{ref}} / \mathrm{D}_{\mathrm{h}}\right)^{2}=2.4 \times 10^{-1}$ isosurfaces calculated based on sequentially time-averaged flow fields with an averaging period of $10 \mathrm{~K}-\mathrm{H}$ cycles in the baseline configuration in the presence of small-scale turbulence 
a)

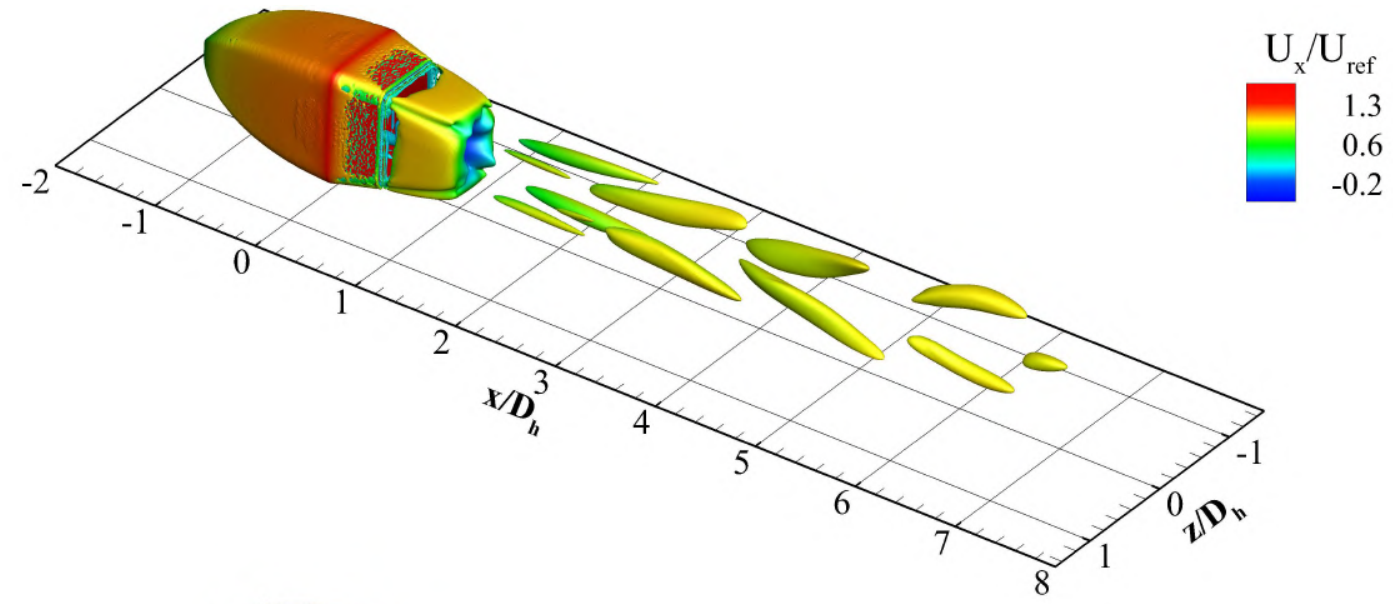

b)

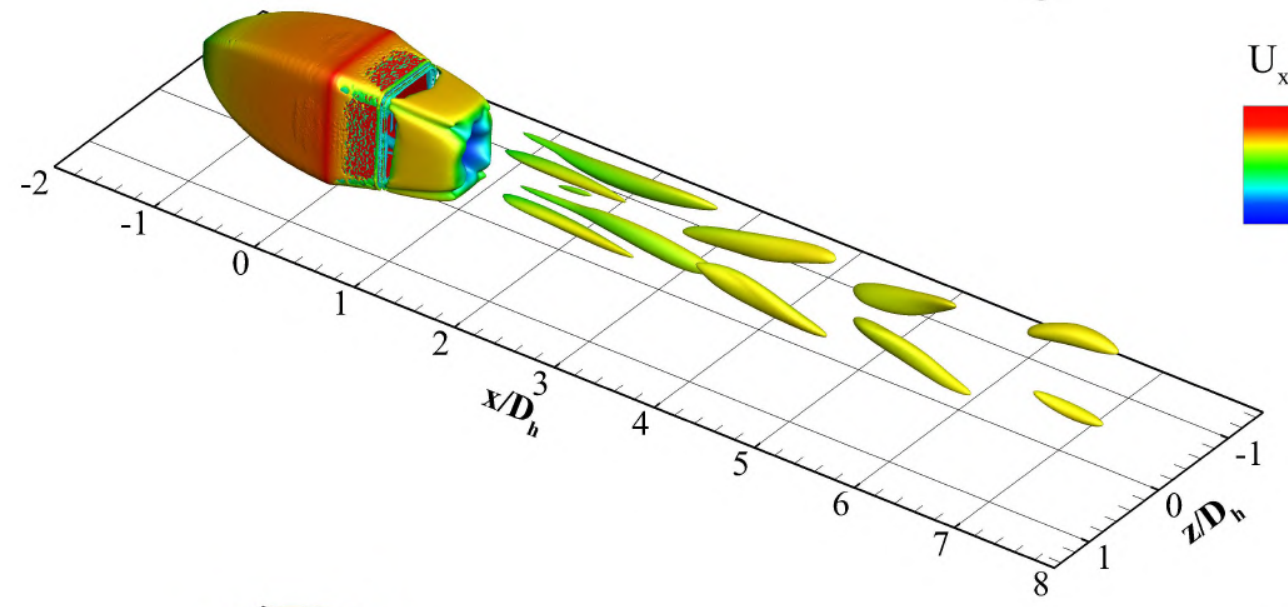

c)

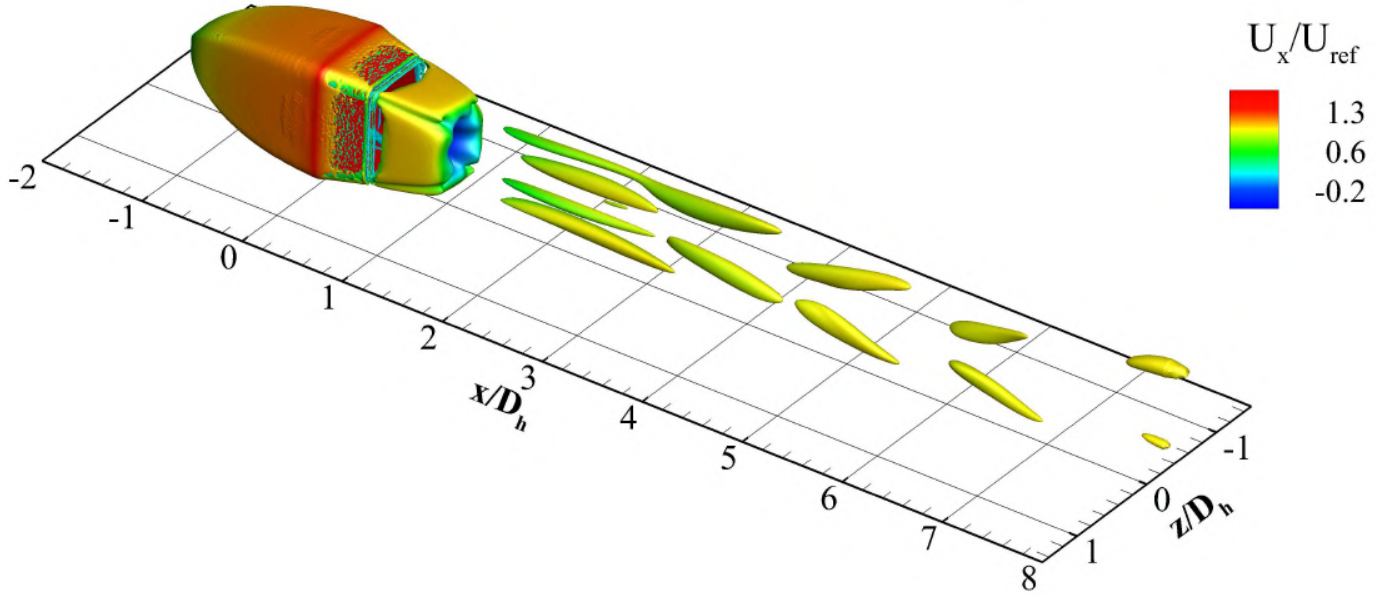

Figure C.12: $\mathrm{Q} /\left(\mathrm{U}_{\mathrm{ref}} / \mathrm{D}_{\mathrm{h}}\right)^{2}=2.4 \times 10^{-1}$ isosurfaces calculated based on sequentially time-averaged flow fields with an averaging period of $10 \mathrm{~K}$ $\mathbf{H}$ cycles in the straight-tail configuration in the presence of small-scale turbulence 
a)

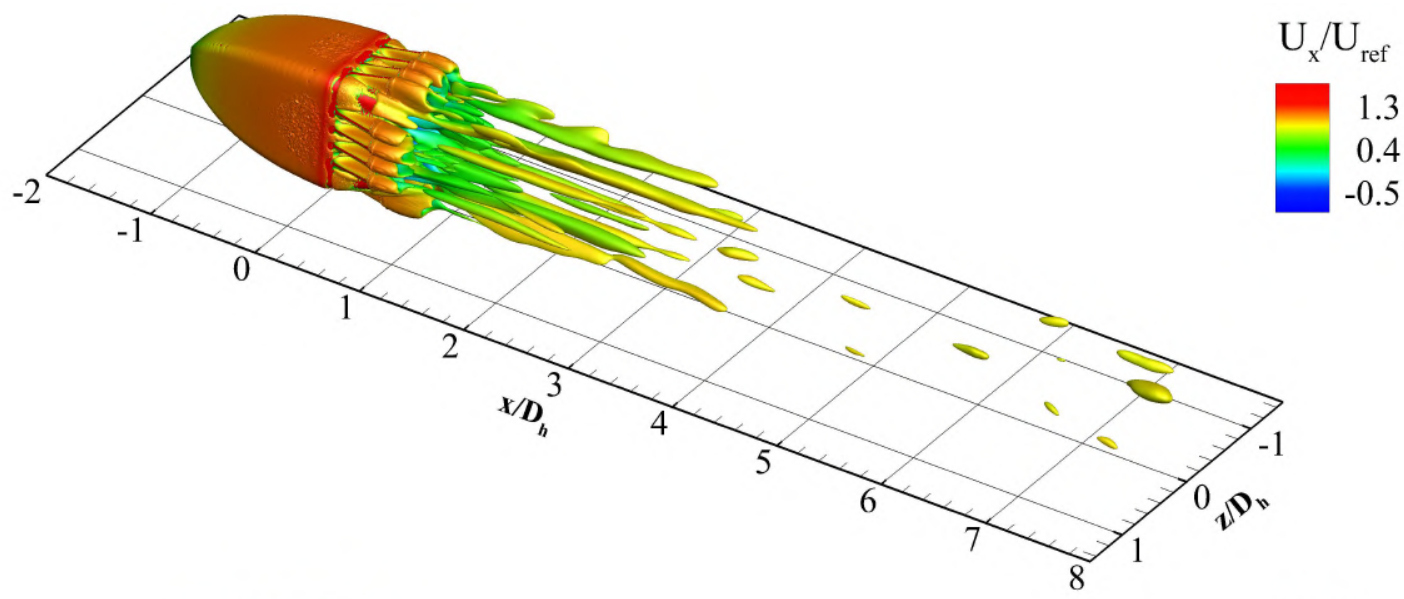

1.3

0.4

$-0.5$

b)

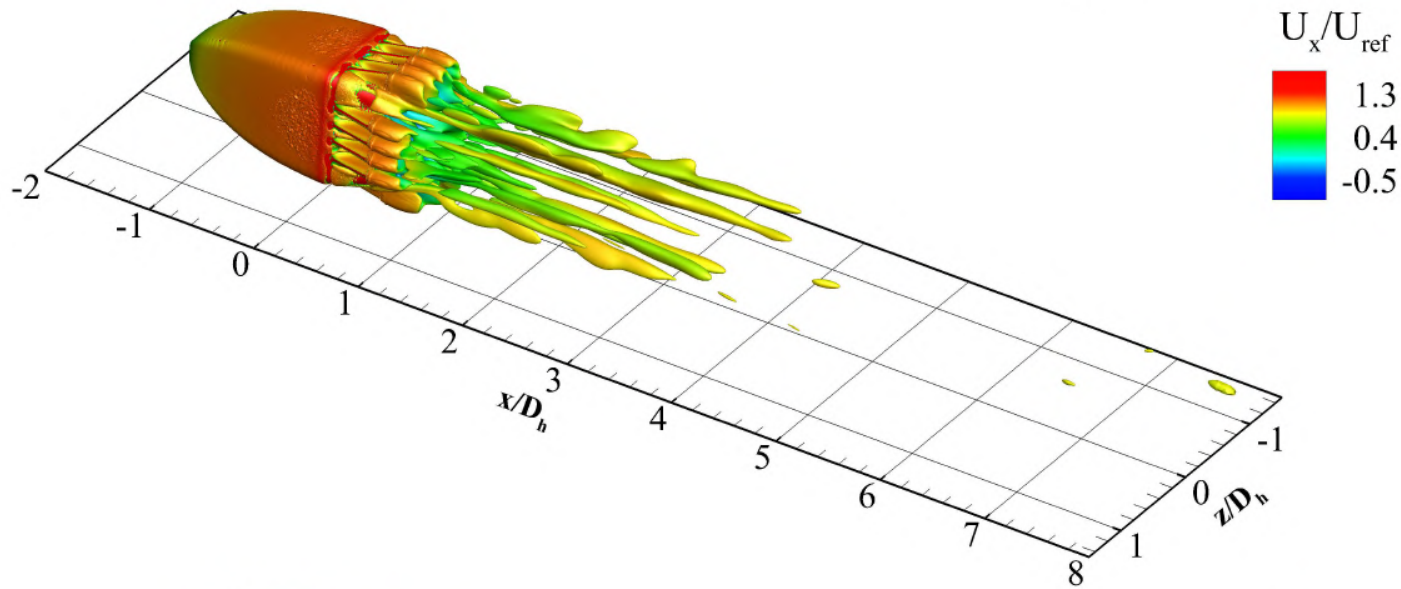

c)

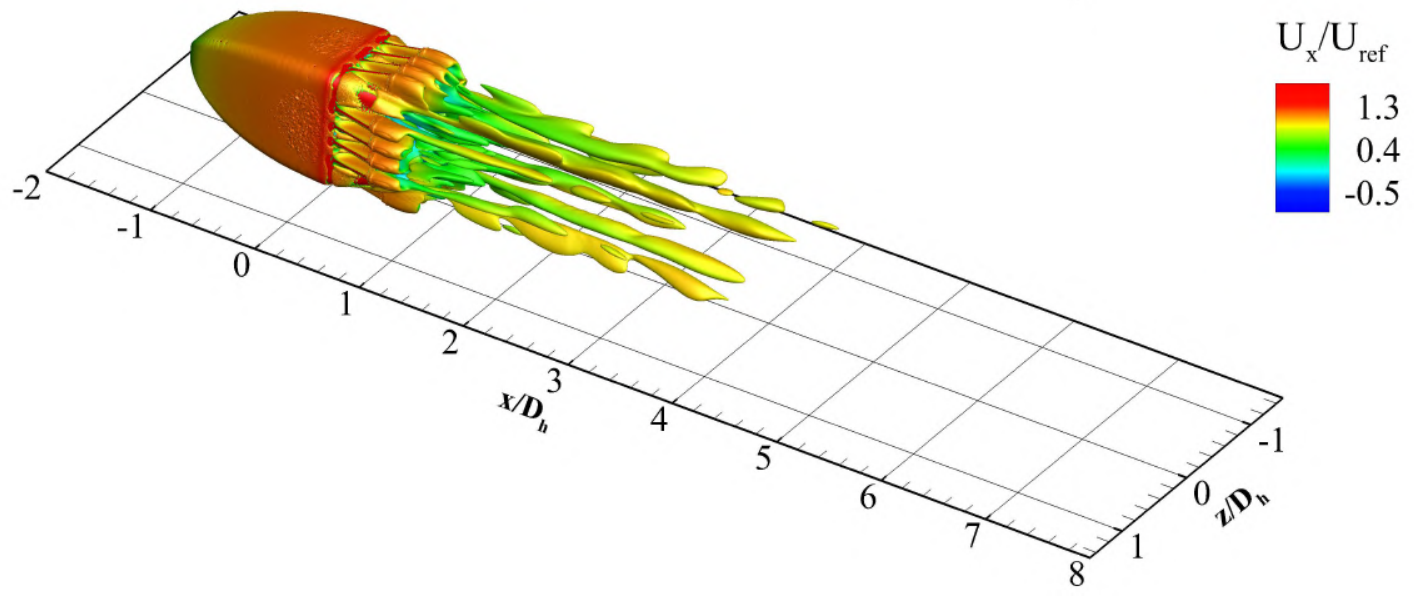

Figure C.13: $\mathrm{Q} /\left(\mathrm{U}_{\mathrm{ref}} / \mathrm{D}_{\mathrm{h}}\right)^{2}=2.4 \times 10^{-1}$ isosurfaces calculated based on sequentially time-averaged flow fields with an averaging period of $10 \mathrm{~K}-\mathrm{H}$ cycles in the lobed-tail configuration in the presence of small-scale turbulence 
a)

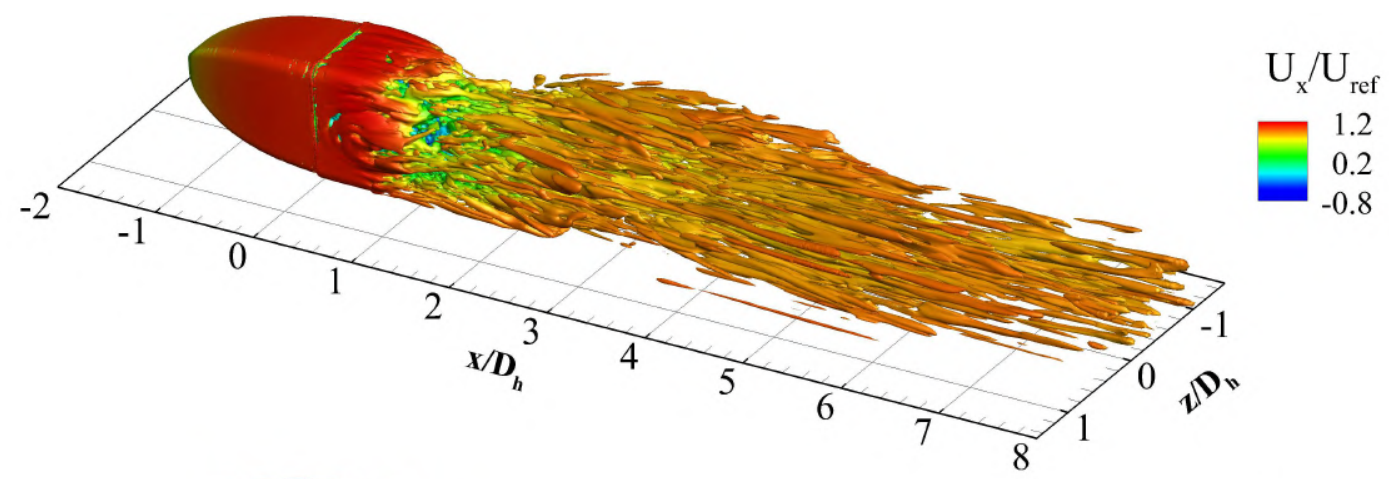

b)

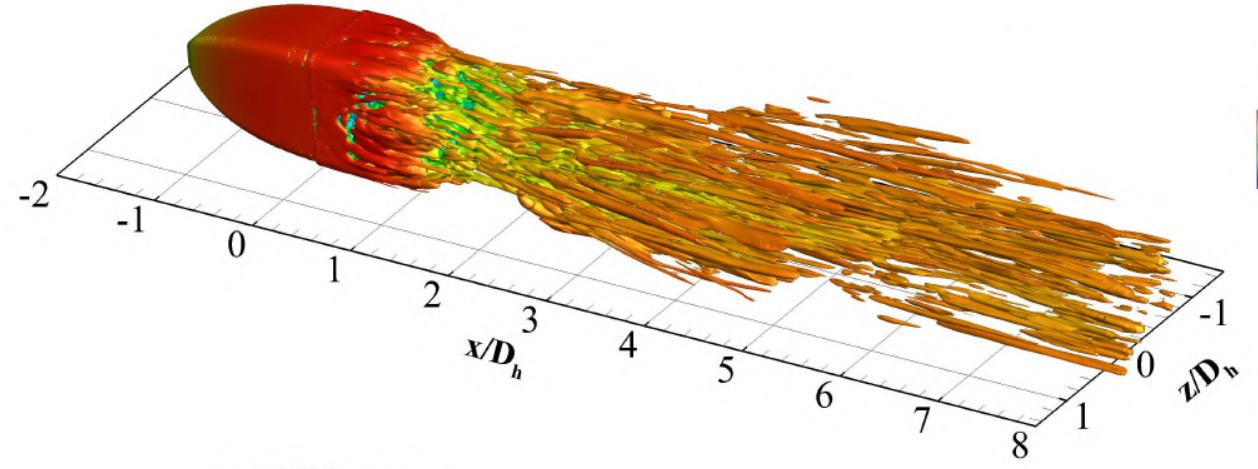

$\mathrm{U}_{\mathrm{x}} / \mathrm{U}_{\mathrm{ref}}$
1.2
0.2
-0.8

c)

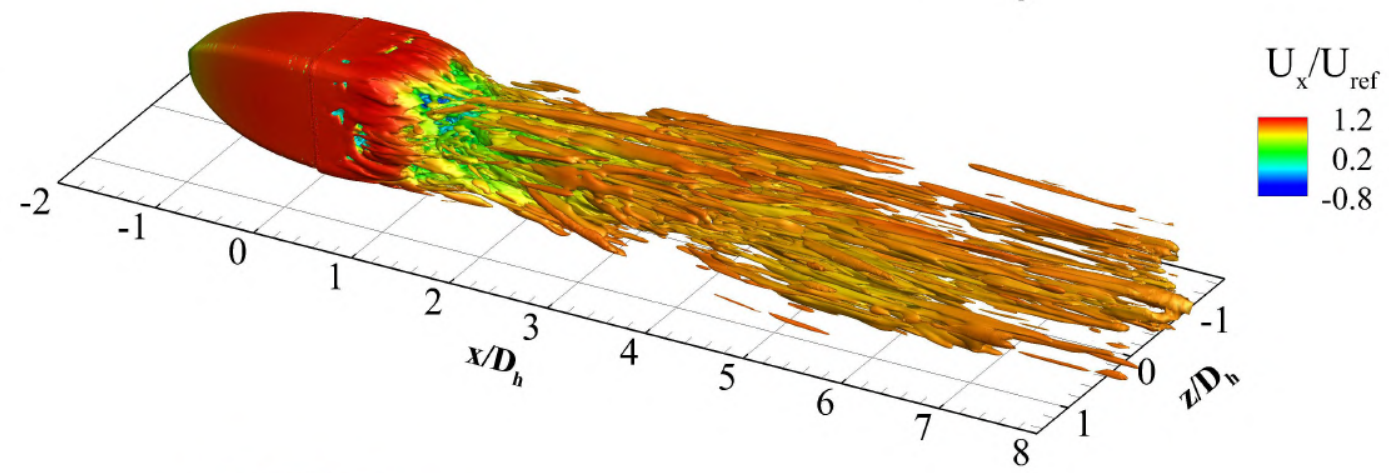

d)

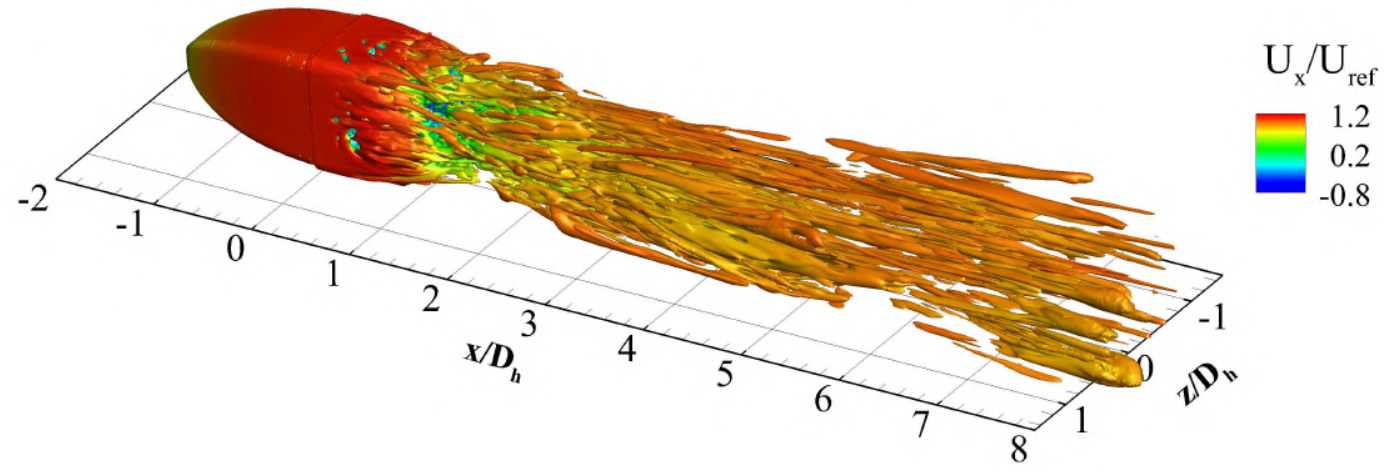

Figure C.14: $\mathrm{Q} /\left(\mathrm{U}_{\mathrm{ref}} / \mathrm{D}_{\mathrm{h}}\right)^{2}=2.4 \times 10^{-1}$ isosurfaces calculated based on sequentially time-averaged flow fields with an averaging period of $25 \mathrm{~K}-\mathrm{H}$ cycles in the baseline configuration in the absence of small-scale turbulence 
a)

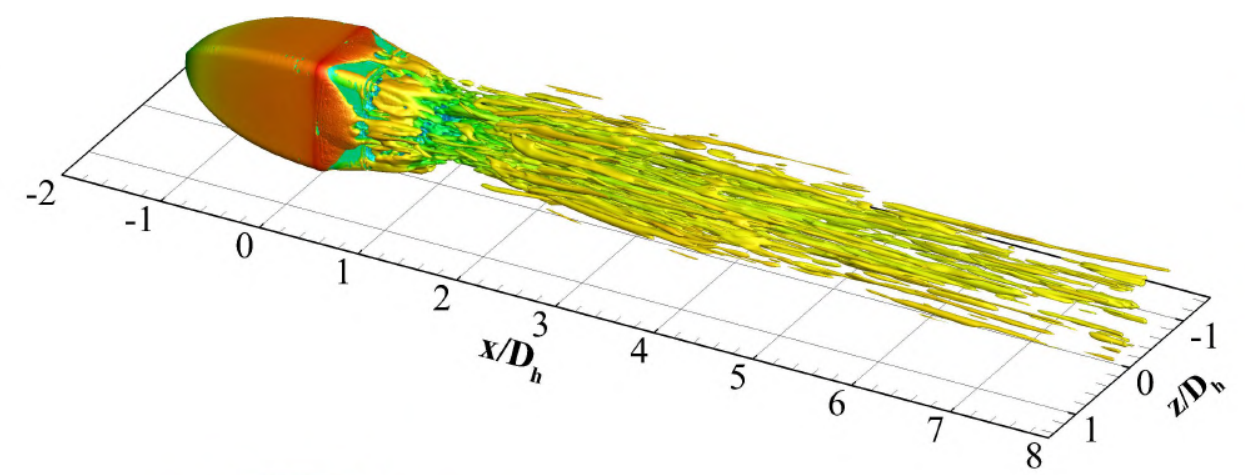

$\mathrm{U}_{\mathrm{x}} / \mathrm{U}_{\text {ref }}$

1.3

0.5

$-0.4$

b)

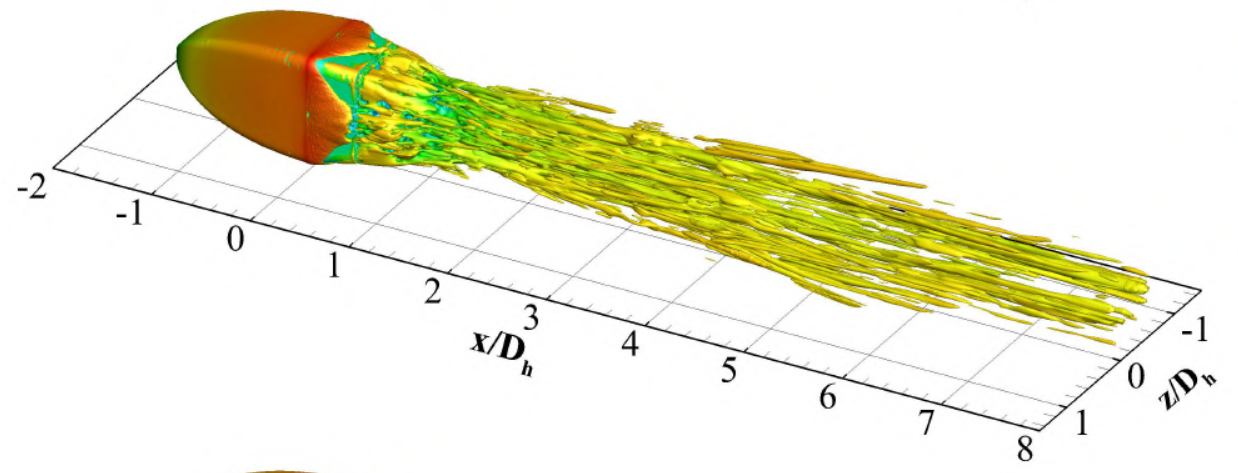

$\mathrm{U}_{\mathrm{x}} / \mathrm{U}_{\text {ref }}$

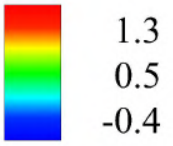

c)

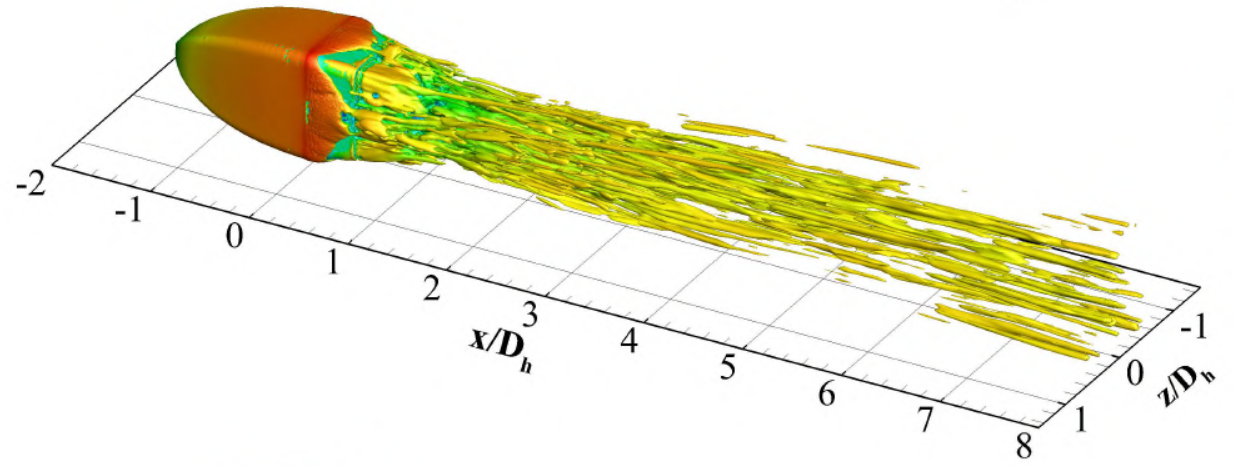

$\mathrm{U}_{\mathrm{x}} / \mathrm{U}_{\text {ref }}$

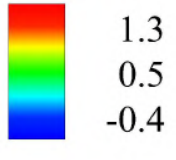

d)

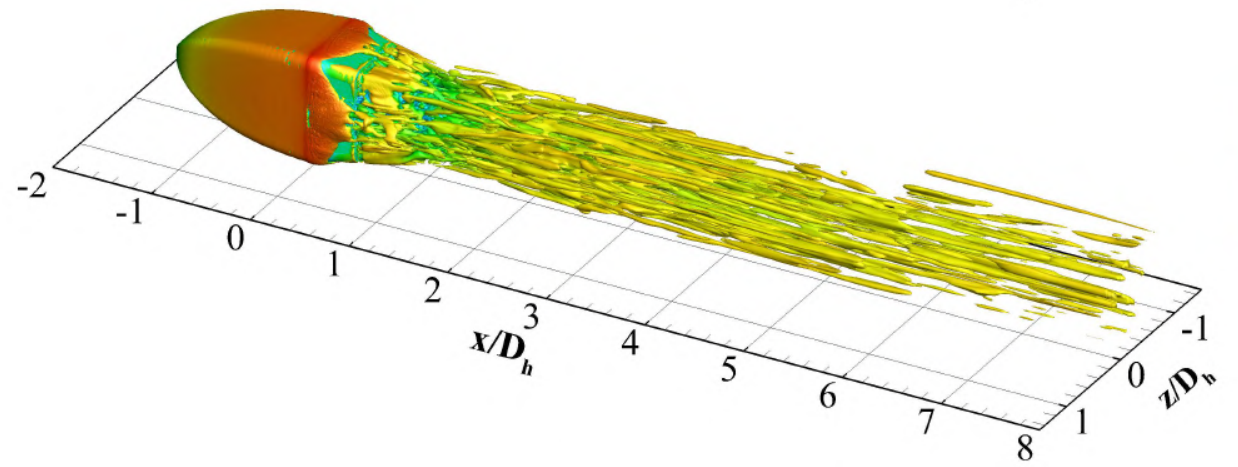

$\mathrm{U}_{\mathrm{x}} / \mathrm{U}_{\text {ref }}$

Figure C.15: $\mathrm{Q} /\left(\mathrm{U}_{\text {ref }} / \mathrm{D}_{\mathrm{h}}\right)^{2}=2.4 \times 10^{-1}$ isosurfaces calculated based on sequentially time-averaged flow fields with an averaging period of $25 \mathrm{~K}$ $\mathbf{H}$ cycles in the straight-tail configuration in the absence of small-scale turbulence 
a)

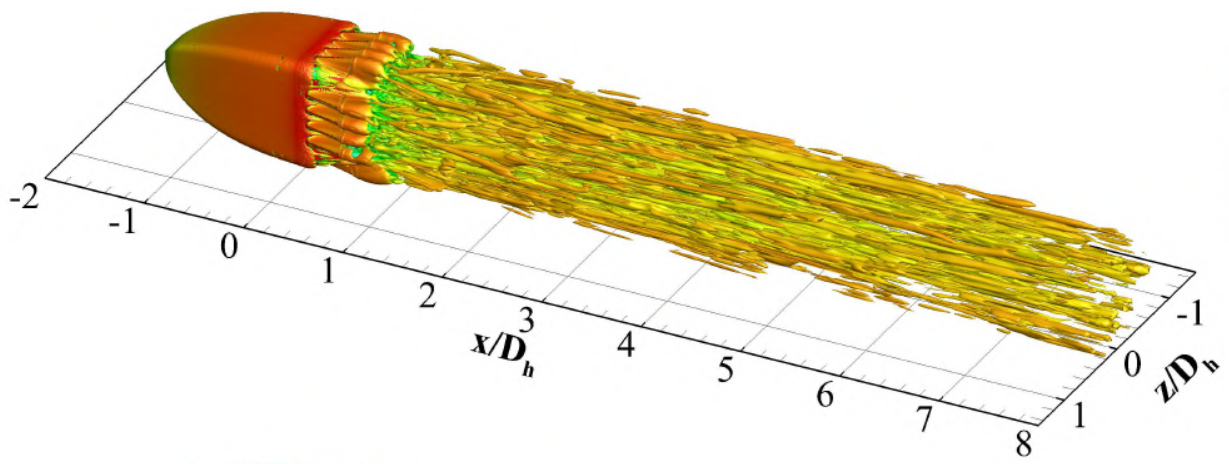

b)

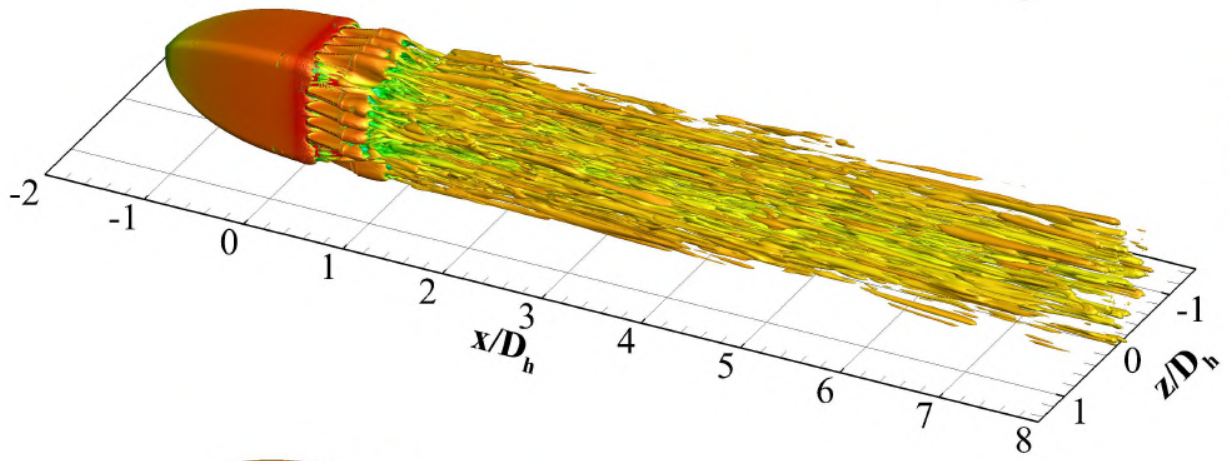

c)

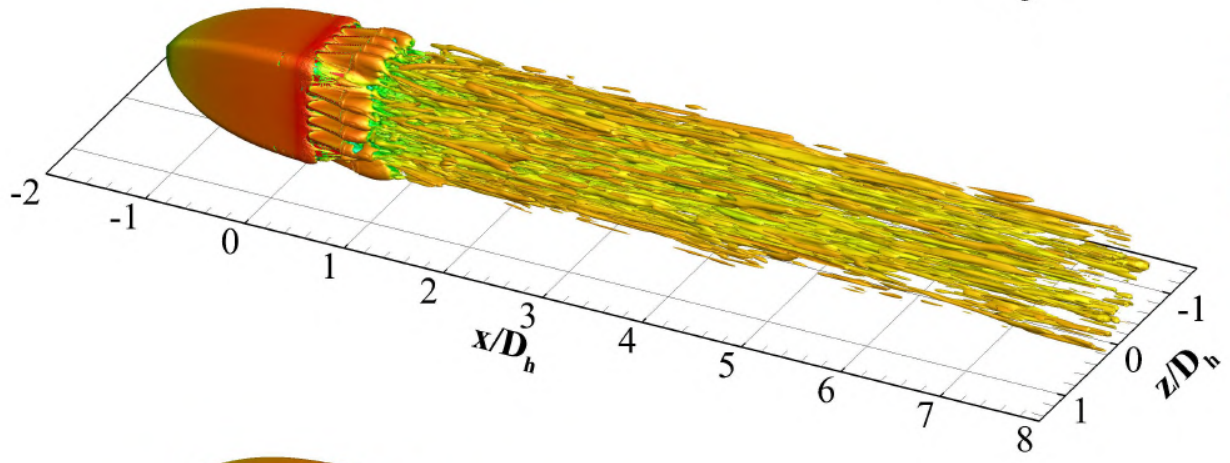

d)

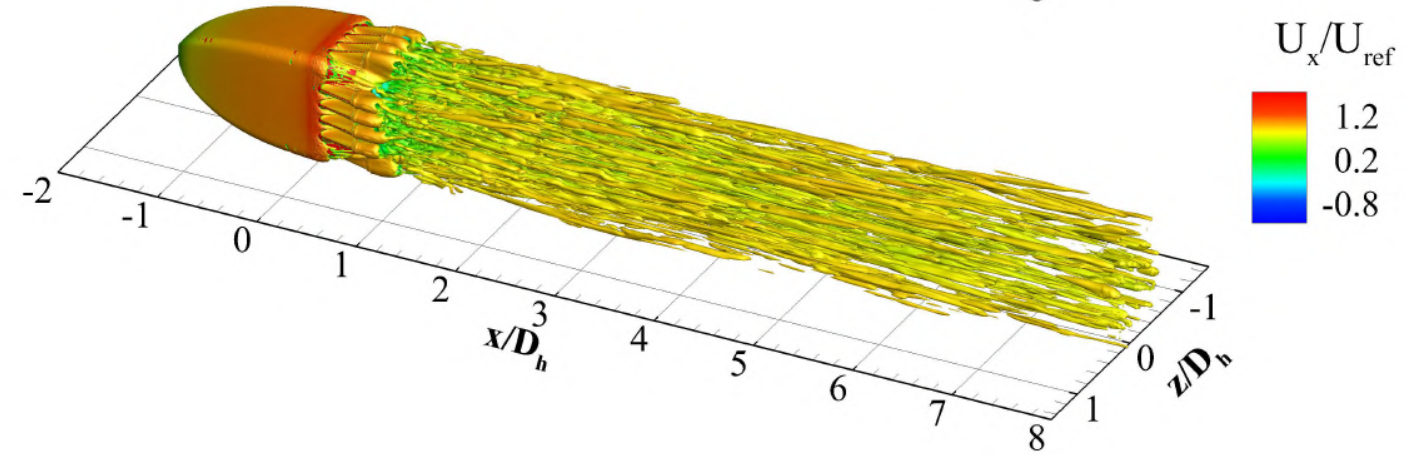

Figure C.16: $\mathrm{Q} /\left(\mathrm{U}_{\mathrm{ref}} / \mathrm{D}_{\mathrm{h}}\right)^{2}=2.4 \times 10^{-1}$ isosurfaces calculated based on sequentially time-averaged flow fields with an averaging period of $25 \mathrm{~K}-\mathrm{H}$ cycles in the lobed-tail configuration in the absence of small-scale turbulence 
a)

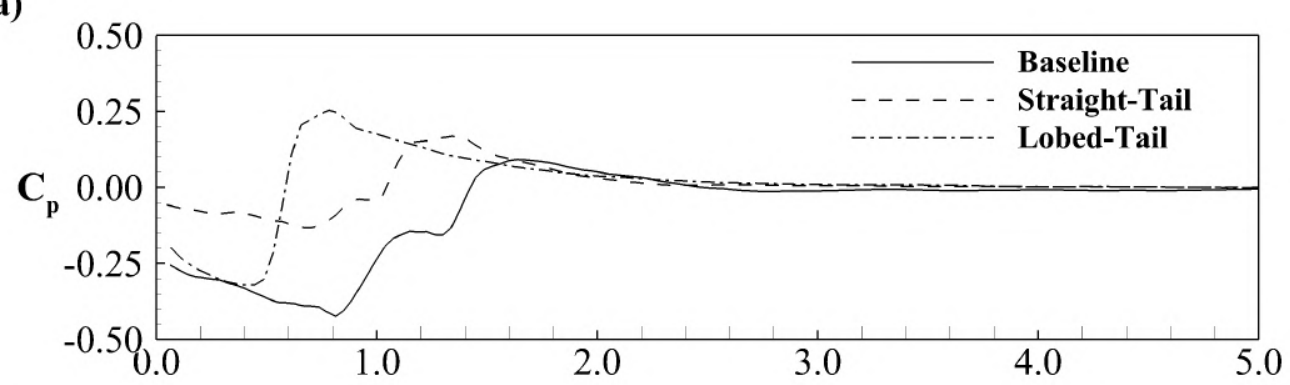

b)

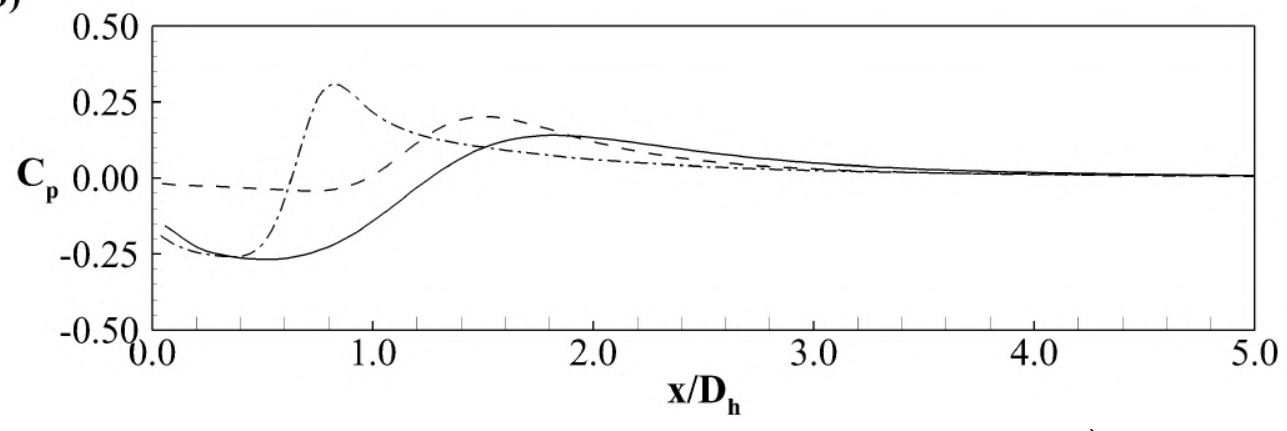

Figure C.17: Pressure variation along the wake centerline a) in the absence of small-scale turbulence, b) in the presence of small-scale turbulence

a)

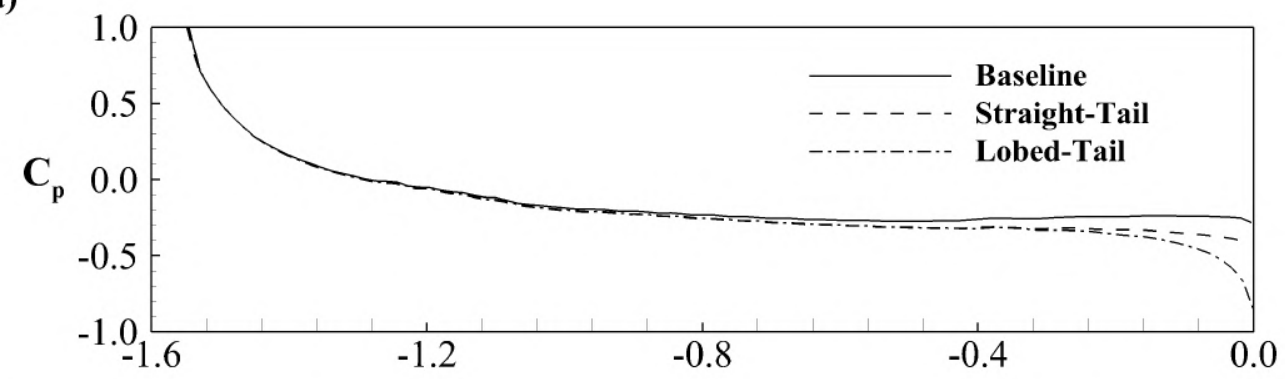

b)

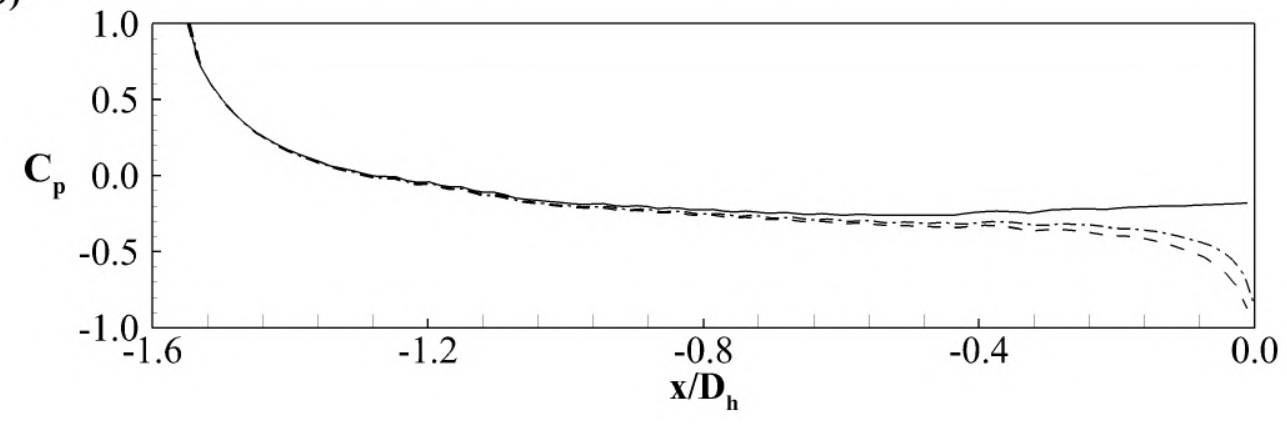

Figure C.18: Pressure variation along the bluff-body surface in the $x-z$ plane a) in the absence of small-scale turbulence, b) in the presence of small-scale turbulence 


\section{Appendix D}

\section{Influence of Compressibility on Lobed-Mixer Performance}

The development of the free-shear layer in close vicinity of the lobed-mixer discharge plane is influenced by compressibility, obliqueness of the initial perturbation in the free-shear layer with respect to the streamwise direction, and density and velocity ratios of the two streams. The effects of compressibility in the mixing dynamics of coflowing streams have conventionally been represented by the convective Mach number, $M_{c}$, which is defined with respect to each of the two streams as follows [153-158]:

$$
M_{c_{1}}=\frac{U_{1}-U_{c}}{a_{1}}, M_{c_{2}}=\frac{U_{c}-U_{2}}{a_{2}}
$$

where $U$ is the freestream velocity, $U_{c}$ is the convective velocity of the large-scale structures within the free-shear layer, $a$ is the speed of sound, and the subscripts 1 and 2 denote the high-speed and low-speed streams, respectively. $M_{c_{1}}$ and $M_{c_{2}}$ can be related by:

$$
M_{c_{1}}=\left(\frac{\gamma_{1}}{\gamma_{2}}\right)^{1 / 2} M_{c_{2}}
$$

where $\gamma$ is the specific heat ratio. For two streams with equal specific-heat ratios and equal densities, the convective velocity and the two convective Mach numbers are reduced to:

$$
\begin{gathered}
U_{c}=\frac{U_{1} a_{2}+U_{2} a_{1}}{a_{1}+a_{2}} \\
M_{c_{1}}=M_{c_{2}}=M_{c}=\frac{\Delta U}{a_{1}+a_{2}}
\end{gathered}
$$

where $\Delta U$ is the velocity difference, $U_{1}-U_{2}[154]$. In the case where the initial perturbations leading to the development of the convective flow structures in the free-shear layer are oblique, the effective value of $M_{c}$ to describe the effects of compressibility is determined as [159-163]:

$$
M_{c_{e f f}}=M_{c} \cos \beta
$$


It is evident from this expression that compressibility effects are attenuated with increased obliqueness of the perturbation. Measurements of Papamoschou [159] suggest that when the two convective Mach numbers are not of the same magnitude, the higher-speed stream influences the dynamics of the free-shear layer to a greater extent owing to the larger role of compressibility in this stream. With increasing values of $M_{c}$, the disturbances in the shear layer have been observed to amplify at a lower rate under the influence of the $\mathrm{K}-\mathrm{H}$ instability mode, resulting in delayed roll-up of the shear layer into the normal vortices [164]. At the same time, the dominant frequency of the K-H instability, expressed in non-dimensional form as a Strouhal number defined as:

$$
S t_{\theta}=\frac{f \theta}{U_{c}}
$$

has been observed to increase with increasing values of $M_{c}$, where $f$ is the shedding frequency and $\theta$ is the shear-layer momentum thickness just downstream of the trailing edge [165]. The Strouhal number of this instability for incompressible conditions has been observed to be about 0.016. [166]. The flow physics are observed to be affected by compressibility even before the convective Mach numbers reach the supersonic regime. Through direct numerical simulations, Karimi [167] observed that the normal vortices were deformed or even suppressed by the effect of compressibility while the formation of the streamwise vortices remained largely unaffected. In the absence of normal vortices, it was seen that the streamwise vortices linked with each other, forming long streamwise streaks. These developments induced by compressibility result in a degradation in mixing of the two streams since the primary instability mechanism yielding mass and momentum transfer is partly or fully stabilized.

When the compressibility effects are strong, large gradients of density across the mixing layer are observed. Measurements of Brown and Roshko [164] have shown that deviation from unity of the static density ratio between the two streams prompted by $M_{c}$ values in the compressible range has relatively small effects on the dynamics of the free-shear layer.

The growth of the mixing layer in streamwise direction is mainly attributed to the successive amalgamation of the normal vortices [120]. The growth rate of the mixing 
layer is observed to be proportional to the velocity difference between the two coflowing streams, thus inversely proportional to their velocity ratio, $U_{2} / U_{1}[156,168,169]$. Similarly, the streamwise growth rate of perturbations under the Kelvin-Helmholtz instability mode increases with smaller velocity ratios resulting in increased diameter of the the normal vortices while the magnitude of the normal vorticity is proportional to the velocity difference between the two coflowing fluids. The magnitude of the streamwise vorticity becomes higher at smaller velocity ratios due to the increased velocity difference between the radially oriented secondary flows prevailing along the sidewalls of the the lobes, promoting stronger induced velocities by streamwise vortices. This suggests that the pinch-off of the weaker normal vortices due to interaction with the stronger streamwise vortices appears to occur earlier with smaller velocity ratios. 
Appendix E

Technical Drawings of the Bluff-Body Model and Tails 
D Boat-Tail Extension

C

B

A

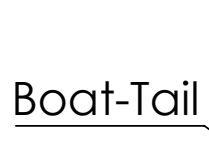

$\circ$
D

Bluff-Body Model

○

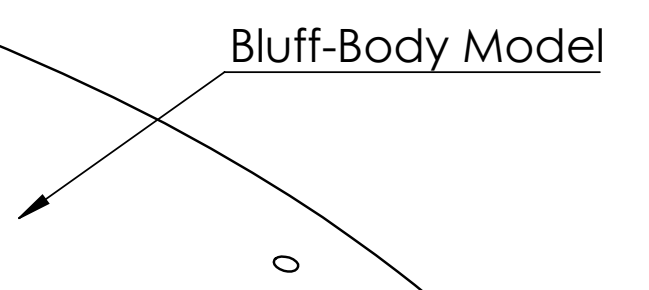

C

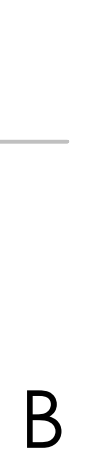

Bluff-Body Model \& Boat-Tail Assembly

\begin{tabular}{|c|c|c|}
\hline \multicolumn{2}{|c|}{$\begin{array}{l}\text { DESIGAEE BY: } \\
\text { DARE: Caylan } \\
\text { Utk17-07-28 }\end{array}$} & Bluff-Body Model \& Boat-Tail Assembly \\
\hline SIE & (1) 5 & CARLETON UNIVERSITY \\
\hline
\end{tabular}

TOLERANCES

+/- $0.1 \mathrm{~mm}$ unless otherwise

DRAWING NUMBER SCALE

$1: 2.2$ DIMENSIONS

MATERIAL

ABS

Tha drawing is our property; it cannot be reproduced or communicated without our written agreement. 
D

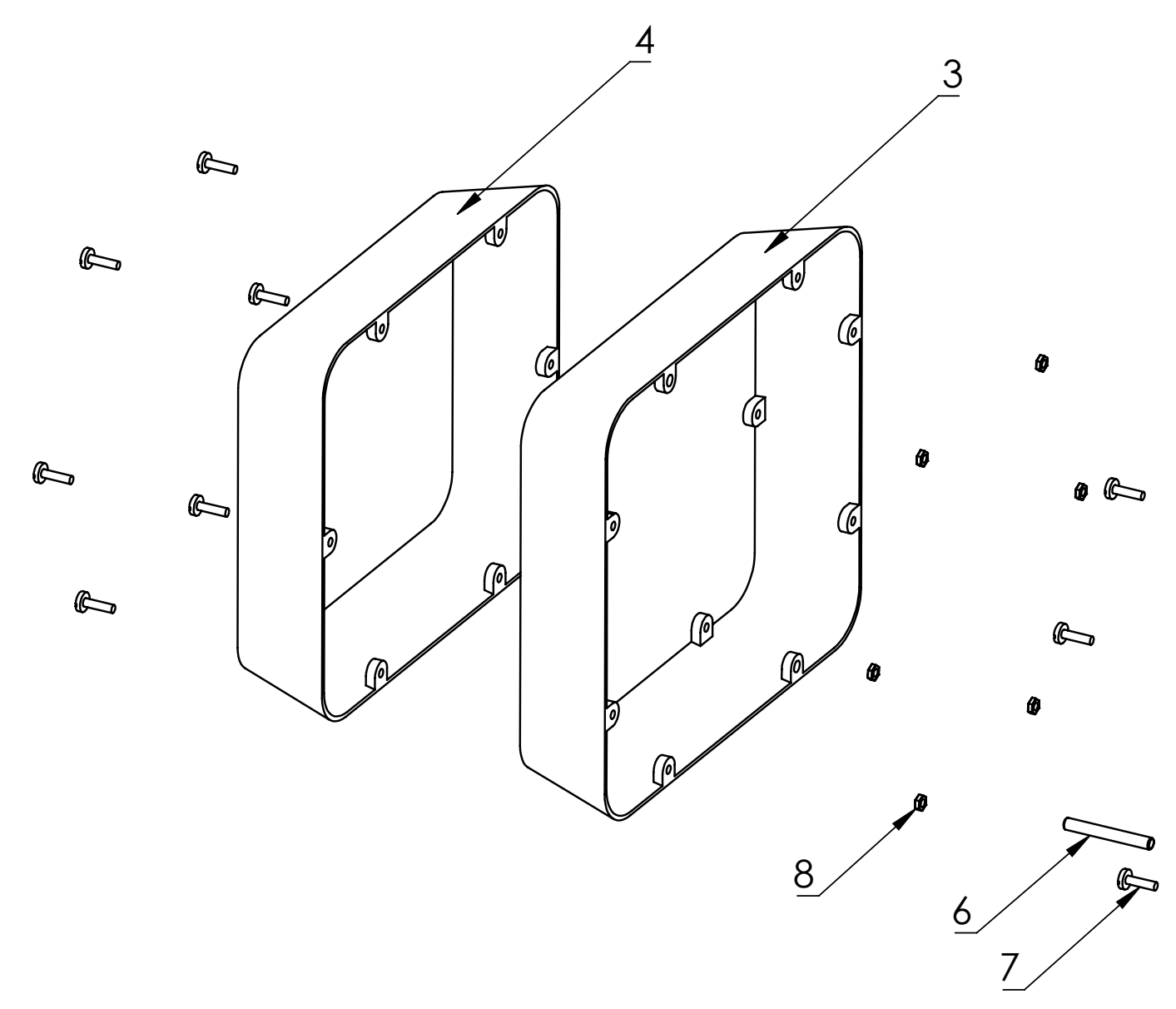

B

\begin{tabular}{|c|c|c|}
\hline Part \# & Name & Qty \\
\hline 1 & Bluff-Body Model & 1 \\
\hline 2 & Back Plate & 1 \\
\hline 3 & Boat-Tail & 1 \\
\hline 4 & Straight Boat-Tail Extension & 1 \\
\hline 5 & Helicoil Insert with \#6-32 Internal Thread & 3 \\
\hline 6 & Dowel Pin (3/16 $1-1 / 2$ in) & 2 \\
\hline 7 & \#6-32 UNF Machine Screw & 12 \\
\hline 8 & \#6-32 UNF Machine Screw Nut & 12 \\
\hline
\end{tabular}

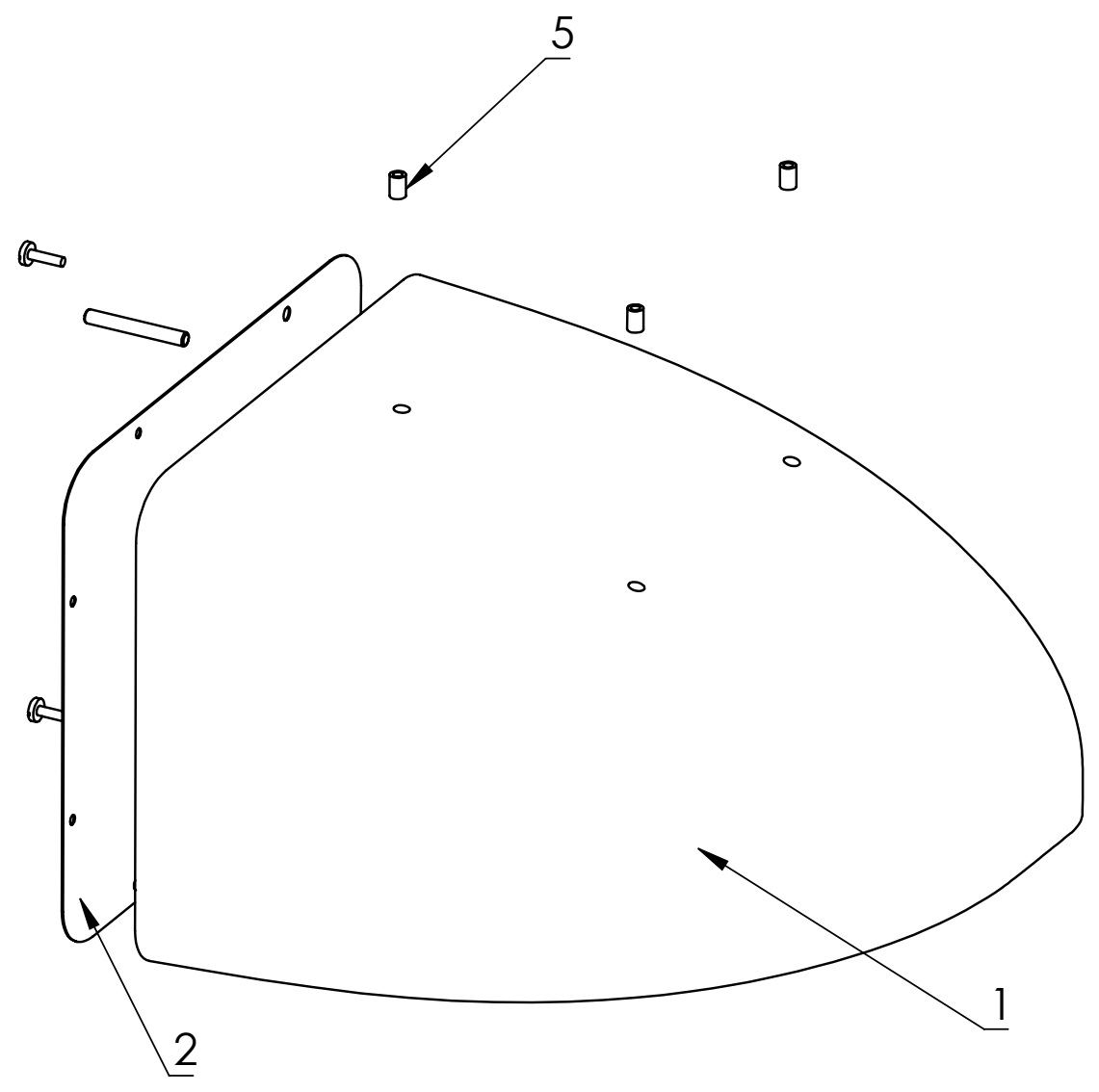

\begin{tabular}{|c|c|c|c|c|c|c|}
\hline \multicolumn{2}{|c|}{$\begin{array}{l}\text { DESIGNED BY: } \\
\text { Utku Caylan } \\
\text { DATE: } \\
\mathbf{2 0 1 7 - 0 7 - 2 8}\end{array}$} & \multicolumn{5}{|c|}{$\begin{array}{c}\text { Bluff-Body Model \& Boat-Tail Assembly: } \\
\text { Exploded View }\end{array}$} \\
\hline A4 4 & (Q) $\square$ & \multicolumn{5}{|c|}{ CARLETON UNIVERSITY } \\
\hline \multicolumn{2}{|l|}{ TOLERANCES } & $\begin{array}{c}\text { DRAWING NUMBER } \\
1\end{array}$ & $\begin{array}{l}\text { SCALE } \\
1: 3.2\end{array}$ & $\begin{array}{l}\text { DIMENSIONS } \\
\mathbf{m m}\end{array}$ & $\begin{array}{l}\text { MATERIAL } \\
\text { ABS }\end{array}$ & $\begin{array}{l}\text { SHEET } \\
\mathbf{2} / \mathbf{2}\end{array}$ \\
\hline
\end{tabular}

3 


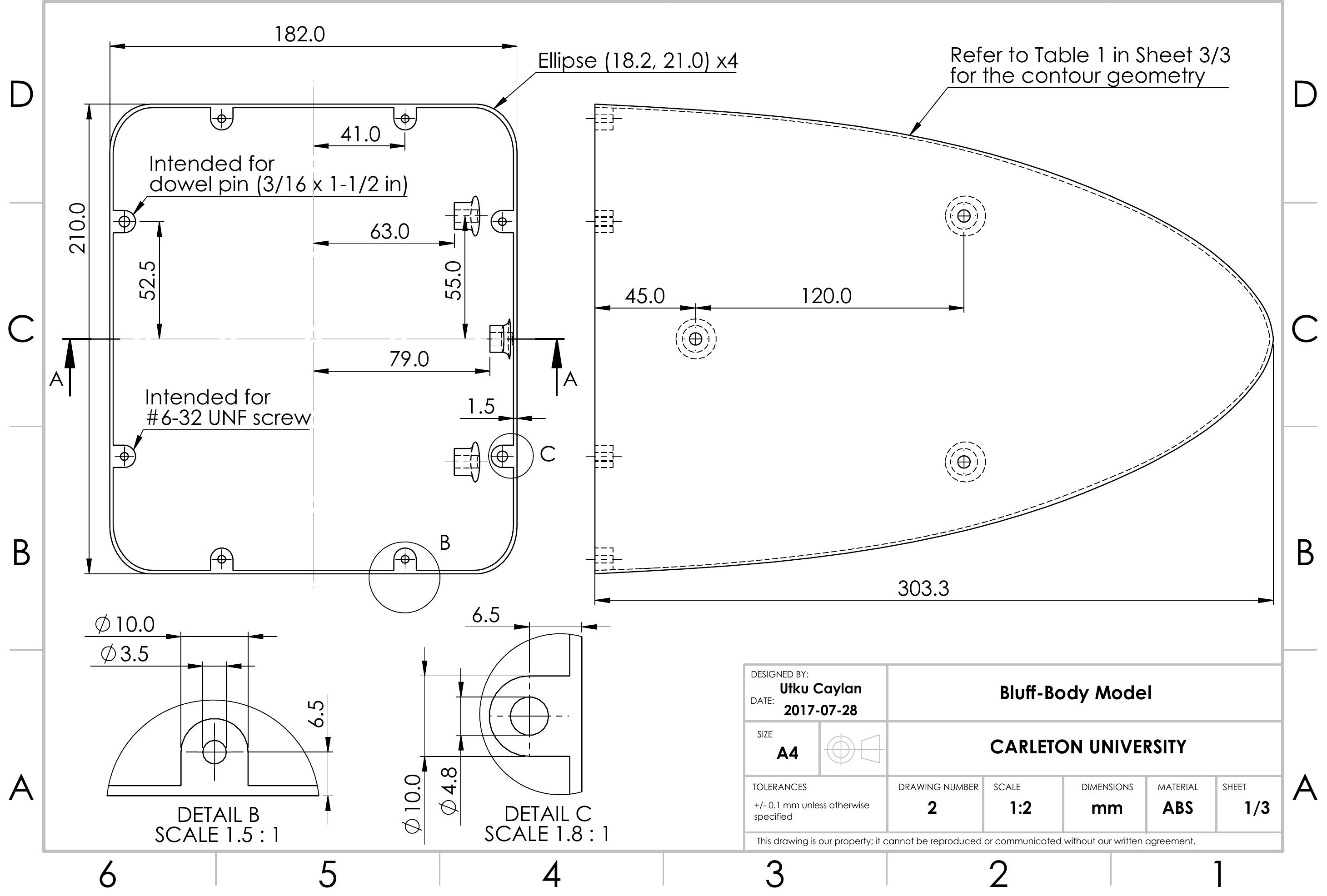


D
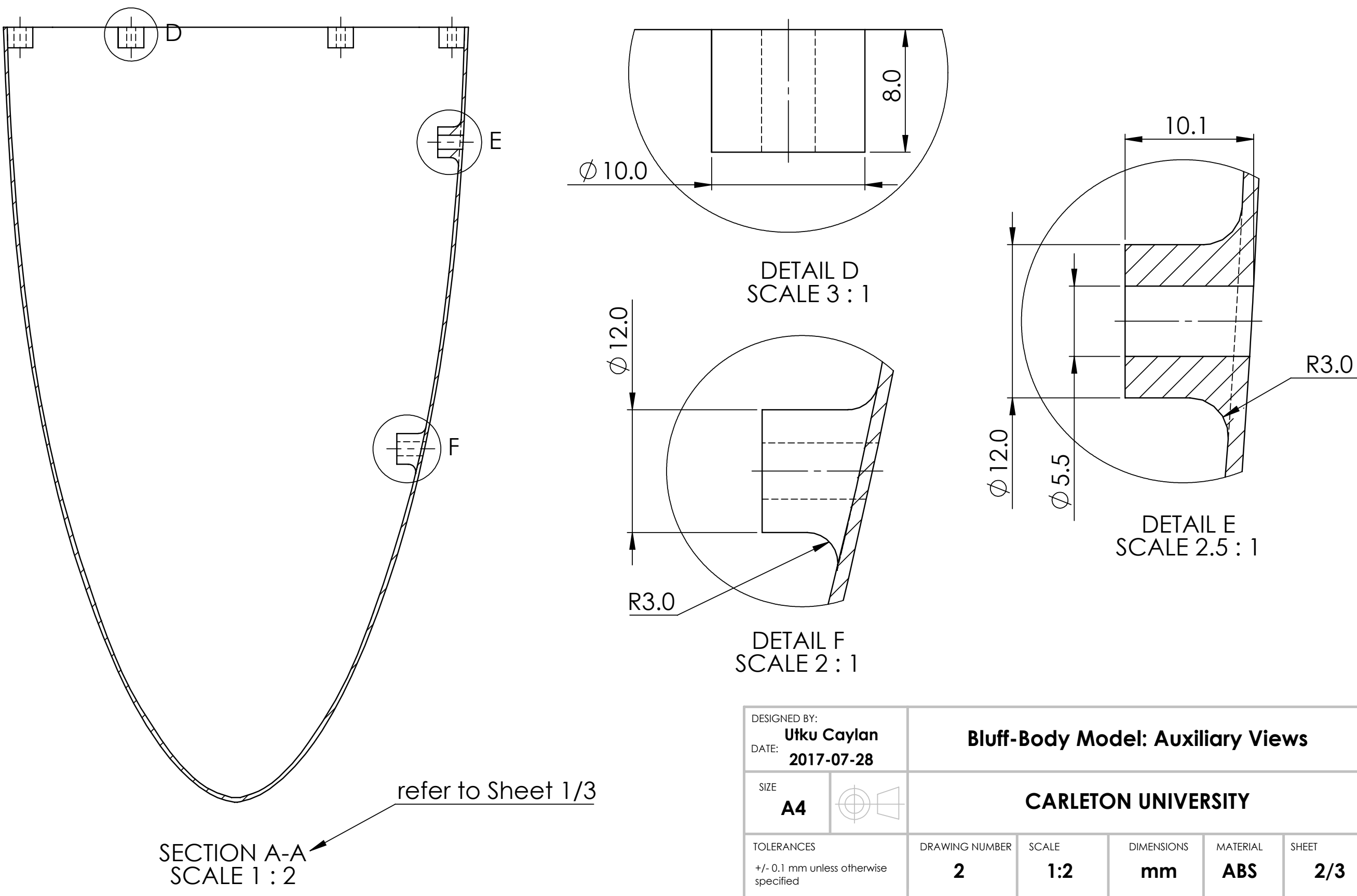

B

A SCALE $1: 2$

\begin{tabular}{|c|c|c|c|c|c|c|}
\hline \multicolumn{2}{|c|}{$\begin{array}{l}\text { DESIGNED BY: } \\
\text { Utku Caylan } \\
\text { DAAE: } \\
2017-07-28\end{array}$} & \multirow{2}{*}{\multicolumn{5}{|c|}{$\begin{array}{c}\text { Bluff-Body Model: Auxiliary Views } \\
\text { CARLETON UNIVERSITY }\end{array}$}} \\
\hline $\begin{array}{l}\text { SIE } \\
\text { A4 }\end{array}$ & (1) $\square$ & & & & & \\
\hline \multicolumn{2}{|c|}{ 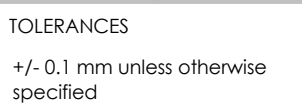 } & $\begin{array}{l}\text { DRAWING NUMBER } \\
\mathbf{2}\end{array}$ & $\begin{array}{l}\text { SCALE } \\
1: 2\end{array}$ & $\begin{array}{c}\text { DIMENSIONS } \\
\text { mm }\end{array}$ & $\begin{array}{c}\text { MATERAL } \\
\text { ABS }\end{array}$ & $\begin{array}{l}\text { SHEET } \\
2 / 3\end{array}$ \\
\hline \multicolumn{7}{|c|}{ Urw witten agreemer } \\
\hline \multicolumn{2}{|l|}{3} & & 2 & & \\
\hline
\end{tabular}


Table 1

\begin{tabular}{|c|c|c|c|c|c|}
\hline Wireframe No & $x / L$ & $S / L$ & $T / L$ & $a / L$ & $b / L$ \\
\hline 1 & 0.00000 & 0.55391 & 0.48005 & 0.06924 & 0.06001 \\
\hline 2 & 0.32971 & 0.51434 & 0.44543 & 0.06429 & 0.05569 \\
\hline 3 & 0.57369 & 0.44049 & 0.37877 & 0.05506 & 0.04735 \\
\hline 4 & 0.67590 & 0.39037 & 0.33551 & 0.04880 & 0.04194 \\
\hline 5 & 0.75173 & 0.34289 & 0.29489 & 0.04286 & 0.03686 \\
\hline 6 & 0.85724 & 0.25981 & 0.22341 & 0.03248 & 0.02793 \\
\hline 7 & 0.90010 & 0.21576 & 0.18675 & 0.02697 & 0.02334 \\
\hline 8 & 0.97263 & 0.10551 & 0.09074 & 0.01319 & 0.01134 \\
\hline 9 & 0.99242 & 0.05275 & 0.04379 & 0.00659 & 0.00547 \\
\hline 10 & 0.99736 & 0.02638 & 0.02189 & 0.00330 & 0.00274 \\
\hline 11 & 0.99901 & 0.01319 & 0.01095 & 0.00165 & 0.00138 \\
\hline 12 & 0.99984 & 0.00659 & 0.00554 & 0.00082 & 0.00069 \\
\hline
\end{tabular}

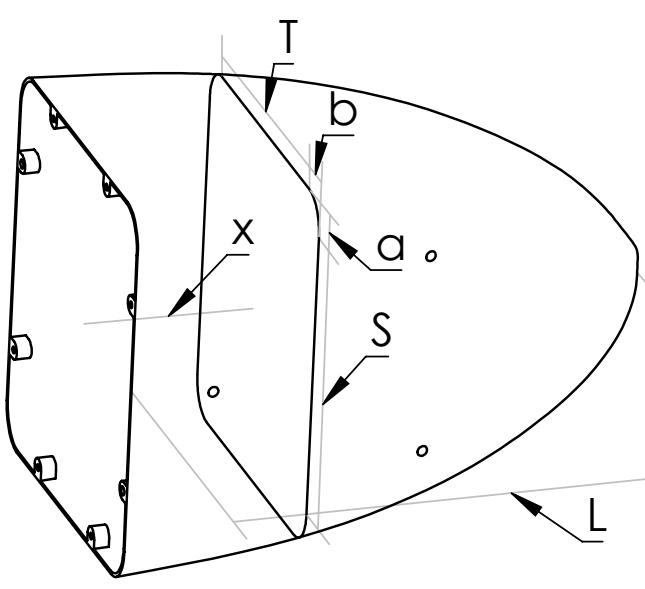

Note 2:

Lofting of the given wireframes in making of the bluff-body surface is performed using the non-uniform rational basis spline (NURBS) mathematical model. The shape of the model surface is determined by control points which lie on the same diagonal plane.

\section{Note 1:}

$a$ is fillet major-axis length of a given wireframe.

$b$ is fillet minor-axis length of a given wireframe.

$L$ is model length.

$x$ is axial location of a given wireframe.

$S$ is straight-segment length along the sides of a given wireframe.

$\mathrm{T}$ is straight-segment length at the top/bottom of a given wireframe.

\begin{tabular}{|c|c|c|c|c|c|c|}
\hline \multicolumn{2}{|c|}{$\begin{array}{l}\text { DESIGNED BY: } \\
\text { Utku Caylan } \\
\text { DATE: } \\
\mathbf{2 0 1 7 - 0 7 - 2 8}\end{array}$} & \multirow{2}{*}{\multicolumn{5}{|c|}{$\begin{array}{c}\text { Bluff-Body Model: Contour Geometry } \\
\text { CARLETON UNIVERSITY }\end{array}$}} \\
\hline${ }^{\text {SIIE }}$ A4 & (1) [ & & & & & \\
\hline \multicolumn{2}{|c|}{$\begin{array}{l}\text { TOLERANCES } \\
+1-0.1 \mathrm{~mm} \text { unless otherwise } \\
\text { specified }\end{array}$} & $\begin{array}{l}\text { DRAWING NUMBER } \\
\mathbf{2}\end{array}$ & $\begin{array}{l}\text { SCALE } \\
1: 5\end{array}$ & $\begin{array}{c}\text { DIMENSIONS } \\
\mathbf{m m}\end{array}$ & $\begin{array}{l}\text { MATERIAL } \\
\text { ABS }\end{array}$ & $\begin{array}{l}\text { SHEET } \\
\mathbf{3} / \mathbf{3}\end{array}$ \\
\hline \multicolumn{7}{|c|}{ eement. } \\
\hline
\end{tabular}




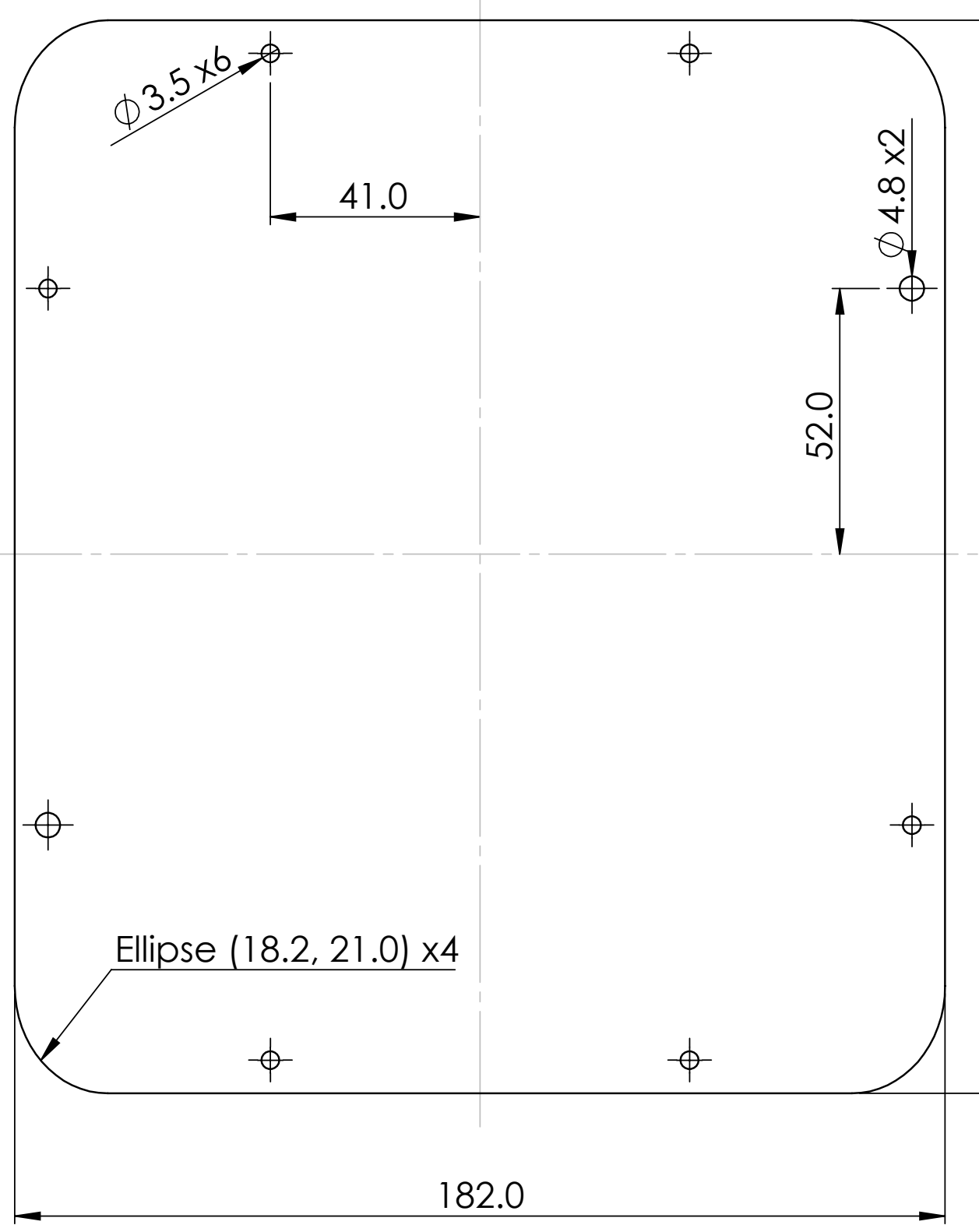

A

C
B 2017-07-28

\section{Back Plate}

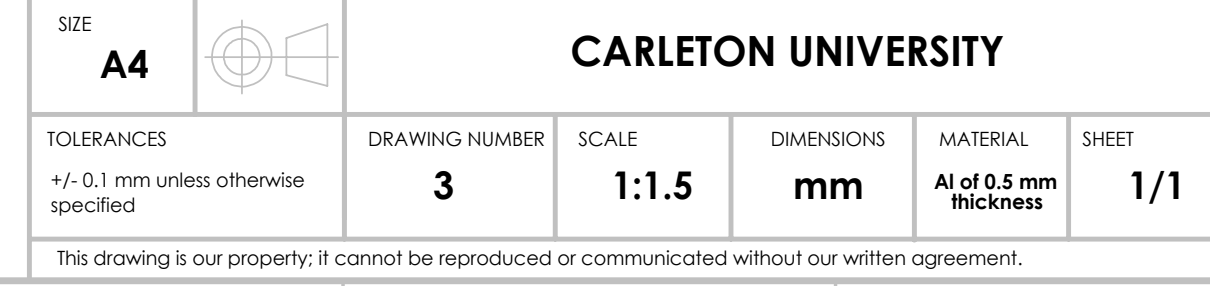

C

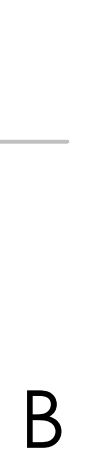

3

2 


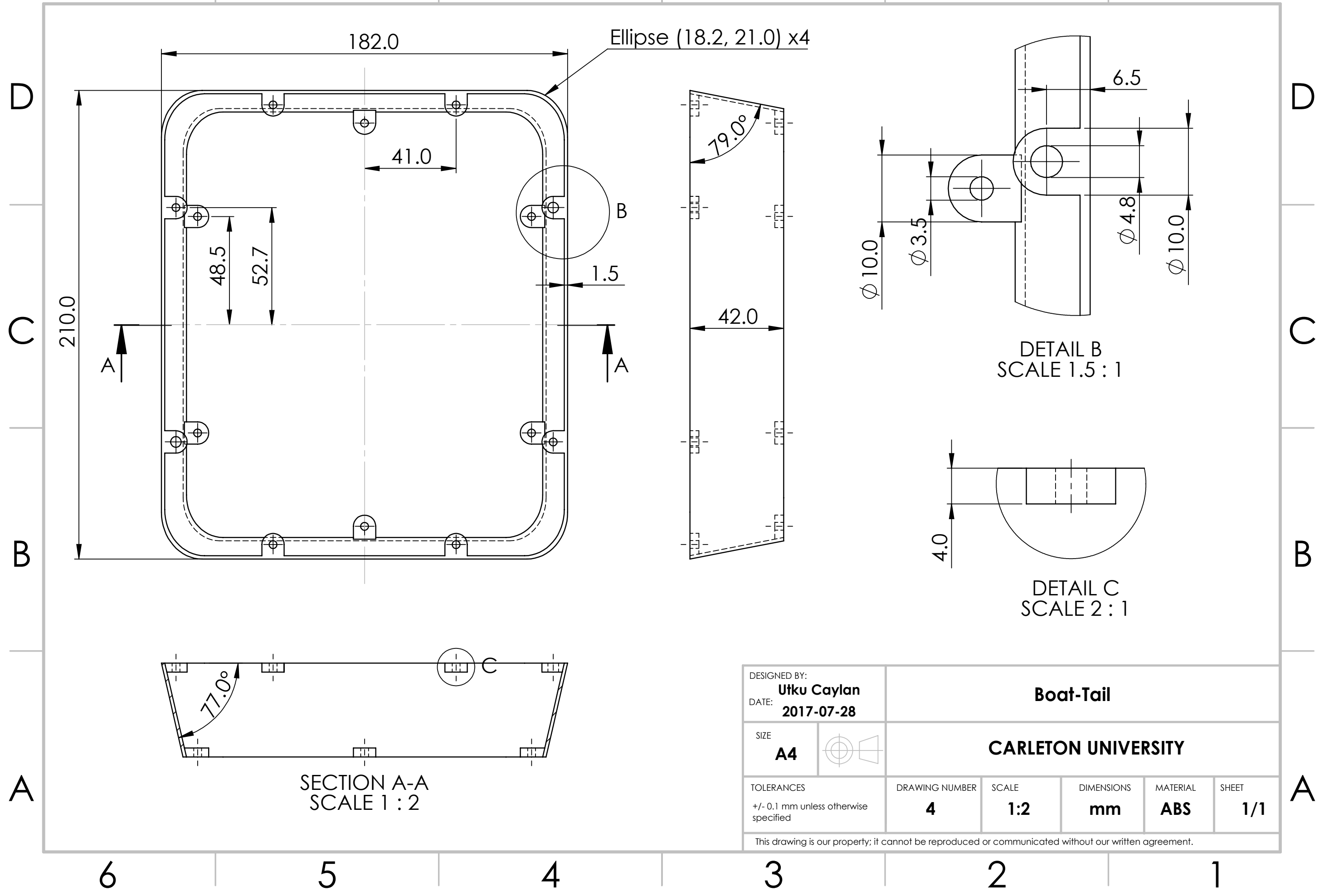


D
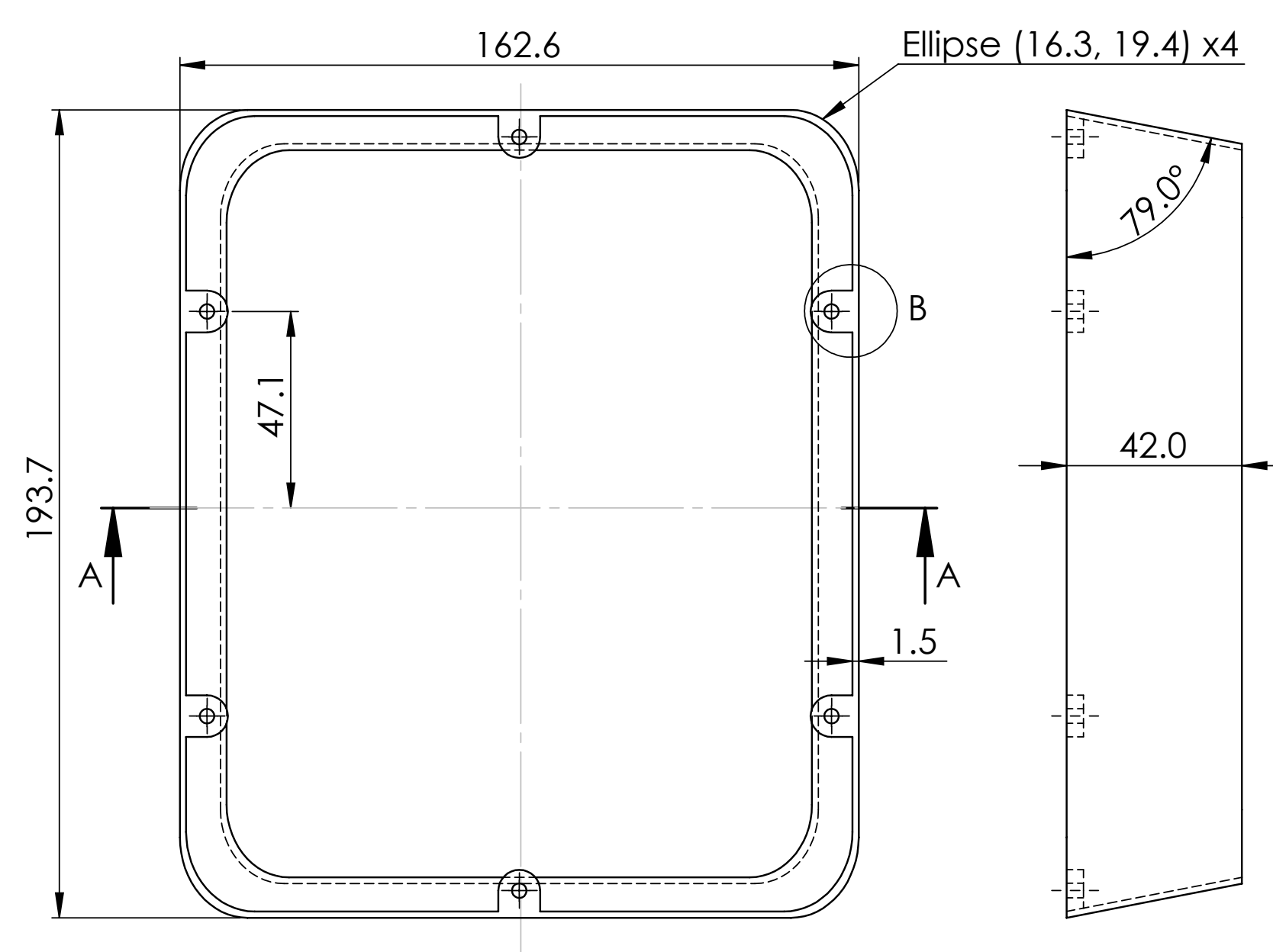

C
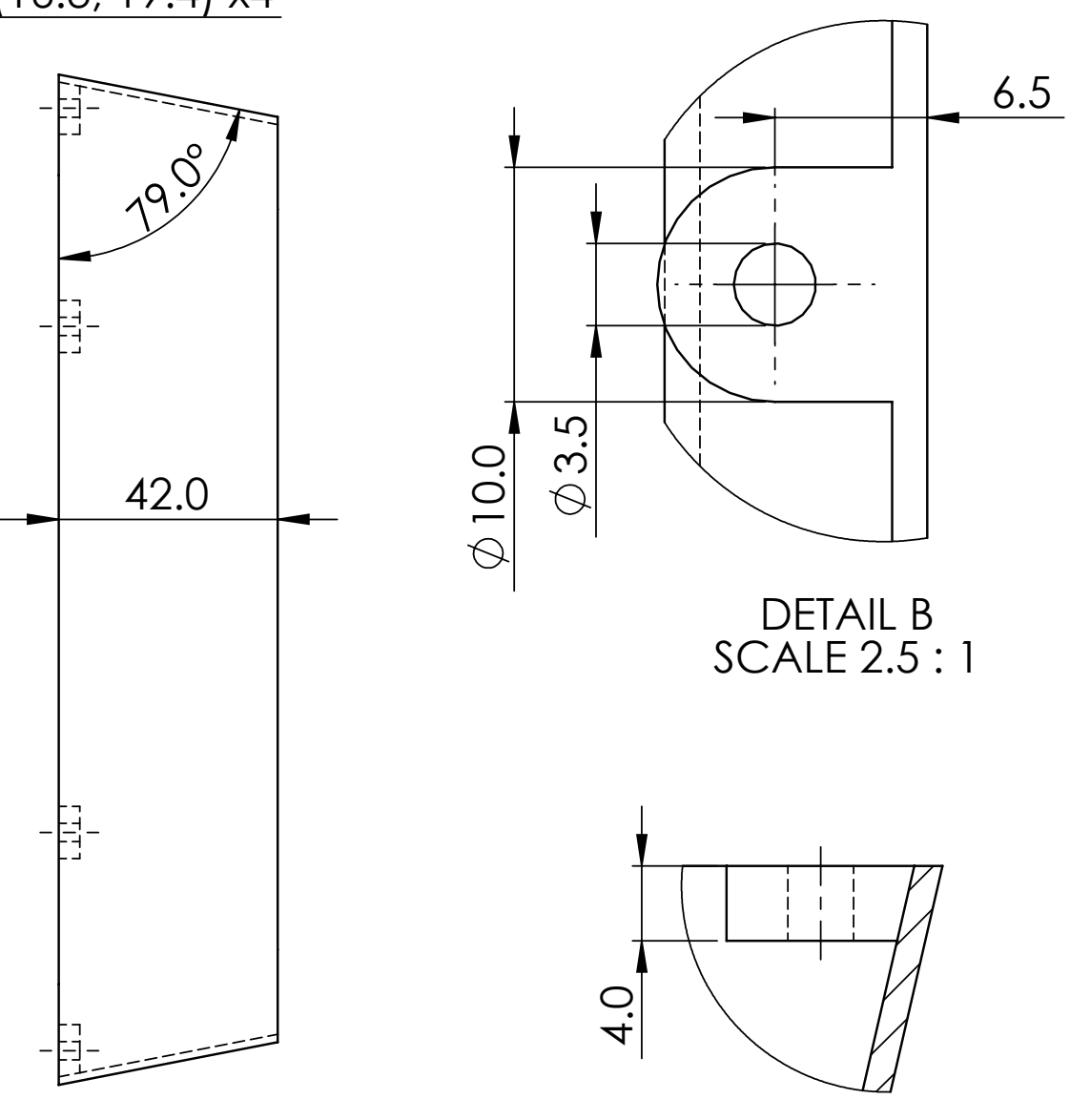

D SCALE $2.5: 1$
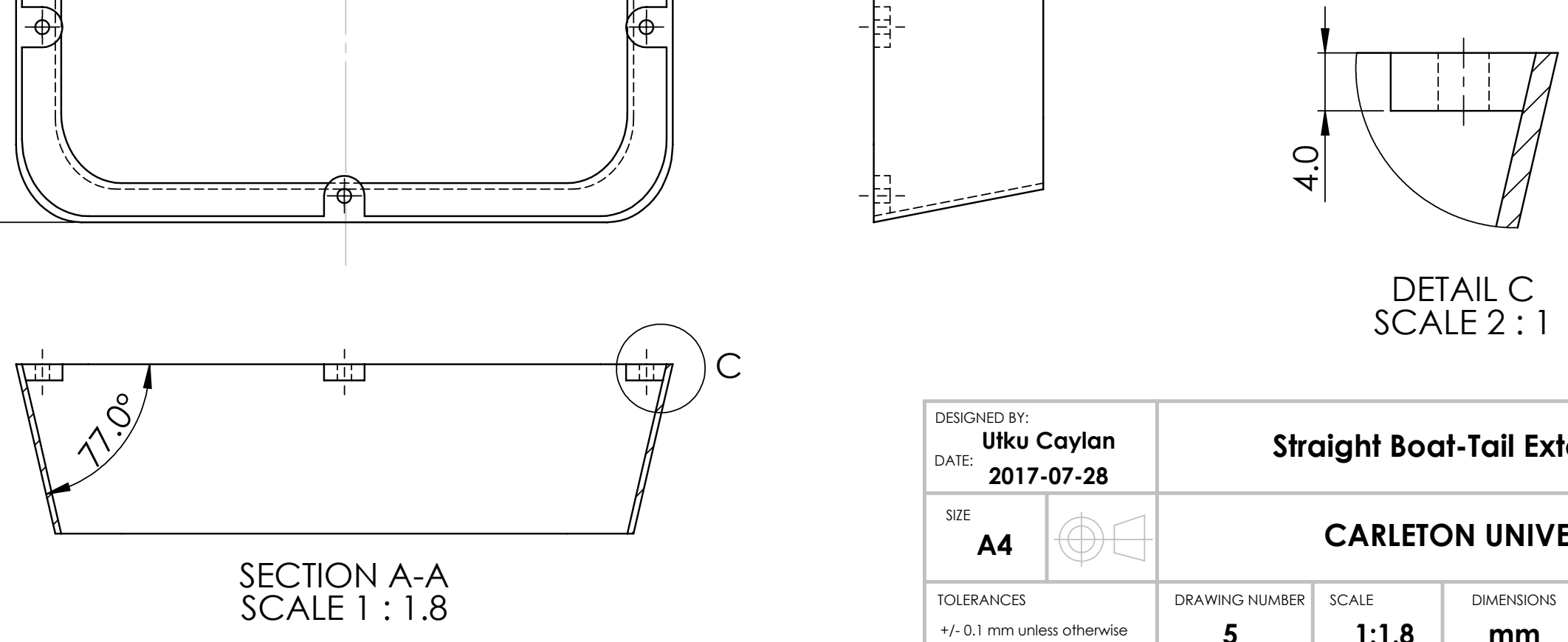

DETAIL C
SCALE 2:

B

C

A

SECTION A-A

\begin{tabular}{|c|c|c|c|c|}
\hline $\begin{array}{c}\text { DESIGNED BY: } \\
\text { UtkU Caylan } \\
\text { DAAEE }\end{array}$ & \multicolumn{4}{|c|}{ Straight Boat-Tail EXtension } \\
$2017-07-28$
\end{tabular}



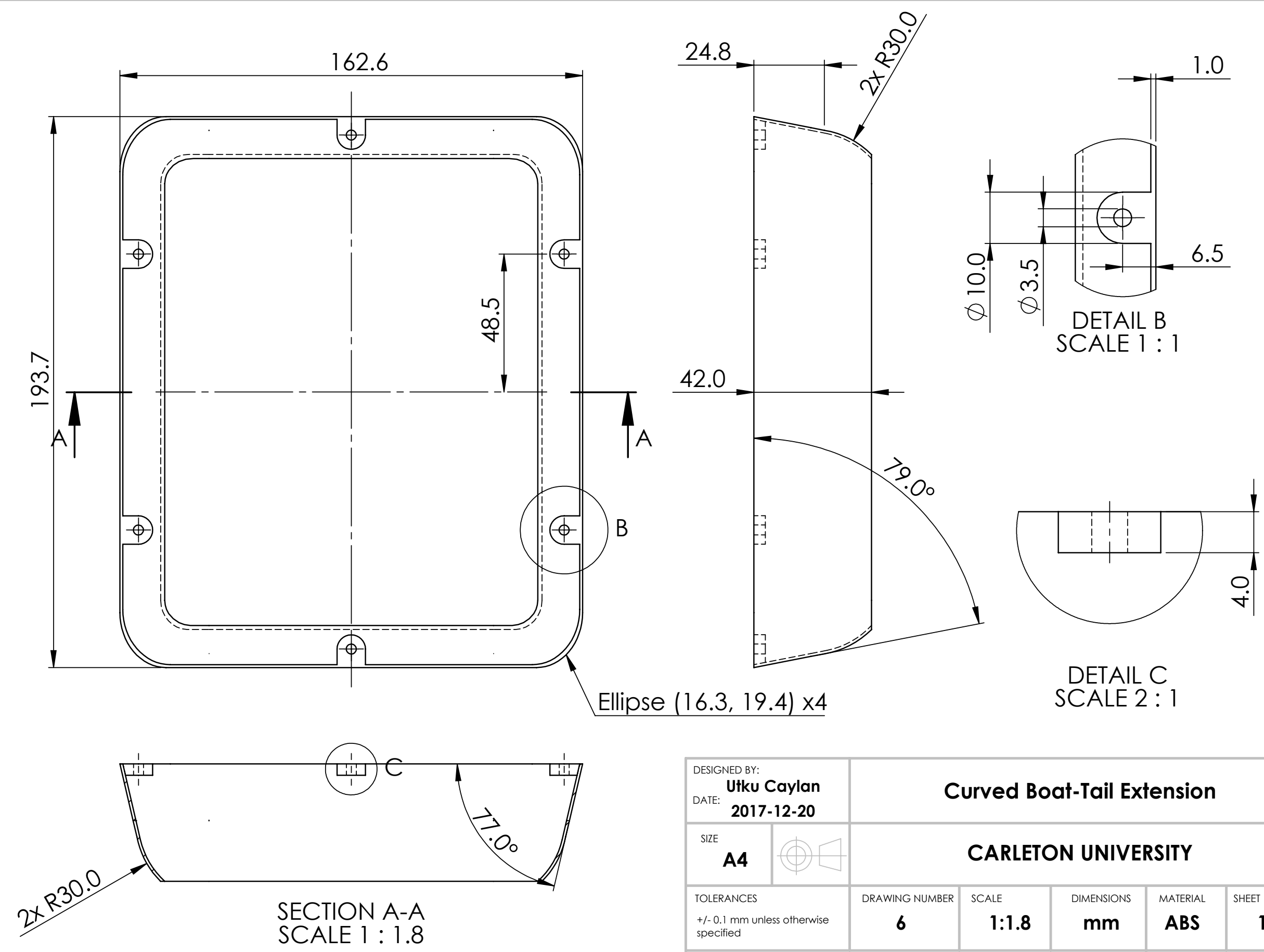

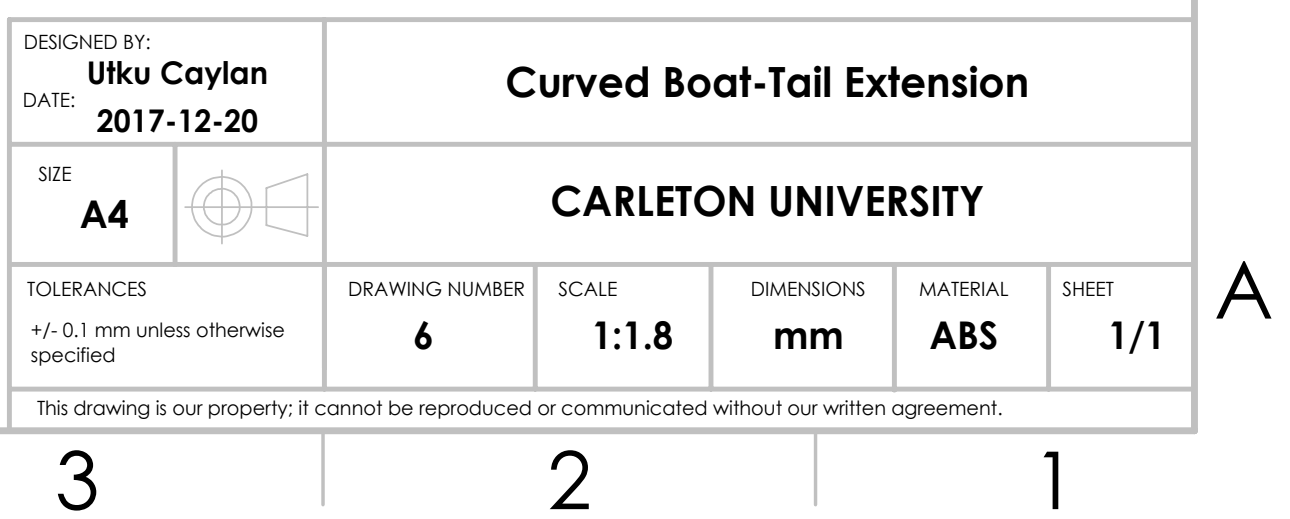


D

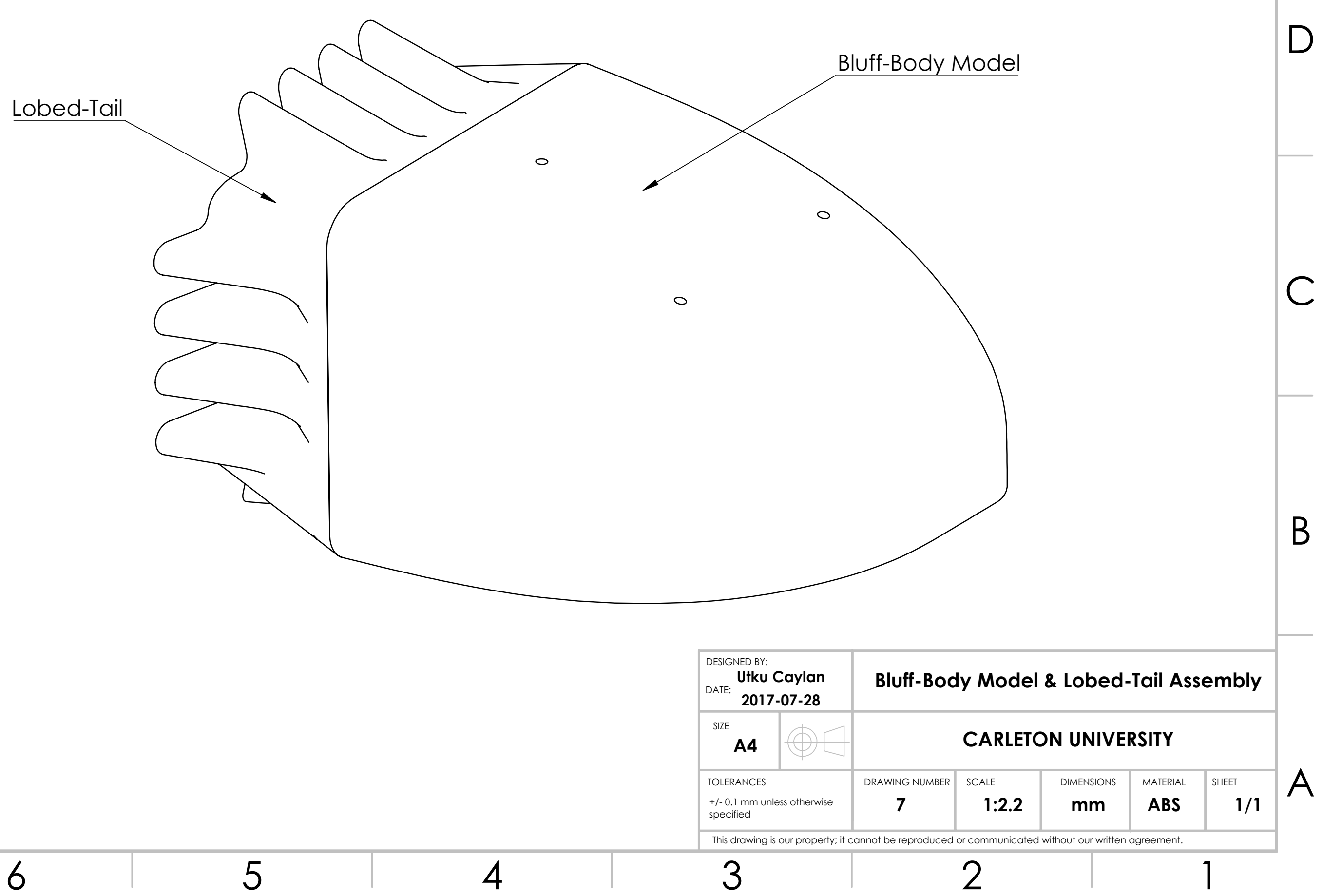


D

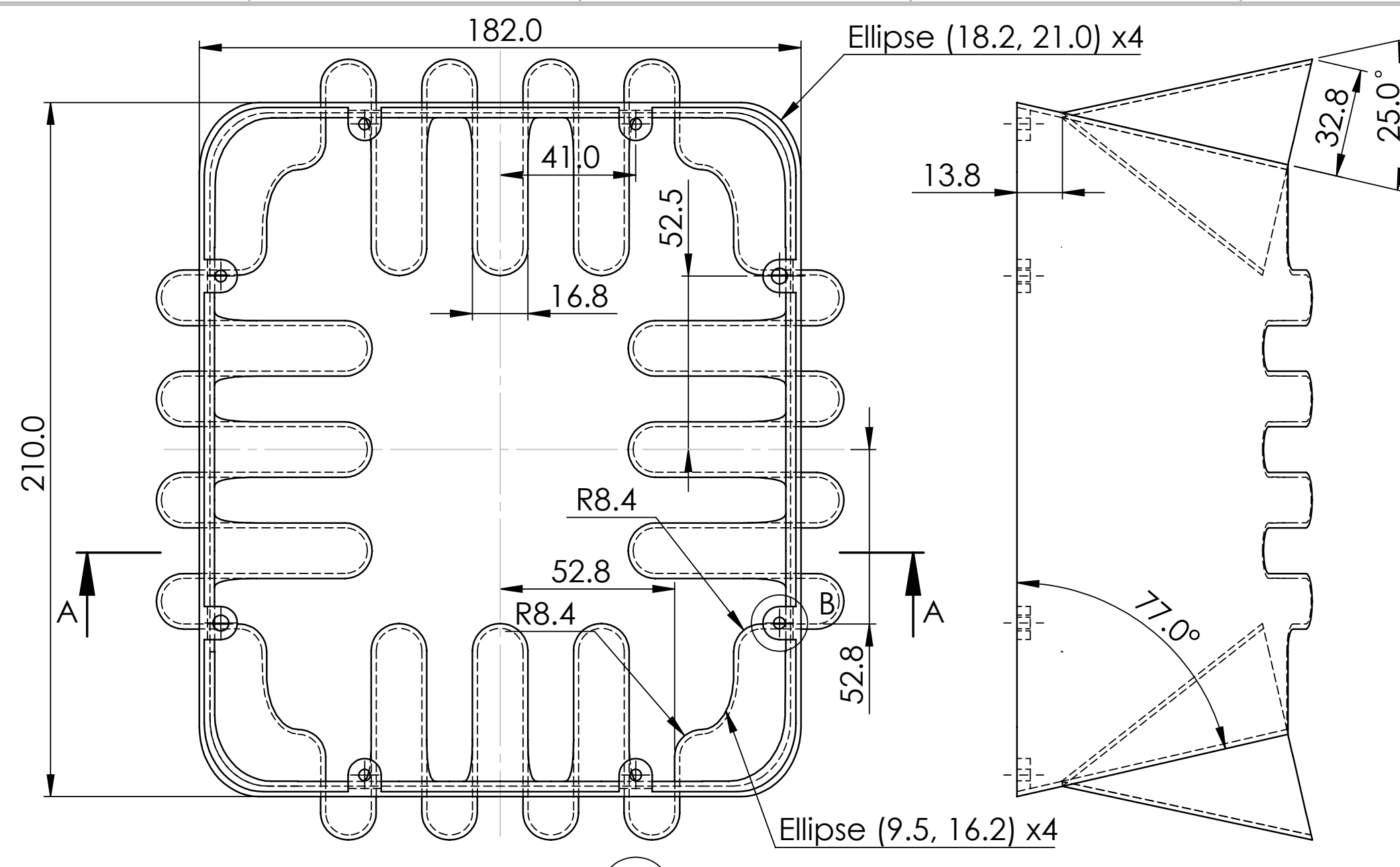

B

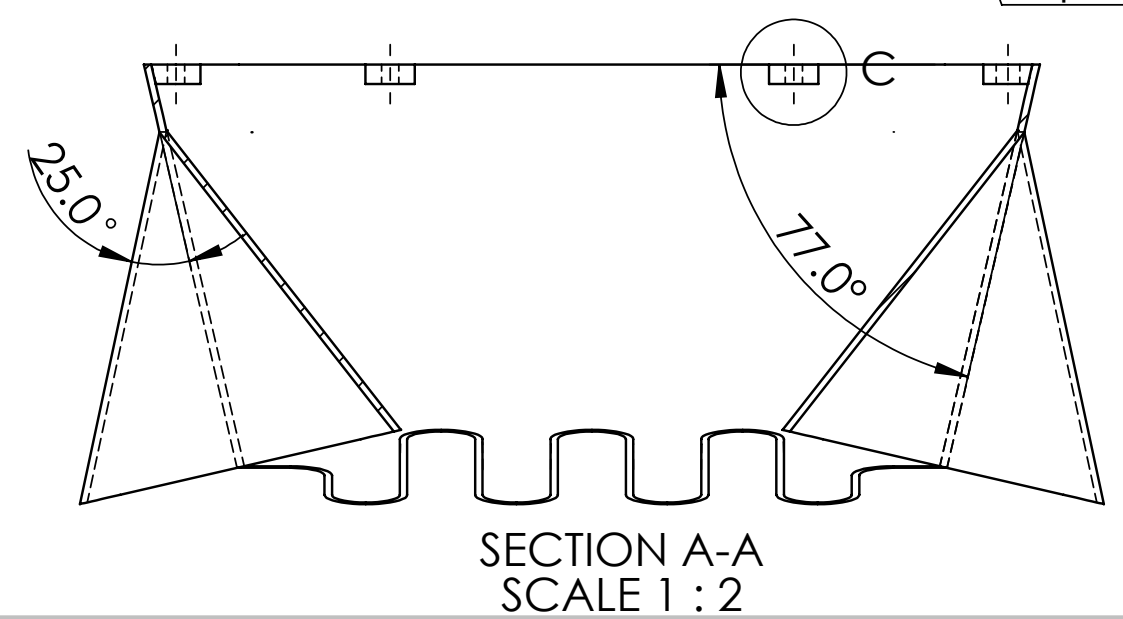

A SCALE 1:2

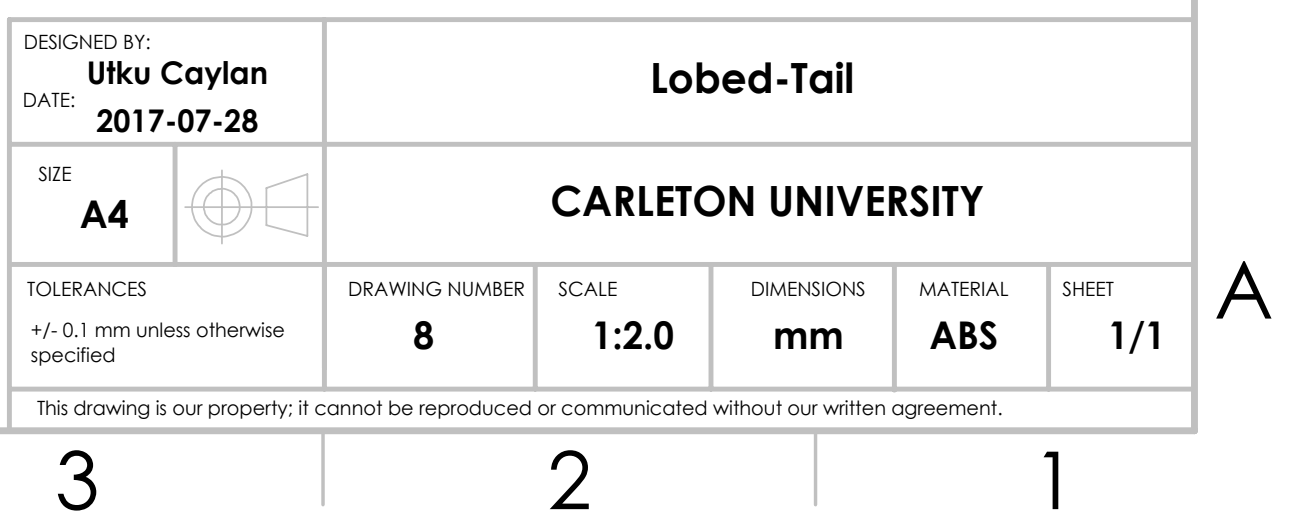

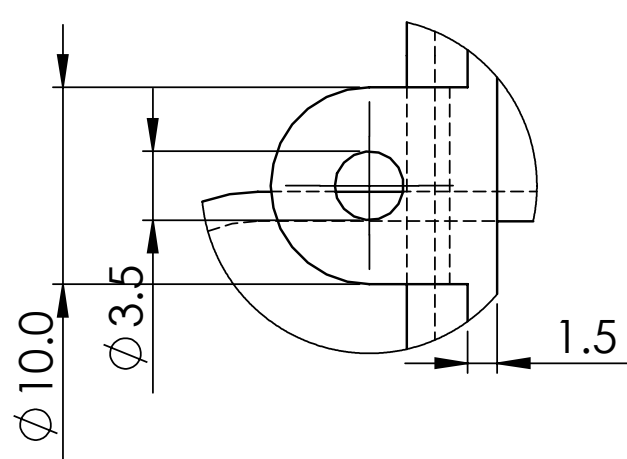

DETAIL B

SCALE $2: 1$

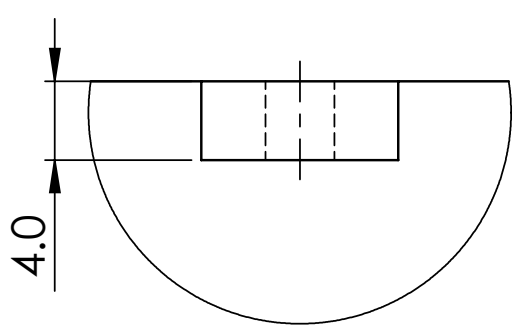

DETAIL C

D

C

B 

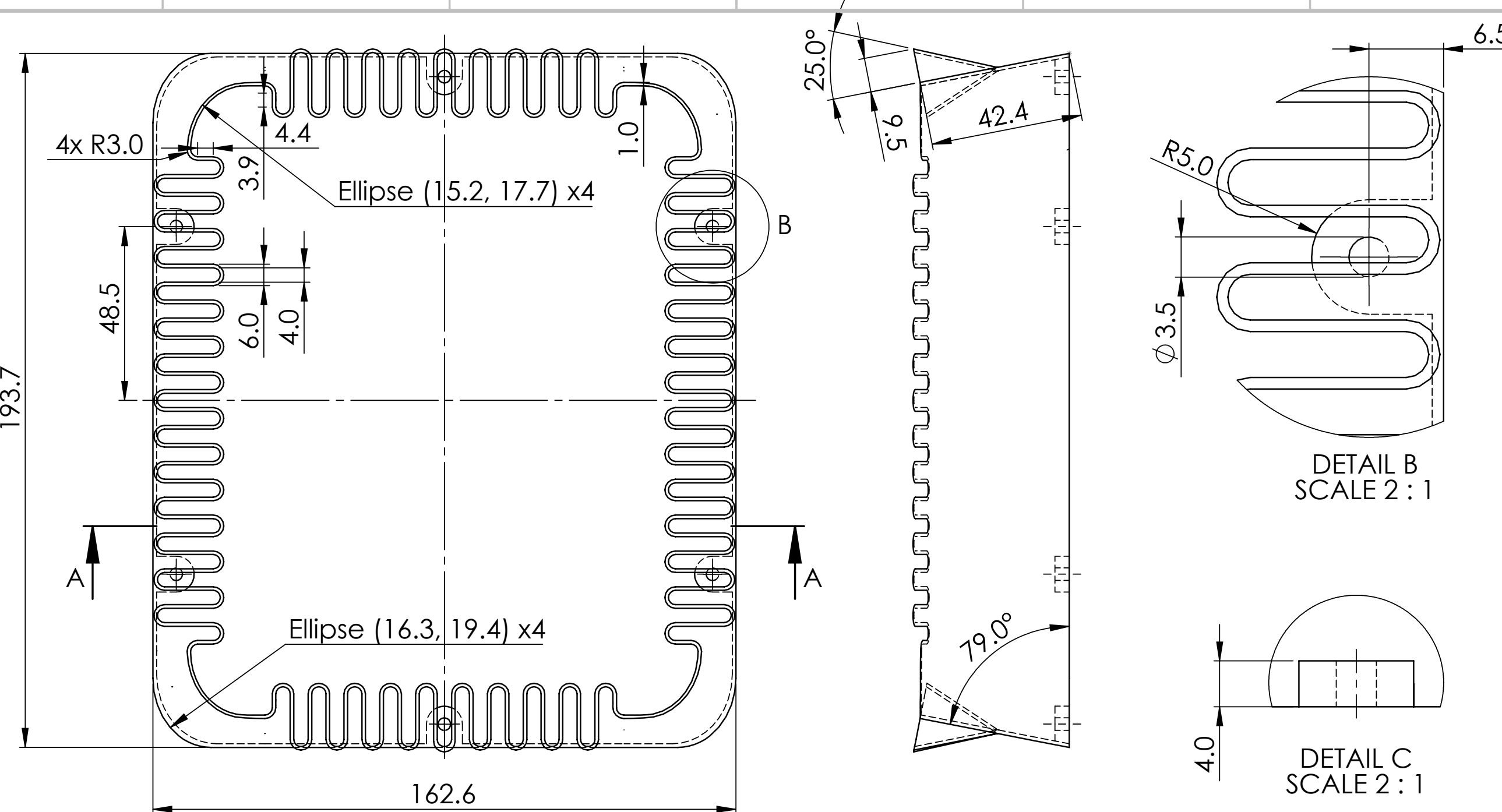

D

C SCALE $2: 1$

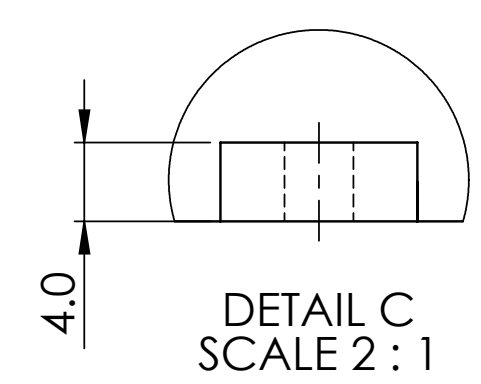

B

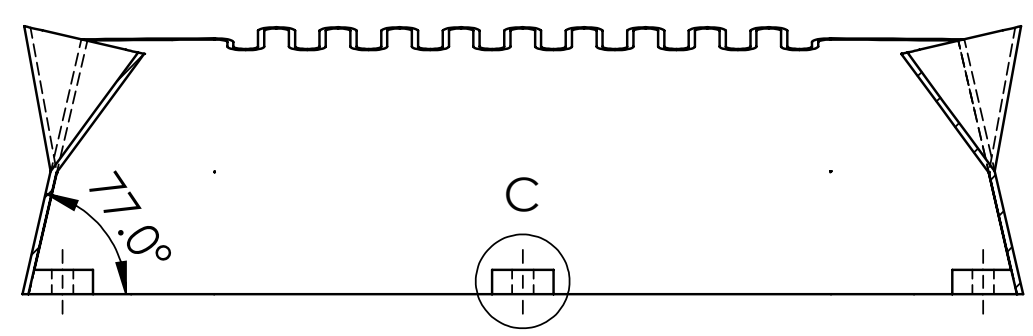

SECTION A-A SCALE $1: 1.6$

\begin{tabular}{|c|c|c|c|c|c|c|}
\hline \multicolumn{2}{|c|}{\begin{tabular}{|l} 
DESIGNED BY: \\
Utku Caylan \\
DATE: \\
$2017-07-28$ \\
\end{tabular}} & \multirow{2}{*}{\multicolumn{5}{|c|}{$\begin{array}{l}\text { Small-Lobed Boat-Tail Extension } \\
\text { CARLETON UNIVERSITY }\end{array}$}} \\
\hline A4 & $0 \square$ & & & & & \\
\hline \multicolumn{2}{|c|}{$\begin{array}{l}\text { TOLERANCES } \\
+>-1 \mathrm{~mm} \text { Unless otherwise } \\
\text { speecified }\end{array}$} & $\begin{array}{l}\text { DRAWING NUMBER } \\
9\end{array}$ & $\begin{array}{l}\text { SCALE } \\
1: 1.6\end{array}$ & $\begin{array}{l}\text { DIMENSIONS } \\
\mathbf{m m}\end{array}$ & $\begin{array}{c}\text { MATERAL } \\
\text { ABS }\end{array}$ & $\begin{array}{l}\text { SHEET } \\
1 / 1\end{array}$ \\
\hline \multicolumn{7}{|c|}{ This drawing is our property it cannot be reproduced or communicated without our wititen agreement. } \\
\hline \multicolumn{2}{|l|}{3} & & 2 & & & \\
\hline
\end{tabular}




\section{Appendix F}

\section{Structural Analysis of Bluff-Body Model}

A linear static structural analysis was performed to investigate the stress concentrations and stress distribution in the vicinity of the mounting tabs placed on the bluff-body model. ANSYS Static Structural (Version 17), a structural-analysis software package was used to carry out the finite element analysis (FEA). The installation of the bluff-body model inside the slotted-wall test section (Figure 4.4) and the mounting tabs placed on the model are illustrated in Figure F.1.

a) Side view of model installation

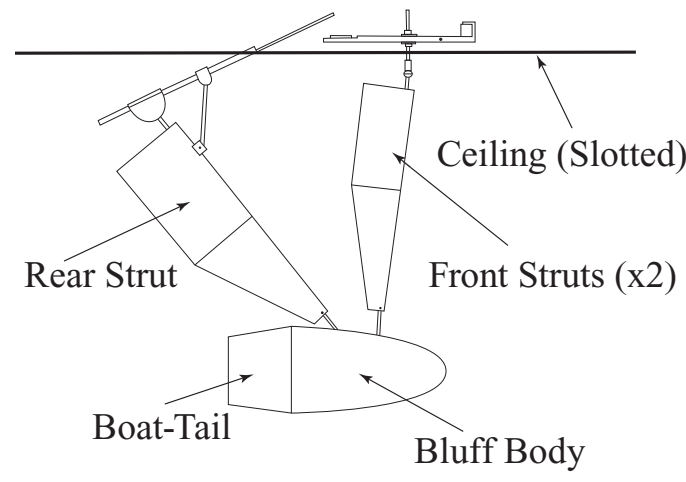

Floor (Slotted)

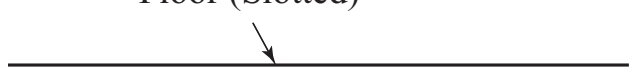

b) Mounting tabs on the bluff-body model

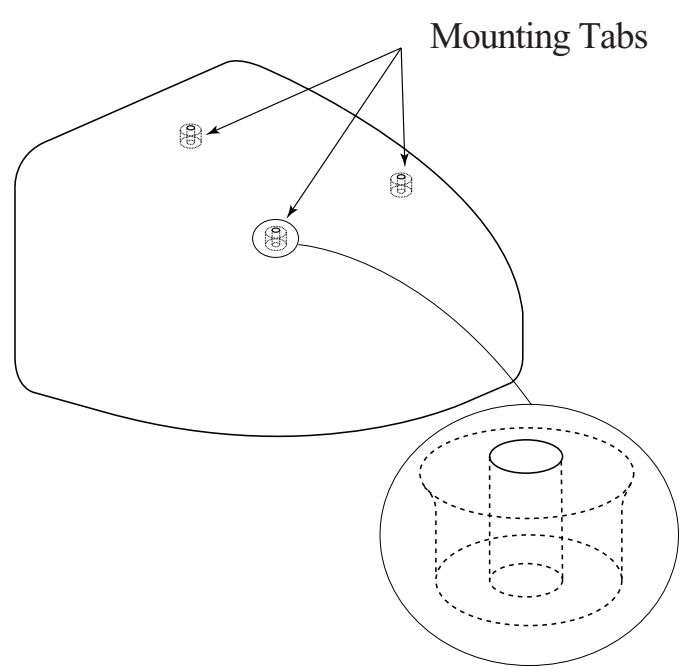

Figure F.1: Schematic of model installation and mounting tabs

\section{F.1 Computational Model}

\section{F.1.1 Computational Domain and Boundary Conditions}

The model is attached to the support struts using three mounting taps, thus the highest stress concentration is expected to occur in the vicinity of the mounting taps 


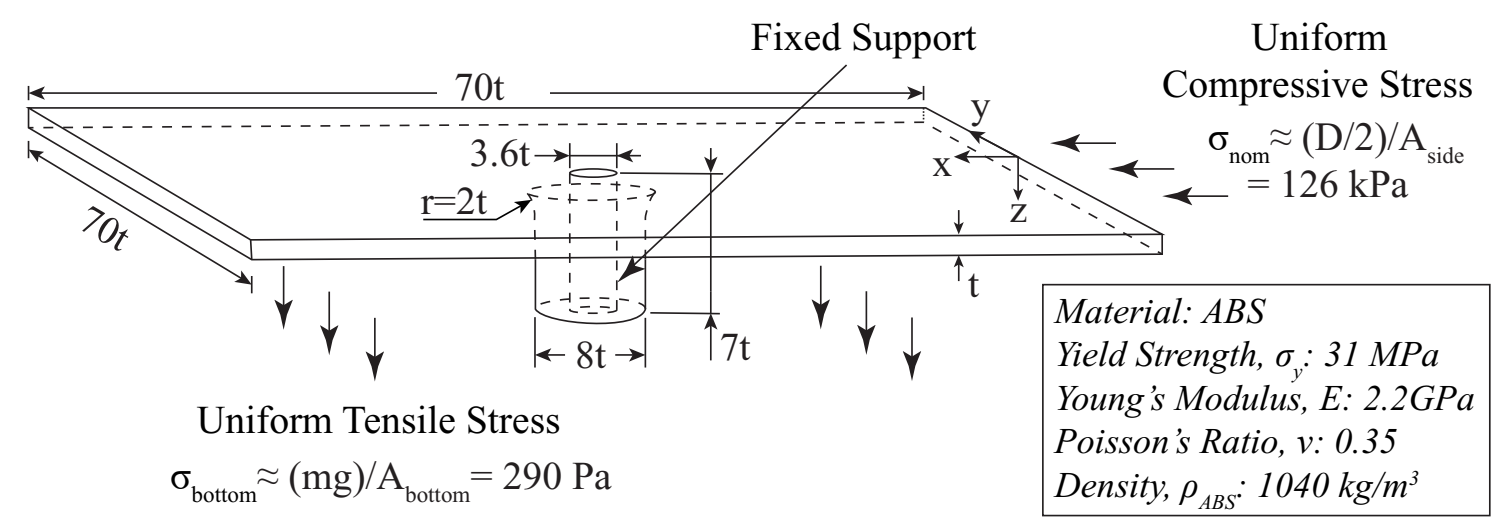

Figure F.2: Schematic of computational domain and boundary conditions

due to concentrated support loadings and geometrical discontinuities. The structural analysis is performed with a reduced computational domain that is a square flat plate with a single identical mounting tap placed on the plate center. The computational domain and boundary conditions are shown in Figure F.2. The plate thickness is set to that of the bluff-body model while each side of the plate is sized to be equal to half the model height (refer to Figure 4.1). To be sufficiently conservative prior to wind-tunnel experiments, the tab is modelled to hold half of the aerodynamic drag and full weight of the model. The aerodynamic drag, $D$, is calculated using:

$$
D=C_{D} \frac{1}{2} \rho U^{2} A
$$

where $C_{D}$ is the drag coefficient, $\rho$ is the air density, $U$ is the undisturbed upstream velocity, and $A$ is the model's projected frontal area. The drag coefficient of the bluffbody model corresponding to a flow Reynolds number based on maximum achievable wind-tunnel velocity was conservatively taken as 0.4 based on the published drag coefficient values for similar streamlined bluff bodies [170].

\section{F.1.2 Spatial Grid}

The grid is of the unstructured type and deemed most suitable for mapping a good-quality spatial grid onto the present computational domain. Table F.1 provides a summary of the relevant parameters of the spatial grid. The aspect ratio and 


\begin{tabular}{cc}
\multicolumn{2}{c}{ Table F.1: Grid specifications } \\
\hline Grid cell type & Tetrahedral \\
Max aspect ratio & 4 \\
Skewness range & $0-0.5$ \\
Max rate of change of cell size & $15 \%$ \\
Number of grid cells & 120000 \\
\hline
\end{tabular}

skewness of the grid cells should be kept within certain bounds to prevent excessive build-up of round-off errors that may hinder the rate of convergence of the iterative solution algorithm. The rate of change of the sizes of the grid cells should be kept low to prevent excessive truncation errors in the discretized spatial derivatives of the transport equations governing the flow field since truncation error determines the order of accuracy of a solution and is minimum in a uniform grid. The ranges given for these parameters in the table are consistent with the best practices in published literature [141,142]. The cell count of the grid was arrived at through a grid sensitivity analysis, with the computed stress field deemed grid independent when the change in the local stress magnitude anywhere in the domain remained below $1 \%$ upon doubling of the grid cell count in each of the three coordinate directions simultaneously.

\section{F.2 Computational Method}

\section{F.2.1 Governing Equations}

The governing equations for an isotropic linear elastic body that is in equilibrium in a three-dimensional Cartesian coordinate system can be written as follows:

$$
\frac{\partial \sigma_{i j}}{\partial x_{j}}+f_{i}=0
$$

where $\sigma_{i j}$ is the stress tensor, $f_{i}$ are the components of the body force vector per unit volume, and the indices $i, j$ are used here in Einstein notation for the three Cartesian coordinates. Assuming that the elastic material undergoes a small strain deformation, a point in the deformed body is said to have displaced an amount $u_{1}(x)$ in $\mathrm{x}_{1}$-direction, $u_{2}(x)$ in $\mathrm{x}_{2}$-direction and $u_{3}(x)$ in $\mathrm{x}_{3}$-direction, which are the 
components of the displacement vector. Using these displacement values, the engineering strains, $\varepsilon_{i j}$, are found:

$$
\varepsilon_{i j}=\frac{1}{2}\left(\frac{\partial u_{i}}{\partial x_{j}}+\frac{\partial u_{j}}{\partial x_{i}}\right)
$$

Based on the Hooke's law for a homogeneous, isotropic and linearly elastic material undergoing a small deformation, the three dimensional Navier-Cauchy equations of equilibrium can be obtained in terms of the displacements using Equations F.2 and F.3:

$$
(\lambda+\mu) \frac{\partial^{2} u_{j}}{\partial x_{j} \partial x_{i}}+\mu \frac{\partial^{2} u_{i}}{\partial x_{j} \partial x_{j}}+f_{i}=0
$$

where $\lambda$ and $\mu$ are the Lame's coefficients. Equation F.4 leads to a set of algebraic equations which are then solved to obtain the displacements.

\section{F.2.2 Discretization and Solution Method}

The linearized system of governing differential equations obtained from F.4 are solved using the finite element technique. Using a preconditioned conjugate (PCG) solver, the displacements are solved at each given node based on the given loading and the constraints set on the model. The continuity condition is met by the element shape functions ensuring that the displacement solution is continuous across the elements. Based on the relative displacement of the nodes of a given element, a stress contour is determined throughout the same element, including at the element edges and its nodes.

\section{F.3 Results and Discussion}

For the present study, the shear-strain energy (von Mises) criterion was used as the yielding criterion. This approach states that the total elastic strain energy stored in an element of material can be considered as consisting of energy stored due to change in volume and energy stored due to change in shape such as distortion or shear [171]. It is proposed that the latter contribution of stored strain energy can 
provide a viable criterion for complex yield conditions. This criterion is described by:

$$
\sigma_{e}=\frac{1}{\sqrt{2}}\left[\left(\sigma_{1}^{2}-\sigma_{2}^{2}\right)+\left(\sigma_{2}^{2}-\sigma_{3}^{2}\right)+\left(\sigma_{3}^{2}-\sigma_{1}^{2}\right)\right]^{1 / 2}
$$

where $\sigma_{1}, \sigma_{2}$ and $\sigma_{3}$ are the principal stresses and $\sigma_{e}$ is the von Mises equivalent stress. The basis of the von Mises yield criterion is that when $\sigma_{e}$ reaches the yield stress, $\sigma_{Y}$, the material is deemed to have yielded.

a)

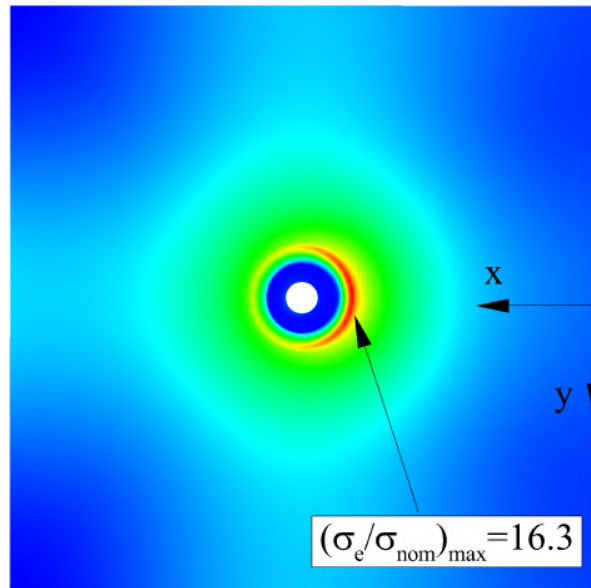

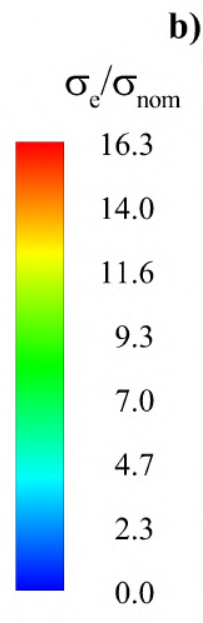

b)

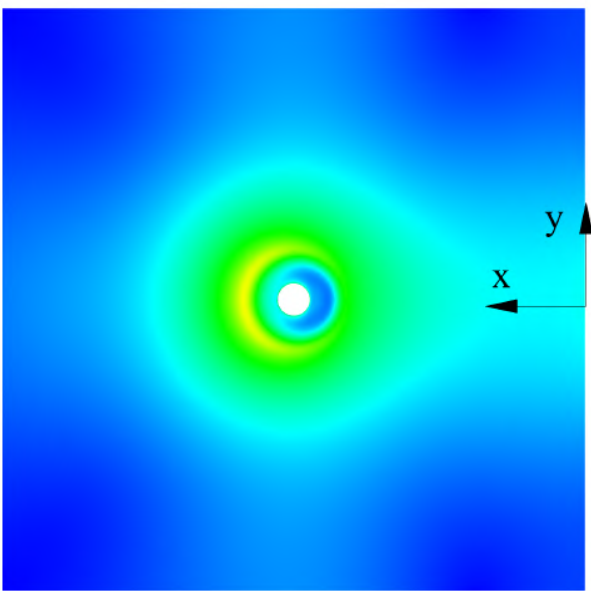

Figure F.3: $\sigma_{\mathrm{e}} / \sigma_{\text {nom }}$ contours at the a) bottom, b) top of the plate

Figure F.3 shows the normalized von Mises equivalent stress contours obtained from the structural analysis. The results indicate that the highest stress concentration occurs in the vicinity of the mounting tab at the bottom of the plate as a maximum normalized von Mises stress value of 16.3 is observed closer to the plate side where uniform compressive stress is applied. However, at the top of the plate, a relatively smaller stress concentration occurs around the opposite side of the mounting tab. The value of the highest von Mises equivalent stress that is observed at the bottom of the plate is $1.9 \mathrm{MPa}$. This stress is compared to the yield strength of acrylonitrile butadiene styrene $(\mathrm{ABS})$ plastic which the model is made of:

$$
\left(\sigma_{e}\right)_{\max }=1.9 \mathrm{MPa}<<\left(\sigma_{y}\right)_{A B S}=31 \mathrm{MPa}
$$

The comparison indicates that the mounting tab is likely to withstand the loads associated with the body and aerodynamic forces without any yielding occurring. 